Florida International University

FIU Digital Commons

FIU Electronic Theses and Dissertations

University Graduate School

3-25-2020

\title{
Equity Valuation - Seeking an Understanding through Alternative Approaches
}

\author{
Faisal Awwal \\ Florida International University, fawwa001@fiu.edu
}

Follow this and additional works at: https://digitalcommons.fiu.edu/etd

Part of the Economics Commons

\section{Recommended Citation}

Awwal, Faisal, "Equity Valuation - Seeking an Understanding through Alternative Approaches" (2020). FIU Electronic Theses and Dissertations. 4393.

https://digitalcommons.fiu.edu/etd/4393

This work is brought to you for free and open access by the University Graduate School at FIU Digital Commons. It has been accepted for inclusion in FIU Electronic Theses and Dissertations by an authorized administrator of FIU Digital Commons. For more information, please contact dcc@fiu.edu. 
FLORIDA INTERNATIONAL UNIVERSITY

Miami, Florida

EQUITY VALUATION - SEEKING AN UNDERSTANDING THROUGH ALTERNATIVE APPROACHES

A dissertation submitted in partial fulfillment of the

requirements for the degree of

DOCTOR OF PHILOSOPHY

in

ECONOMICS

by

Faisal Awwal 
To: Dean John F. Stack, Jr.

Steven J. Green School of International and Public Affairs

This dissertation, written by Faisal Awwal, and entitled Equity Valuation - Seeking an Understanding through Alternative Approaches, having been approved in respect to style and intellectual content, is referred to you for judgment.

We have read this dissertation and recommend that it be approved.

Brice Dupoyet

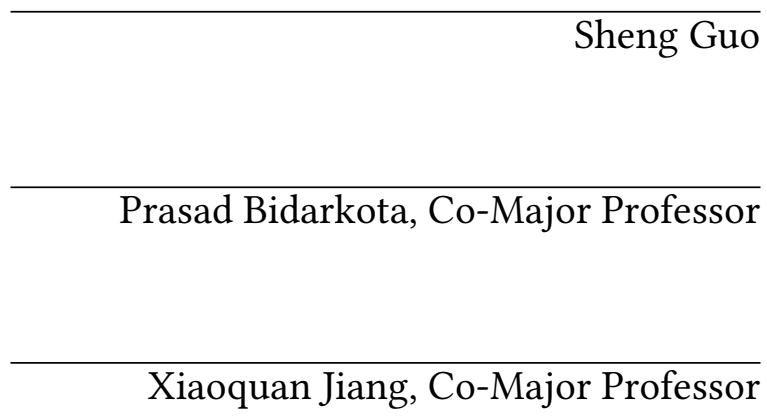

Date of Defense: March 25, 2020

The dissertation of Faisal Awwal is approved.

Dean John F. Stack, Jr. Steven J. Green School of International and Public Affairs

Andrés G. Gil

Vice President for Research and Economic Development and Dean of the University Graduate School

Florida International University, 2020 
(c) Copyright 2020 by Faisal Awwal All rights reserved. 


\section{DEDICATION}

I dedicate this dissertation to my lovely wife, Sharee, and my endearing son, Deen, for they have seen my unwavering constancy behind my laptop on the dining room table day after day tackling my research (for me, research tackles right back). Thank you for all the love, support, and periodic eye-rolling (my wife). I hope I make you both proud. I also dedicate this to my father. He put pursuit of a $\mathrm{PhD}$ in my head many years ago and I am proud to have stayed the course and come this far. 


\section{ACKNOWLEDGMENTS}

I surely would like to express my unyielding gratitude to my co-major advisors, Dr. Prasad Bidarkota and Dr. Xiaoquan Jiang for their mentorship, guidance, and sheer attention towards my research and professional efforts. I thank my dissertation committee members, Dr. Brice Dupoyet and Dr. Sheng Guo for their advisory, support, and flexibility.

Furthermore, I thank Dr. Tobias Pfutze, Dr. Mihaela Pintea, Dr. Jesse Bull, Dr. Cem Karayalcin, and all other faculty members of the Economics Department for their dedication to make the most of my $\mathrm{PhD}$ studies at FIU. I would be remiss not to express my appreciation for Mayte Rodriguez and Mariela Delgado for their assistance and advocacy over the years.

Last but furthest from least, I am completely indebted to Dr. Sonja Montas-Hunter from the Student Academic Success at FIU and Dr. Lawrence Morehouse from the Florida Education Fund. I nearly did not attend FIU due to funding constraints. These two magnificent people took a chance on me in 2016 and awarded me with a McKnight

Fellowship. I am very humbled by your generosity and advocacy to accomplish my goals. Thank you very, very much for believing in me. 


\author{
ABSTRACT OF THE DISSERTATION \\ EQUITY VALUATION - SEEKING AN UNDERSTANDING THROUGH ALTERNATIVE \\ APPROACHES \\ by \\ Faisal Awwal \\ Florida International University, 2020 \\ Miami, Florida \\ Professor Prasad Bidarkota, Co-Major Professor \\ Professor Xiaoquan Jiang, Co-Major Professor
}

This dissertation involves measuring and testing the empirical performances of equity pricing models.

The first paper extends the constant discount factor model with intrinsic bubbles developed in Froot and Obstfeld (1991) to account for autocorrelation in dividend growth rates. We derive an analytical expression for both the present value stock price and an intrinsic bubble component when dividend growth rates evolve as a Gaussian AR(1) process. Hypotheses tests favor an AR(1) process for dividend growth rates and an $\mathrm{AR}(1)$ based model developed here for price-dividends ratios over a benchmark case. Hypotheses tests also reject the absence of a bubble component in stock prices. Incorporating the bubble component into our model provides a significant improvement in fit to observed $\mathrm{P} / \mathrm{D}$ ratios and stock prices.

The second paper assesses the empirical implications of the residual income model developed in Ohlson (1995). A key assumption stipulates that next period $t+1$ residual income is a linear function of current period residual income and a latent variable referred to as 'other information'. This 'other information' is posited to contain information on next period $t+1$ residual income and reflected in current stock prices. We propose to estimate this latent 'other information' variable using a state space frame- 
work. We estimate the valuation model, within the embedded state space framework, using the Kalman filter. Performance yardsticks indicate that our state space estimation approach shows promise in valuing stocks.

The third paper attempts to estimate and study the role of 'other information' $\nu_{t}$, as theorized in the Ohlson (1995), for tracking and predicting future returns of the S\&P 500. $\nu_{t}$ is unobserved and is defined as a summary of value-relevant information about future profitability. This suggests a potential to predict subsequent returns. We apply a factor augmented vector autoregression (FAVAR) to estimate $\nu_{t}$ and evaluate its predictive performance. The FAVAR model enables us to estimate unobserved factors that are broadly captured by big data. We use principal components estimation to extract the unobserved factors from a rich set of data. Our analysis shows that the estimated $\nu_{t}$ has statistically reliable power to predict future returns. 


\section{TABLE OF CONTENTS}

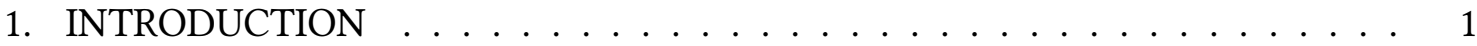

2. A STUDY OF INTRINSIC BUBBLES IN STOCK PRICES UNDER PERSISTENT DIVIDEND GROWTH RATES . . . . . . . . . . . . . . . . . . . 4

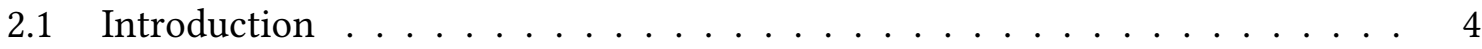

2.2 Present Value Model . . . . . . . . . . . . . . . . . . . . . . . . 7

2.3 Solution to the Model . . . . . . . . . . . . . . . . . . . 9 9

2.3.1 The Present Value Stock Price under AR(1) Dividends Growth Rate Process 9

2.3.2 Intrinsic Rational Bubbles _. . . . . . . . . . . . . . . . . . . 11

2.4 Empirical Assessment of the Model . . . . . . . . . . . . . . . . . 12

2.4 .1 Data Description . . . . . . . . . . . . . . . 12

2.4.2 Econometric Specification . . . . . . . . . . . . . . . . 13

2.4.3 Model Estimates for Dividend Growth Rates . . . . . . . . . . . . . . . . 15

2.4 .4 Present Value Stock Prices . . . . . . . . . . . . . . . . . . . 16

2.4.5 Intrinsic Bubble Parameter Estimates . . . . . . . . . . . . . . . 17

2.4.6 Price-Dividend Ratio Regression . . . . . . . . . . . . . . . . . . . . 18

2.4.7 Summary of Results and Model Comparison . . . . . . . . . . . . . . . 24

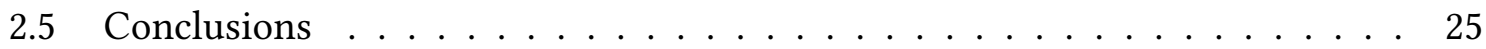

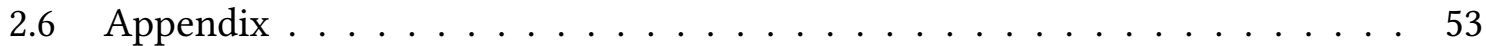

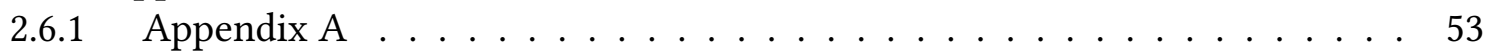

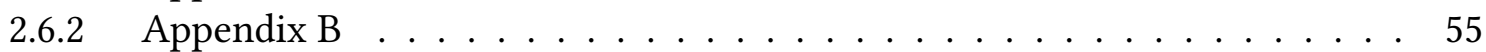

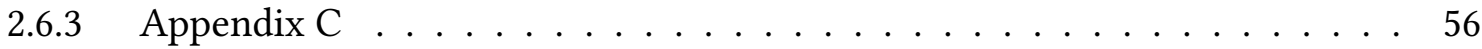

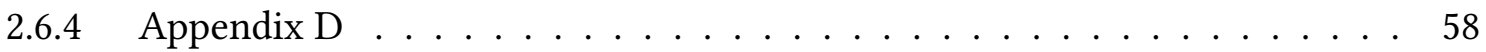

3. A STATE SPACE FRAMEWORK FOR THE RESIDUAL INCOME VALUATION MODEL OF STOCK PRICES . . . . . . . . . . . . . . . . 60

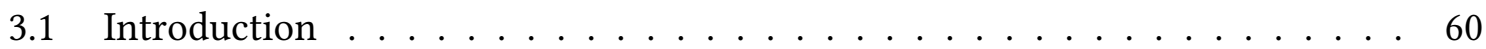

3.2 Valuation Models . . . . . . . . . . . . . . . . . 63

3.2.1 The Dividend Discount Model . . . . . . . . . . . . . . . . 63

3.2 .2 Residual Income Valuation Model . . . . . . . . . . . . . . . . . . . 64

3.2 .3 DHS Estimation Method . . . . . . . . . . . . . . . . . . 69

3.2 .4 SS Estimation Method . . . . . . . . . . . . . . . . 72

3.3 Empirical Assessment of the Model . . . . . . . . . . . . . . . . 75

3.3.1 Data Description, Aggregation, Transformation, and Cleaning Details . . . 75

3.3.2 Model Estimates . . . . . . . . . . . . . . . . 81

3.3.3 Forecasts of Stock Prices and Abnormal Earnings . . . . . . . . . . . . . . 84

3.4 Conclusions . . . . . . . . . . . . . . . . . . . 102

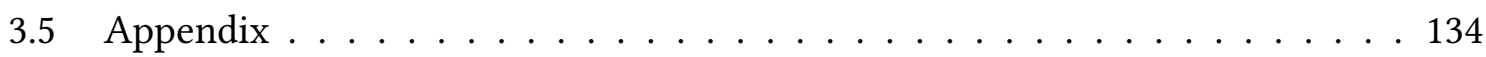

3.5.1 Alternative Representation of the State Space Model . . . . . . . . . . . . 134 
4. THE ROLE OF 'OTHER INFORMATION’ $\nu_{t}$ AS A VALUE-RELEVANT MEASURE IN TRACKING AND PREDICTING STOCK RETURNS: A FAVAR APPROACH 171

4.1 Introduction . . . . . . . . . . . . . . . . . . . 171

4.2 Residual Income Model (RIM) . . . . . . . . . . . . . . . . . . . . . . . . 173

4.2.1 Residual Income Information Dynamics and Implied Valuation Function . 173

4.2 .2 'Other information' $\nu_{t} \ldots \ldots \ldots \ldots$. . . . . . . . . . . . . . . . . . . . . . . . . 174

4.3 FAVAR Application . . . . . . . . . . . . . . . . . . . 175

4.3 .1 Framework . . . . . . . . . . . . . . . . . . . . . . 175

4.3 .2 Estimation . . . . . . . . . . . . . . . . . . . . . 177

4.3 .3 Identification Strategy $\ldots \ldots \ldots$. . . . . . . . . . . . . . 178

4.4 Empirical Assessment of the Model . . . . . . . . . . . . . . . . . . . 179

4.4 .1 Data Aggregation . . . . . . . . . . . . . . . . . . . . . . 179

4.4 .2 Empirical Results . . . . . . . . . . . . . . . . . . . . . . . 180

4.5 Conclusions . . . . . . . . . . . . . . . . . . . . . . . 187

4.6 Appendix . . . . . . . . . . . . . . . . . . . . . 196

BIBLIOGRAPHY . . . . . . . . . . . . . . . . . . . . . . . . . . . . . . . . 199

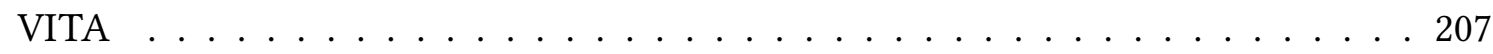




\section{LIST OF FIGURES}

FIGURE PAGE

$2.1 \quad$ Plots of Dow Jones Industrial Average Data . . . . . . . . . . . . . . 40

2.2 Plots of S\&P 500 Data . . . . . . . . . . . . . . . . 41

2.3 Probability distributions of real dividend growth rates . . . . . . . . . 42

2.4 Plots of Time-varying $\kappa$ and Constant $\kappa \ldots \ldots \ldots$. . . . . . . . 43

$2.5 \quad$ Implied AR(1) Model Results . . . . . . . . . . . . . . . . . . . 44

2.6 Implied AR(0) Model Results . . . . . . . . . . . . . . . . . . 45

2.7 Comparison of fundamental components [Implied AR(1) vs. AR(0)] . . . . 46

2.8 Comparison of both fundamental and bubble components [Implied AR(1)

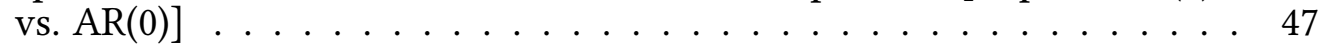

2.9 Free AR(1) Model Results . . . . . . . . . . . . . . . . . . 48

2.10 Free AR(0) Model Results . . . . . . . . . . . . . . . . . . . . . . 49

2.11 Comparison of both fundamental and bubble components [Free AR(1) vs. $\mathrm{AR}(0)] \ldots \ldots \ldots \ldots \ldots \ldots$

2.12 Comparison of both fundamental and bubble components [Implied vs. Free

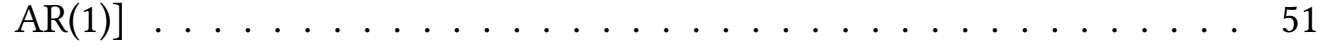

2.13 Comparison of both fundamental and bubble components [Implied vs. Free

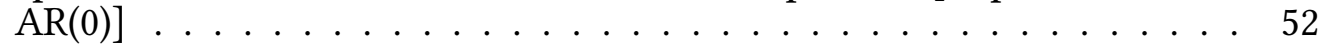

3.1 Scatter Plot of DJIA Stock Data . . . . . . . . . . . . . . . 118

3.2 Scatter Plot of S\&P 500 Stock Data . . . . . . . . . . . . . . . . 119

3.3 Comparison of In-sample RI Forecasts for 2 Stocks: SS and DHS Methods . . 120

3.4 Decomposition of In-sample RI Forecasts for 2 Stocks: $\omega x_{t}^{a} \ldots \ldots \ldots$. . . . 121

3.5 Decomposition of In-sample RI Forecasts for 2 Stocks: $\nu_{t} \ldots \ldots$. . . . . . 122

3.6 Comparison of In-sample RI Forecasts for 14 Stocks: SS and DHS Methods . 123

3.7 Comparison of In-sample Price Forecasts for 2 Stocks: SS and DHS Methods 124

3.8 Decomposition of In-sample Price Forecasts for 2 Stocks: $\alpha_{1} x_{t}^{a} \ldots \ldots$. . . 125

3.9 Decomposition of In-sample Price Forecasts for 2 Stocks: $\alpha_{2} \nu_{t} \ldots \ldots$. . . 126 
3.10 Comparison of In-sample Price Forecasts for 14 Stocks: SS and DHS Methods 127

3.11 10-year Rolling-Window Out-of-sample Price Forecasts for 2 Stocks: SS and DHS Methods . . . . . . . . . . . . . . . . . 128

3.12 Decomposition of Out-of-sample Price Forecasts for 2 Stocks: $\alpha_{1} x_{t}^{a} \ldots \ldots 129$

3.13 Decomposition of Out-of-sample Price Forecasts for 2 Stocks: $\alpha_{2} v_{t} \ldots \ldots 130$

3.14 10-year Rolling-Window Out-of-sample Price Forecasts for 14 Stocks: SS and DHS Methods . . . . . . . . . . . . . . . . . 131

3.15 Percentage Pricing Errors for 2 Stocks: SS Method . . . . . . . . . . . . . 132

3.16 Percentage Pricing Errors for 14 Stocks: SS Method . . . . . . . . . . . 133

4.1 S\&P 500 Returns and Measures of $\nu_{t} \ldots \ldots \ldots$. . . . . . . . . 194

4.2 S\&P 500 Returns and FAVAR-derived $\nu_{t} \ldots \ldots \ldots$. . . . . . . . 195 


\section{CHAPTER 1}

\section{INTRODUCTION}

"An econometrician's role is to choose among different parameters that together describe a family of possible models to best mimic measured real-world time series and to test the implications of these models.” -Nobel Laureate Lars Peter Hansen, 2014

The primary purpose of this dissertation is to explore, assess, and conjecture the dynamic behavior of U.S. equity prices. Asset pricing theory is grounded upon the concept that today's price of an asset equals its expected discounted future payoff. The keywords in that equation are 'expected' and 'discounted'. These imply two things, respectively. First, future payoffs or cash flows, in terms of distribution to asset holders, have a probability of occurrence. This probability of occurrence makes the asset inherently risky. Second, since the amount of money you have today is worth more than an identical amount in the future due to either inflation or its potential earning capacity, investors require a rate of return on these assets. This is known as the discount rate. One uses the discount rate to 'discount' the sum of future payoffs, such as dividends from a stock, which renders the present value (or fundamental value) of an asset, or, in other words, today's asset price. Together, they yield the constant discount rate present value model, which is the conventional valuation approach in asset pricing.

However, since the early 1980s, accruing evidence in the asset pricing literature has shown that changes in asset value (and asset return) in the equity markets are not consistent with the constant discount rate present value model. That is, observed stock prices persistently deviate away from their fundamental values. There are at least three possible reasons for this observation. First, changes in stock prices over time stem from changes in the discount rates, which reflect the market's time-varying risk tolerance for 
an asset. Second, using the stream of cash dividends as the standard measurement for future cash flows in the constant discount rate present value model is misspecified. This is particularly true for firms that do not issue dividends in the outset in order to reinvest the proceeds to grow its business. As such, using a present value model built upon dividends as the future payoff stream becomes impractical. Furthermore, the literature has revealed that the number of firms paying cash dividends has drastically declined since the late 1970s. In turn, the number of firms using alternative avenues of cash distribution to shareholders has considerably increased. These facts implore the need for different payoff measurements in the present value model. Third, the literature has suggested that a self-reinforcing process of price increases (decreases) can occur if investors evaluate potential gains (losses) from continuous increases (decreases) in stock prices as justification for their movement away from fundamental values. In other words, investors would be willing to pay premiums (discounts) for assets that they expect to appreciate (depreciate) in market value in the near future, regardless of their fundamental values.

This dissertation attempts to investigate the latter two possible reasons. The three papers taken together shed light on why traditional present value models do a poor job in explaining stock prices and predicting future returns, as observed in the data. This dissertation, however, does not attempt to propose which explanation, reason, or method is correct. There are countless nuances of stock market behavior, as extensively delineated throughout the three papers, that give rise to model uncertainty or misspecification. There are also countless nuances in data measurement and aggregation that naturally yield shortcomings to statistical techniques employed. Therefore, concocting an absolute conclusion, given inevitable shortcomings, would be premature and likely incorrect.

As a financial econometrician, the quote above signifies three inherent tasks. The first is to seek out alternative models introduced in the broader asset pricing literature 
that can potentially explain observed stock market behavior. The second is to assess how well the models and their respective econometric methods carried out in the literature perform empirically and reflect available stock market data. The third is to propose and implement alternative methods if there is a nontrivial deficit in the literature. Collectively, these underlie the title and theme of the dissertation. 
CHAPTER 2

\section{A STUDY OF INTRINSIC BUBBLES IN STOCK PRICES UNDER PERSISTENT DIVIDEND GROWTH RATES}

\subsection{Introduction}

Rationalization of observed stock prices is a task of great interest in financial economics. Several comprehensive surveys of the literature are available. See, for example, Hansen (2014), for a recent effort. It is well known that traditional asset pricing models do not capture variation in stock prices very well. Examples of such models include the popular constant discount factor present value model under a rational expectations framework. Deviations in stock prices from those predicted by simple present value models have proven empirically significant and persistent over time (LeRoy and Porter, 1981; Shiller, 1981). One of several approaches to rationalize these deviations in stock prices is the rational bubbles theory (Blanchard, 1979; Blanchard and Watson, 1982; Diba and Grossman, 1983, 1988a, and 1988b).

Within this framework, Froot and Obstfeld (1991) develop a specific type of rational bubble that they call a rational "intrinsic" bubble. Here, movements in stock prices are exclusively driven by economic fundamentals alone (i.e., dividends) and not from any

extraneous factors, as are common in stock price bubbles literature. Specifically, they assume a random walk process for log dividends and derive the present value stock price within a constant discount factor present value model of stock prices. Another solution to the present value model exists that violates the transversality condition. This solution for stock prices consists of the present value stock price plus a bubble component. In the intrinsic bubbles setup of Froot and Obstfeld (1991), this bubble component is driven by fundamentals alone, which are exogenous dividends. 
A random walk model of log dividends used in Froot and Obstfeld (1991) is deficient. It fails to capture observed autocorrelation in dividend growth rates. The Lintner (1956) model of corporate dividends payout assumes that firms have a target payout ratio in mind that is a fraction of current earnings. However, firms are assumed to only make partial adjustments every period. This results in a smoothing of dividends paid over time, resulting in autocorrelation in their growth rates as observed in the data. Lansing (2010) notes that, in order to generate observed persistence in price-dividend ratios, the present value-type asset pricing model requires a persistent process for dividend growth rates.

In this paper, we extend the framework of Froot and Obstfeld (1991) to account for this autocorrelation. We derive an exact analytical expression for both the present value stock price and an intrinsic bubble component when dividend growth rates evolve as a Gaussian first-order autoregressive process. Our solution for the present value stock price is an adaptation of the one provided in Burnside (1998) for the consumption-based asset pricing model under a Gaussian AR(1) process for dividend growth rates. The analytical form of the intrinsic bubble in our work is an augmented version of the one provided in Froot and Obstfeld (1991). Their framework is a special case of the one considered here.

Within a random walk framework for log dividends, Bidarkota and Dupoyet (2007) extend the intrinsic bubbles model of Froot and Obstfeld (1991) to account for observed leptokurtosis and negative skewness in dividend growth rate by modeling the innovations to the random walk as random variables drawn from a non-normal, fat-tailed probability distribution. While it would be ideal to incorporate both persistence in dividend growth rates, as they are being done here, and non-normality, as considered in Bidarkota and Dupoyet (2007), a solution to the present value model and characterization of the intrinsic bubble component poses a significant challenge under such a setting. 
Nevertheless, in the context of the present value model, at least individually, neither a fat-tailed distribution nor persistence of dividend growth rates appear to improve the fit of the present value stock prices significantly, relative to a Gaussian random walk process in the original Froot and Obstfeld (1991) paper. Though, Bidarkota and Dupoyet (2007) demonstrate that accounting for non-normal, fat tails do diminish the role of nonlinearity of the price-dividend relation, hence that of the role of bubbles in rationalizing observed stock prices.

Recent literature has studied bubbles to rationalize price movements of alternative assets and markets, such as real estate in the U.S. (Nneji et. al, 2013 and Hu and Oxley, 2018) and China (Yu, 2011), Chinese stock markets (Chang and Cai, 2016), and cryptocurrency (Cheah and Fry, 2015; Corbet et. al, 2018). Another strand of research has focused on developing new econometric methods to statistically test for the existence of speculative bubbles (Phillips et. al, 2011 and 2015; Whitehouse, 2019; Homm and Breitung, 2011; Breitung and Kruse, 2013; and Yuhn et. al, 2015).

We estimate the model developed here with two sets of annual U.S. stock price and dividends data, namely the DJIA and the S\&P 500 series, over the last century. Hypotheses tests reject an $\mathrm{AR}(0)$ process for dividend growth rates in favor of an $\mathrm{AR}(1)$ process for both data series. Likelihood ratio tests also favor the AR(1)-based model developed here for price-dividends ratios over the AR(0)-based model considered in Froot and Obstfeld (1991). Hypotheses tests also reject the absence of a bubble component in both series. This inference is robust to whether or not the parameters governing the intrinsic bubbles process are restricted to values implied by our model or freely estimated. Incorporating the bubble component into our model provides a significant improvement in fit to observed $\mathrm{P} / \mathrm{D}$ ratios and stock prices as compared to the present value stock prices alone. Lansing (2010) has completed work similar to our analytical solutions and approach by using calibration techniques to match the moments of price-dividend data, 
while we instead use econometric methods. Nonetheless, his work ignores serial correlation in dividend growth rates that we contend is a critical property observed in stock data.

We organize this paper as the following. In Section 2.2, we introduce the present value model for stock prices in which we describe the fundamental value stock price and a bubble solution that violates the transversality condition. In Section 2.3, we derive closed-form solutions to the model with a fundamental stock price component and an intrinsic bubbles component under the assumption that dividends growth evolves as an $\mathrm{AR}(1)$ process. In Section 2.4, we introduce the data and econometric specifications, and provide a series of empirical results and inferences. We summarize our main findings in the Section 2.5.

\subsection{Present Value Model}

The present value model with a constant discount rate is given by:

$$
\mathrm{P}_{\mathrm{t}}=\mathrm{e}^{-\mathrm{r}} \mathrm{E}_{\mathrm{t}}\left[\mathrm{D}_{\mathrm{t}}+\mathrm{P}_{\mathrm{t}+1}\right] .
$$

Here, $P_{t}$ is the real price of a share at the beginning of period $\mathrm{t}, D_{t}$ is the real dividend per share paid out over period $\mathrm{t}, \mathrm{r}$ is the non-stochastic and constant discount rate, $E_{t}$ is the mathematical expectation conditioned on information available at the start of period $t$. It is often useful to think about this pricing equation as arising from a Lucas (1978)-type asset pricing model under risk neutrality. 
On forward iteration, the present value equation yields:

$$
P_{t}=\sum_{s=t}^{\infty} \mathrm{e}^{-\mathrm{r}(\mathrm{s}-\mathrm{t}+1)} \mathrm{E}_{\mathrm{t}}\left(\mathrm{D}_{\mathrm{s}}\right)+\lim _{s \rightarrow \infty} \mathrm{e}^{-\mathrm{rs}} \mathrm{E}_{\mathrm{t}}\left(\mathrm{P}_{\mathrm{s}}\right)
$$

One solution to stock prices in the above equation, denoted $P_{t}^{p v}$ is obtained by imposing the transversality condition:

$$
\lim _{s \rightarrow \infty} \mathrm{e}^{-\mathrm{rs}} \mathrm{E}_{\mathrm{t}}\left(\mathrm{P}_{\mathrm{s}}\right)=0
$$

Imposing the transversality condition on Equation (2.2) gives:

$$
\mathrm{P}_{\mathrm{t}}^{\mathrm{pv}}=\sum_{s=t}^{\infty} \mathrm{e}^{-\mathrm{r}(\mathrm{s}-\mathrm{t}+1)} \mathrm{E}_{\mathrm{t}}\left(\mathrm{D}_{\mathrm{s}}\right)
$$

Thus, this equation provides the fundamental value of the stock price. One specifies an exogenous stochastic process for dividends and evaluates $P_{t}^{p v}$.

There exist other solutions to the present value model given in Equation (2.1) that do not satisfy the transversality condition in Equation (2.3). For instance, let $\left\{\mathrm{B}_{\mathrm{t}}\right\}_{\mathrm{t}=0}^{\infty}$ be any sequence of random variables that satisfy:

$$
\mathrm{B}_{\mathrm{t}}=\mathrm{e}^{-\mathrm{r}} \mathrm{E}_{\mathrm{t}}\left\{\mathrm{B}_{\mathrm{t}+1}\right\}
$$

One can easily show that $\left(P_{t}^{\mathrm{pv}}+\mathrm{B}_{\mathrm{t}}\right)$ satisfies Equation (2.1) but violates Equation (2.3) for all $B_{t} \neq 0$. 
If $B_{t}$ is constructed as a function of the fundamentals alone, i.e., as a function of the dividends $D_{t}$ alone in the present value model of Equation (2.1), it is termed an intrinsic rational bubble by Froot and Obstfeld (1991). Intrinsic bubbles turn out to be a non-linear function of dividends. Their exact functional form depends on the assumed stochastic process for the dividends.

\subsection{Solution to the Model}

In this section, we obtain an exact analytical solution for the present value stock price $P_{t}^{p v}$ when the dividend growth rate follows a first-order autoregressive process. We also derive conditions under which a posited functional form for $B_{t}$ satisfies all the conditions for a rational intrinsic bubble.

\subsubsection{The Present Value Stock Price under AR(1) Dividends Growth}

\section{Rate Process}

Let $\mathrm{x}_{\mathrm{t}} \equiv \ln \left(\mathrm{D}_{\mathrm{t}}\right)-\ln \left(\mathrm{D}_{\mathrm{t}-1}\right)$ denote the dividend growth rate. We assume that $x_{t}$ stochastically evolves as a first-order autoregressive process:

$$
\mathrm{x}_{\mathrm{t}}-\mu=\rho\left(\mathrm{x}_{\mathrm{t}-1}-\mu\right)+\xi_{\mathrm{t}}, \quad|\rho|<1, \quad \xi_{\mathrm{t}} \sim \operatorname{iid~N}\left(0, \sigma^{2}\right)
$$

One can now derive the present value stock price by evaluating the right hand side of Equation (2.4). Following up on the results in Burnside (1998), Appendix A shows that the present value stock price is given by: 


$$
\mathrm{P}_{\mathrm{t}}^{\mathrm{pv}}=\mathrm{D}_{\mathrm{t}} \sum_{\mathrm{s}=\mathrm{t}}^{\infty} \exp \left\{-\mathrm{r}(\mathrm{s}-\mathrm{t}+1)+\mathrm{b}_{\mathrm{s}-\mathrm{t}}\left(\mathrm{x}_{\mathrm{t}}-\mu\right)+\mathrm{a}_{\mathrm{s}-\mathrm{t}}\right\}
$$

where

$$
a_{s-t}=(s-t) \mu+\frac{\sigma^{2}}{2(1-\rho)^{2}}\left[(s-t)-2 \frac{\rho}{1-\rho}\left(1-\rho^{s-t}\right)+\rho^{2} \frac{1-\rho^{2(s-t)}}{1-\rho^{2}}\right]
$$

and

$$
\mathrm{b}_{\mathrm{s}-\mathrm{t}}=\frac{\rho}{1-\rho}\left\{1-\rho^{\mathrm{s}-\mathrm{t}}\right\}
$$

The following theorem provides conditions for the infinite summation in Equation (2.7) to converge, and hence for the priceâĂŞdividend ratio to be finite.

Theorem 1. The series in Equation (2.7) converges if

$$
\mathrm{R} \equiv \exp \left\{-\mathrm{r}+\mu+\frac{\sigma^{2}}{2\left(1-\rho^{2}\right)}\right\}<1
$$

Proof: See Appendix B.

The next theorem derives an expression for the mean of the fundamental stock pricedividend ratio, i.e., the unconditional expectation of $\mathrm{P}_{t}^{\mathrm{pv}} / \mathrm{D}_{\mathrm{t}}$. It also provides conditions under which this mean is finite.

Theorem 2: The mean of the price dividend ratio $\mathrm{P}_{t}^{\mathrm{pv}} / \mathrm{D}_{\mathrm{t}}$ is given by:

$$
\mathrm{E}\left(\mathrm{P}_{\mathrm{t}}^{\mathrm{pv}} / \mathrm{D}_{\mathrm{t}}\right)=\sum_{\mathrm{i}=0}^{\infty} \exp \left\{-\mathrm{r}(\mathrm{i}+1)+\mathrm{a}_{\mathrm{i}}+\frac{\mathrm{b}_{\mathrm{i}}^{2} \sigma^{2}}{2\left(1-\rho^{2}\right)}\right\}
$$


where

$$
a_{i}=i \mu+\frac{\sigma^{2}}{2(1-\rho)^{2}}\left[i-2 \frac{\rho}{1-\rho}\left(1-\rho^{i}\right)+\rho^{2} \frac{1-\rho^{2 i}}{1-\rho^{2}}\right]
$$

and

$$
\mathrm{b}_{\mathrm{i}}=\frac{\rho}{1-\rho}\left\{1-\rho^{\mathrm{i}}\right\}
$$

It is finite if $\mathrm{R} \equiv \exp \left\{-\mathrm{r}+\mu+\frac{\sigma^{2}}{2\left(1-\rho^{2}\right)}\right\}<1$.

Proof: See Appendix C

\subsubsection{Intrinsic Rational Bubbles}

Let us postulate that intrinsic rational bubbles take the form:

$$
\mathrm{B}\left(\mathrm{D}_{\mathrm{t}}\right)=\mathrm{cD}_{\mathrm{t}}^{\lambda} \exp \left\{\mathrm{hx}_{\mathrm{t}}\right\}
$$

Here, $\lambda>0$ for the bubble to grow with an increase in dividends, $c>0$ to ensure nonnegativity of stock prices, and $\mathrm{h}$ is a constant.

Appendix D shows that the functional form for the intrinsic bubble in Equation (2.14) satisfies Equation (2.5) defining a bubble, provided that $\lambda$ and $\mathrm{h}$ are chosen to satisfy:

$$
\mathrm{r}=(\lambda+\mathrm{h})(1-\rho) \mu+(\lambda+\mathrm{h})^{2} \sigma^{2} / 2
$$

and

$$
\mathrm{h}=(\lambda+\mathrm{h}) \rho .
$$


If the dividend growth rate stream follows an $\mathrm{AR}(0)$ process, then the solution for the present value stock price is easily obtained by setting $\rho=0$ in the equations above. One can readily show that the expression obtained for the present value stock price in this case is identical to the one given in Froot and Obstfeld (1991). From Equation (2.16) h = 0 when $\rho=0$. Therefore, the bubble component of the stock price given in Equation (2.14) reduces to $B\left(D_{t}\right)=c D_{t}^{\lambda}$, exactly the expression in Froot and Obstfeld (1991). In this case the conditions needed for convergence of the fundamental stock price-dividend ratio as well as the conditions for $B\left(D_{t}\right)$ to be a rational intrinsic bubble are also identical to those in Froot and Obstfeld (1991).

\subsection{Empirical Assessment of the Model}

\subsubsection{Data Description}

For empirical assessment of the model, we employ two aggregate stock price indices: the Dow Jones Industrial Average (DJIA) and the S\&P 500. We retrieved annual DJIA index data, including average, yearly closing values and dividends for a sample period of 1920-2017 from two data sources. For the 1920-2005 period, we use the 2006 Value Line publication, A Long-Term Perspective: Dow Jones Industrial Average, 1920âĂŞ2005 and for the 2006-2017 period, we use Standard \& PoorâĂŹs Compustat database. Additionally, we retrieved monthly S\&P 500 index data, including closing values and dividends for a sample period of 1900-2018 from Robert ShillerâĂŹs Irrational Exuberance (2000) publication. Each series used in the Shiller dataset is of January values. Although S\&P 500 data spanning from 1871 are available, to follow Froot and Obstfeld (1991), we begin the series in 1900 . 
Table 2.1 provides summary statistics on real dividend growth rates and $\mathrm{P} / \mathrm{D}$ ratios for both stock indices. As established in extant literature, dividend growth rates and P/D ratios both indicate strong and statistically significant leptokurtosis, negative skewness for the former and positive for the latter, with normality being strongly rejected for both series. The two series also exhibit strong first-order autocorrelation. Figures 2.1 and 2.2 plot real stock prices, real dividends and their growth rates, and price-dividend ratios for DJIA and S\&P 500, respectively. Our objective in this paper is to attempt to rationalize movements in price-dividend ratios, and hence movements in the stock prices themselves, through movements in dividend growth rates which we take here to be exogenous.

\subsubsection{Econometric Specification}

Empirical evaluation of our model requires specification of an exogenous stochastic process for dividend growth rates. This is taken to be the AR(1) process given in Equation (2.6):

$$
\mathrm{x}_{\mathrm{t}}-\mu=\rho\left(\mathrm{x}_{\mathrm{t}-1}-\mu\right)+\xi_{\mathrm{t}}, \quad|\rho|<1, \quad \xi_{\mathrm{t}} \sim \operatorname{iid~} \mathrm{N}\left(0, \sigma_{\xi}^{2}\right)
$$

Assumption of a normal distribution for $\xi_{t}$ is inconsistent with its strong rejection reported in Table 2.1 and discussed in Section 2.4.1. A non-normal, fat-tailed probability distribution that explicitly accounts for leptokurtosis and negative skewness in the dividend growth rate was considered in Bidarkota and Dupoyet (2007). However, their analysis ignored persistence in dividend growth rates and considered a random walk process, instead. While it would be ideal to incorporate both persistence in dividend growth rates, as they are being done here, and non-normality, as considered in Bidarkota and Dupoyet (2007), a solution to the present value model and characterization of the intrinsic bubble component pose a significant challenge under such a setting. Burnside 
(1998) provides a solution to the consumption-based asset pricing model under a Gaussian $\mathrm{AR}(1)$ process for dividend growth rates. However, he neither considers intrinsic bubbles in his work nor undertakes an empirical assessment of the model.

Considering the discussion following Equation (2.5), one can write the complete solution to the present value model as:

$$
\mathrm{P}_{\mathrm{t}}=\mathrm{P}_{\mathrm{t}}^{\mathrm{pv}}+\mathrm{B}_{\mathrm{t}}
$$

Now using Equations (2.7)-(2.9) and (2.14)-(2.16), one obtains:

$$
\mathrm{P}_{\mathrm{t}}=\kappa_{\mathrm{t}} \mathrm{D}_{\mathrm{t}}+\mathrm{cD}_{\mathrm{t}}{ }^{\lambda} \exp \left\{\mathrm{hx}_{\mathrm{t}}\right\}
$$

Here

$$
\kappa_{\mathrm{t}}=\sum_{\mathrm{s}=\mathrm{t}}^{\infty} \exp \left\{-\mathrm{r}(\mathrm{s}-\mathrm{t}+1)+\mathrm{b}_{\mathrm{s}-\mathrm{t}}\left(\mathrm{x}_{\mathrm{t}}-\mu\right)+\mathrm{a}_{\mathrm{s}-\mathrm{t}}\right\}
$$

from Equation (2.7), and $a_{s-t}$ and $b_{s-t}$ are given in Equations (2.8) and (2.9), respectively. Dividing Equation (2.19) by $D_{t}$, we can write:

$$
\frac{\mathrm{P}_{\mathrm{t}}}{\mathrm{D}_{\mathrm{t}}}=\kappa_{\mathrm{t}}+\mathrm{cD}_{\mathrm{t}}^{\lambda-1} \exp \left\{\mathrm{hx}_{\mathrm{t}}\right\}
$$

We follow standard practice in the literature by augmenting the stock price-dividend ratio obtained by solving the present value equation with a regression residual $\eta_{t}$ when fitting the model to the data. As noted by Hamilton (1986), the regression residual captures omitted variables such as time-varying real interest rates, risk premia, and changes in tax laws. 
Consequently, one obtains the following econometric model for the stock pricedividend ratio:

$$
\frac{\mathrm{P}_{\mathrm{t}}}{\mathrm{D}_{\mathrm{t}}}=\mathrm{b}_{0} \kappa_{\mathrm{t}}+\mathrm{b}_{1} \mathrm{D}_{\mathrm{t}}^{\lambda-1} \exp \left\{\mathrm{hx}_{\mathrm{t}}\right\}+\eta_{\mathrm{t}}, \quad \eta_{\mathrm{t}} \sim \text { iid } \mathrm{N}\left(0, \sigma_{\eta}^{2}\right)
$$

where $b_{0}, b_{1}, \lambda$, and $h>0$. The error term $\eta_{t}$ is assumed to be independent of the innovations $\xi_{t}$ to the dividend growth rate in Equation (2.17), at all leads and lags. $\kappa_{t}$ can be thought of as a time-varying dividends multiplier.

Thus, our econometric specification, motivated by the present value model, is made up of Equations (2.17) and (2.22), for the dividend growth rates and price-dividend ratios respectively, subject to restrictions on the parameters governing the intrinsic bubble process given in Equations (2.15) and (2.16). These restrictions can now be stated as follows:

$$
\mathrm{r}=(\lambda+\mathrm{h})(1-\rho) \mu+(\lambda+\mathrm{h})^{2} \sigma_{\xi}^{2} / 2
$$

and $\mathrm{h}=(\lambda+\mathrm{h}) \rho$.

\subsubsection{Model Estimates for Dividend Growth Rates}

Table 2.2 reports maximum likelihood estimates of the AR(1) model for dividend growth rates given in Equation (2.17) in the top panel. The estimates for $\mu, \sigma^{2}$, and $\rho$ are close to the empirical mean, variance, and first-order autocorrelation coefficient of raw dividend growth rates reported in Table 2.1. Froot and Obstfeld (1991) consider a random walk process for dividends, i.e. an $\mathrm{AR}(0)$ process for their growth rates $x_{t}$ :

$$
\mathrm{x}_{\mathrm{t}}=\mu+v_{\mathrm{t}}, \quad v_{\mathrm{t}} \sim \operatorname{iid} \mathrm{N}\left(0, \sigma_{v}^{2}\right)
$$


Estimates of the benchmark $\mathrm{AR}(0)$ process for dividend growth rate are reported in Panel B of Table 2.2.

A test of the benchmark $\operatorname{AR}(0)$ versus $A R(1)$ process for dividend growth rates can be conducted by testing for $\rho=0$. The likelihood ratio (LR) test for such a hypothesis is reported in the last column of Table 2.2. The test rejects $\operatorname{AR}(0)$ for both series at better than the 10 percent significance level. This provides empirical justification for considering extension of the work reported in Froot and Obstfeld (1991).

Figure 2.3 plots the unconditional distributions of the $\mathrm{AR}(1)$ and $\mathrm{AR}(0)$ models, along with the kernel density of the dividend growth rates. Figure 3a suggests that the $\operatorname{AR}(0)$ model renders a better fit to the DJIA kernel density, while Figure $3 \mathrm{~b}$ indicates that the AR(1) model provides a better fit for the S\&P 500 kernel density. However, neither appear to provide a significant improvement in fit over the other. This is not surprising, given that the maximum likelihood estimates for $\mu$ and $\sigma^{2}$ for both models are close to the empirical mean and variance of raw dividend growth rates reported in Table 2.1.

\subsubsection{Present Value Stock Prices}

In order to calculate present value stock prices implied by our model, we need a value for the constant discount rate, r. As in Froot and Obstfeld (1991) and and Dupoyet (2007), we choose r equal to 8.6\%. Using maximum likelihood parameter estimates from Table 2.2, we verify that the convergence condition given in Equation (2.10), required for finiteness of the present value stock price given in Equation (2.7), is satisfied. The time-varying present value stock price to dividends ratio, or the dividends multiplier, $\kappa_{t}$ is estimated by evaluating the expression on the right hand side of Equation (2.20), with appropriate truncation. Figure 2.4 plots these values for the two data series. Their mean values are reported in the last column of the top panel in Table 2.3. We note 
that these values are considerably below their empirical counterparts reported in Table 2.1. However, these mean $\kappa_{t}$ values are larger than the constant $\kappa$ estimates of about 14 , reported in Froot and Obstfeld (1991) and Bidarkota and Dupoyet (2007) for the Gaussian $\mathrm{AR}(0)$ dividend growth rate.

\subsubsection{Intrinsic Bubble Parameter Estimates}

Implied values of the parameters $\lambda$ and $h$, governing the intrinsic bubble process given in Equation (2.14), are obtained by solving Equations (2.23) and (2.16).

For the benchmark $\mathrm{AR}(0)$ process for dividend growth rates in Equation (2.24), the form for the intrinsic bubbles term in Equation (2.14) reduces to:

$$
\mathrm{B}\left(\mathrm{D}_{\mathrm{t}}\right)=\mathrm{cD}_{\mathrm{t}}^{\lambda}
$$

with $\lambda>0$ for the bubble to grow with increasing dividends, and $c>0$ to ensure nonnegativity of stock prices, as for the $\mathrm{AR}(1)$ dividend growth rate process. The constant $\mathrm{h}$ that appears in the $\mathrm{AR}(1)$ case is now equal to zero. Parameter restrictions governing the intrinsic bubble process given in Equations (2.15) and (2.16) now reduce to:

$$
\mathrm{r}=\lambda(\mu+\lambda) \sigma_{v}^{2}
$$

Table 2.3 reports values for the intrinsic bubble parameters for both $\operatorname{AR}(1)$ and $\operatorname{AR}(0)$ process for dividend growth rates. In Panel A, for the AR(1) process, the solution yields values of 1.772 and 2.148 for $\lambda$ for the two data series. By contrast, Panel B reports values of $\lambda=2.062$ and 2.609 for the $\mathrm{AR}(0)$ process. For comparison, Froot and Obstfeld (1991) obtain an estimate of $\lambda=2.74$ while Bidarkota and Dupoyet (2007) obtain $\lambda=2.50$ for 
the Gaussian $\mathrm{AR}(0)$ process. This is not surprising given the expression for the intrinsic bubble in the $\mathrm{AR}(1)$ case given by Equation (2.14):

$$
\mathrm{B}\left(\mathrm{D}_{\mathrm{t}}\right)=\mathrm{cD} \mathrm{t}_{\mathrm{t}}^{\lambda} \exp \left\{\mathrm{hx}_{\mathrm{t}}\right\}
$$

which can be re-expressed as:

$$
\mathrm{B}\left(\mathrm{D}_{\mathrm{t}}\right)=\mathrm{cD}_{\mathrm{t}}^{(\lambda+h)} \mathrm{D}_{\mathrm{t}-1}^{(-h)}
$$

In Panel A, for the AR(1) process, the solution yields values of 0.557 and 0.73 for parameter $\mathrm{h}$ for the two data series.

\subsubsection{Price-Dividend Ratio Regression}

\section{Models}

We now proceed with estimation of the econometric model for price-dividends ratios given in Equation (2.22). We estimate several versions of this model with the two data series. The first two rows of Table 2.4 list the two main models of interest at this point. The model in Equation (2.22), along with the restrictions specified by Equations (2.16) and (2.23), is the primary model of stock prices developed in this paper, with an $\operatorname{AR}(1)$ process driving the dividend growth rate and comprising of intrinsic bubbles. This is referred to as the Implied AR(1) Model. When estimating this model, the dividends growth, $x_{t}$ follows an AR(1) process whose parameter values are the estimates reported in Panel A of Table 2.2. The values of the bubble component parameters $\lambda$ and $\mathrm{h}$ are set equal to the implied parameter values reported in Panel A of Table 2.3. Implied $\mathrm{AR}(0)$ Model is the version of the above model, where the dividend growth rates follow 
an $\mathrm{AR}(0)$ process instead, given by Equation (2.24). The econometric model for pricedividends ratio is now:

$$
\frac{\mathrm{P}_{\mathrm{t}}}{\mathrm{D}_{\mathrm{t}}}=\mathrm{b}_{0} \kappa+\mathrm{b}_{1} \mathrm{D}_{\mathrm{t}}^{\lambda-1}+v_{\mathrm{t}}, \quad v_{\mathrm{t}} \sim \operatorname{iid~} \mathrm{N}\left(0, \sigma_{v}^{2}\right)
$$

where $\kappa=\sum_{\mathrm{s}=\mathrm{t}}^{\infty} \exp \left\{-\mathrm{r}(\mathrm{s}-\mathrm{t}+1)+\mathrm{a}_{\mathrm{s}-\mathrm{t}}\right\}$, and $a_{s-t}$ is given in Equation (2.8) with $\rho=0$. This is the model estimated by Froot and Obstfeld (1991). When estimating this model, the dividends growth, $x_{t}$ follows an $\mathrm{AR}(0)$ process whose parameter values are the estimates reported in Panel B of Table 2.2. As stated in the discussion in Section 2.3.2, in this instance, $\mathrm{h}=0$. The value of the bubble component parameter $\lambda$ is now set equal to the implied parameter values reported in Panel B of Table 2.3. For each of these models, we estimate an unrestricted version which is the model described above. This is referred to as Sub-Model A. We also estimate three restricted versions, referred to as Sub-Models B, C, and D. The restrictions describing these three versions of the models are specified in the last three rows of Table 2.4. Sub-Model B is a semi-restricted model with $b_{0}=1$. Sub-Model C is a semi-restricted model with $b_{1}=0$. Sub-Model D is a restricted model with $b_{1}=0$ and $b_{0}=1$. As stated earlier, our primary model of interest is the Implied AR(1) Model developed in this paper in the present value context with an intrinsic bubble component. Implied $\operatorname{AR}(0)$ Model is the one estimated by Froot and Obstfeld (1991). Within these two models, Sub-Model A is the unrestricted version with an intrinsic bubble component whereas Sub-Model D is the most restricted version with no bubble component and the mean price-dividend ratio equal to the one dictated by the present value stock price. 


\section{Estimates}

Tables 2.5a and 2.5b present maximum likelihood estimates of the models, Implied $\mathrm{AR}(1)$ and $\mathrm{AR}(0)$ Model, described above. Each table presents estimates of all four SubModels A-D.

For the unrestricted Sub-Model A of the Implied AR(1) Model, we obtain estimates of $b_{0}=0.67(0.79)$ and $b_{1}=0.29(0.63)$ for the DJIA (S\&P 500) series as reported in Table 2.5a. Estimated variance of the model error is now just over half that of the series reported in Table 2.1. Minimum AIC criterion selects Sub-Model B (Sub-Model A) of Implied AR(1) Model as best for the DJIA (S\&P 500) series among the four Sub-Models, suggesting the importance of the bubble component in rationalizing movements in both series.

For the unrestricted Sub-Model A of the Implied AR(0) Model, we obtain estimates of $b_{0}=0.92(1.21)$ and $b_{1}=0.05(0.11)$ for the DJIA (S\&P 500) series as reported in Table 2.5b. Thus, the estimated slope coefficients on the bubble component are now lower than those for the AR(1) model. The slope coefficients on the fundamental component imply that the estimated fundamental present values (i.e., the product of $b_{0}$ and $\kappa$ ) are very similar to the DJIA (S\&P 500) constant, theoretical price-dividend ratios $\kappa$ of 17.102 (15.02) reported in the bottom panel of Table 2.3, but are also much lower than empirically observed mean price-dividend ratios of 28.30 (29.79) reported in Table 2.1. For comparison, we note that the estimated fundamental present values and slope coefficients on the bubble component obtained here are higher for the former and lower for the latter than the estimates of roughly 14 and at least 0.26 , respectively as reported in Froot and Obstfeld (1991) and Bidarkota and Dupoyet (2007) for the Gaussian AR(0) dividend growth rate. Minimum AIC criterion once again selects Sub-Model B (Sub-Model A) of the Implied AR(0) Model as best for the DJIA (S\&P 500) series among the four SubModels. 
Among all the models estimated in Tables 2.5a and 2.5b, minimum AIC criterion selects Sub-Model B of Implied AR(0) Model (Sub-Model A of Implied AR(1) Model) as best for the DJIA (S\&P 500) series. Thus, the broader stock market index favors the AR(1) model developed here, whereas the blue chip index favors the Froot and Obstfeld (1991) model by this criterion.

Figures 2.5 and 2.6 plot the observed price-dividend ratios and prices, along with the fitted values of the fundamental and bubble components from Sub-Model A of both the Implied AR(1) and AR(0) Models, respectively. The contribution of the fundamental present value component alone in accounting for variation in observed ratios and prices is vividly insufficient in both figures. Taking the bubble component into account provides a much better fit to the $\mathrm{P} / \mathrm{D}$ ratios and stock prices.

Figures 2.7 and 2.8 compare the performance of the fundamental present value component alone and fundamental plus bubble components, respectively, between Sub-Model A of the Implied $A R(1)$ and $A R(0)$ Models. Visually, the empirical performance of the two models is indistinguishable from one another.

In summary, while there is support for the AR(1) model developed here, particularly for the S\&P 500 series, as compared to the AR(0) model of Froot and Obstfeld (1991), both models provide very similar performance when judged in terms of implied fluctuations in the $\mathrm{P} / \mathrm{D}$ ratios and stock prices.

\section{Tests of Hypotheses}

A test of the present value model can be conducted by testing for the null hypothesis that $b_{0}=1$ and $b_{1}=0$ for Sub-Model A of the Implied AR(1) Model described in Equation (2.22) above. The alternative hypothesis of $b_{1}>0$ and/or $b_{0} \neq 1$ is a rejection of the present value model. The null hypothesis of no intrinsic bubbles implies that $b_{1}=0$ in Equation (2.22). $b_{1}>0$ implies rejection of the absence of bubbles. These null and alter- 
native hypotheses are identical in the case of Sub-Model A of the Implied AR(0) Model estimated by Froot and Obstfeld (1991).

Tables 2.6a and 2.6b report likelihood ratio (LR) tests for various hypotheses of interest. In Table 2.6a, under an AR(1) process for dividend growth rates, LR tests for the two hypotheses, $b_{1}=0$ and joint hypothesis $b_{0}=1$ and $b_{1}=0$ are unequivocally rejected. Thus, we can reject the null hypothesis of no bubbles for this model. LR test of $b_{0}=1$ is rejected for the DJIA, but not for the S\&P 500 .

For comparison, in Table 2.6b under an $\mathrm{AR}(0)$ process for dividend growth rates, we find from Sub-Model A of the Implied AR(0) Model that, as in Table 2.6a, LR tests for the single hypothesis, $b_{1}=0$ and joint hypothesis, $b_{0}=1$ and $b_{1}=0$ are rejected. Like the Implied AR(1) Model, the no bubbles hypothesis is rejected for this model as well. However, in contrast to the results in Table 2.6a, the null hypothesis of $b_{0}=1$ is rejected for the $\mathrm{S} \& \mathrm{P}$ 500 , but not for the DJIA.

To provide support of optimal model selection, Table 2.6c shows the LR test results between the two models. The null hypothesis of Implied $\operatorname{AR}(0)$ as the optimal fit is unarguably rejected. In other words, there is evidence that favors the Implied AR(1) over the Implied AR(0) model. This is a critical result which lends support for our model extension from previous work.

\section{Free Models - A Purely Econometric Specification}

We also estimate an alternative version of the above two models, referred to as Free AR(1) Model and Free AR(0) Model, respectively. In these models, the values of the bubble component parameters are no longer set equal to the implied parameter values reported in the respective panels of Table 2.3, but are instead estimated freely along with the rest of the regression parameters of the econometric model. 
Thus, the most general model is the Free AR(1) Model. We also have the Free $\operatorname{AR}(0)$ Model. Among these two models, here too, we estimate an unrestricted version, which is referred to as Sub-Model A, and three restricted versions, referred to as Sub-Models B, C, and D. The restrictions describing these three versions of the models are identical to those described earlier.

Tables $2.5 \mathrm{c}$ and $2.5 \mathrm{~d}$ present maximum likelihood estimates of these two models, Free AR(1) Model and Free AR(0) Model, described above. Each table presents estimates of all four Sub-Models A-D. In Table 2.5c, under the AR(1) process for dividends growth and free estimation of the nonlinear bubble components, the $\lambda$ parameter estimates are 1.713 and 1.741 for the two data series. The estimate for $\lambda$ is similar to the DJIA implied parameter value in Panel A of Table 2.3. However, the S\&P $500 \lambda$ estimate of 1.741 is quite lower than the implied parameter value of 2.148 reported there. The parameter $h$ has been constrained to 0 in order to comply with the non-negativity assumption in our theoretical model.

Figures 2.9 and 2.10 plot observed P/D ratios and prices, along with fitted values of the fundamental and bubble components from Sub-model A of the Free AR(1) and AR(0) Model, respectively. As seen earlier in Figures 2.5 and 2.6 for the Implied AR(1) and AR(0) Models, the fundamental component alone does not track observed index P/D ratios and prices adequately. Adding the bubble component allows the model to track the observed data remarkably better. Figure 2.11 compares the fit of both the fundamental and bubble components to the observed ratios and prices of Sub-model A between Free AR(1) and AR(0) Models. Visually, the empirical performance of both models is indistinguishable from one another. Table 2.6c confirms this indistinction as the LR test does not reject the hypothesis that Free AR(0) Model is the optimal model. Put another way, Free AR(1) Model is no better for predictive performance than that of the Free AR(0) Model. 
For the sake of completeness, we visually and quantitatively compare Implied and Free AR(1) Models and Implied and Free AR(0) Models in Figures 2.12 and 2.13, respectively. Again, visually the models are not considerably different from each other. Table 2.6d confirms this observation for the DJIA results as the LR tests do no reject the null Implied AR(1) and AR(0) Models, respectively; however, for the S\&P 500 results in Table 6e, we can easily reject Implied $A R(1)$ and $A R(0)$ Models. In other words, under both $\mathrm{AR}(1)$ and $\mathrm{AR}(0)$ process-driven specifications, we found no evidence that the theoretical implied values of $\lambda$ and $h$ in Table 2.3 are not accurate estimates of the degree of non-linearity in the price-dividend data for DJIA, but we can statistically significantly reject the S\&P 500 implied parameter values.

\subsubsection{Summary of Results and Model Comparison}

The nonlinear price-dividend ratio regression results reported in Tables 5 and 6, and discussed in subsection 2.4.6 above, indicate that we can conclusively reject the null hypothesis of the absence of intrinsic bubbles across all four models, Implied and Free, $\mathrm{AR}(1)$ and $\mathrm{AR}(0)$, considered here. This inference is robust, regardless of whether or not we restrict $b_{0}$ to 1 . It aligns with the conclusions of Froot and Obstfeld (1991) and Bidarkota and Dupoyet (2007). Further substantiating the regression results, Figures 2.5 and 2.6 and 2.9 and 2.10 clearly demonstrate that the fundamental and bubble components together track the observed stock indices more closely than the fundamental present value component alone.

We conduct additional model analysis to determine a performance leader. When using implied parameter values, the nonlinear model in which dividend growth rates follow an $A R(1)$ process outperforms a model driven by an $A R(0)$ process. This shows the improvement in the performance of the extension considered here when compared 
to the model in Froot and Obstfeld (1991). On the other hand, the two models are indistinguishable when the bubble parameters are freely estimated.

Moreover, for both data series, a nonlinear model in which we use the implied parameter values and in which dividends growth follows either an $\operatorname{AR}(1)$ process or $\operatorname{AR}(0)$ process is statistically superior than that of the same model except in which the bubble components are freely estimated.

We could not find distinct differences between each model visually as Figures 2.7 and 2.8 and 2.11-2.13 show. However, LR tests reported in Tables 2.6a-2.6e allow us to infer quantitatively that Implied AR(1) Model is statistically superior to Implied AR(0) Model. This demonstrates the usefulness of the extension to the work in Froot and Obstfeld (1991) considered here. The Free $\operatorname{AR}(1)$ and $A R(0)$ Models seem to be equivalent, and Implied AR(1) Model (AR(0) Model) is statistically superior to Free $\mathrm{AR}(1)$ Model $(\mathrm{AR}(0)$ Model).

\subsection{Conclusions}

We extend the constant discount factor model with intrinsic bubbles developed in Froot and Obstfeld (1991) to account for serial correlation in the dividend growth rate. We derive an exact analytical expression for both the present value stock price and an intrinsic bubble component when dividend growth rates evolve as a Gaussian first-order autoregressive process. We estimate the model with two sets of annual U.S. stock price and dividends data, namely the DJIA and the S\&P 500 series, over the last century. We compare the results with the benchmark specification under which dividend growth rates follow an AR(0) process, as in Froot and Obstfeld (1991).

Hypotheses tests reject an $\mathrm{AR}(0)$ process for dividend growth rates in favor of an $\mathrm{AR}(1)$ process for both data series. Likelihood ratio tests also favor the AR(1)-based 
model developed here for price-dividends ratios to the AR(0)âASŞbased model considered in Froot and Obstfeld (1991). Information-based model selection criteria favor the $\mathrm{AR}(1)$ model developed here, particularly for the S\&P 500 series, as compared to the AR(0) model of Froot and Obstfeld (1991). Nonetheless, the implied P/D ratios and stock prices from the two models are visually indistinguishable from one another.

Hypotheses tests also reject the absence of a bubble component in both series. This inference is robust to whether or not the parameters governing the intrinsic bubbles process are restricted to values implied by our model or freely estimated. Incorporating the bubble component into our model provides a significant improvement in fit to observed $\mathrm{P} / \mathrm{D}$ ratios and stock prices as compared to the present value stock prices alone. 
Table 2.1: Summary Statistics

\begin{tabular}{|c|c|c|c|c|c|c|c|c|c|c|c|}
\hline Variable & Index & $\begin{array}{l}\text { Sample } \\
\text { Size n }\end{array}$ & Mean & Variance & Median & Min & Max & FOAC & Skewness & Kurtosis & $\begin{array}{l}\text { Test for } \\
\text { Nor- } \\
\text { mality }\end{array}$ \\
\hline \multirow{4}{*}{$\begin{array}{l}\text { Dividend } \\
\text { Growth Rate }\end{array}$} & DJIA & 97 & 0.021 & 0.020 & 0.029 & -0.545 & 0.502 & 0.228 & $-0.587^{* *}$ & $7.256^{* * *}$ & $78.768^{* * *}$ \\
\hline & & & $(0.015)$ & $(0.003)$ & & & & & $(0.018)$ & $(0.000)$ & $(0.000)$ \\
\hline & S\&P 500 & 118 & 0.017 & 0.012 & 0.024 & -0.422 & 0.397 & 0.234 & $-0.795^{* * *}$ & $7.267^{* * *}$ & $101.952^{* * *}$ \\
\hline & & & $(0.010)$ & $(0.002)$ & & & & & $(0.001)$ & $(0.000)$ & $(0.000)$ \\
\hline \multirow{4}{*}{ P/D Ratio } & DJIA & 97 & 28.300 & 120.658 & 25.860 & 13.435 & 62.353 & 0.928 & $1.159^{* * *}$ & $4.047^{*}$ & $26.152^{* * *}$ \\
\hline & & & (1.115) & $(17.400)$ & & & & & $(0.000)$ & $(0.052)$ & $(0.000)$ \\
\hline & S\&P 500 & 118 & 29.790 & 240.193 & 25.320 & 10.459 & 85.296 & 0.919 & $1.519^{* * *}$ & $5.003^{* * *}$ & $65.097^{* * *}$ \\
\hline & & & (1.440) & $(31.700)$ & & & & & $(0.000)$ & $(0.003)$ & $(0.000)$ \\
\hline
\end{tabular}

Numbers in parentheses for mean and variance are their standard errors. FOAC is the first-order autocorrelation coefficient. Numbers in parentheses for skewness and kurtosis are the p-values. The null hypotheses are no skewness and no excess kurtosis, respectively. Test for normality gives the Jarque-Bera test statistic and its $\mathrm{p}$-value in parentheses. ${ }^{*} \mathrm{p}<0.1 ;{ }^{* *} \mathrm{p}<0.05 ;{ }^{* * *} \mathrm{p}<0.01$. 
Table 2.2: Dividend Growth Rate Process Estimates

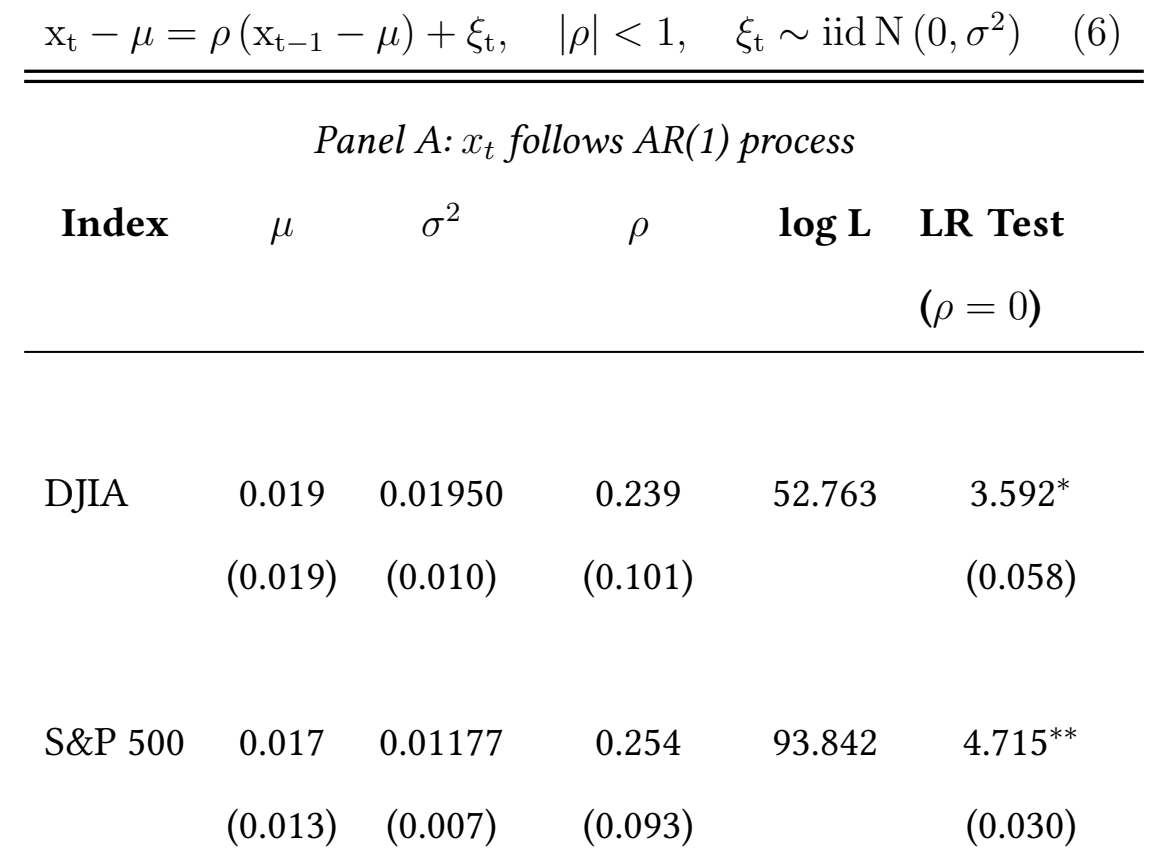

Panel B: $x_{t}$ follows $A R(0)$ process

\begin{tabular}{lcccc} 
Index & $\mu$ & $\sigma^{2}$ & $\rho$ & $\log \mathbf{L}$ \\
\hline \multirow{2}{*}{ DJIA } & 0.021 & 0.02047 & 0 & 50.967 \\
& $(0.015)$ & $(0.010)$ & (restricted) \\
& & & & \\
S\&P 500 & 0.017 & 0.01242 & 0 & 91.484 \\
& $(0.010)$ & $(0.007)$ & (restricted) \\
\hline
\end{tabular}

Maximum likelihood estimates of Eq. (1.6) for the dividend growth rate process are reported in Panel A. Maximum likelihood estimates of a restricted model with $\rho=0$ are reported in Panel B. Numbers in parentheses for the parameter estimates are their standard errors. LR Test in the last column gives the likelihood ratio (LR) test statistic. $\mathrm{P}$-values from $\chi^{2}$ distribution with appropriate $\mathrm{df}$ are in parentheses. ${ }^{*} \mathrm{p}<0.1 ;{ }^{* *} \mathrm{p}<0.05 ;{ }^{* * *} \mathrm{p}<0.01$. 
Table 2.3: Implied Parameter Values

\begin{tabular}{|c|c|c|c|c|}
\hline \multicolumn{5}{|c|}{ Panel A: $x_{t}$ follows $A R(1)$ process } \\
\hline Index & $\mathbf{r}$ & $\lambda$ & $\mathbf{h}$ & $\begin{array}{l}\text { Time- } \\
\text { varying } \\
\kappa \text { (mean) }\end{array}$ \\
\hline DJIA & 0.086 & 1.772 & 0.557 & 17.210 \\
\hline S\&P 500 & 0.086 & 2.148 & 0.730 & 15.501 \\
\hline \multicolumn{5}{|c|}{ Panel B: $x_{t}$ follows $A R(0)$ process } \\
\hline Index & $\mathbf{r}$ & $\lambda$ & $\mathbf{h}$ & constant \\
\hline & & & & $\kappa$ \\
\hline DJIA & 0.086 & 2.062 & & 17.102 \\
\hline S\&P 500 & 0.086 & 2.609 & & 15.020 \\
\hline
\end{tabular}


Table 2.4: Description of various regression specifications for nonlinear $\mathrm{P} / \mathrm{D}$ ratio models in Tables 2.5-2.6

\begin{tabular}{|c|c|}
\hline Model & Description \\
\hline Implied AR(1) Model & $\begin{array}{l}\text { This is the nonlinear price-dividend regression specification in which we set } \lambda \text { and h equal } \\
\text { to the implied parameter values in Table } 2.3 \text { and in which dividends growth, } x_{t} \text { follows an } \\
\qquad A R(1) \text { process. }\end{array}$ \\
\hline Implied $A R(0)$ Model & $\begin{array}{l}\text { This is the nonlinear price-dividend regression specification in which we set } \lambda \text { and h equal } \\
\text { to the implied parameter values in Table } 2.3 \text { and in which dividends growth, } x_{t} \text { follows an } \\
\qquad A R(0) \text { process. }\end{array}$ \\
\hline Free AR(1) Model & $\begin{array}{l}\text { This is the nonlinear price-dividend regression specification in which we estimate } \lambda \text { and } \mathrm{h} \\
\text { freely and in which dividends growth, } x_{t} \text { follows an AR(1) process. }\end{array}$ \\
\hline Free $A R(0)$ Model & $\begin{array}{l}\text { This is the nonlinear price-dividend regression specification in which we estimate } \lambda \text { and } \mathrm{h} \\
\text { freely and in which dividends growth, } x_{t} \text { follows an AR(0) process. }\end{array}$ \\
\hline $\begin{array}{ll} & \text { Sub-Model } \\
\text { A } \\
\text { B } \\
\text { C } \\
\text { D }\end{array}$ & $\begin{array}{c}\text { Description } \\
\text { Unrestricted model } \\
\text { Semi-restricted model }\left(b_{0}=1\right) \\
\text { Semi-restricted model }\left(b_{1}=0\right) \\
\text { Restricted model }\left(b_{1}=0 \text { and } b_{0}=1\right)\end{array}$ \\
\hline
\end{tabular}


Table 2.5a: Maximum likelihood estimation of Implied AR(1) Model

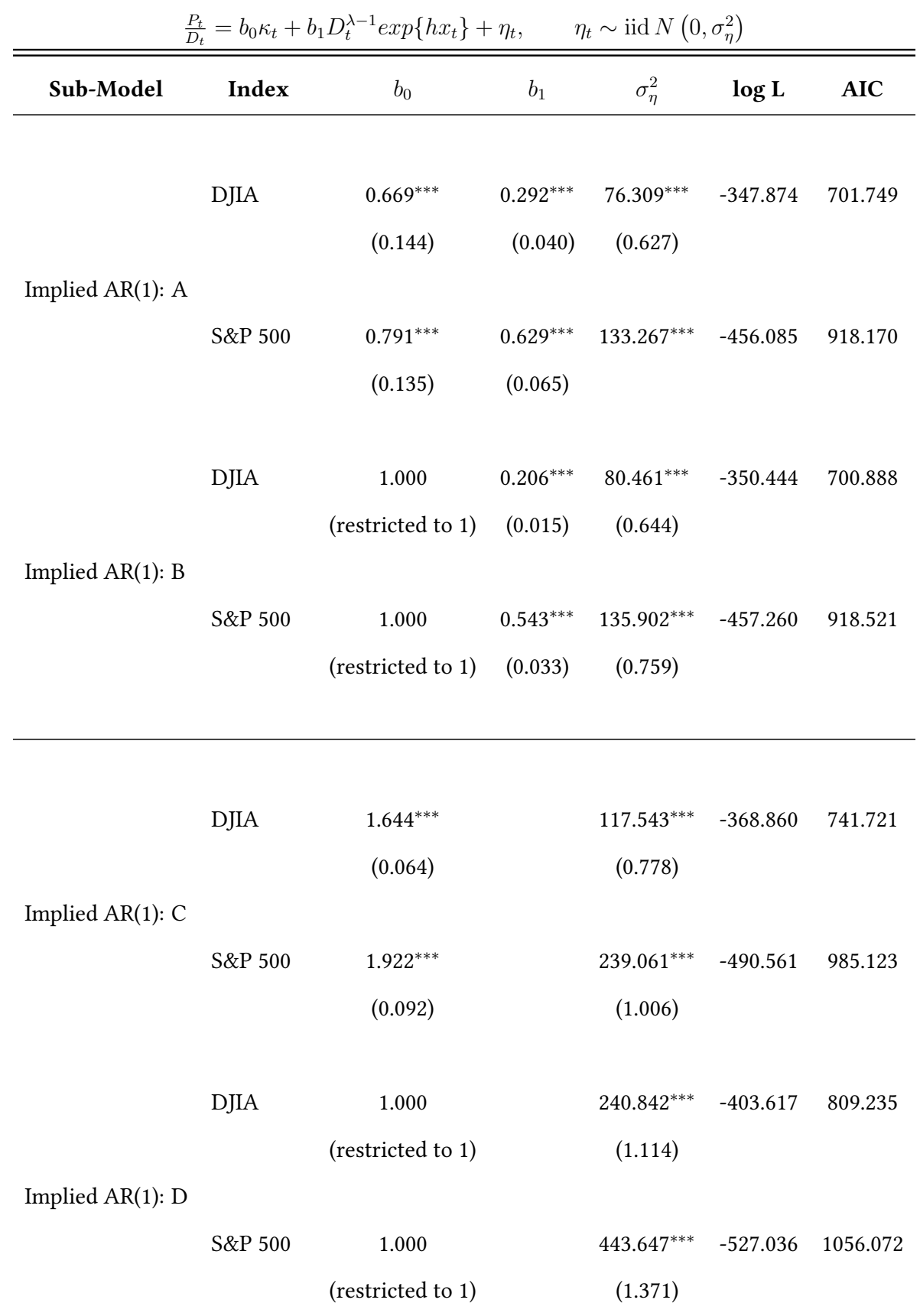

Implied AR(1) Model is the nonlinear price-dividend regression specification in which we set $\lambda$ and $\mathrm{h}$ equal to the implied parameter values in Table 2.3 and in which dividends growth, $x_{t}$ follows an AR(1) process. Sub-models A-D: Sub-model A estimates $b_{0}$ and $b_{1}$ freely; Sub-model B restricts $b_{0}=1$; Sub-model C restricts $b_{1}=0$; and Sub-model D restricts both $b_{0}=1$ and $b_{1}=0$. Numbers in parentheses for the parameter estimates are their standard errors. ${ }^{*} \mathrm{p}<0.1 ;{ }^{* *} \mathrm{p}<0.05 ;{ }^{* * *} \mathrm{p}<0.01$. 
Table 2.5b: Maximum likelihood estimation of Implied AR(0) Model

\begin{tabular}{|c|c|c|c|c|c|c|}
\hline & $\frac{P_{t}}{D_{t}}=b$ & $+b_{1} D_{t}^{\lambda-1}+v_{t}$ & $v_{t} \sim \mathrm{i}$ & $\mathrm{d} N\left(0, \sigma_{v}^{2}\right)$ & & \\
\hline Sub-Model & Index & $b_{0}$ & $b_{1}$ & $\sigma_{v}^{2}$ & $\log L$ & AIC \\
\hline \multirow{5}{*}{ Implied AR(0): } & DJIA & $0.923^{* * *}$ & $0.047^{* * *}$ & $75.024^{* * *}$ & -347.049 & 700.098 \\
\hline & & $(0.111)$ & $(0.006)$ & $(0.622)$ & & \\
\hline & & & & & & \\
\hline & S\&P 500 & $1.208^{* * *}$ & $0.105^{* * *}$ & $139.435^{* * *}$ & -458.750 & 923.501 \\
\hline & & $(0.112)$ & $(0.011)$ & & & \\
\hline \multirow{5}{*}{ Implied AR(0): B } & DJIA & 1.000 & $0.043^{* * *}$ & $75.405^{* * *}$ & -347.297 & 698.593 \\
\hline & & (restricted to 1 ) & $(0.003)$ & $(0.623)$ & & \\
\hline & & & & & & \\
\hline & S\&P 500 & 1.000 & $0.121^{* * *}$ & $143.575^{* * *}$ & -460.482 & 924.963 \\
\hline & & (restricted to 1 ) & $(0.008)$ & $(0.780)$ & & \\
\hline \multirow{5}{*}{ Implied $A R(0): C$} & DJIA & $1.655^{* * *}$ & & $118.990^{* * *}$ & -369.421 & 742.841 \\
\hline & & $(0.065)$ & & $(0.783)$ & & \\
\hline & & & & & & \\
\hline & S\&P 500 & $1.984^{* * *}$ & & $240.170^{* * *}$ & -490.834 & 985.667 \\
\hline & & $(0.095)$ & & $(1.009)$ & & \\
\hline \multirow{5}{*}{ Implied AR(0): $\mathrm{D}$} & DJIA & 1.000 & & $244.412^{* * *}$ & -404.331 & 810.663 \\
\hline & & (restricted to 1 ) & & $(1.122)$ & & \\
\hline & & & & & & \\
\hline & S\&P 500 & 1.000 & & $458.418^{* * *}$ & -528.976 & 1059.951 \\
\hline & & (restricted to 1 ) & & (1.394) & & \\
\hline
\end{tabular}

Implied $\mathrm{AR}(0)$ Model is the nonlinear price-dividend regression specification in which we set $\lambda$ and $\mathrm{h}$ equal to the implied parameter values in Table 2.3 and in which dividends growth, $x_{t}$ follows an AR(0) process. Sub-model A estimates $b_{0}$ and $b_{1}$ freely; Sub-model B model restricts $b_{0}=1$; Sub-model C restricts $b_{1}=0$; and Sub-model D restricts both $b_{0}=1$ and $b_{1}=0$. Numbers in parentheses for the parameter estimates are their standard errors. ${ }^{*} \mathrm{p}<0.1 ;{ }^{* *} \mathrm{p}<0.05 ;{ }^{* * *} \mathrm{p}<0.01$. 
Table 2.5c: Maximum likelihood estimation of Free AR(1) Model

\begin{tabular}{|c|c|c|c|c|c|c|c|c|}
\hline \multicolumn{5}{|c|}{$\frac{P_{t}}{D_{t}}=b_{0} \kappa_{t}+b_{1} D_{t}^{\lambda-1} \exp \left\{h x_{t}\right\}+\eta_{t}}$, & \multicolumn{4}{|c|}{$\eta_{t} \sim \operatorname{iid} N\left(0, \sigma_{\eta}^{2}\right)$} \\
\hline Sub-Model & Index & $b_{0}$ & $b_{1}$ & $\lambda$ & $\mathbf{h}$ & $\sigma_{\eta}^{2}$ & $\log L$ & AIC \\
\hline & DJIA & 0.537 & 0.462 & $1.713^{* * *}$ & 0.000 & $74.372^{* * *}$ & -346.626 & 703.253 \\
\hline & & $(0.563)$ & $(1.056)$ & $(0.339)$ & $(0.399)$ & $(0.620)$ & & \\
\hline \multicolumn{9}{|l|}{ Free $A R(1): A$} \\
\hline & S\&P 500 & 0.000 & 3.665 & $1.741^{* * *}$ & 0.000 & $120.674^{* * *}$ & -450.227 & 910.455 \\
\hline & & $(0.561)$ & (2.994) & $(0.186)$ & $(0.384)$ & $(0.731)$ & & \\
\hline & DJIA & 1.000 & 0.036 & $2.095^{* * *}$ & 0.000 & $75.522^{* * *}$ & -347.370 & 702.741 \\
\hline & & (restricted to 1 ) & $(0.029)$ & $(0.146)$ & $(0.669)$ & $(0.626)$ & & \\
\hline
\end{tabular}

Free $\operatorname{AR}(1): B$

$\begin{array}{cccccccc}\text { S\&P } 500 & 1.000 & 0.443^{* * *} & 2.222^{* * *} & 0.000 & 131.675^{* * *} & -455.374 & 918.748 \\ & \text { (restricted to 1) } & (0.172) & (0.120) & (0.866) & (0.766) & \end{array}$

\begin{tabular}{|c|c|c|c|c|}
\hline DJIA & $1.644^{* * *}$ & $117.543^{* * *}$ & -368.860 & 741.721 \\
\hline & $(0.064)$ & (0.778) & & \\
\hline
\end{tabular}

Free $\operatorname{AR}(1): C$

$\begin{array}{lcccc}\text { S\&P } 500 & 1.922^{* * *} & 239.061^{* * *} & -490.561 & 985.123 \\ & (0.092) & (1.006) & & \\ & & & & \\ \text { DJIA } & 1.000 & 240.842^{* * *} & -403.617 & 809.235 \\ & \text { (restricted to } 1) & (1.114) & & \end{array}$

Free $\operatorname{AR}(1): D$

\begin{tabular}{|c|c|c|c|}
\hline S\&P 500 & 1.000 & $443.647^{* * *}$ & -527.036 \\
\hline & estricted to 1 ) & (1.371) & \\
\hline
\end{tabular}

Free AR(1) Model is the nonlinear price-dividend regression specification in which we estimate $\lambda$ and $h$ freely and in which dividends growth, $x_{t}$ follows an AR(1) process. Sub-model A estimates $b_{0}$ and $b_{1}$ freely; Sub-model B restricts $b_{0}=1$; Sub-model C restricts $b_{1}=0$; and Sub-model D restricts both $b_{0}=1$ and $b_{1}=0$. Numbers in parentheses for the parameter estimates are their standard errors. ${ }^{*} \mathrm{p}<0.1 ;{ }^{* *} \mathrm{p}<0.05$; ${ }^{* * *} \mathrm{p}<0.01$. 
Table 2.5d: Maximum likelihood estimation of Free AR(0) Model

\begin{tabular}{|c|c|c|c|c|c|c|c|}
\hline Sub-Model & Index & $b_{0}$ & $b_{1}$ & $\lambda$ & $\sigma_{v}^{2}$ & $\log L$ & AIC \\
\hline & \multirow[t]{2}{*}{ DJIA } & 0.576 & 0.391 & $1.739^{* * *}$ & $74.321^{* * *}$ & -346.595 & 701.189 \\
\hline & & $(0.510)$ & $(0.849)$ & $(0.324)$ & $(0.619)$ & & \\
\hline
\end{tabular}

Free $\operatorname{AR}(0): A$

$\begin{array}{lcccccc}\text { S\&P 500 } & 0.000 & 3.665 & 1.741^{* * *} & 120.674^{* * *} & -450.227 & 908.455 \\ & (0.587) & (3.062) & 0.190 & (0.721) & & \\ & & & & & & \\ \text { DJIA } & 1.000 & 0.036 & 2.098^{* * *} & 75.355^{* * *} & -347.264 & 700.527 \\ & \text { (restricted to 1) } & (0.028) & (0.142) & (0.623) & & \end{array}$

Free $\operatorname{AR}(0): B$

\begin{tabular}{|c|c|c|c|c|c|c|}
\hline S\&P 500 & 1.000 & $0.484^{* * *}$ & $2.202^{* * *}$ & $130.253^{* * *}$ & -454.753 & 915.506 \\
\hline & (restricted to 1 ) & $(0.180)$ & $(0.112)$ & $(0.743)$ & & \\
\hline
\end{tabular}

\begin{tabular}{|c|c|c|c|c|}
\hline DJIA & $1.655^{* * *}$ & $118.902^{* * *}$ & -369.421 & 742.841 \\
\hline & $(0.065)$ & $(0.783)$ & & \\
\hline
\end{tabular}

Free $\operatorname{AR}(0): C$

$\begin{array}{lcccc}\text { S\&P 500 } & 1.984^{* * *} & 240.167^{* * *} & -490.834 & 985.667 \\ & (0.095) & (1.009) & & \\ & & & & \\ \text { DJIA } & 1.000 & 244.412^{* * *} & -404.331 & 810.663 \\ & \text { (restricted to } 1) & (1.122) & & \end{array}$

Free $\operatorname{AR}(0): D$

\begin{tabular}{|c|c|c|c|}
\hline S\&P 500 & 1.000 & $458.418^{* * *}$ & -528.976 \\
\hline & (restricted to 1) & $(1.394)$ & \\
\hline
\end{tabular}

Free $\operatorname{AR}(0)$ Model is the nonlinear price-dividend regression specification in which we estimate $\lambda$ and $\mathrm{h}$ freely and in which dividends growth, $x_{t}$ follows an $\mathrm{AR}(0)$ process. Sub-model A estimates $b_{0}$ and $b_{1}$ freely; Sub-model B restricts $b_{0}=1$; Sub-model C restricts $b_{1}=0$; and Sub-model D restricts both $b_{0}=1$ and $b_{1}=0$. Numbers in parentheses for the parameter estimates are their standard errors. ${ }^{*} \mathrm{p}<0.1 ;{ }^{* *} \mathrm{p}<0.05 ;{ }^{* * *} \mathrm{p}<0.01$. 
Table 2.6a: Sub-Model Comparisons: AR(1) Model

\begin{tabular}{|c|c|c|c|c|}
\hline Index & Sub-Model & $\begin{array}{l}\text { LR Test (Null } \\
\left.\mathbf{H}_{0}: b_{0}=\mathbf{1}\right)\end{array}$ & $\begin{array}{l}\text { LR Test (Null } \\
\left.\mathbf{H}_{0}: b_{1}=\mathbf{0}\right)\end{array}$ & $\begin{array}{l}\text { LR Test (Null } \\
\mathbf{H}_{0}: b_{0}=\mathbf{1} \text { and } \\
\left.b_{1}=\mathbf{0}\right)\end{array}$ \\
\hline \multirow{10}{*}{ DJIA } & Implied AR(1): Sub-model A vs Sub-model B & $\begin{array}{l}5.140^{* *} \\
(0.023)\end{array}$ & & \\
\hline & Implied AR(1): Sub-model C vs Sub-model D & $\begin{array}{r}69.514^{* * *} \\
(0.000)\end{array}$ & & \\
\hline & Implied AR(1): Sub-model A vs Sub-model C & & $\begin{array}{r}41.972^{* * *} \\
(0.000)\end{array}$ & \\
\hline & Implied AR(1): Sub-model B vs Sub-model D & & $\begin{array}{r}106.350^{* * *} \\
(0.000)\end{array}$ & \\
\hline & Implied AR(1): Sub-model A vs Sub-model D & & & $\begin{array}{c}111.490^{* * *} \\
(0.000)\end{array}$ \\
\hline & Free AR(1): Sub-model A vs Sub-model B & $\begin{array}{r}1.488 \\
(0.223)\end{array}$ & & \\
\hline & Free AR(1): Sub-model C vs Sub-model D & $\begin{array}{r}69.514^{* * *} \\
(0.000)\end{array}$ & & \\
\hline & Free AR(1): Sub-model A vs Sub-model C & & $\begin{array}{r}44.468^{* * *} \\
(0.000)\end{array}$ & \\
\hline & Free AR(1): Sub-model B vs Sub-model D & & $\begin{array}{r}112.490^{* * *} \\
0.000\end{array}$ & \\
\hline & Free AR(1): Sub-model A vs Sub-model D & & & $\begin{array}{c}113.980^{* * *} \\
(0.000)\end{array}$ \\
\hline \multirow{10}{*}{$S \& P 500$} & Implied AR(1): Sub-model A vs Sub-model B & $\begin{array}{r}2.351 \\
(0.125)\end{array}$ & & \\
\hline & Implied AR(1): Sub-model C vs Sub-model D & $\begin{array}{r}72.949^{* * *} \\
(0.000)\end{array}$ & & \\
\hline & Implied AR(1): Sub-model A vs Sub-model C & & $\begin{array}{r}68.953^{* * *} \\
(0.000)\end{array}$ & \\
\hline & Implied AR(1): Sub-model B vs Sub-model D & & $\begin{array}{r}139.550^{* * *} \\
(0.000)\end{array}$ & \\
\hline & Implied AR(1): Sub-model A vs Sub-model D & & & $\begin{array}{c}141.900^{* * *} \\
(0.000)\end{array}$ \\
\hline & Free AR(1): Sub-model A vs Sub-model B & $\begin{array}{r}10.293^{* * *} \\
(0.001)\end{array}$ & & \\
\hline & Free AR(1): Sub-model C vs Sub-model D & $\begin{array}{r}72.949^{* * *} \\
(0.000)\end{array}$ & & \\
\hline & Free AR(1): Sub-model A vs Sub-model C & & $\begin{array}{r}80.668^{* * *} \\
(0.000)\end{array}$ & \\
\hline & Free AR(1): Sub-model B vs Sub-model D & & $\begin{array}{r}143.320^{* * *} \\
(0.000)\end{array}$ & \\
\hline & Free AR(1): Sub-model A vs Sub-model D & & & $\begin{array}{c}153.620^{* * *} \\
(0.000)\end{array}$ \\
\hline
\end{tabular}


Table 2.6b: Sub-Model Comparisons: AR(0) Model

\begin{tabular}{|c|c|c|c|c|}
\hline Index & Sub-Model & $\begin{array}{l}\text { LR Test (Null } \\
\left.\mathbf{H}_{0}: b_{0}=\mathbf{1}\right)\end{array}$ & $\begin{array}{l}\text { LR Test (Null } \\
\left.\mathbf{H}_{0}: b_{1}=\mathbf{0}\right)\end{array}$ & $\begin{array}{l}\text { LR Test (Null } \\
\mathbf{H}_{0}: b_{0}=\mathbf{1} \text { and } \\
\left.b_{1}=\mathbf{0}\right)\end{array}$ \\
\hline \multirow{10}{*}{ DJIA } & Implied AR(0): Sub-model A vs Sub-model B & $\begin{array}{r}0.495 \\
(0.482)\end{array}$ & & \\
\hline & Implied AR(0): Sub-model C vs Sub-model D & $\begin{array}{c}69.821^{* * *} \\
(0.000)\end{array}$ & & \\
\hline & Implied AR(0): Sub-model A vs Sub-model C & & $\begin{array}{c}44.744^{* * *} \\
(0.000)\end{array}$ & \\
\hline & Implied AR(0): Sub-model B vs Sub-model D & & $\begin{array}{c}114.070^{* * *} \\
(0.000)\end{array}$ & \\
\hline & Implied AR(0): Sub-model A vs Sub-model D & & & $\begin{array}{c}114.560^{* * *} \\
(0.000)\end{array}$ \\
\hline & Free AR(0): Sub-model A vs Sub-model B & $\begin{array}{l}1.338 \\
(0.247)\end{array}$ & & \\
\hline & Free $A R(0)$ : Sub-model C vs Sub-model D & $\begin{array}{c}69.821^{* * *} \\
(0.000)\end{array}$ & & \\
\hline & Free AR(0): Sub-model A vs Sub-model C & & $\begin{array}{c}45.652^{* * *} \\
(0.000)\end{array}$ & \\
\hline & Free AR(0): Sub-model B vs Sub-model D & & $\begin{array}{c}114.140^{* * *} \\
(0.000)\end{array}$ & \\
\hline & Free $\mathrm{AR}(0)$ : Sub-model A vs Sub-model D & & & $\begin{array}{c}115.470^{* * *} \\
(0.000)\end{array}$ \\
\hline \multirow{10}{*}{ S\&P 500} & Implied AR(0): Sub-model A vs Sub-model B & $\begin{array}{l}3.463^{*} \\
(0.063)\end{array}$ & & \\
\hline & Implied AR(0): Sub-model C vs Sub-model D & $\begin{array}{c}76.284^{* * *} \\
(0.000)\end{array}$ & & \\
\hline & Implied AR(0): Sub-model A vs Sub-model C & & $\begin{array}{c}64.166^{* * *} \\
(0.000)\end{array}$ & \\
\hline & Implied AR(0): Sub-model B vs Sub-model D & & $\begin{array}{c}136.990^{* * *} \\
(0.000)\end{array}$ & \\
\hline & Implied AR(0): Sub-model A vs Sub-model D & & & $\begin{array}{c}140.450^{* * *} \\
(0.000)\end{array}$ \\
\hline & Free AR(0): Sub-model A vs Sub-model B & $\begin{array}{l}9.051^{* * *} \\
(0.003)\end{array}$ & & \\
\hline & Free AR(0): Sub-model C vs Sub-model D & $\begin{array}{c}76.284^{* * *} \\
(0.000)\end{array}$ & & \\
\hline & Free AR(0): Sub-model A vs Sub-model C & & $\begin{array}{c}81.212^{* * *} \\
(0.000)\end{array}$ & \\
\hline & Free AR(0): Sub-model B vs Sub-model D & & $\begin{array}{c}148.440^{* * *} \\
(0.000)\end{array}$ & \\
\hline & Free AR(0): Sub-model A vs Sub-model D & & & $\begin{array}{c}157.500^{* * *} \\
(0.000)\end{array}$ \\
\hline
\end{tabular}


Table 2.6c: Full Model Comparisons: AR(1) and AR(0) Models

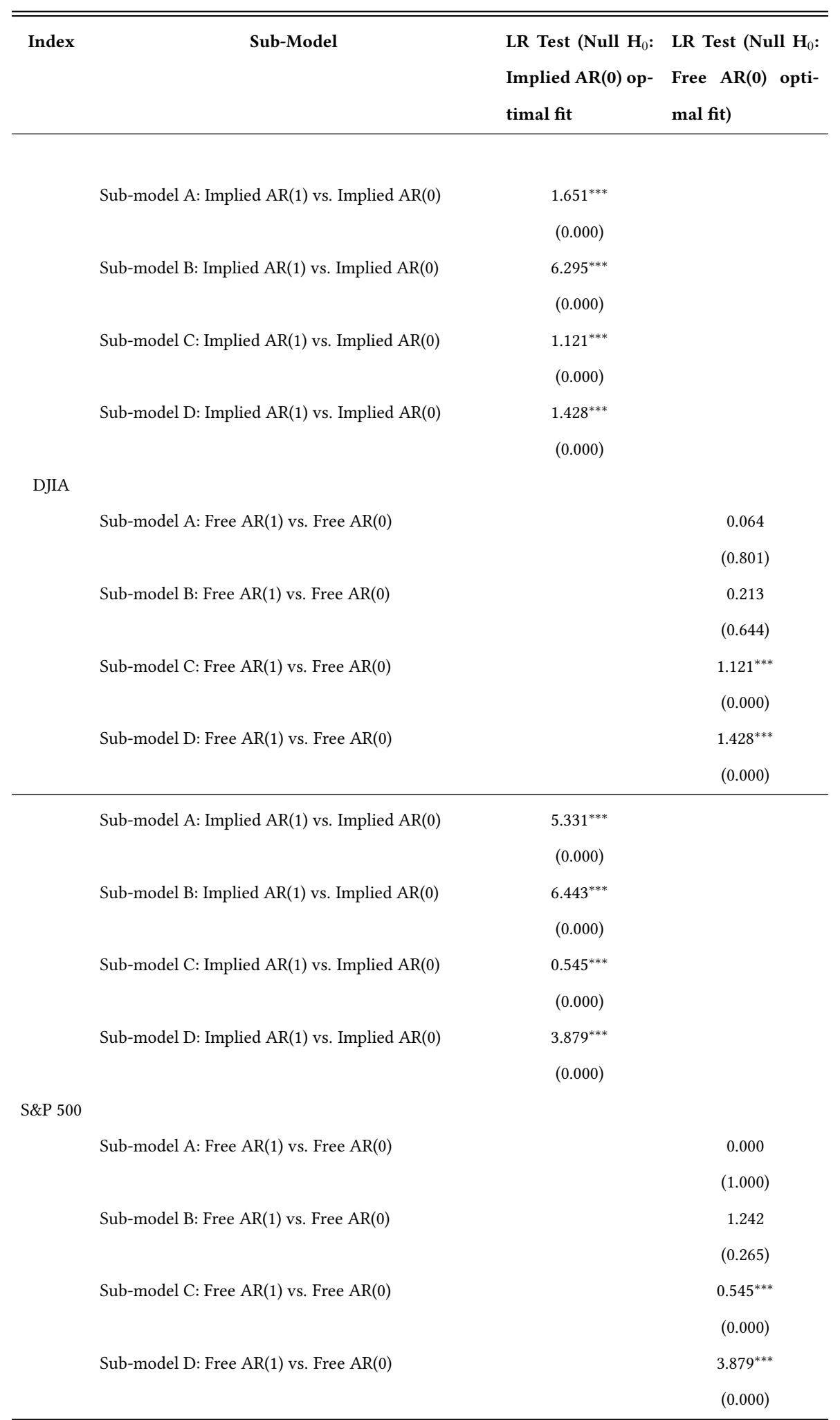

See Table 2.4 for full model and sub-model descriptions. LR Tests give the likelihood ratio (LR) test statistic. $\mathrm{P}$-values from $\chi^{2}$ distribution with appropriate df are in parentheses. ${ }^{*} \mathrm{p}<0.1 ;{ }^{* *} \mathrm{p}<0.05 ;{ }^{* * *} \mathrm{p}<0.01$. 
Table 2.6d: Full Model Comparisons: Implied vs. Free AR(p) Model (DJIA)

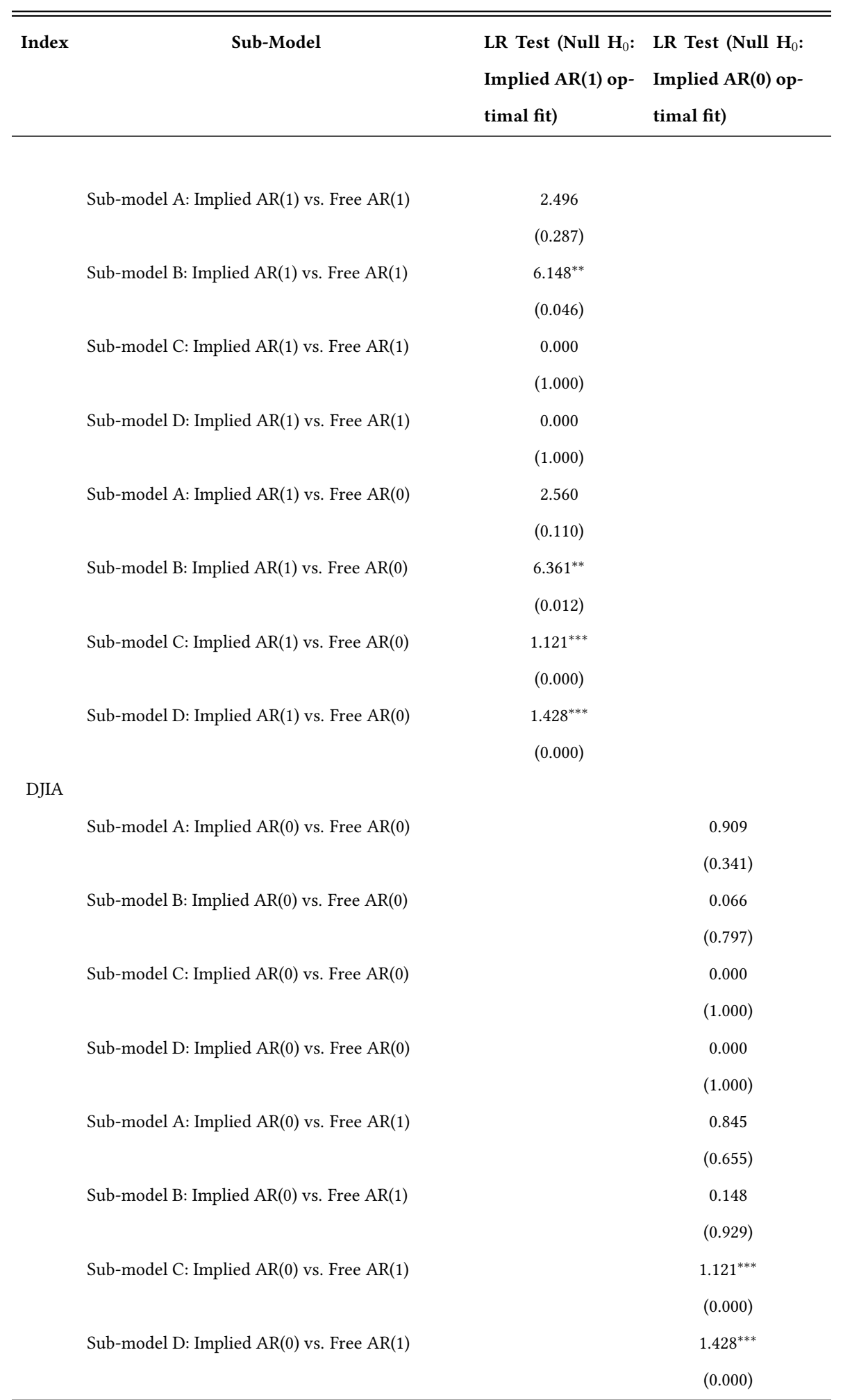

See Table 2.4 for full model and sub-model descriptions. LR Tests give the likelihood ratio (LR) test statistic.

P-values from $\chi^{2}$ distribution with appropriate df are in parentheses. ${ }^{*} \mathrm{p}<0.1 ;{ }^{* *} \mathrm{p}<0.05$; ${ }^{* * *} \mathrm{p}<0.01$. 
Table 2.6e: Model Comparisons: Implied vs. Free AR(p) Model (S\&P 500)

\begin{tabular}{|c|c|c|}
\hline Index & Sub-Model & $\begin{array}{l}\text { LR Test (Null } \\
\text { Implied AR(1 }\end{array}$ \\
\hline & Sub-model A: Implied AR(1) vs. Free AR(1) & $\begin{array}{c}11.715^{* * *} \\
(0.003)\end{array}$ \\
\hline & Sub-model B: Implied AR(1) vs. Free AR(1) & $\begin{array}{c}3.772 \\
(0.152)\end{array}$ \\
\hline & Sub-model C: Implied AR(1) vs. Free AR(1) & $\begin{array}{c}0.000 \\
(1.000)\end{array}$ \\
\hline & Sub-model D: Implied AR(1) vs. Free AR(1) & $\begin{array}{c}0.000 \\
(1.000)\end{array}$ \\
\hline & Sub-model A: Implied AR(1) vs. Free AR(0) & $\begin{array}{c}11.715^{* * *} \\
(0.001)\end{array}$ \\
\hline & Sub-model B: Implied AR(1) vs. Free AR(0) & $\begin{array}{l}5.015^{* *} \\
(0.025)\end{array}$ \\
\hline & Sub-model C: Implied AR(1) vs. Free AR(0) & $\begin{array}{c}0.545^{* * *} \\
(0.000)\end{array}$ \\
\hline & Sub-model D: Implied AR(1) vs. Free AR(0) & $\begin{array}{c}3.879^{* * *} \\
(0.000)\end{array}$ \\
\hline
\end{tabular}

S\&P 500

Sub-model A: Implied AR(0) vs. Free AR(0)

$17.046^{* * *}$

$(0.000)$

Sub-model B: Implied AR(0) vs. Free AR(0)

$11.457^{* * *}$

$(0.001)$

Sub-model C: Implied AR(0) vs. Free AR(0)

0.000

(1.000)

Sub-model D: Implied AR(0) vs. Free AR(0)

0.000

(1.000)

Sub-model A: Implied AR(0) vs. Free AR(1)

$17.046^{* * *}$

$(0.000)$

Sub-model B: Implied AR(0) vs. Free AR(1)

$10.215^{* * *}$

(0.006)

Sub-model C: Implied AR(0) vs. Free AR(1)

$0.545^{* * *}$

$(0.000)$

$3.879^{* * *}$

$(0.000)$

See Table 2.4 for full model and sub-model descriptions. LR Tests give the likelihood ratio (LR) test statistic. P-values from $\chi^{2}$ distribution with appropriate df are in parentheses. ${ }^{*} \mathrm{p}<0.1 ;{ }^{* *} \mathrm{p}<0.05 ;{ }^{* * *} \mathrm{p}<0.01$. 
Figure 2.1: Plots of Dow Jones Industrial Average Data

Real Stock Prices (D.NA)

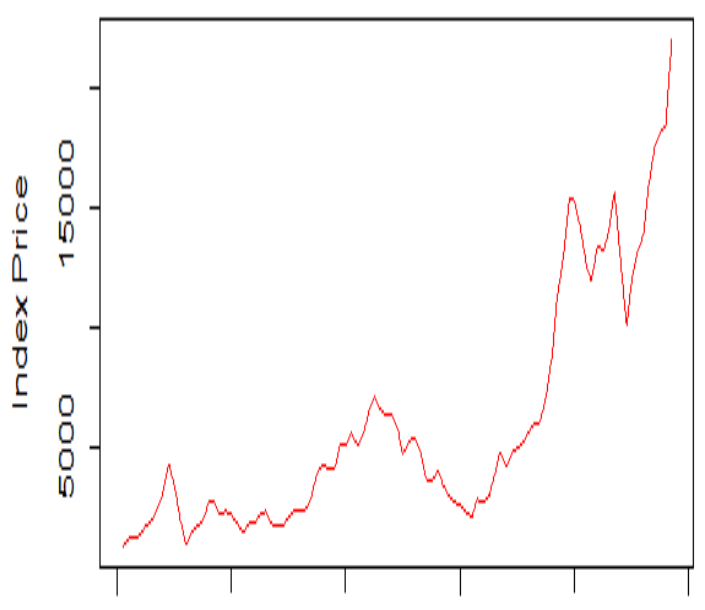

$\begin{array}{llllll}1920 & 1940 \quad 1960 & 1990 & 2000 & 2020\end{array}$

year

(a)

Growth Rates of Real Dividends (DJNA)

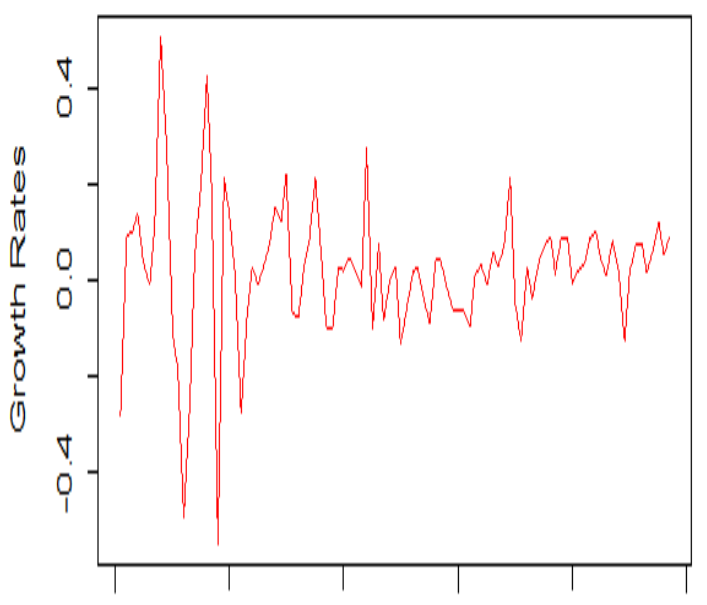

$1920 \quad 1940 \quad 1960 \quad 1990 \quad 2000 \quad 2020$

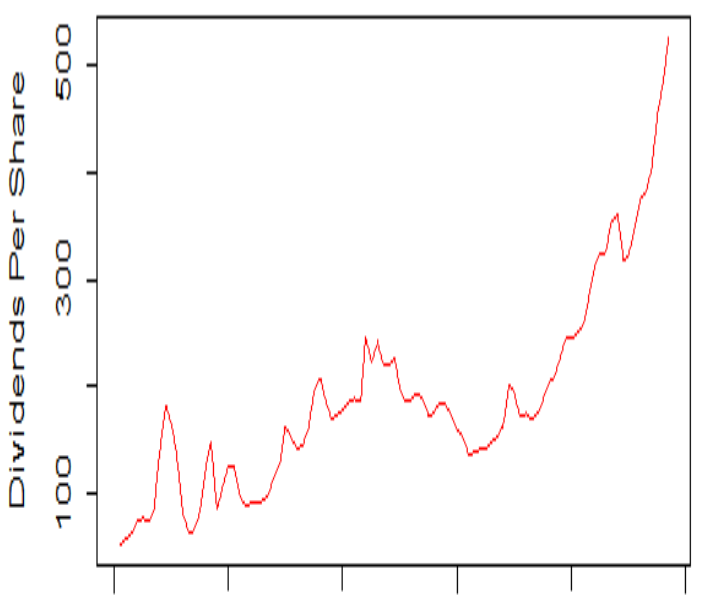

$\begin{array}{llllll}1920 & 1940 & 1960 & 1990 & 2000 & 2020\end{array}$

year

(b)

PID Ratios (DJA)

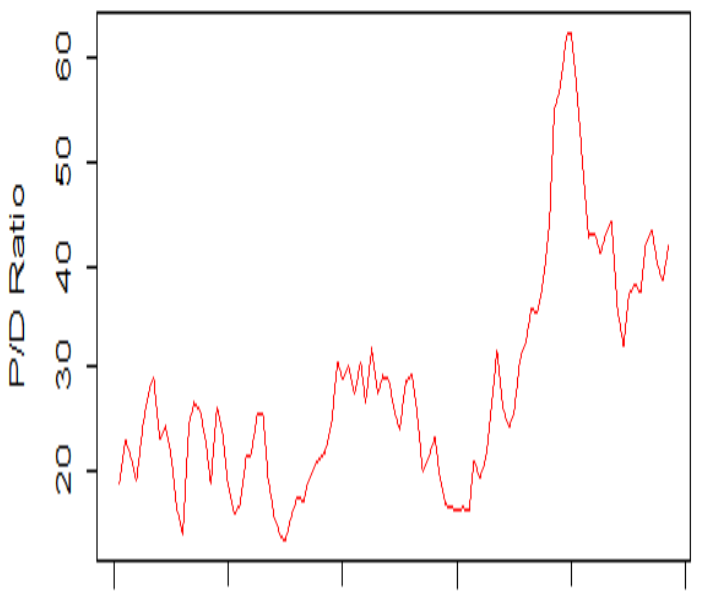

$\begin{array}{llllll}1920 & 1940 \quad 19900 & 1980 & 2000 & 2020\end{array}$

year

year

(c) 
Figure 2.2: Plots of S\&P 500 Data

Real Stock Prices (SSP 500)

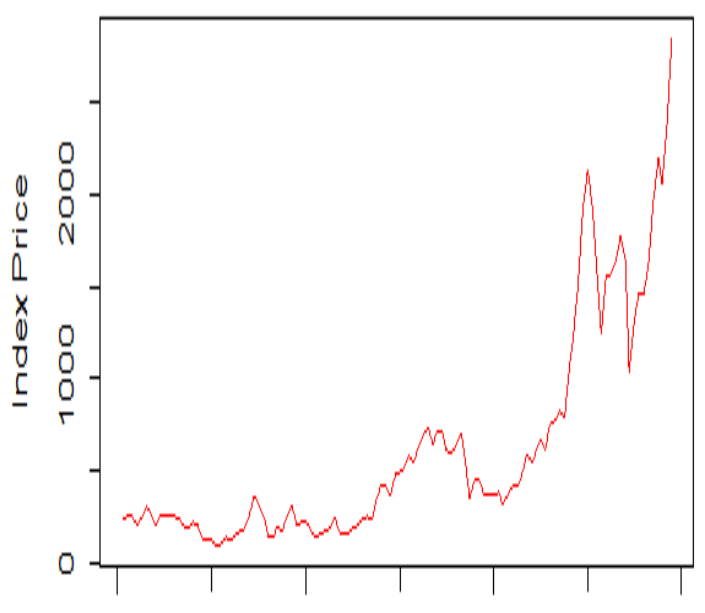

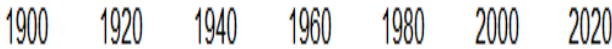

year

(a)

Growth Rates of Real Dividends (SAP 500)

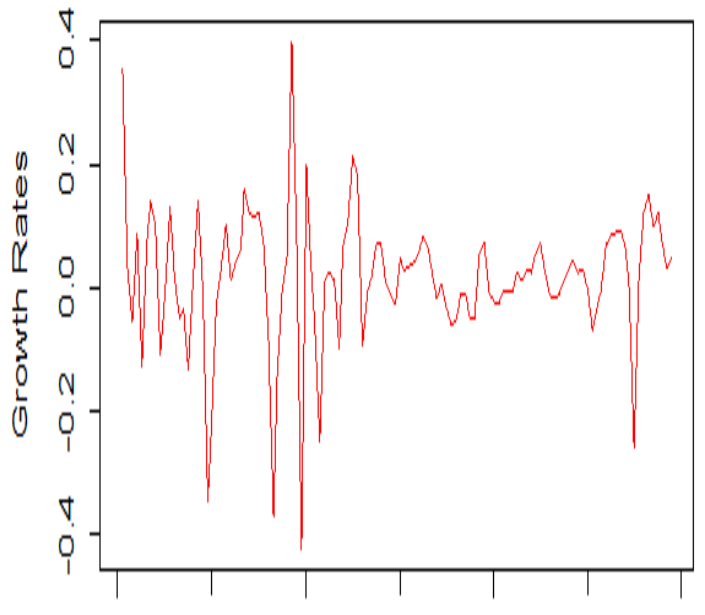

$\begin{array}{llllll}1900 & 1920 & 1940 \quad 1960 & 1980 & 2000 & 2020\end{array}$

yeal

(c)
Real Dividend (SSP 500)

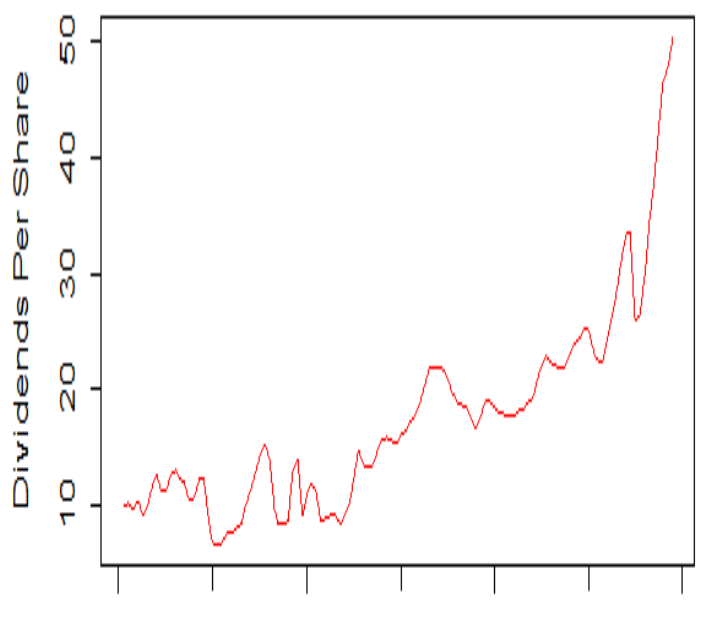

$\begin{array}{lllllll}1900 & 1920 & 1940 & 1960 & 1980 & 2000 & 2020\end{array}$

year

(b)

PIDRatios(SSP 500)

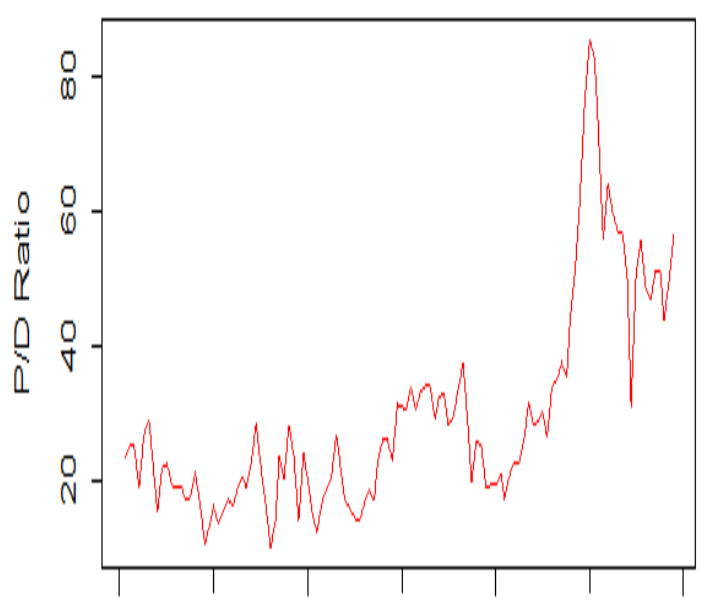

$\begin{array}{lllllll}1900 & 1920 & 1940 & 1960 & 1980 & 2000 & 2020\end{array}$

(d) 
Figure 2.3: Probability distributions of real dividend growth rates

\section{Dividend Growth Rate Per Annum (DJIA)}

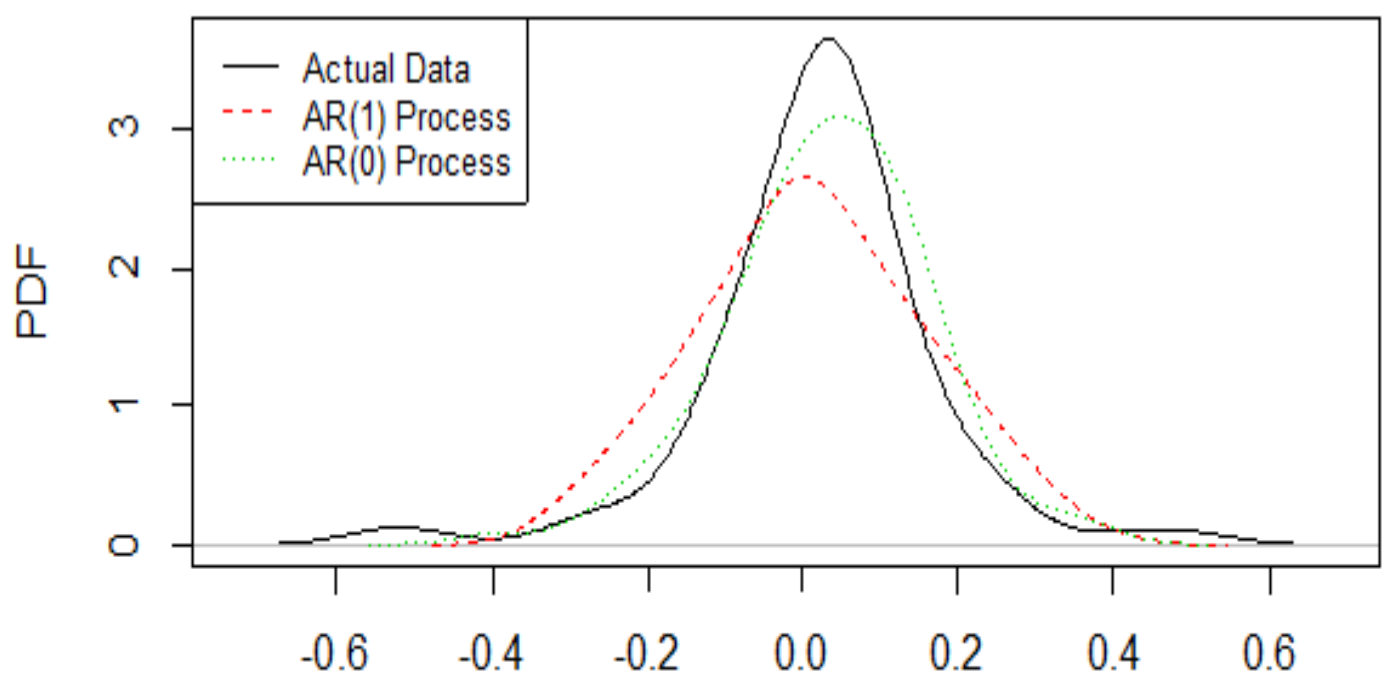

(a)

Dividend Growth Rate Per Annum (S\&P 500)

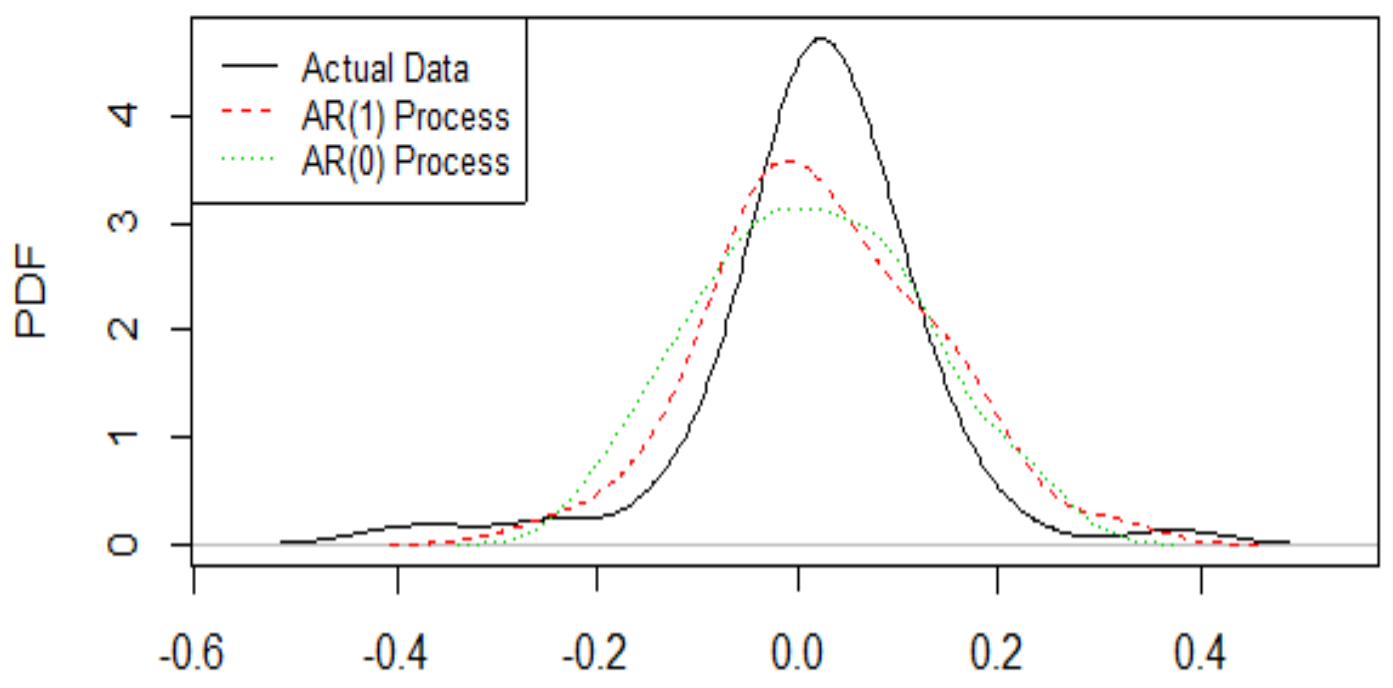

(b) 
Figure 2.4: Plots of Time-varying $\kappa$ and Constant $\kappa$

\section{Kappa (DJIA)}

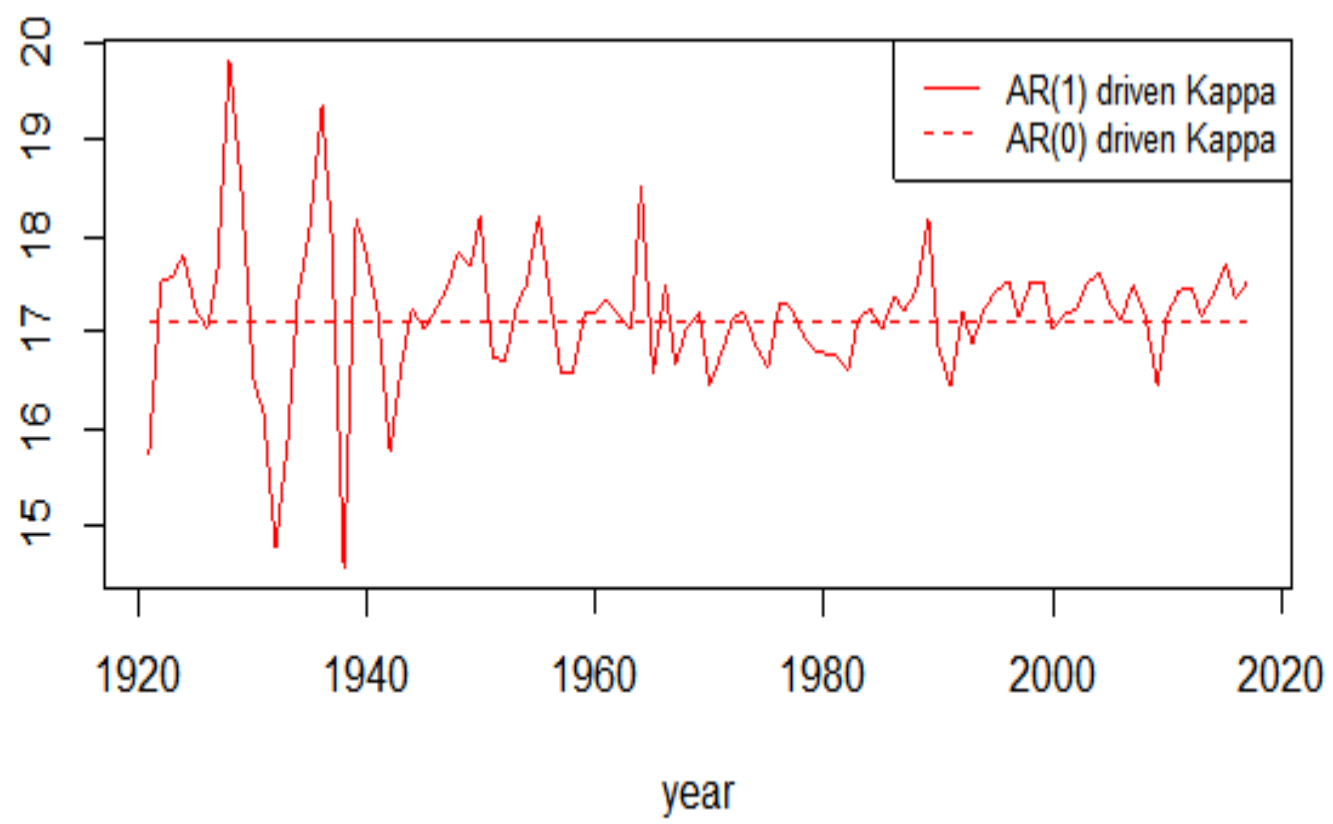

(a)

\section{Kappa (S\&P 500)}

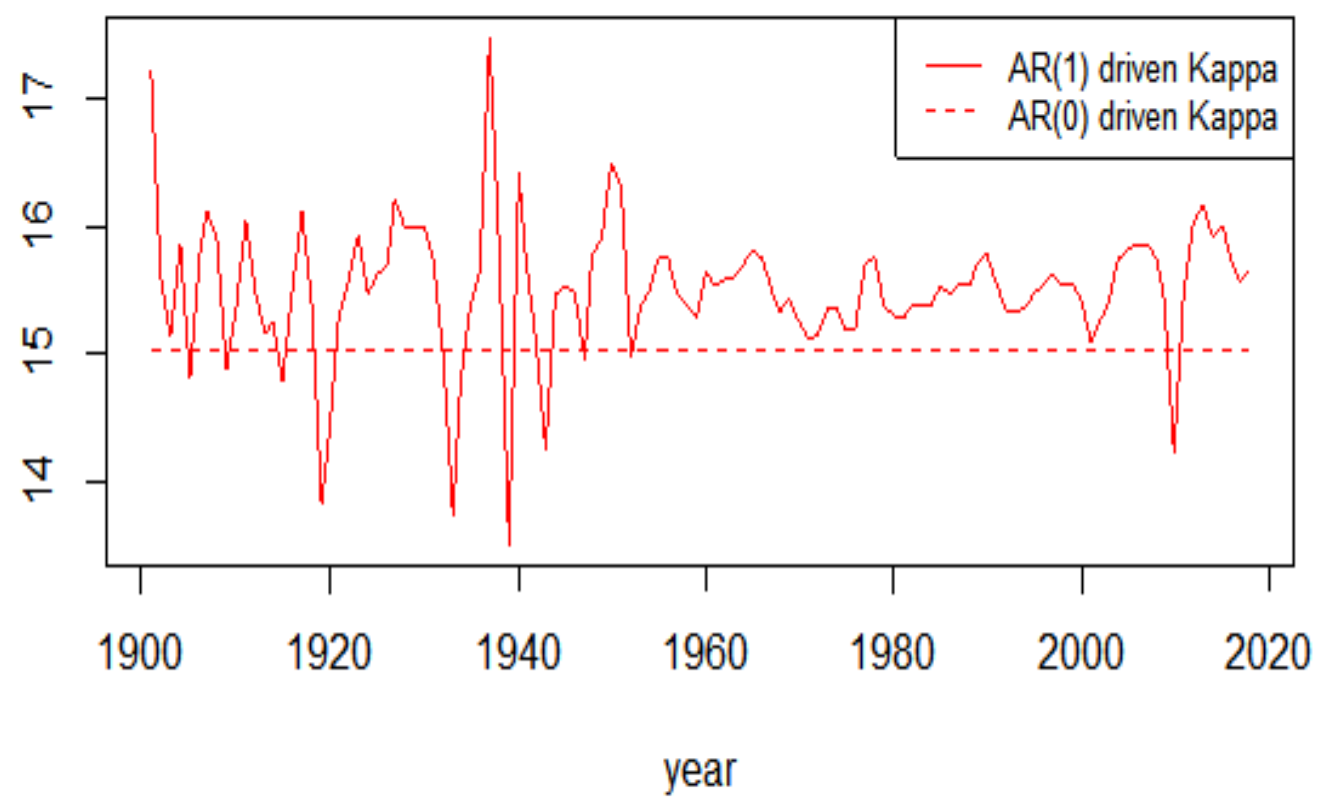

(b) 
Figure 2.5: Implied AR(1) Model Results

Implied AR(|) Model Results (DJA)

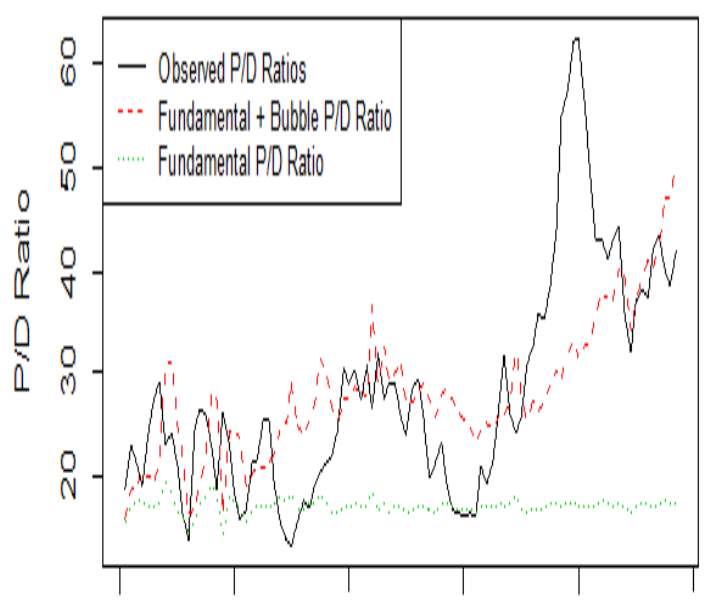

$\begin{array}{lllll}1920 & 1940 \quad 1960 & 1990 \quad 2000 & 2020\end{array}$

(a)

Implied AR(1) Model Results (S\&P 500)

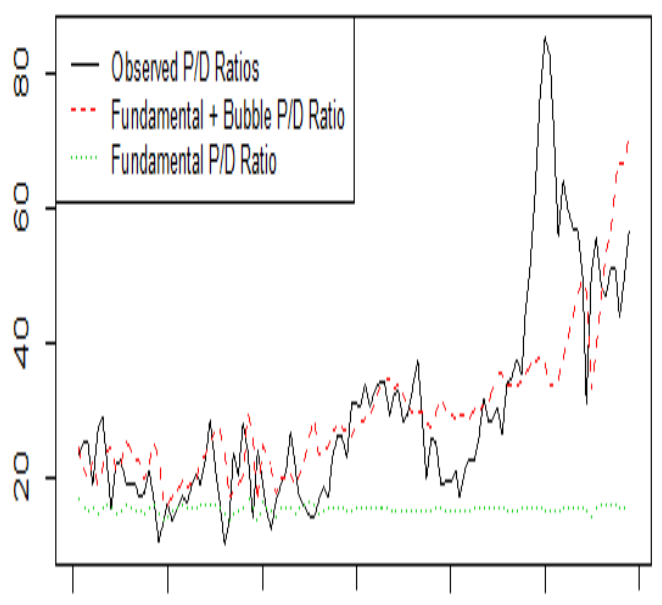

$\begin{array}{llllll}1900 & 1920 \quad 1940 \quad 1960 & 1990 & 2000 & 2020\end{array}$
Implied AR(|) Model Results (DJNA)

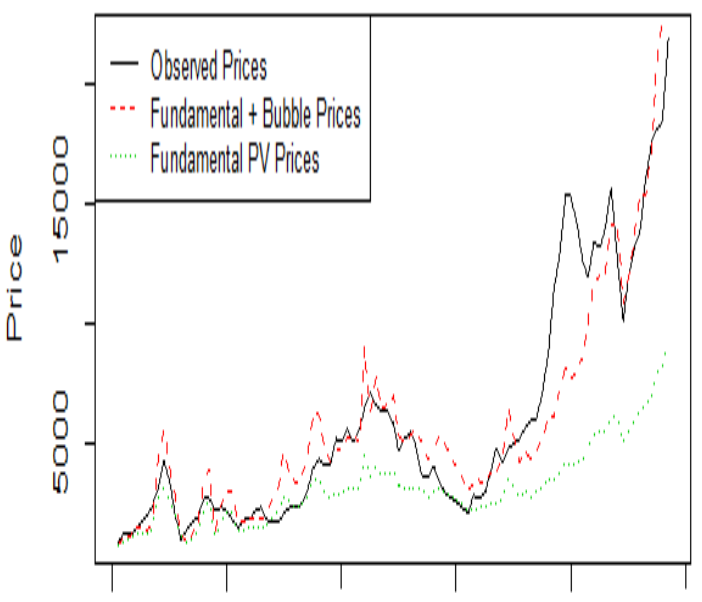

$\begin{array}{llllll}1920 & 1940 \quad 1960 & 1980 & 2000 & 2020\end{array}$

(b)

Implied AR(1) Model Results (SSP 500)

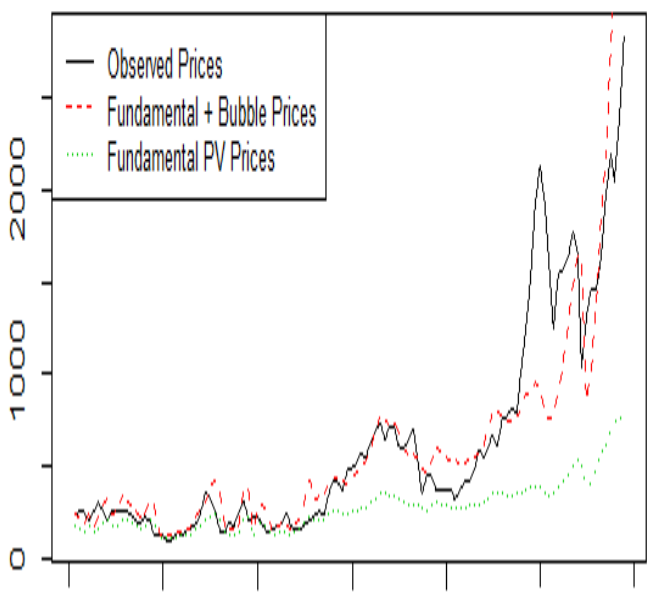

$\begin{array}{llllll}1900 & 1920 \quad 1940 \quad 1960 & 1980 & 2000 & 2020\end{array}$ 
Figure 2.6: Implied $A R(0)$ Model Results

Implied AR(O) Model Results (DJIA)

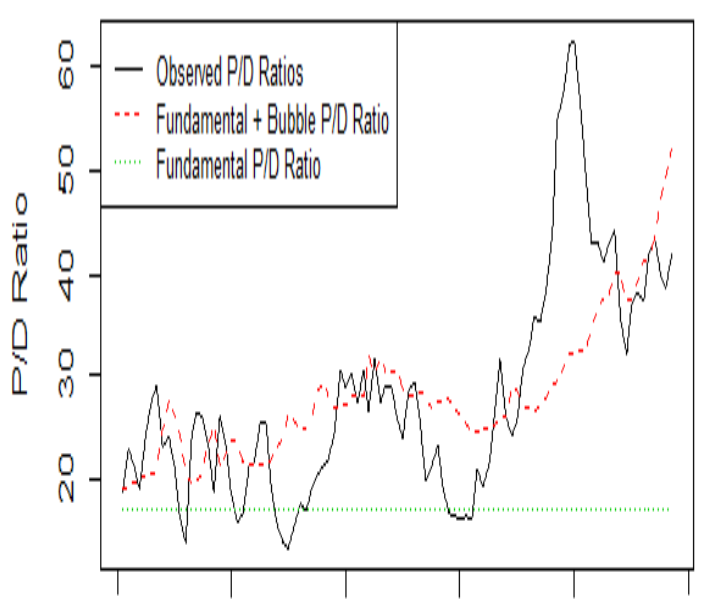

$\begin{array}{llllll}1920 & 1940 \quad 1960 & 1980 & 2000 & 2020\end{array}$

(a)

Implied AR(O) Model Results (S\&P 500)

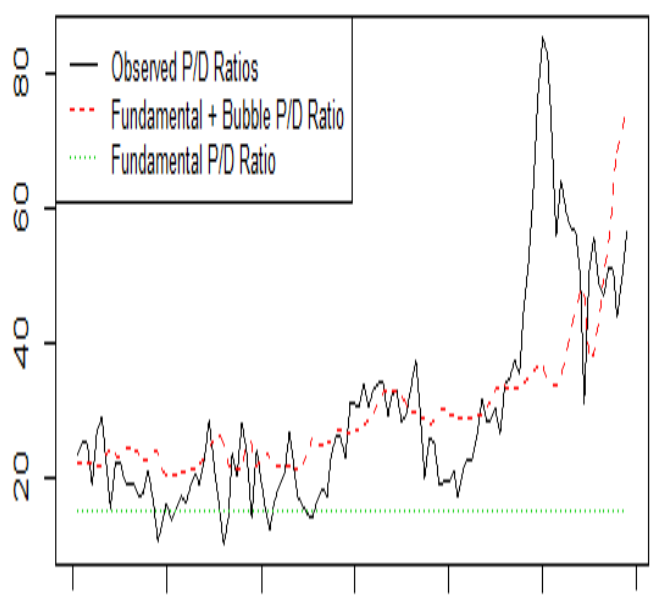

$\begin{array}{lllllll}1900 & 1920 & 1940 & 1960 & 1980 & 2000 & 2020\end{array}$
Implied AR(O) Model Results (DJAA)

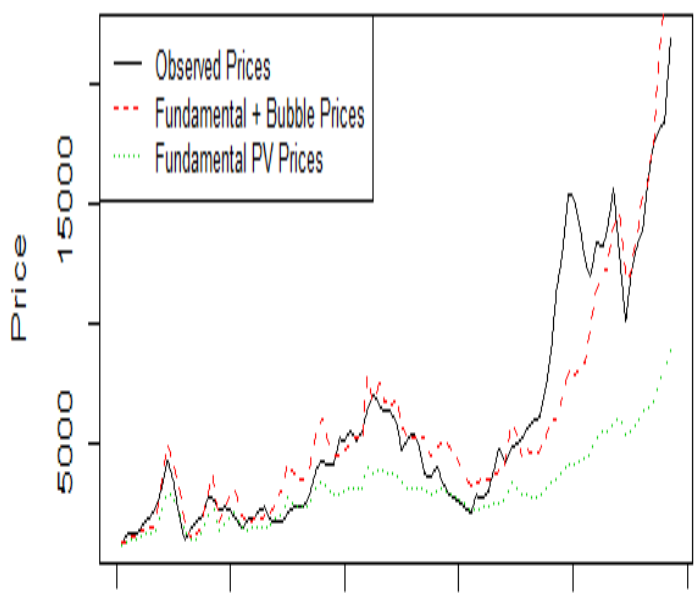

$\begin{array}{llllll}1920 & 1940 \quad 1990 & 1980 & 2000 & 2020\end{array}$

(b)

Implied AR(O) Model Results (S\&P 500)

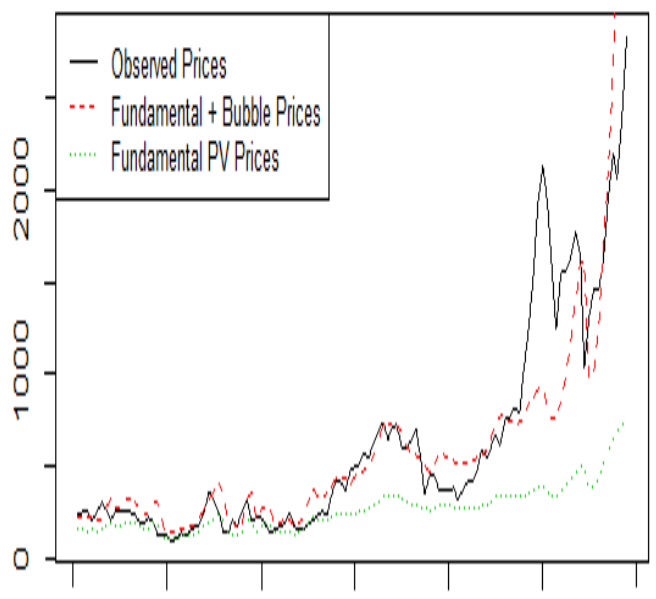

$\begin{array}{lllllll}1900 & 1920 & 1940 & 1960 & 1980 & 2000 & 2020\end{array}$ 
Figure 2.7: Comparison of fundamental components [Implied AR(1) vs. AR(0)]
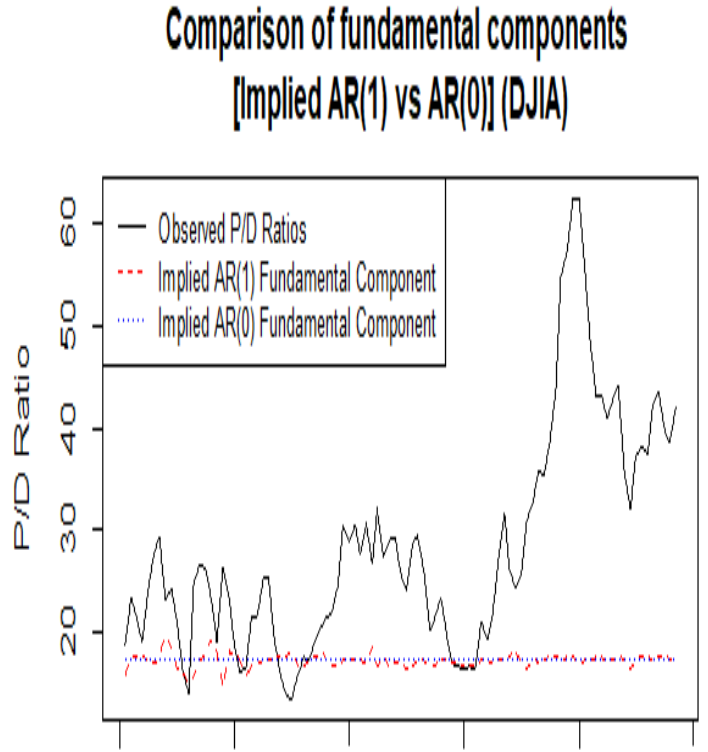

$1920 \quad 1940 \quad 19900 \quad 1980 \quad 2000 \quad 2020$

(a)

\section{Comparison of fundamental components |Implied ARII) vs AR(O) (SQP 500)}

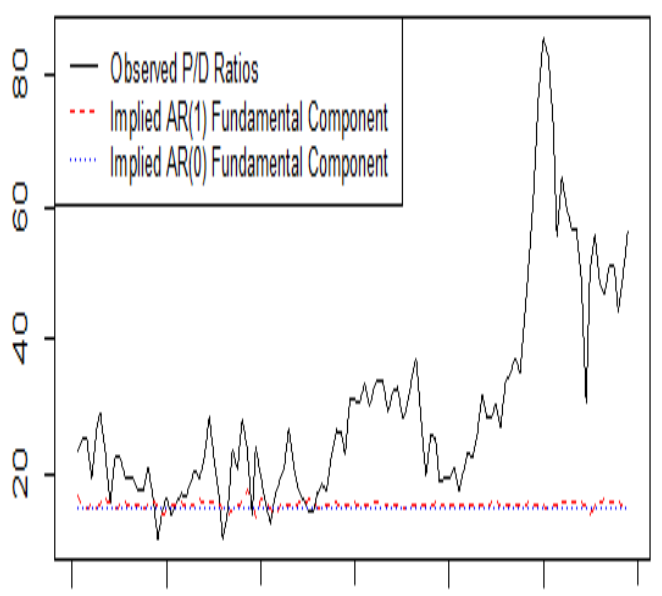

$\begin{array}{llllll}1900 & 1920 \quad 1940 \quad 1960 & 1980 & 2000 & 2020\end{array}$

\section{Comparison of fundamental components \\ Implied AR(1) vs AR(O)||(DJAA)}

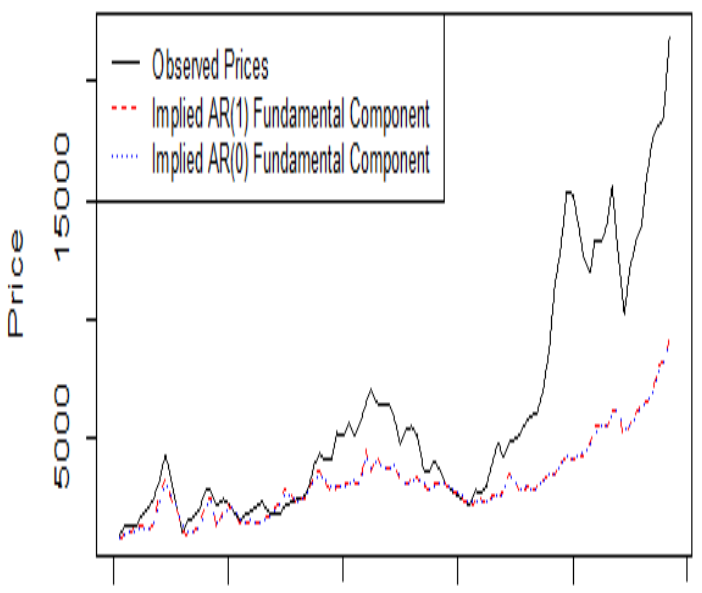

$1920 \quad 1940 \quad 1990 \quad 1980 \quad 2000 \quad 2020$

(b)
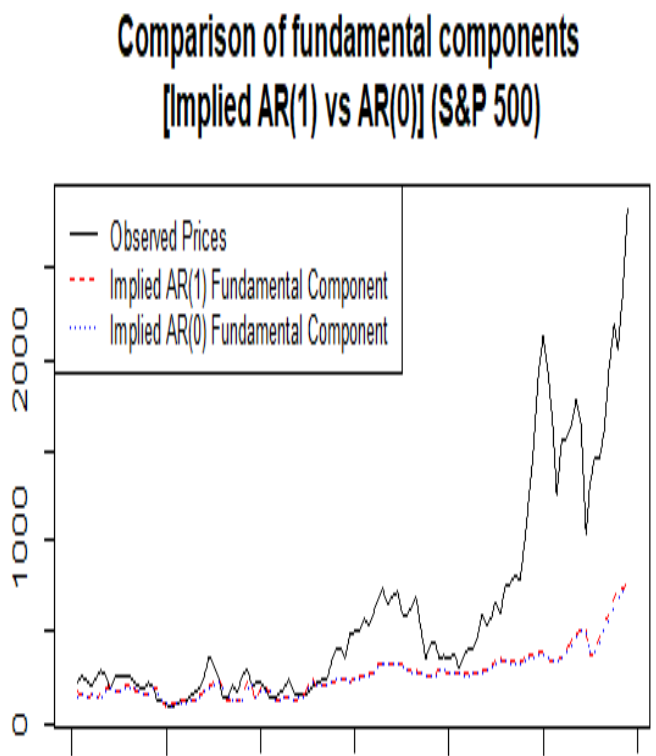

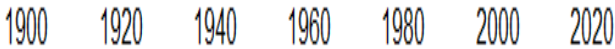


Figure 2.8: Comparison of both fundamental and bubble components [Implied AR(1) vs. $\mathrm{AR}(0)]$
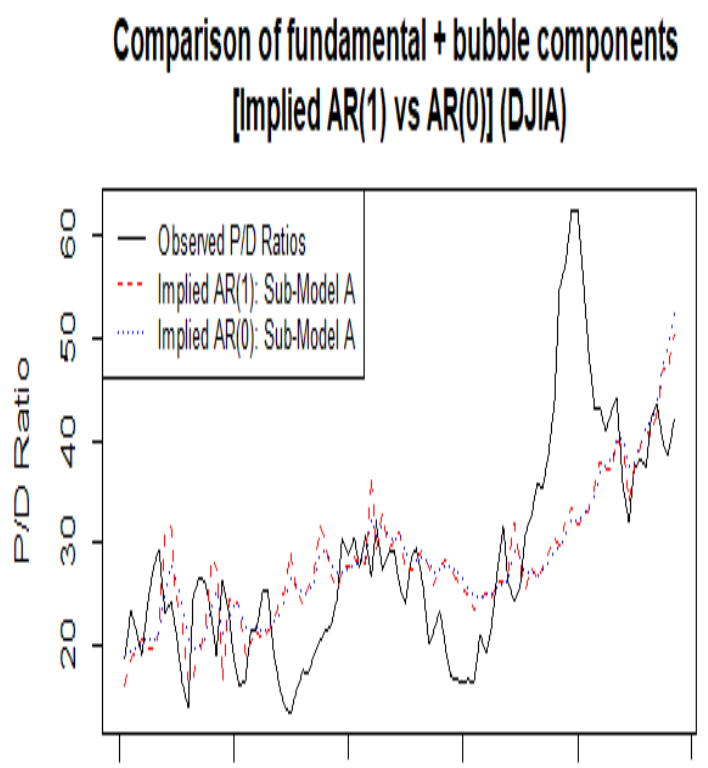

$1920 \quad 1940 \quad 1960 \quad 1980 \quad 2000 \quad 2020$

(a)
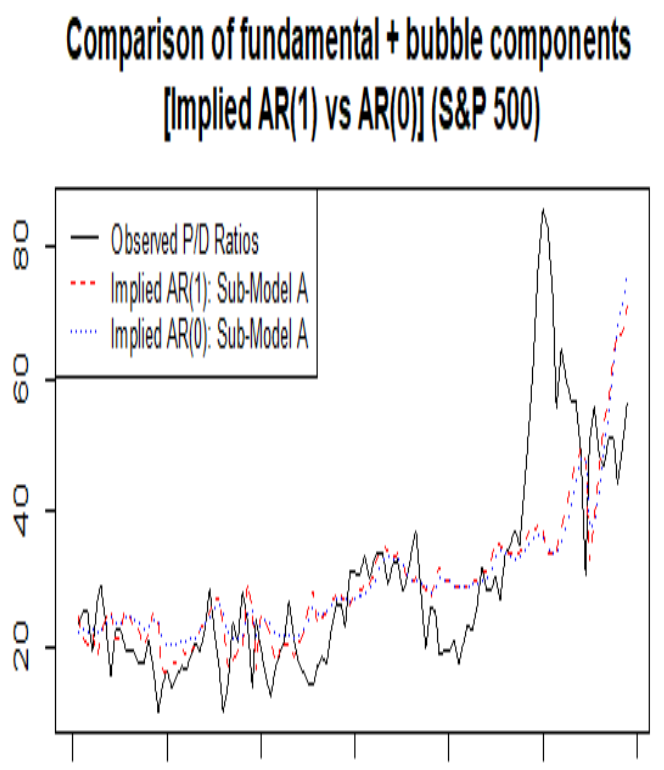

$1900 \quad 1920 \quad 1940 \quad 1960 \quad 1980 \quad 2000 \quad 2020$
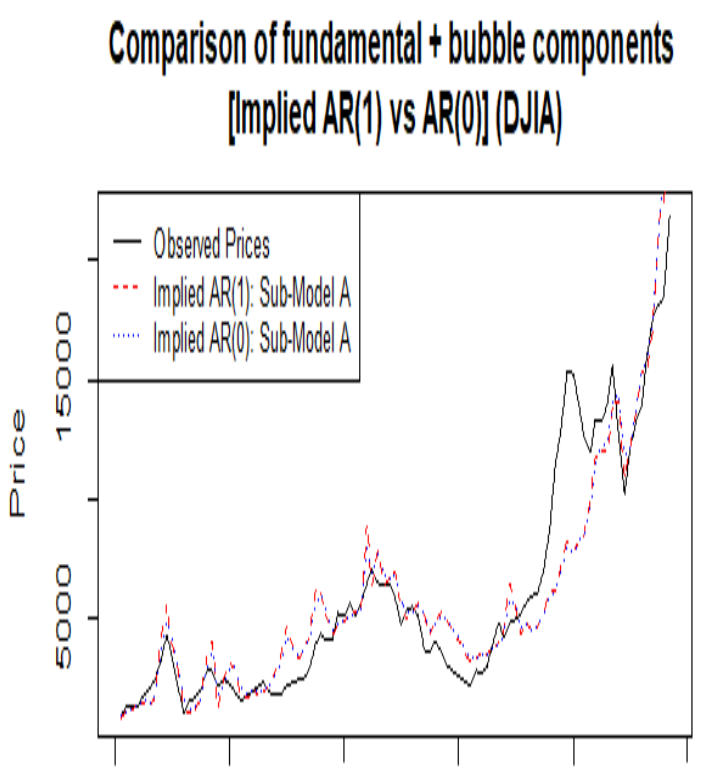

$1920 \quad 1940 \quad 1960 \quad 1980 \quad 2000 \quad 2020$

(b)

Comparison of fundamental + tbubble components

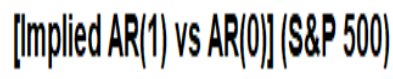

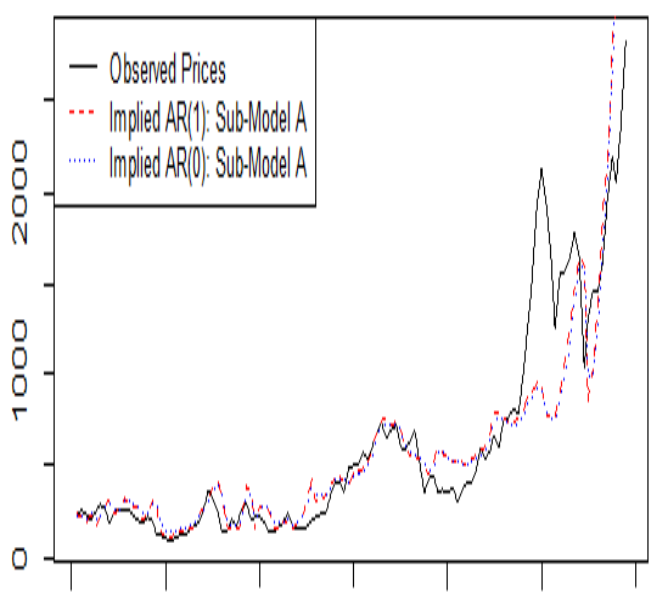

$1900 \quad 1920 \quad 1940 \quad 1960 \quad 1980 \quad 2000 \quad 2020$ 
Figure 2.9: Free AR(1) Model Results

Free AR(1) Model Results (DJIA)

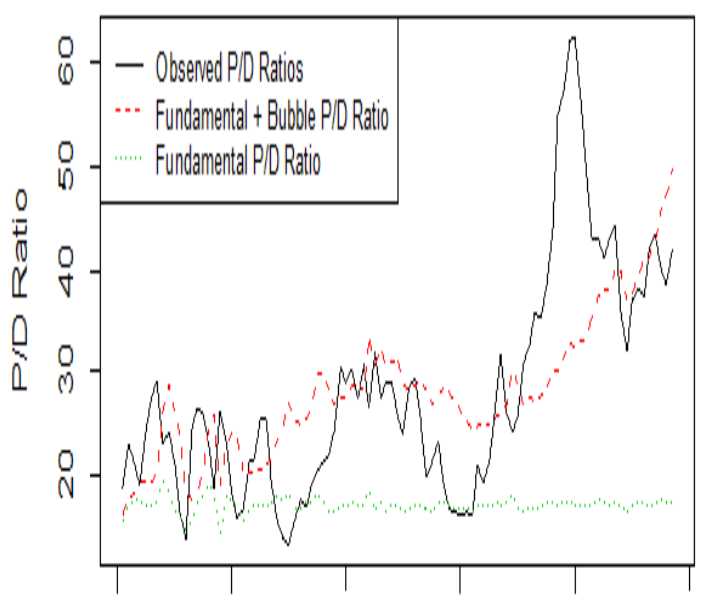

$\begin{array}{lllll}1920 & 1940 \quad 1960 \quad 1980 & 2000 & 2020\end{array}$

(a)

Free ARI|) ModeleResults (SSP 500)

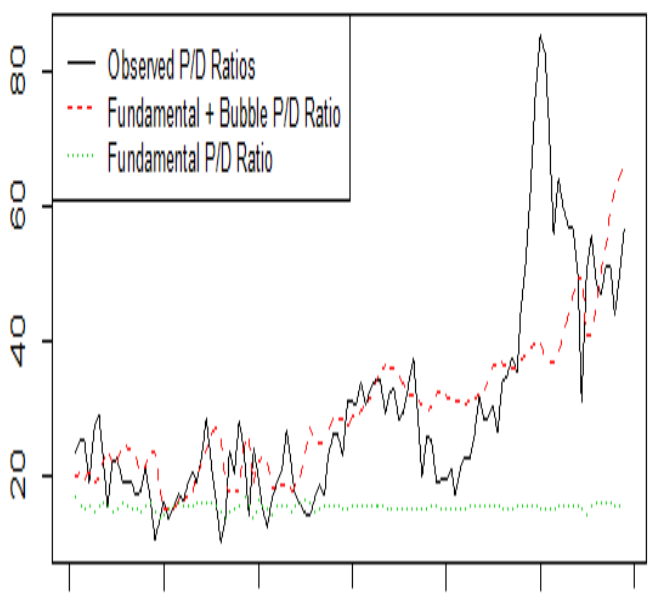

$\begin{array}{lllllll}1900 & 1920 & 1940 & 1960 & 1980 & 2000 & 2020\end{array}$
Free AR(1) Model Results (DJIA)

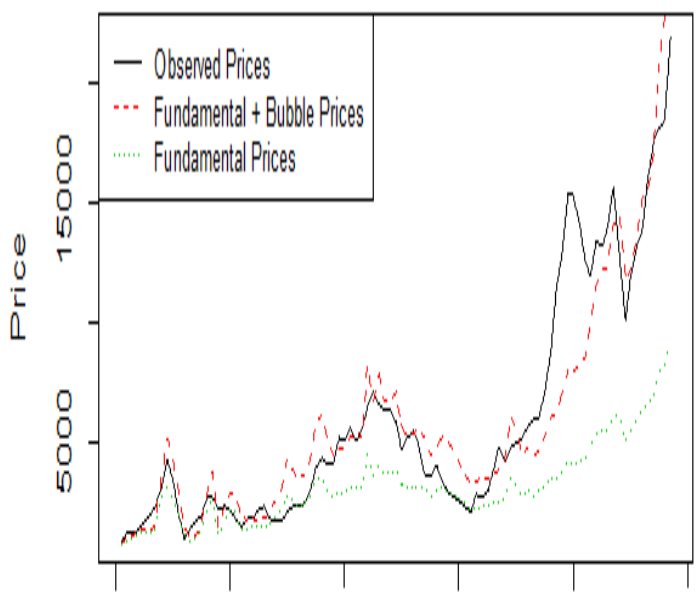

$\begin{array}{llllll}1920 & 1940 & 1960 & 1980 & 2000 & 2020\end{array}$

(b)

Free AR(1) Model Results (SLP 500)

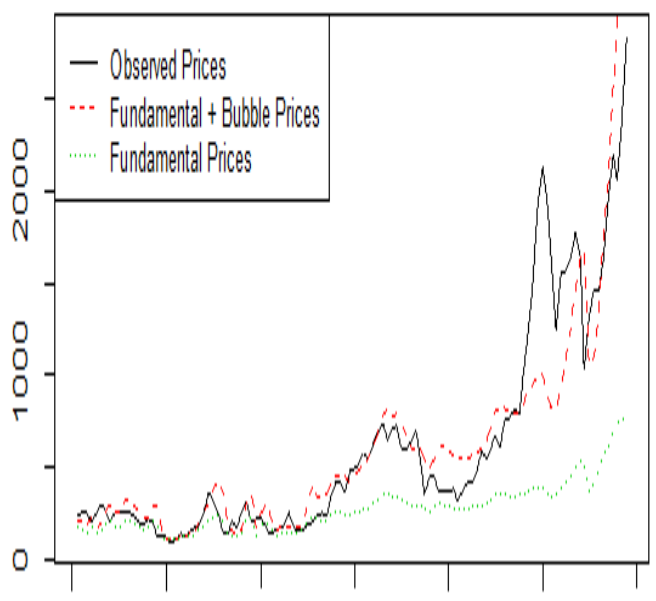

$\begin{array}{lllllll}1900 & 1920 & 1940 & 1960 & 1980 & 2000 & 2020\end{array}$ 
Figure 2.10: Free AR(0) Model Results

Free ARPO) Model Results (DJA)

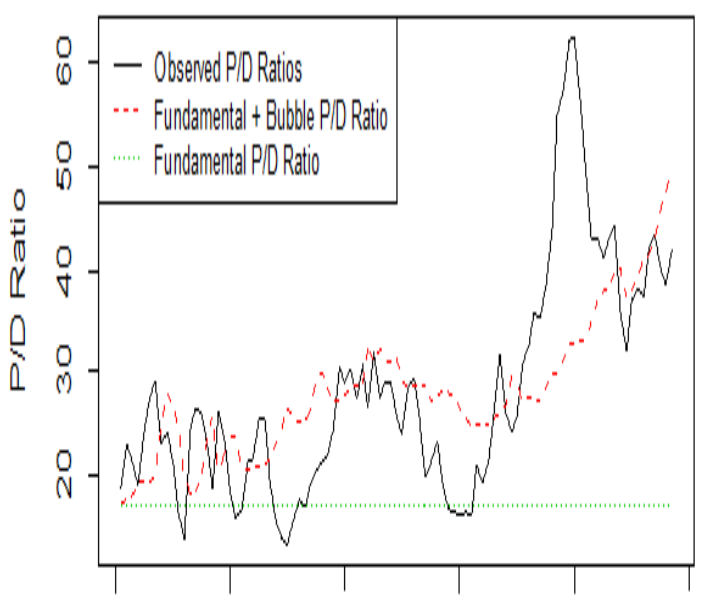

$\begin{array}{lllll}1920 & 1940 \quad 1960 & 1980 & 2000 & 2020\end{array}$

(a)

Free AR(O) Model Results (S\&P 500)

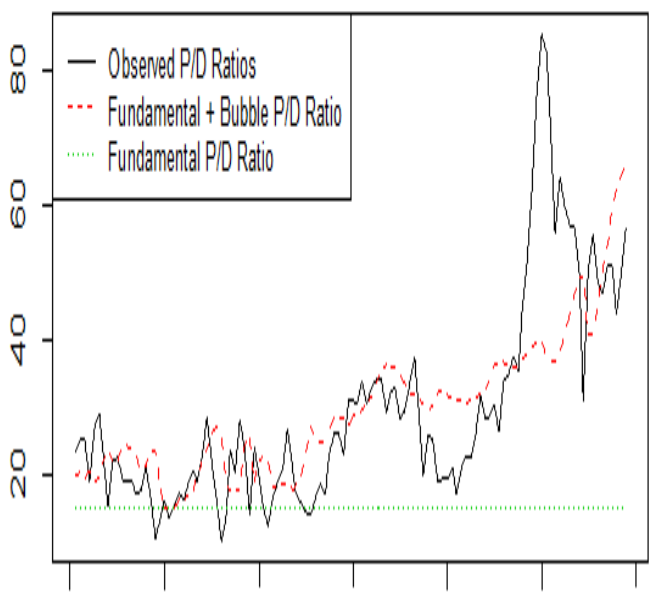

$\begin{array}{llllll}1900 & 1920 \quad 1940 \quad 1960 & 1990 & 2000 & 2020\end{array}$
Free ARR(O) Model Results (DJAA)

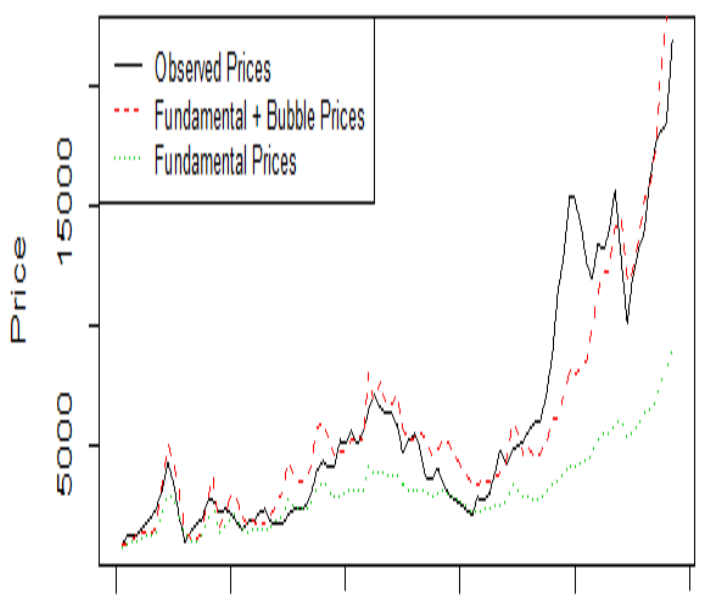

$\begin{array}{llllll}1920 & 1940 \quad 1960 & 1980 & 2000 & 2020\end{array}$

(b)

Free AR(O) Model Results (S\&P 500)

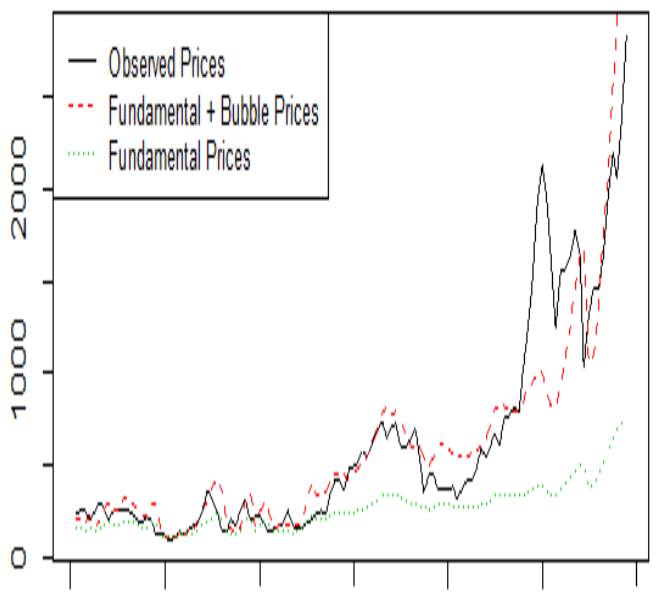

$\begin{array}{lllllll}1900 & 1920 & 1940 & 1960 & 1990 & 2000 & 2020\end{array}$ 
Figure 2.11: Comparison of both fundamental and bubble components [Free AR(1) vs. $\mathrm{AR}(0)]$
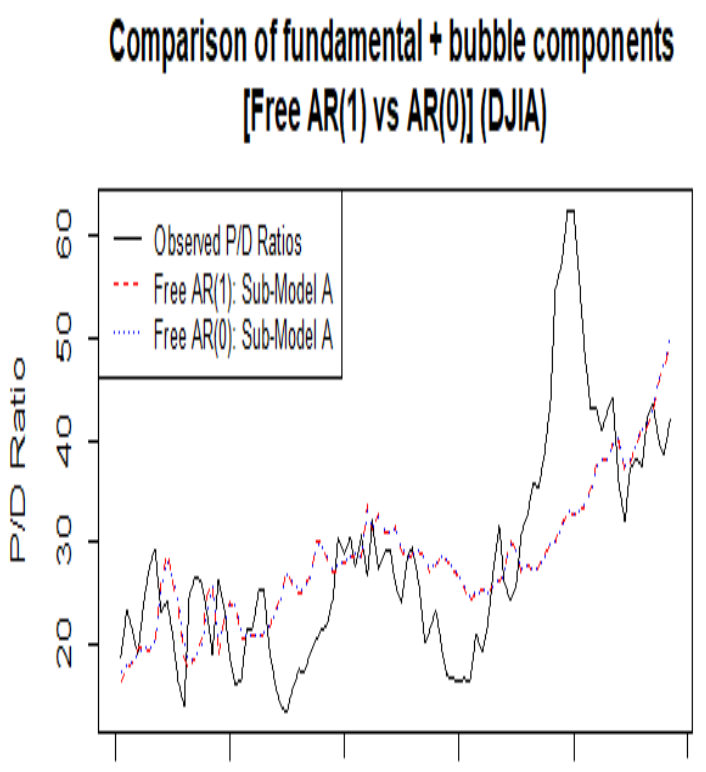

$1920 \quad 1940 \quad 1960 \quad 1980 \quad 2000 \quad 2020$

(a)
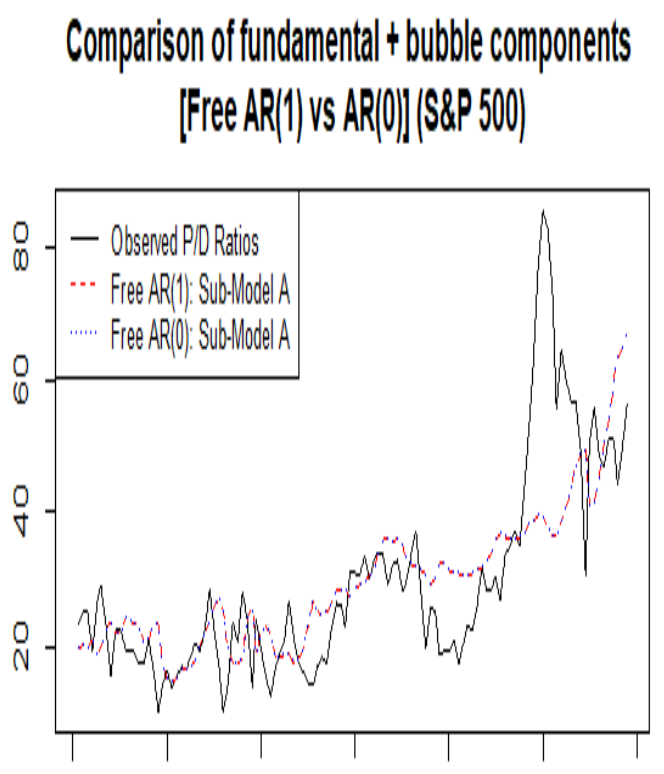

$1900 \quad 1920 \quad 1940 \quad 1960 \quad 1980 \quad 2000 \quad 2020$
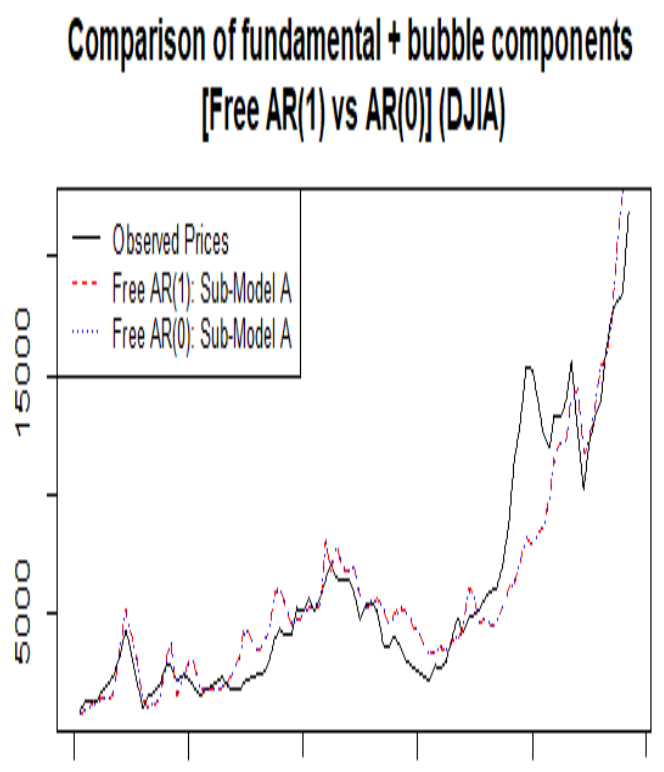

$1920 \quad 1940 \quad 1960 \quad 1990 \quad 2000 \quad 2020$

(b)

Comparison of fundamental + bubble components

|Free AR(1) vs AR(O)| (S\&P 500)

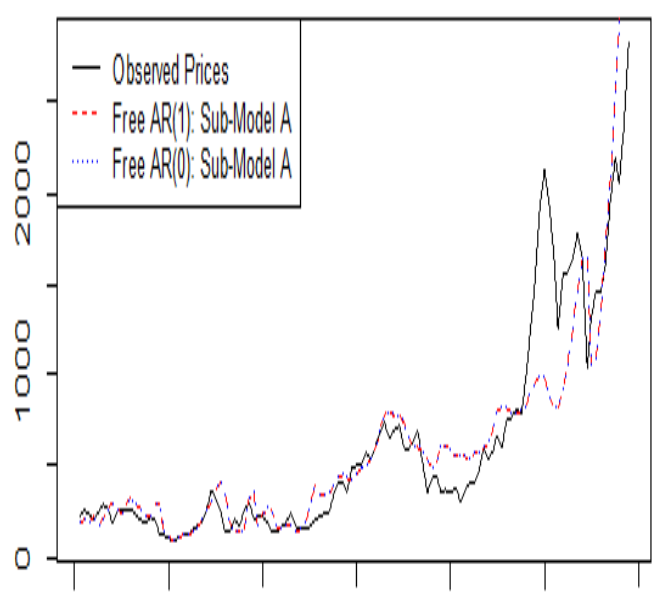

$\begin{array}{llllll}1900 & 1920 & 1940 & 1960 & 1980 \quad 2000 & 2020\end{array}$ 
Figure 2.12: Comparison of both fundamental and bubble components [Implied vs. Free $\mathrm{AR}(1)]$
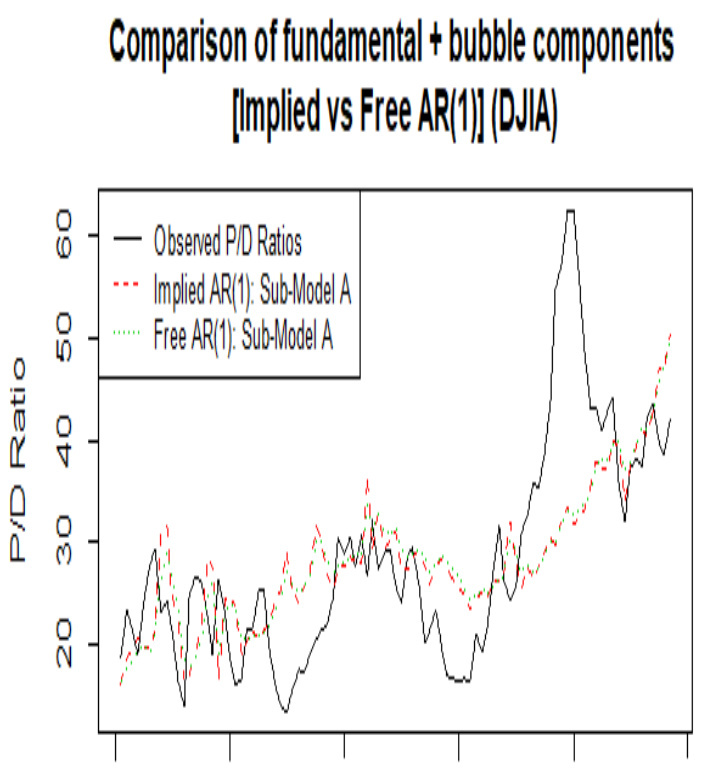

$\begin{array}{lllll}1920 & 1940 \quad 19900 \quad 1980 & 2000 & 2020\end{array}$

(a)
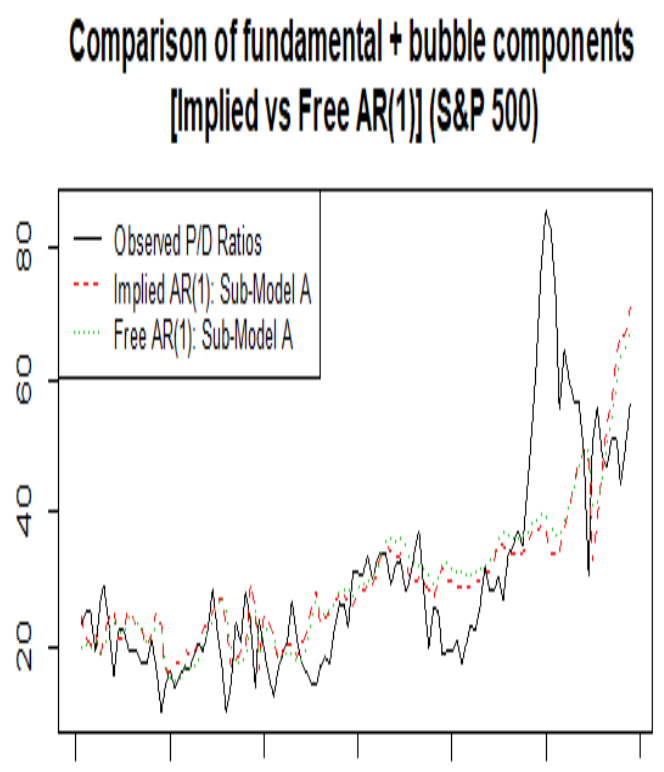

$1900 \quad 1920 \quad 1940 \quad 1960 \quad 1980 \quad 2000 \quad 2000$
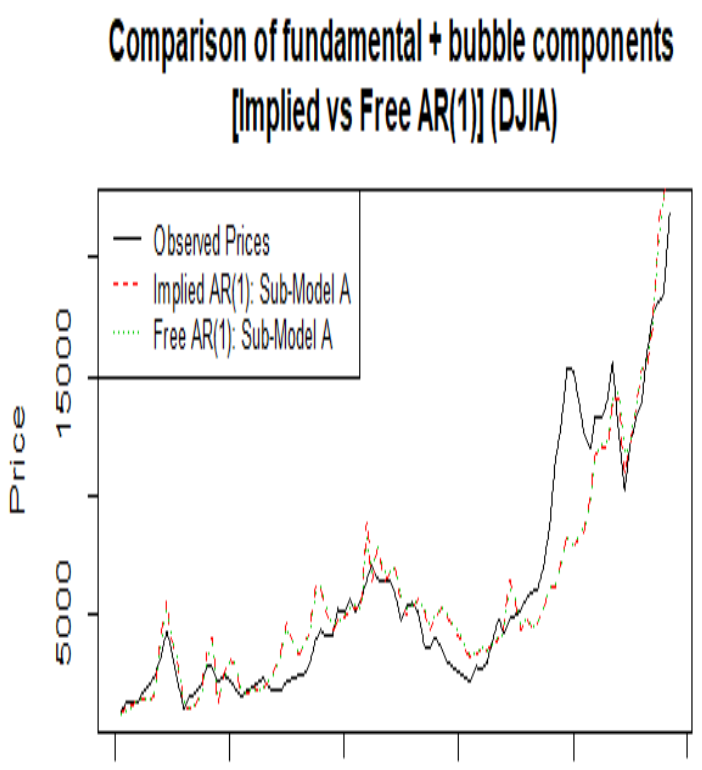

$1920 \quad 1940 \quad 1960 \quad 1980 \quad 2000 \quad 2020$

(b)

Comparison of fundamental + bubble components

|lmplied vs Free AR(1)|)(SEP 500)

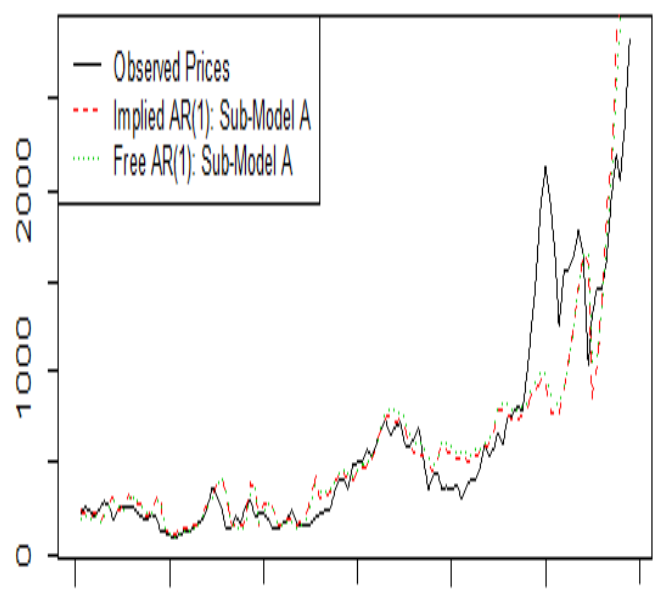

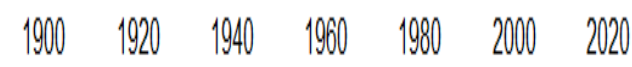


Figure 2.13: Comparison of both fundamental and bubble components [Implied vs. Free $\mathrm{AR}(0)]$
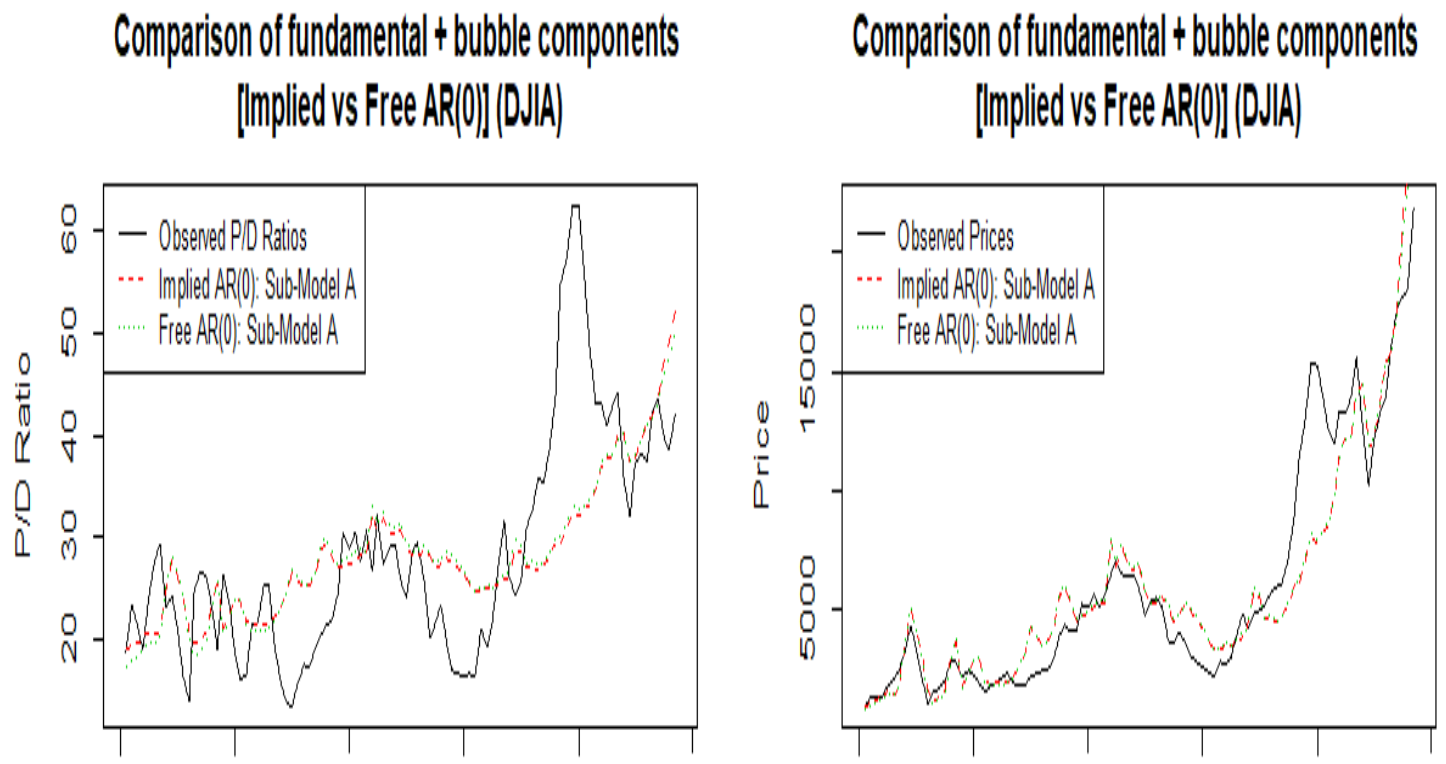

$\begin{array}{llllll}1920 & 1940 \quad 1960 & 1980 & 2000 & 2020\end{array}$

(a)

(b)

Comparison of fundamental \& bubble components

Implied vs Free AR(O) (SSP 500)
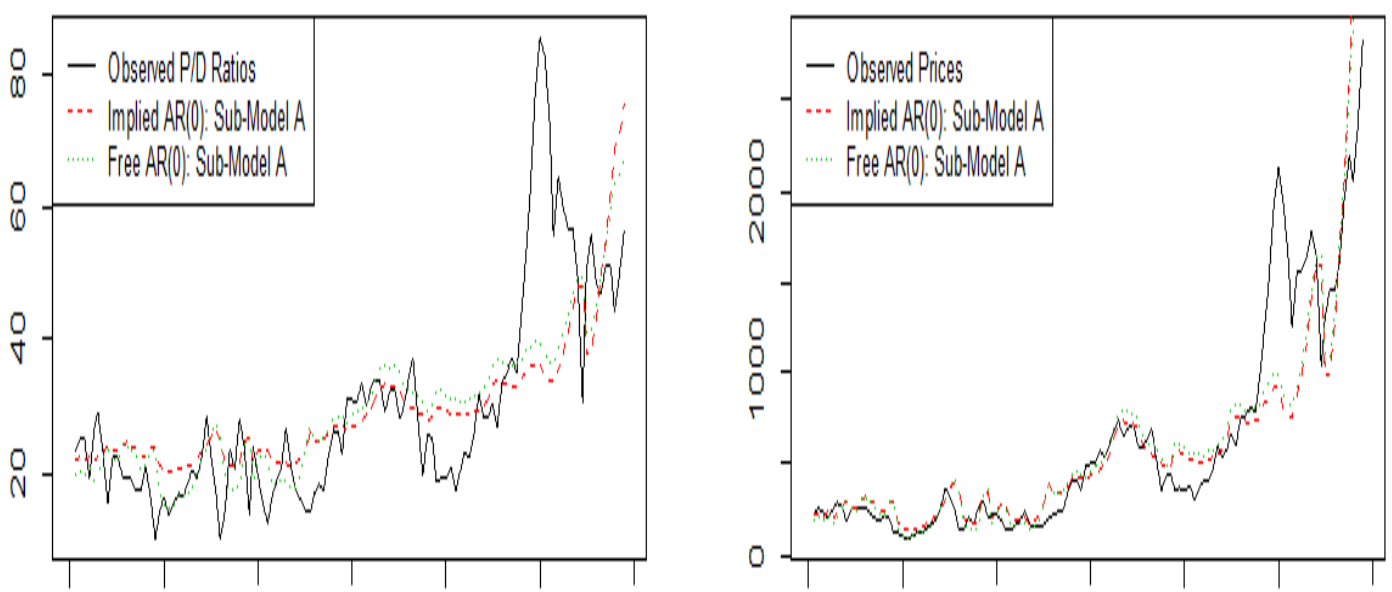

$1900 \quad 1920 \quad 1940 \quad 1960 \quad 1980 \quad 2000 \quad 2000$

$1900 \quad 1920 \quad 1940 \quad 1960 \quad 1980 \quad 2000 \quad 2020$ 


\subsection{Appendix}

\subsubsection{Appendix A}

\section{Derivation of the Fundamental Stock Price}

In this appendix we derive the expression for the fundamental stock price $P_{t}^{p v}$ given in Equation (2.7). From Equation (2.2), we have:

$$
P_{t}^{p v}=\sum_{s=t}^{\infty} \exp \{-r(s-t+1)\} E_{t}\left(D_{s}\right)
$$

For $\mathrm{s}=\mathrm{t}+1, \mathrm{t}+2, \ldots$

$$
\mathrm{D}_{\mathrm{s}}=\mathrm{D}_{\mathrm{t}+\mathrm{s}-\mathrm{t}}=\exp \left\{\mathrm{d}_{\mathrm{t}+\mathrm{s}-\mathrm{t}}\right\}=\exp \left\{\mathrm{x}_{\mathrm{t}+\mathrm{s}-\mathrm{t}}+\mathrm{x}_{\mathrm{t}+\mathrm{s}-\mathrm{t}-1}+\mathrm{x}_{\mathrm{t}+\mathrm{s}-\mathrm{t} \ldots}+\mathrm{x}_{\mathrm{t}+1}+\mathrm{d}_{\mathrm{t}}\right\} .
$$

Using Equation (2.15) from Burnside (1998),

$$
\begin{gathered}
\sum_{j=1}^{s-t} x_{t+j}=(s-t) \mu+\frac{\rho}{1-\rho}\left\{1-\rho^{s-t}\right\}\left(x_{t}-\mu\right)+ \\
(1-\rho)^{-1}\left[\left(1-\rho^{s-t}\right) \xi_{t+1}+\left(1-\rho^{s-t-1}\right) \xi_{t+2}+\ldots+(1-\rho) \xi_{t+s-t}\right] .
\end{gathered}
$$

Therefore,

$$
\mathrm{E}_{\mathrm{t}}\left(\mathrm{D}_{\mathrm{s}}\right)=\mathrm{D}_{\mathrm{t}} \exp \left\{\begin{array}{c}
(\mathrm{s}-\mathrm{t}) \mu+\frac{\rho}{1-\rho}\left\{1-\rho^{\mathrm{s}-\mathrm{t}}\right\}\left(\mathrm{x}_{\mathrm{t}}-\mu\right)+ \\
\frac{\sigma^{2}}{2(1-\rho)^{2}}\left[\left(1-\rho^{\mathrm{s}-\mathrm{t}}\right)^{2}+\left(1-\rho^{\mathrm{s}-\mathrm{t}-1}\right)^{2}+\ldots+(1-\rho)^{2}\right]
\end{array}\right\} .
$$


This simplifies to:

$$
\mathrm{E}_{\mathrm{t}}\left(\mathrm{D}_{\mathrm{s}}\right)=\mathrm{D}_{\mathrm{t}} \exp \left\{\begin{array}{c}
\frac{\rho}{1-\rho}\left\{1-\rho^{\mathrm{s}-\mathrm{t}}\right\}\left(\mathrm{x}_{\mathrm{t}}-\mu\right)+ \\
(\mathrm{s}-\mathrm{t}) \mu+\frac{\sigma^{2}}{2(1-\rho)^{2}}\left[(\mathrm{~s}-\mathrm{t})-2 \frac{\rho}{1-\rho}\left(1-\rho^{\mathrm{s}-\mathrm{t}}\right)+\rho^{2} \frac{1-\rho^{2(\mathrm{~s}-\mathrm{t})}}{1-\rho^{2}}\right]
\end{array}\right\}
$$

which can be expressed as:

$$
\mathrm{E}_{\mathrm{t}}\left(\mathrm{D}_{\mathrm{s}}\right)=\mathrm{D}_{\mathrm{t}} \exp \left\{\mathrm{b}_{\mathrm{s}-\mathrm{t}}\left(\mathrm{x}_{\mathrm{t}}-\mu\right)+\mathrm{a}_{\mathrm{s}-\mathrm{t}}\right\}
$$

with

$$
a_{s-t}=(s-t) \mu+\frac{\sigma^{2}}{2(1-\rho)^{2}}\left[(s-t)-2 \frac{\rho}{1-\rho}\left(1-\rho^{s-t}\right)+\rho^{2} \frac{1-\rho^{2(s-t)}}{1-\rho^{2}}\right]
$$

and

$$
\mathrm{b}_{\mathrm{s}-\mathrm{t}}=\frac{\rho}{1-\rho}\left\{1-\rho^{\mathrm{s}-\mathrm{t}}\right\}
$$

Equation (2.29) can be expressed as $P_{t}^{p v}=\exp \{-r\} D_{t}+\sum_{s=t+1}^{\infty} \exp \{-r(s-t+$ 1) $\} E_{t}\left(D_{s}\right)$. Substituting for $E_{t}\left(D_{s}\right)$ yields:

$$
\mathrm{P}_{\mathrm{t}}^{\mathrm{pv}}=\exp \{-\mathrm{r}\} \mathrm{D}_{\mathrm{t}}+\mathrm{D}_{\mathrm{t}} \sum_{\mathrm{s}=\mathrm{t}+1}^{\infty} \exp \left\{-\mathrm{r}(\mathrm{s}-\mathrm{t}+1)+\mathrm{b}_{\mathrm{s}-\mathrm{t}}\left(\mathrm{x}_{\mathrm{t}}-\mu\right)+\mathrm{a}_{\mathrm{s}-\mathrm{t}}\right\}
$$

which can be rewritten as:

$$
\mathrm{P}_{\mathrm{t}}^{\mathrm{pv}}=\mathrm{D}_{\mathrm{t}} \sum_{\mathrm{s}=\mathrm{t}}^{\infty} \exp \left\{-\mathrm{r}(\mathrm{s}-\mathrm{t}+1)+\mathrm{b}_{\mathrm{s}-\mathrm{t}}\left(\mathrm{x}_{\mathrm{t}}-\mu\right)+\mathrm{a}_{\mathrm{s}-\mathrm{t}}\right\}
$$




\subsubsection{Appendix B}

\section{Proof of Theorem 1: Convergence of the Price-Dividend Ratio}

From Equation (2.7),

$$
\mathrm{P}_{\mathrm{t}}^{\mathrm{pv}}=\mathrm{D}_{\mathrm{t}} \sum_{\mathrm{s}=\mathrm{t}}^{\infty} \exp \left\{-\mathrm{r}(\mathrm{s}-\mathrm{t}+1)+\mathrm{b}_{\mathrm{s}-\mathrm{t}}\left(\mathrm{x}_{\mathrm{t}}-\mu\right)+\mathrm{a}_{\mathrm{s}-\mathrm{t}}\right\}
$$

where

$$
a_{s-t}=(s-t) \mu+\frac{\sigma^{2}}{2(1-\rho)^{2}}\left[(s-t)-2 \frac{\rho}{1-\rho}\left(1-\rho^{s-t}\right)+\rho^{2} \frac{1-\rho^{2(s-t)}}{1-\rho^{2}}\right]
$$

and

$$
\begin{gathered}
\mathrm{b}_{\mathrm{s}-\mathrm{t}}=\frac{\rho}{1-\rho}\left\{1-\rho^{\mathrm{s}-\mathrm{t}}\right\} \\
\text { Denoting } \mathrm{i}=\mathrm{s}-\mathrm{t}, \mathrm{v}_{\mathrm{t}}=\frac{\mathrm{P}_{\mathrm{t}}^{\mathrm{pv}}}{\mathrm{D}_{\mathrm{t}}}=\sum_{\mathrm{i}=0}^{\infty} \exp \{-\mathrm{r}(\mathrm{i}+1)\} \exp \left\{\mathrm{a}_{\mathrm{i}}+\mathrm{b}_{\mathrm{i}}\left(\mathrm{x}_{\mathrm{t}}-\mu\right)\right\}
\end{gathered}
$$

where

$$
\mathrm{b}_{\mathrm{i}}=\frac{\rho}{1-\rho}\left\{1-\rho^{\mathrm{i}}\right\} \text { and } a_{\mathrm{i}}=\mathrm{i} \mu+\frac{\sigma^{2}}{2(1-\rho)^{2}}\left[\mathrm{i}-2 \frac{\rho}{1-\rho}\left(1-\rho^{\mathrm{i}}\right)+\rho^{2} \frac{1-\rho^{2 \mathrm{i}}}{1-\rho^{2}}\right]
$$

Let

$$
\mathrm{v}_{\mathrm{t}} \equiv \sum_{i=0}^{\infty} z_{i}
$$

Then

$$
\frac{\mathrm{z}_{\mathrm{i}+1}}{\mathrm{z}_{\mathrm{i}}}=\frac{\exp \{-\mathrm{r}(\mathrm{i}+1+1)\} \exp \left\{a_{\mathrm{i}+1}+\mathrm{b}_{\mathrm{i}+1}\left(\mathrm{x}_{\mathrm{t}}-\mu\right)\right\}}{\exp \{-\mathrm{r}(\mathrm{i}+1)\} \exp \left\{\mathrm{a}_{\mathrm{i}}+\mathrm{b}_{\mathrm{i}}\left(\mathrm{x}_{\mathrm{t}}-\mu\right)\right\}}
$$




$$
\begin{gathered}
=\exp \{-\mathrm{r}\} \exp \left\{\left(\mathrm{a}_{i+1}-\mathrm{a}_{\mathrm{i}}\right)+\left(\mathrm{b}_{\mathrm{i}+1}-\mathrm{b}_{\mathrm{i}}\right)\left(\mathrm{x}_{\mathrm{t}}-\mu\right)\right\} \\
\mathrm{b}_{\mathrm{i}+1}-\mathrm{b}_{\mathrm{i}}=\frac{\rho}{1-\rho}\left\{1-\rho^{\mathrm{i}+1}-1+\rho^{\mathrm{i}}\right\}=\rho^{\mathrm{i}+1} \\
a_{i+1}-a_{i}=\mu+\frac{\sigma^{2}}{2(1-\rho)^{2}}\left[1-2 \frac{\rho}{1-\rho}\left(1-\rho^{i+1}-1+\rho^{i}\right)+\frac{\rho^{2}}{1-\rho^{2}}\left\{1-\rho^{2(i+1)}-1+\rho^{2 i}\right\}\right] \\
=\mu+\frac{\sigma^{2}}{2(1-\rho)^{2}}\left[1+\rho^{i+1}\left\{\rho^{i+1}-2\right\}\right] .
\end{gathered}
$$

Therefore,

$$
\frac{\mathrm{Z}_{\mathrm{i}+1}}{\mathrm{Z}_{\mathrm{i}}}=\exp \left\{-\mathrm{r}+\mu+\frac{\sigma^{2}}{2\left(1-\rho^{2}\right)}\left[1+\rho^{\mathrm{i}+1}\left(\rho^{\mathrm{i}+1}-2\right)\right]+\rho^{\mathrm{i}+1}\left(\mathrm{x}_{\mathrm{t}}-\mu\right)\right\} .
$$

Now $\frac{z_{i+1}}{z_{i}}=\left|\frac{z_{i+1}}{z_{i}}\right|$. With $|\rho<1|, \lim _{i \rightarrow \infty}\left|\frac{z_{i+1}}{z_{i}}\right|=\exp \left\{-r+\mu+\frac{\sigma^{2}}{2\left(1-\rho^{2}\right)}\right\}$.

Therefore, from Burnside's (1998) proof of Theorem 1, $\sum_{i=0}^{\infty} z_{i}$ converges if

$$
\mathrm{R} \equiv \exp \left\{-\mathrm{r}+\mu+\frac{\sigma^{2}}{2\left(1-\rho^{2}\right)}\right\}<1
$$

\subsubsection{Appendix C}

\section{Derivation of Equation (2.11): Mean of the Price-Dividend Ratio}

From Equation (2.7), we have:

$$
\mathrm{v}_{\mathrm{t}} \equiv \frac{\mathrm{P}_{\mathrm{t}}^{\mathrm{pv}}}{\mathrm{D}_{\mathrm{t}}}=\sum_{\mathrm{i}=0}^{\infty} \exp \{-\mathrm{r}(\mathrm{i}+1)\} \exp \left\{\mathrm{a}_{\mathrm{i}}+\mathrm{b}_{\mathrm{i}}\left(\mathrm{x}_{\mathrm{t}}-\mu\right)\right\}
$$


Therefore,

$$
\mathrm{E}\left(\mathrm{v}_{\mathrm{t}}\right)=\sum_{\mathrm{i}=0}^{\infty} \exp \left\{-\mathrm{r}(\mathrm{i}+1)+\mathrm{a}_{\mathrm{i}}\right\} \mathrm{E}\left[\exp \left\{\mathrm{b}_{\mathrm{i}}\left(\mathrm{x}_{\mathrm{t}}-\mu\right)\right\}\right]
$$

We have from Equation (2.6),

$$
\mathrm{x}_{\mathrm{t}}-\mu=\rho\left(\mathrm{x}_{\mathrm{t}-1}-\mu\right)+\xi_{\mathrm{t}}, \quad|\rho|<1, \quad \xi_{\mathrm{t}} \sim \text { iid } \mathrm{N}\left(0, \sigma^{2}\right)
$$

Therefore, $\mathrm{x}_{\mathrm{t}}-\mu \sim \mathrm{N}\left(0, \frac{\sigma^{2}}{1-\rho^{2}}\right)$ which implies $\mathrm{b}_{\mathrm{i}}\left(\mathrm{x}_{\mathrm{t}}-\mu\right) \sim \mathrm{N}\left(0, \frac{\mathrm{b}_{\mathrm{i}}^{2} \sigma^{2}}{1-\rho^{2}}\right)$. Therefore $\mathrm{E}\left[\exp \left\{\mathrm{b}_{\mathrm{i}}\left(\mathrm{x}_{\mathrm{t}}-\mu\right)\right\}\right]=\exp \left\{\frac{\mathrm{b}_{\mathrm{i}}^{2} \sigma^{2}}{2\left(1-\rho^{2}\right)}\right\}$.

Therefore,

$$
\mathrm{E}\left(\mathrm{v}_{\mathrm{t}}\right)=\sum_{\mathrm{i}=0}^{\infty} \exp \left\{-\mathrm{r}(\mathrm{i}+1)+\mathrm{a}_{\mathrm{i}}+\frac{\mathrm{b}_{\mathrm{i}}^{2} \sigma^{2}}{2\left(1-\rho^{2}\right)}\right\}
$$

\section{Proof of Convergence of Mean of the Price-Dividend Ratio}

Let

$$
\begin{gathered}
\mathrm{E}\left(\mathrm{v}_{\mathrm{t}}\right) \equiv \sum_{\mathrm{i}=0}^{\infty} \mathrm{Z}_{\mathrm{i}} . \\
\frac{z_{i+1}}{z_{i}}=\frac{\exp \left\{-r(i+1+1)+a_{i+1}+\frac{\sigma^{2}}{2\left(1-\rho^{2}\right)} b_{i+1}^{2}\right\}}{\exp \left\{-r(i+1)+a_{i}+\frac{\sigma^{2}}{2\left(1-\rho^{2}\right)} b_{i}^{2}\right\}} \\
=\exp \left\{-\mathrm{r}+\left(\mathrm{a}_{\mathrm{i}+1}-\mathrm{a}_{\mathrm{i}}\right)+\frac{\sigma^{2}}{2\left(1-\rho^{2}\right)}\left(\mathrm{b}_{\mathrm{i}+1}^{2}-\mathrm{b}_{\mathrm{i}}^{2}\right)\right\} .
\end{gathered}
$$

Following from the proof on convergence of the fundamental stock price-dividend ratio $\mathrm{P}_{\mathrm{t}}^{\mathrm{pv}} / \mathrm{D}_{\mathrm{t}}$, it suffices to show that $\lim _{i \rightarrow \infty} \frac{\sigma^{2}}{2\left(1-\rho^{2}\right)}\left(b_{i+1}^{2}-b_{i}^{2}\right)=0$ for a version of Theorem 2.2 in Burnside (1998) to hold. 
We have from Appendix B, $\left(\mathrm{b}_{i+1}^{2}-\mathrm{b}_{\mathrm{i}}^{2}\right)=\frac{\rho^{2}}{(1-\rho)^{2}}\left[\left(1-\rho^{\mathrm{i}+1}\right)^{2}-\left(1-\rho^{\mathrm{i}}\right)^{2}\right]$ which implies $\left(b_{i+1}^{2}-b_{i}^{2}\right)=\frac{\rho^{2}}{(1-\rho)} \rho^{i}\left\{2-(1+\rho) \rho^{i}\right\}$.

Therefore, $\lim _{i \rightarrow \infty}\left(\mathrm{b}_{i+1}^{2}-\mathrm{b}_{\mathrm{i}}^{2}\right)=\lim _{\mathrm{i} \rightarrow \infty} \frac{\rho^{2}}{1-\rho}\left[\rho^{\mathrm{i}}\left\{2-(1+\rho) \rho^{\mathrm{i}}\right\}\right]=0$.

Now, we have $\left|\frac{z_{i+1}}{z_{i}}\right|=\frac{z_{i+1}}{z_{i}}$. Therefore $\lim _{i \rightarrow \infty}\left|\frac{z_{i+1}}{z_{i}}\right|=\exp \left\{-r+\lim _{i \rightarrow \infty}\left(a_{i+1}-a_{i}\right)\right\}$.

From Equation (2.43) we have:

$a_{i+1}-a_{i}=\mu+\frac{\sigma^{2}}{2(1-\rho)^{2}}\left[1-2 \frac{\rho}{1-\rho}\left(1-\rho^{i+1}-1+\rho^{i}\right)+\frac{\rho^{2}}{1-\rho^{2}}\left\{1-\rho^{2(i+1)}-1+\rho^{2 i}\right\}\right]$

$a_{i+1}-a_{i}=\mu+\frac{\sigma^{2}}{2(1-\rho)^{2}}\left[1+\rho^{i+1}\left\{\rho^{i+1}-2\right\}\right]$. Therefore $\lim _{i \rightarrow \infty}\left(a_{i+1}-a_{i}\right)=\mu+$ $\frac{\sigma^{2}}{2(1-\rho)^{2}}$ since $|\rho|<1$. Therefore, $\quad \lim _{i \rightarrow \infty}\left|\frac{z_{i+1}}{z_{i}}\right|=\exp \left\{-r+\mu+\frac{\sigma^{2}}{2(1-\rho)^{2}}\right\}$.

Therefore, from Burnside's (1998) proof of Theorem $1, \sum_{i=0}^{\infty} z_{i}$ converges if $\mathrm{R} \equiv \exp$ $\left\{-\mathrm{r}+\mu+\frac{\sigma^{2}}{2\left(1-\rho^{2}\right)}\right\}<1$

\subsubsection{Appendix D}

\section{Intrinsic Bubbles under AR(1) Process for Dividend Growth Rates}

Equation (2.4) implies $\mathrm{B}_{\mathrm{t}}=\mathrm{e}^{-\mathrm{r}} \mathrm{E}_{\mathrm{t}}\left\{\mathrm{B}_{\mathrm{t}+1}\right\}$. Let $\mathrm{B}\left(\mathrm{D}_{\mathrm{t}}\right)=c \mathrm{D}_{\mathrm{t}}^{\lambda} \exp \left\{h \mathrm{hx}_{\mathrm{t}}\right\}$ where $\lambda$ and $\mathrm{h}$ are constants to be determined. Therefore,

$$
\begin{gathered}
\mathrm{E}_{\mathrm{t}}\left\{\mathrm{B}_{\mathrm{t}+1}\right\}=\mathrm{E}_{\mathrm{t}}\left\{\mathrm{cD}_{\mathrm{t}+1}^{\lambda} \exp \left[\mathrm{hx}_{\mathrm{t}+1}\right]\right\} \\
=\mathrm{E}_{\mathrm{t}}\left\{\mathrm{cD} \mathrm{D}_{\mathrm{t}}^{\lambda} \exp \left[\lambda(1-\rho) \mu+\lambda \mathrm{px}_{\mathrm{t}}+\lambda \xi_{\mathrm{t}+1}\right] \exp \left[\mathrm{h}\left\{(1-\rho) \mu+\rho \mathrm{x}_{\mathrm{t}}+\xi_{\mathrm{t}+1}\right\}\right]\right\}
\end{gathered}
$$




$$
=\mathrm{E}_{\mathrm{t}}\left\{\mathrm{cD}_{\mathrm{t}}^{\lambda} \exp \left[(\lambda+\mathrm{h})(1-\rho) \mu+(\lambda+\mathrm{h}) \rho \mathrm{x}_{\mathrm{t}}+(\lambda+\mathrm{h}) \xi_{\mathrm{t}+1}\right]\right\}
$$

Using the moment generating function of normal random variables, we obtain:

$$
\mathrm{E}_{\mathrm{t}}\left\{\mathrm{B}_{\mathrm{t}+1}\right\}=\mathrm{cD}_{\mathrm{t}}^{\lambda} \exp \left\{(\lambda+\mathrm{h})(1-\rho) \mu+(\lambda+\mathrm{h}) \rho \mathrm{x}_{\mathrm{t}}+(\lambda+\mathrm{h})^{2} \sigma^{2} / 2\right\}
$$

Therefore, the r.h.s. of Equation (2.4) becomes:

$$
\mathrm{cD}_{\mathrm{t}}^{\lambda} \exp \left\{(\lambda+\mathrm{h}) \rho \mathrm{x}_{\mathrm{t}}\right\} \exp \left\{-\mathrm{r}+(\lambda+\mathrm{h})(1-\rho) \mu+(\lambda+\mathrm{h})^{2} \sigma^{2} / 2\right\} .
$$

The 1.h.s. of Equation (2.4) is $\mathrm{B}_{\mathrm{t}}=\mathrm{B}\left(\mathrm{D}_{\mathrm{t}}\right)=\mathrm{cD} \mathrm{D}_{\mathrm{t}}^{\lambda} \exp \left\{h \mathrm{hx}_{\mathrm{t}}\right\}$. Therefore, for Equation (2.4) to hold, we must have:

$$
\mathrm{r}=(\lambda+\mathrm{h})(1-\rho) \mu+(\lambda+\mathrm{h})^{2} \sigma^{2} / 2
$$

and

$$
\mathrm{h}=(\lambda+\mathrm{h}) \rho .
$$


CHAPTER 3

\section{A STATE SPACE FRAMEWORK FOR THE RESIDUAL INCOME VALUATION MODEL OF STOCK PRICES}

\subsection{Introduction}

Understanding equity valuation and expected stock returns have been preeminent topics in asset pricing research for over half a century. The empirical asset pricing literature has largely focused on understanding both cross-sectional and time-series differences in the average rates of stock returns (see Goyal (2012) for a recent survey). One major strand of research within the stock return literature is the use of accountingbased information to explain the cross-sectional behavior of returns. Chen and Zhang (2007) provide theory and evidence to rationalize the link between stock performance and accounting measures of performance. Within a wide array of accounting information, canonical work, particularly Fama and French (1993, 1995, 1996, 1998), suggests that book-to-market, market capitalization, and earnings yields can explain much of the stock return variation. While expected-return models are crucial to gain further understanding of stock market dynamics, equally important are stock valuation models.

Bakshi and Ju (2002) distinctly note that stock valuation models that support the intrinsic link between returns, book values, and earnings "can provide the much-needed impetus for reconciling the behavior of expected returns and market valuations." One such stock valuation model is the discounted residual income model (RIM or RI), formalized by Ohlson $(1991,1995)$. The RIM is built upon the traditional dividend discount model. It focuses on firms' earning power and wealth creation, as captured by accounting data, rather than the expected dividend sequence. It assumes the clean surplus relation (CSR), which posits that the change in book value of equity is equal to retained 
earnings. 'Residual income' or 'abnormal earnings' ${ }^{1}$, is defined as the difference between accounting earnings and the previous-period book value multiplied by the cost of equity.

The residual income model implies that the current stock price equals the current book value of equity plus the expected present discounted value of all future residual income. One of the main ingredients of Ohlson (1995) is an explicit formulation of residual income information dynamics. The chief feature of information dynamics is that expected next period $t+1$ residual income contains information known at time $t$ that is not present in current residual income. Dechow et al. (1999) (henceforth DHS) provide an empirical evaluation of the Ohlson (1995) model, explicitly including residual income information dynamics for the first time in their study. They use Wall Street consensus analysts' earnings forecasts as a measure to estimate the so-called 'other information' about next period $t+1$ earnings. They argue that Wall Street equity analysts hold better predictive information on firms' earnings relative to retail investors.

Naturally, investors find information about a firm's future earnings prospects highly important to their stock selection process. Therefore, they value consensus analysts' earnings forecasts as a conveyor of or signal for information about a stock. Despite their importance to investors, such use of earnings forecast data have several drawbacks, including bias and subjectivity from competing stock analysts, limited data availability, ample heterogeneity in analysts' reputation, and credibility of their forecasts within the aggregate market. Furthermore, frequent conflicting interests of the analystmanagement relationship has been problematic for decades, hampering unbiased stock recommendations. Recent regulatory reform, namely the NASD Rule 2711 mandates analyst independence in order to enhance the objectivity of analyst recommendations. However, such regulations have induced unintended consequences, particularly the reduction of market-wide research coverage. They have also widened information asym-

\footnotetext{
${ }^{1}$ We use residual income and abnormal earnings interchangeably throughout the paper.
} 
metry between retail investors and wealthier, highly-sophisticated institutional investors, who have access to costly information sources (see Fisch (2007) for detailed discussion). In light of this, we ask what are the alternative measurements or estimation methods for modelling 'other information' in the residual income valuation model?

The purpose of this paper is to apply the residual income valuation model for equity prices developed in Ohlson (1995) and assess the empirical implications of the model by estimating the 'other information' variable using a state space framework. We estimate the valuation model with the embedded state space framework using the Kalman filter across a sample of stocks, namely, in the Dow Jones 30 (henceforth DJIA) and S\&P 500 indices. We compare model performance to the benchmark two-step regression approach used in DHS. Our empirical results provide comparative performance of the two approaches in forecasting next period $t+1$ abnormal earnings, current period $t$ other information, and current period $t$ stock prices, both in- and out-of-sample.

We organize the paper as follows. In Section 3.2, we provide a general framework for both the dividend discount model and the residual income model, and discuss extensions of Ohlson's RI model. We also present the benchmark DHS estimation method along with a discussion of its drawbacks, followed by an introduction to our state space (henceforth SS) estimation method. In Section 3.3, we discuss the data, and report empirical results along with inferences drawn from our investigations. We summarize our main findings and briefly consider plausible extensions of the paper in the concluding section. 


\subsection{Valuation Models}

\subsubsection{The Dividend Discount Model}

In finance, the conventional equity valuation model is the dividend discount model, which specifies that stock price is equal to the present value of expected future dividends stream:

$$
P_{t}=\sum_{\tau=1}^{\infty} \frac{\mathrm{E}_{t}\left[d_{t+\tau}\right]}{(1+r)^{\tau}},
$$

where $P_{t}$ is the price of the firm's equity at time $t, d_{t}$ is net dividends paid at time $t$, $r$ is the constant discount rate, and $E_{t}[\cdot]$ is the expected value operator conditional on information set available at date $t$.

The subject of market efficiency that promotes the idea that stock prices reflect rational investor responses to all new information about fundamentals has received critical attention over virtually four decades. Such market efficiency tests were pioneered by the stock price volatility tests of LeRoy and Porter (1981) and Shiller (1981). Based on Eq. (3.1), they found that stock market volatility was far greater than could be justified by subsequent changes in dividends. A number of studies provide further substantive evidence that stock price fluctuations are simply too excessive to result solely from fluctuations in the expected present discounted value of dividends. For example, West (1988) developed a variance-bound test that avoids small sample bias and is valid even when the cash (or ordinary) dividends stream does not mean-revert. Campbell and Shiller (1987) derived testable implications of the present value model, taking into account the nonstationarity and cointegration of stock prices and ordinary dividends. Both West and Campbell and Shiller found strong evidence against the simple present value relation in which ordinary dividends are used as the discounted cash flow. 
However, Ackert and Smith (1993) show that inferences from volatility tests depend on how econometricians measure dividend distributions to shareholders. As Miller and Modigliani (1961) have established, dividends generally represent all cash distributions to shareholders, including proceeds from share repurchases and mergers and acquisitions. Empirically, this would make the dividend distribution a much broader set. As a result, Ackert and Smith (1993), using broad dividends inclusive of other cash distributions, rather than ordinary, cash dividends solely as done in previous studies, show an improvement in the performance of the present value model. In support of the use of broad dividends, it is worth noting that Fama and French (2001) provide evidence that the number of firms paying cash dividends has drastically declined since the late 1970s. In fact, Grullon and Michaely (2002) show that share repurchase activity has grown considerably since the mid-1980s, and has become a substitutable form of cash payout channel for publicly-traded US firms.

Such dynamic shifts in dividend measurement illuminate the limitations of using the dividend discount model to value stocks. This motivates the necessity of a discounted cash flow present value framework that can broadly capture total cash distributions paid to shareholders, not just conventional cash dividends.

\subsubsection{Residual Income Valuation Model}

In accounting literature, an alternative valuation model, the residual income valuation model (RIM or RI), has become widely popular among theoretical and empirical researchers, primarily due to its formalization by Ohlson $(1991,1995)$. The RIM assumes an integral accounting identity, namely, the clean surplus relation (CSR), which stipulates that the change in book value of equity is equal to the difference between accounting earnings and dividends. The residual income (or abnormal earnings) is defined as the dif- 
ference between current accounting earnings and the product of previous-period book value and the cost of capital. RIM implies that the current stock price equals the current book value of equity plus the expected present value of all future residual income. A critical feature of the RIM is that dividends, via the CSR, are defined broadly as the difference between earnings and the change in book value of equity. As such, dividends in the RIM include not only traditional ordinary, cash dividends, but also other forms of cash distributions to shareholders (e.g., share repurchases, mergers, acquisitions, etc.).

\section{Model Overview}

The CSR assumption is important for the RIM in order to express goodwill (i.e. the difference between firm market value and its book value) as a function of expected next period $t+1$ residual income. The CSR stipulates that all gains and losses of a firm's book value be incorporated in retained earnings; in other words, a one-period change in book value equals earnings minus dividends. The CSR accounting identity is expressed as:

$$
b_{t}=b_{t-1}+x_{t}-d_{t}
$$

where $b_{t}$ is the book value of equity at time $t, x_{t}$ is earnings for the period from $t-1$ to $t$, and $d_{t}$ is net dividends paid out at time $t$.

Combining Eq. (3.2) with the present value stock price in Eq. ((3.1) by substituting out for $d_{t}$ yields:

$$
P_{t}=\sum_{\tau=1}^{\infty} \frac{\mathrm{E}_{t}\left[b_{t+\tau-1}+x_{t+\tau}-b_{t+\tau}\right]}{(1+r)^{\tau}}
$$

On forward iteration, Eq. (3.3) can be reduced to:

$$
P_{t}=b_{t}+\sum_{\tau=1}^{\infty} \frac{\mathrm{E}_{t}\left[x_{t+\tau}-r \cdot b_{t+\tau-1}\right]}{(1+r)^{\tau}}-\underbrace{\frac{E_{t}\left[b_{t+\infty}\right]}{(1+r)^{\infty}}}_{0}
$$


Imposing the transversality condition on the last term in Eq. (3.4) guarantees that the book value stream is not an explosive one. For this condition to be satisfied, given the clean surplus accounting relation, dividends will have to be paid at some future date. As such, imposing the transversality condition allows one to define residual income $x_{t}^{a}$ as earnings minus a charge for the use of capital, as measured by start-of-period book value of equity multiplied by the cost of capital (assumed to be the constant discount rate, $r)$ :

$$
x_{t}^{a}=x_{t}-r \cdot b_{t-1}
$$

As such, we can specify the residual income model as:

$$
P_{t}=b_{t}+\sum_{\tau=1}^{\infty} \frac{\mathrm{E}_{t}\left[x_{t+\tau}^{a}\right]}{(1+r)^{\tau}}
$$

which states that the current stock price equals the sum of current book value of equity and the present value of all expected future abnormal earnings. The latter term reflects a firm's future profitability. One should note that Eq. (3.6) is simply a restatement of the dividend discount model. Specifically, it does not depend on the properties of the accounting variables, other than by way of the CSR and the transversality condition imposed in Eq. (3.4).

Using accounting values as a fundamental information framework for investors, Ohlson (1995) formulates dynamic linear recursive equations in order to relate current accounting numbers with future expected accounting information. Specifically, he assumes that abnormal earnings satisfy the following modified autoregressive process: 


$$
\begin{gathered}
x_{t}^{a}=\omega x_{t-1}^{a}+v_{t-1}+\varepsilon_{t}, \quad \varepsilon_{t} \sim i i d N\left(0, \sigma_{\varepsilon}^{2}\right) \\
v_{t}=\gamma v_{t-1}+\eta_{t}, \quad \eta_{t} \sim \operatorname{iid} N\left(0, \sigma_{\eta}^{2}\right)
\end{gathered}
$$

where $\nu_{t}$ is 'other information' about next period $t+1$ abnormal earnings $x_{t+1}^{a}$ not conveyed in current abnormal earnings $x_{t}^{a}, \varepsilon_{t}$ and $\eta_{t}$ are unpredictable, mean zero disturbance terms, and $\omega$ and $\gamma$ are fixed persistence parameters, assumed non-negative and less than one.

Combining the residual income valuation model in Eq. (3.6) with the information dynamics in Eqs. (3.7a) and (3.7b) yields the following implied valuation function:

$$
P_{t}=b_{t}+\alpha_{1} x_{t}^{a}+\alpha_{2} v_{t}
$$

where $\alpha_{1}=\omega /(1+r-\omega)$ and $\alpha_{2}=(1+r) /[(1+r-\omega)(1+r-\gamma)]$. This valuation function is consistent with the ModiglianiâĂŞMiller dividend irrelevance theorem. Ohlson's (1995) formulation of information dynamics in Eq. (3.7) implies that unconditional expected goodwill is zero. That is, even if there is persistence in goodwill for long periods into the future, its average value approximates to zero. Therefore, book value of equity is an unbiased estimator of market value of equity. Eq. (3.8) implies that the market value for a firm reflects the book value adjusted for current abnormal earnings and 'other information' about future profitability.

\section{Extensions of Ohlson's RI Model}

While several empirical applications of the residual income valuation model have surfaced in the literature, several of them unfortunately ignore the information dynamics formulated in Ohlson (1995). Consequently, these valuation models effectively become 
applications of the dividend discount model, in which current earnings are assumed to be the sole measure of 'wealth creation'. As such, they overlook book value or abnormal earnings (see, for example Whitbeck and Kisor, 1963; Malkiel and Cragg, 1970; Easton and Harris, 1991; Kothari and Zimmerman, 1995).

Since Dechow et al. (1999) (DHS), however, recent works have incorporated residual income information dynamics in addition to value-relevant information, and empirically do a better job than competing variants of the dividend discount model. For instance, Tsay et al. $(2008,2009)$ incorporate non-earnings information variables (e.g. bankruptcy costs, earnings components, and growth opportunities) in addition to consensus analysts' earnings forecasts. They find better predictive ability and estimation efficiency in explaining contemporaneous stock prices by incorporating such additional information. Lyle et al. (2013) incorporate dynamic expectations about the level of systematic risk in the economy. Balachandran and Mohanram (2012) use residual income (RI) to decompose earnings growth into several growth factors (e.g. RI growth and invested capital growth). They then use this decomposition to explain stock returns. Additional work uses various econometric techniques to test the validity and empirical performance of the RI model (see Jiang and Lee (2005)), and adjusts for sources of bias to forecast RIbased stock prices (see Higgins (2011) and Kuo (2016)).

Several theoretical extensions, including but not limited to Feltham and Ohlson (1999), Ang and Liu (2001), and Gode and Ohlson (2004), lay a solid foundation allowing for stochastic interest rates and risk, in addition to information dynamics, in the residual income model. Recent work by Bergeron et al. (2018) incorporates the now popular concept of long-run risk, pioneered by Bansal and Yaron (2004), into the residual income valuation framework.

In this paper, we reassess the residual income valuation model analyzed in DHS, by utilizing a state space approach to extract the latent 'other information' variable in 
Ohlson's model, instead of proxying it with consensus analyst forecasts of next period $t+1$ earnings, as originally done in DHS.

\subsubsection{DHS Estimation Method}

The original, empirical implementation of information dynamics and implied valuation function in Eqs. (3.7a and 3.7b) and (3.8), respectively, in DHS involves three variables $\left(b_{t}, x_{t}\right.$, and $\left.\nu_{t}\right)$ and three parameters $(\omega, \gamma$ and $r)$. The first two variables, $b_{t}$ and $x_{t}$, are readily observed and easily measured. However, the last variable $\nu_{t}$ and the three parameters are unobserved. These are to be estimated. The underlying idea of Ohlson (1995) is that a firm's stock price reflects information about its next period $t+1$ profitability that is not readily incorporated in current profitability. As such, Ohlson defines the 'other information' variable, $\nu_{t}$, as the difference between the conditional expectation of abnormal earnings for next period $t+1$ based on all available information at time $t$ and that based on current period $t$ abnormal earnings alone:

$$
v_{t}=\mathrm{E}_{t}\left[x_{t+1}^{\mathrm{a}}\right]-\omega x_{t}^{\mathrm{a}}
$$

The conditional expectation of next period $t+1$ abnormal earnings, denoted as $f_{t}^{a}$ is equal to the conditional expectation of next period $t+1$ earnings at time $t$ less the product of book value at time $t$ and the discount rate. DHS measure the conditional expectation of next period $t+1$ earnings at time $t$ using the consensus analyst forecast of next period $t+1$ earnings, denoted as $f_{t}$, in which case

$$
\mathrm{E}_{t}\left[x_{t+1}^{\mathrm{a}}\right]=f_{t}^{\mathrm{a}}=f_{t}-r \cdot b_{t}
$$


Therefore, from Eqs. (3.9) and (3.10), 'other information', $\nu_{t}$ can be retrieved as

$$
v_{t}=f_{t}^{a}-\omega x_{t}^{\mathrm{a}} .
$$

The values for the three parameters, namely $\omega, \gamma$, and $r$ are pinned down as follows. The authors use the average historical return on equities to measure the discount rate, $r$. They estimate the persistence parameters, $\omega$ and $\gamma$, which they postulate as common across firms, by using unconditional pooled regressions.

$$
\begin{aligned}
& x_{i, t}^{\mathrm{a}}=\omega_{0}+\omega_{1} x_{i, t-1}^{\mathrm{a}}+\varepsilon_{1 i, t}, \quad \varepsilon_{1 i, t} \sim \text { iid } N\left(0, \sigma_{1}^{2}\right) \\
& v_{i, t}=\gamma_{0}+\gamma_{1} v_{i, t-1}+\varepsilon_{2 i, t}, \quad \varepsilon_{2 i, t} \sim \operatorname{iid} N\left(0, \sigma_{2}^{2}\right) .
\end{aligned}
$$

$x_{i, t}^{a}$ is current period $t$ abnormal earnings for firm $i . v_{i, t}$ is 'other information' about next period $t+1$ abnormal earnings known at time $t$ for firm $i . \omega_{0}$ and $\gamma_{0}$ are the intercepts. $\omega_{1}$ and $\gamma_{1}$ are the persistence parameters. $\varepsilon_{i 1, t}$ and $\varepsilon_{i 2, t}$ are error terms for firm $i$ with a zero mean and variances $\sigma_{1}^{2}$ and $\sigma_{2}^{2}$, respectively.

The two-step regression approach of DHS proceeds as follows. First, Eq. (3.12a) is estimated. The estimate of omega $_{1}$ is used as an estimate of $\omega$ in Eq. (3.7a). Using the estimate of $\omega_{1}$, from Eq. (3.12a) for $\omega$ in Eq. (3.11), a time series for $\nu_{t}$ is constructed. Using this series for $\nu_{t},(3.12 \mathrm{~b})$ is then estimated in the second step. The estimate of $\gamma_{1}$ is then used as an estimate of $\gamma$ in Eq. (3.7b).

In essence, in the DHS methodology, separate two-step estimation of Eqs. (3.12a) and (3.12b) serves as the foundation of their empirical analysis. It enables calculation of the implied valuation function in Eq. (3.8). This approach serves as a benchmark for our alternative SS estimation approach. 
From the standpoint of Ohlson's theory, it is important to mention that this empirical strategy of DHS in question is a flawed one. As denoted in Eqs. (3.7a) and (3.7b) of section 3.2.2, a key assumption regarding information dynamics in the RIM framework stipulates that next period $t+1$ abnormal earnings are a linear function of current period $t$ abnormal earnings and a latent variable. This 'other information' variable about next period $t+1$ abnormal earnings is posited to evolve as a first-order autoregressive process. Therefore, estimates of the persistence parameter $\omega_{1}$ in Eq. (3.12a), obtained from an unconditional pooled regression for abnormal earnings, is contaminated. Furthermore, the second step estimation of $\gamma_{1}$ from Eq. (3.12b) is also problematic from an econometric viewpoint.

Use of the two-step regression in DHS, as exemplified by Eqs. (3.12a) and (3.12b), leads to the well-known drawback, namely, classical measurement error on generated variables, and the efficiency and consistency loss that ensues (see, for example Pagan (1984); Oxley and McAleer (1993); and Hausman (2001) for related discussions). As a result, empirical analyses of the residual income valuation models that employ consensus analysts' earnings forecasts to extract the 'other information' variable $\nu_{t}$ are susceptible to this drawback in methodology.

Our paper seeks to address two drawbacks in the RIM literature. One is to avoid using analysts' earnings forecasts data in favor of an objective model-based approach to estimate 'other information' $\nu_{t}$. Another is to avoid drawbacks of the two-step estimation employed by DHS. We utilize a state space approach to extract the unobserved other information $\nu_{t}$ in the next section. The application of this technique serves as the paper's main contribution to the residual income valuation literature. 


\subsubsection{SS Estimation Method}

The dynamic evolution of abnormal earnings is specified in Equations (3.7a) and (3.7b). Eqs. (7a) and (7b) can be represented in general state space form in which the measurement (observation) equation relating $y_{t}$ to the latent unobserved variable $\alpha_{t}$ is formulated as:

$$
\underset{n \times 1}{y_{t}}=z_{t} \alpha_{t}+\underset{n \times 1}{\varepsilon_{t},} \quad \varepsilon_{t} \sim i i d N\left(\underset{n x 1}{\overline{0}}, H_{t}\right)
$$

and the transition (state) equation describing the evolution of the latent variable can be formulated as:

$$
\underset{(n+1) \times 1}{\alpha_{t}}=T_{t} \underset{(n+1) \times 1}{\alpha_{t-1}}+R_{t} \underset{(n \times 1)}{\eta_{t}}, \quad \eta_{t} \sim i i d N\left(\underset{n x 1}{\overline{0}}, Q_{t}\right)
$$

Here, $y_{t}$ is the column vector of observed abnormal earnings at time $t . z_{t}=\left(\begin{array}{ll}I_{n} & y_{t-1}\end{array}\right)$ is a $n \times(n+1)$ matrix containing a $n$-dimensional identity matrix and column vector of lagged observed abnormal earnings at time t. $\alpha_{t}=\left(\begin{array}{c}v_{t} \\ \omega\end{array}\right)$ is the $(n+1) \times 1$ state vector of $\nu_{t}$, the 'other information variable' in the RIM, and $\omega$, the unknown persistence parameter of abnormal earnings appearing in Eq. (3.7a). $H_{t}=\sigma_{\varepsilon}^{2} I_{n}$ is the covariance matrix for the measurement noise $\varepsilon_{t} . T_{t}=\left(\begin{array}{cc}\gamma I_{n} & 0 \\ 0 & 1\end{array}\right)$ is a $(n+1) \times(n+1)$ matrix of parameters governing the state transition. $R_{t}=\left(\begin{array}{c}I_{n} \\ \overline{0}^{\prime}\end{array}\right)$ is a $(n+1) \times n$ matrix of parameters and $Q_{t}=\sigma_{\eta}^{2} I_{n}$ is the covariance matrix for the signal shock $\eta_{t} . H_{t}$ and $Q_{t}$ are serially and mutually independent. 
A scalar state space representation for Eqs. (3.7a) and (3.7b), for a single firm $n=1$, along with the Kalman filtering procedure that we have implemented for econometric analysis can be found in the Appendix. ${ }^{2}$

Given the linear Gaussian state space specification above, we estimate the unknown parameters contained in the vector, $\phi=\left\{\omega, \gamma, \sigma_{\varepsilon}^{2}, \sigma_{\eta}^{2}\right\}$, as well as the latent state vector $\alpha_{t}$, using the Kalman filter.

Initialization. We initialize the Kalman filter with the initial state, $a_{t \mid t}$ and $p_{t \mid t}$ at $t=0$. Let $a_{t \mid t}=E\left[\alpha_{t} \mid Y_{t}\right]$, where $Y_{t}=\left\{y_{t}, y_{t-1}, y_{t-2}, \ldots y_{1}\right\}$, be the filter mean of unobserved state $\alpha_{t}$, given observations up to and including $y_{t}$, and $p_{t \mid t}$ be the $n \times n$ covariance $\operatorname{matrix} p_{t \mid t}=\operatorname{var}\left(\alpha_{t} \mid Y_{t}\right)$.

Prediction. Given $a_{t \mid t}$ and $p_{t \mid t}$, the one-step ahead predictor ${ }^{3} a_{t+1 \mid t}$ is provided by:

$$
\begin{aligned}
a_{t+1 \mid t}= & E\left(\alpha_{t+1} \mid Y_{t}\right) \\
& =E\left(T_{t+1} \alpha_{t}+R_{t+1} \eta_{t+1} \mid Y_{t}\right) \\
& =T_{t+1} \cdot a_{t \mid t} \\
p_{t+1 \mid t}= & \operatorname{var}\left(\alpha_{t+1} \mid Y_{t}\right) \\
= & \operatorname{var}\left(T_{t+1} \alpha_{t}+R_{t+1} n_{t+1} \mid Y_{t}\right) \\
= & T_{t+1} p_{t \mid t} T_{t+1}^{\prime}+R_{t+1} Q_{t+1} R_{t+1}^{\prime}
\end{aligned}
$$

${ }^{2}$ Note that the sum of the $\log \mathrm{L}$ for either state space form match as the former estimates the $\log \mathrm{L}$ for all firms in the sample concurrently, while the latter does so individually for each firm $\mathrm{j}$ at a time.

${ }^{3}$ Note we lose a degree of freedom in the time-series for each stock in the respective sample when calculating a one step-ahead prediction. 
Filtering. Once a new observation $y_{t+1}$ arrives, the one-step ahead predictions are updated using the filtering equations:

$$
\begin{aligned}
& a_{t+1 \mid t+1}=a_{t+1}+\left(p_{t+1} z_{t+1}^{\prime} F_{t+1}^{-1}\right) \varphi_{t+1} \\
& p_{t+1 \mid t+1}=p_{t+1}-p_{t+1} z_{t+1} F_{t+1}^{-1} z_{t+1}^{\prime} p_{t+1}
\end{aligned}
$$

where $\varphi_{t+1}=y_{t+1}-z_{t+1} a_{t+1}=y_{t+1}-E\left(y_{t+1} \mid Y_{t}\right)$ is the one-step ahead prediction error and $F_{t+1}=\operatorname{var}\left(\varphi_{t+1}\right)=z_{t+1} p_{t+1} z_{t+1}^{\prime}+H_{t+1}$ is its conditional variance. Eqs. (3.14) and (3.15) together represent the Kalman filtering recursive procedure, whose application generates the filter and predictive means and variances of the state variables $\alpha_{t}$, conditional on $\phi=\left\{\omega, \gamma, \sigma_{\varepsilon}^{2}, \sigma_{\eta}^{2}\right\}$.

Log-likelihood construction. As a by-product of the above recursive predictive and filtering equations, we can recursively compute $f\left(y_{t} \mid Y_{t-1}, \phi\right)$ for $t=1,2, \ldots, N$ from the normal probability density function. We can use these densities to construct the log-likelihood as follows:

$$
\log L=-\frac{n N}{2} \log (2 \pi)-\frac{1}{2} \sum_{t=1}^{N} \log \left|F_{t}\right|-\frac{1}{2} \sum_{i=1}^{N} \varphi_{t}^{\prime} F_{t}^{-1} \varphi_{t}
$$

where $\left|F_{t}\right|$ is the determinant of $F_{t}$. We use numerical methods to minimize the negative of the log-likelihood function in Eq. (3.16), with respect to the unknown vector of parameters $\phi$. 


\subsection{Empirical Assessment of the Model}

\subsubsection{Data Description, Aggregation, Transformation, and Clean- ing Details}

\section{Data Description}

To investigate the empirical performance of our proposed SS estimation method, we use a sample of constituents of major US stock indices, namely the DJIA and the S\&P 500 , from a couple of sets of accounting and stock market databases. We subsequently clean, aggregate, and transform the raw sample data.

For historical stock price and accounting data, we use annual 1950-2017 CRSP and COMPUSTAT Merged data files. To identify the constituents of the DJIA and S\&P 500 as of 2017 fiscal year-end, we use COMPUSTAT Daily Updates - Index Constituents data files. Additionally, for analyst forecast data, we retrieve annual 1976-2017 I/B/E/S data files. In order to implement the benchmark DHS estimation method, the I/B/E/S files are a mandatory requirement since they contain analyst forecast data. An evident issue here is the unavailability of analyst forecast data before 1976. However, for state space estimation, we only need the 1950-2017 CRSP/COMPUSTAT data. This fact further motivates the usefulness of the state space approach, as it eliminates the need for additional data sets that may be subject to limited availability.

Since the set of available analyst forecasts within the sample period is vast, we use only the latest analyst forecast recorded for each forecast period.

We conduct the empirical evaluation using per-share accounting data, including book value per share (bvps), earnings per share (eps), and closing stock prices per share. Each accounting variable is adjusted for periodic stock events, including stock splits, stock dividends, mergers and acquisitions, etc. We additionally measure each variable 
based on fiscal year-end; otherwise, if unavailable for a firm-year observation, we measure it using calendar year-end. All analyses use basic earnings measured before extraordinary items, and a discount rate of $12 \%$ as adopted by DHS, which they approximate to be the long-run average realized return on US equities.

We construct an abnormal earnings series by using data for earnings and book value, and the assumed cost of equity at $12 \%$ in Eq. (3.5).

We note two things for the empirical strategy in DHS: all relevant variables are scaled by market value of equity to control for heteroscedasticity and the $1 \%$ most extreme observations are winsorised in order to mitigate their undue effect on regression estimates. However, for our purposes, we do not follow their scaling and winsorization procedure. The use of winsorization and scaling can be riddled with several pitfalls, which may jeopardize the validity of results. Such pitfalls include the hazard of a skewed distribution of the information set in sample, and the potential tradeoff between forecast accuracy and economic value of such regression-based forecasts (see Brownen-Trinh (2019) for in-depth discussion). Robust estimators such as least trimmed squares estimate, TheilSen estimator (see Theil (1950 and Sen (1968)), and robust scale minimization (see Peña and Yohai (1999) for procedure) can overcome the challenges of scaling and winsorization, and tend to perform well in spite of any outliers that may be present. Employing such robust estimators in the context of the SS model here goes beyond the scope of this study.

\footnotetext{
${ }^{4}$ Note we lose a degree of freedom in the time-series for each stock in the respective sample when aggregating abnormal earnings. Constructing abnormal earnings at time $t$ requires book value at time $t-1$. Therefore, given that the sample period of 1950-2017 totals 68 time periods, we can have no more than 67 time periods in the abnormal earnings data series for each stock.
} 


\section{Data Aggregation and Transformation Details}

We begin aggregating the original data by extracting the CRSP/Compustat Merged

- Fundamentals Annual data file for active companies only during the January 1950 to December 2017 fiscal year period. We select the following array of relevant variables $^{5}$ to be generated within the extracted data set: GVKEY, ${ }^{6}$ Historical CRSP PERMCO Link to COMPUSTAT Record (LPERMCO), Historical CRSP PERMNO Link to COMPUSTAT Record (LPERMNO), Company Name (CONM), Ticker Symbol (TIC), CUSIP, North American Industry Classification Code (NAICS), Standard Industry Classification Code (SIC), Adjustment Factor (Company) - Cumulative by Ex-Date (AJEX), Fiscal Year (FYEAR), Book Value Per Share (BKVLPS), Earnings Per Share (Basic) Excluding Extraordinary Items (EPSPX), Price Close - Annual - Calendar (PRCC_C), and Price Close - Annual - Fiscal (PRCC_F). Using the company identifier (GVKEY) to relate the two data files, we identify the constituents of the DJIA and S\&P 500 indices in the CRSP/COMPUSTAT Merged data file with the COMPUSTAT Daily Updates - Index Constituents data file, where there are 30 constituents within the Dow Jones Industrial Average and 501 constituents within the S\&P $500 .^{7}$

Within the CRSP/COMPUSTAT Merged data file, we create an adjusted version of each per share data variable (i.e. price and earnings per share). The adjusted variables are data transformations that account for all stock splits and stock dividends that occurred subsequent to the end of a given period. To do so, we adjust the per share data and share data by dividing and multiplying them by the adjustment factor (AJEX), respectively for each time period.

\footnotetext{
${ }^{5}$ See Appendix for detailed list of variable names and descriptions relevant to the analysis.

${ }^{6}$ Automatically included in data extract.

${ }^{7}$ See Appendix for full list of constituents for each index.
} 
Note that we have two share price variables, PRCC_C and PRCC_F. We primarily use the closing price based on fiscal year-end (PRCC_F); however, if data are missing at a specific time period, then we substitute it with the closing price at calendar year-end (PRCC_C).

To follow the DHS estimation method, we extract the $t+1$ analyst earnings forecast data in the I/B/E/S - Summary History - Summary Statistics data file during the January 1976 to December 2017 forecast period. We select the following array of relevant variables to be generated within the extracted data set for analyst earnings forecast: CUSIP (8-digit), Forecast Period End Date, IBES Statistical Period, and Mean Estimate. ${ }^{8}$ Again, since the set of available analyst forecast universe is rather large, we use only the most recent, mean analyst forecast estimate recorded for each forecast period in the data extract. Using a combination of company identifiers across the databases (GVKEY, LPERMCO, and NCUSIP), we merge the $t+1$ analyst earnings forecast in I/B/E/S with our CRSP/COMPUSTAT Merged data file. ${ }^{9}$ This produces a total of 1,515 firm-year observations of the DJIA sample and 18,247 firm-year observations of the S\&P 500 sample.

\section{Data Cleaning Details}

To clean the merged data, we remove all firm data if it contains missing observations, gaps in the time-series, negative prices or book values, or less than three years of data. As such, we end up with 19 DJIA stocks totalling 853 firm-year observations and 286 S\&P 500 stocks totalling 9,093 firm-year observations. ${ }^{10}$ Note that all stocks under the

\footnotetext{
${ }^{8}$ See Appendix for detailed list of variable names and descriptions relevant to the analysis.

${ }^{9}$ To merge the analyst forecast data from I/B/E/S with CRSP/COMPUSTAT Merged data files in order to complete our compilation successfully is not straightforward and as such requires several steps. This matter is a commonly known issue and a thorough solution can be found here: https://wrds-www.wharton.upenn.edu/pages/support/research-wrds/ linking-ibes-and-crsp-data/\#using-link-tables-to-merge-ibes-and-crsp-data.

${ }^{10}$ See Appendix for full list of stocks included in sample set.
} 
Dow Jones Industrial Average are also under the S\&P 500. Therefore the 19 stocks used in the DJIA sample are used in the S\&P 500 sample too. ${ }^{11}$

We conduct our analysis by splitting the aggregate sample into two sub-samples: firms only in the financial, insurance, and public utility (henceforth FIPU) sectors and firms outside those sectors (henceforth non-FIPU). We do this because the quantitative characteristics of financial data for financial institutions, insurance companies, and public utilities tend to be substantially different from those of other types of firms. An aggregate sample inclusive of FIPU firms may skew the distribution of each financial variable employed in our empirical evaluation. As such, we have 14 (5) non-FIPU (FIPU) stocks in the DJIA sub-sample and 216 (70) non-FIPU (FIPU) stocks in the S\&P 500 sub-sample.

\section{Summary Statistics}

Tables 3.1 and 3.2 provide summary statistics on key financial variables for non-FIPU and FIPU stocks employed in the analysis, respectively. The mean stock price for nonFIPU (FIPU) firms traded under each index is over three times (nearly two times) the mean value of book value per share. This potentially indicates that investors see market price as a forward-looking metric, reflecting information on expected next period $t+1$ earnings beyond the cost of capital and book value of equity (see Penman (1996) for related discussion). This motivates the merit and practicality of the residual income valuation model, which asserts that stock price is the sum of book value and the discounted present value of expected abnormal earnings.

The five measures of variability for virtually all of the financial variables collectively indicate larger dispersion for FIPU stocks relative to non-FIPU stocks. This is particularly true for FIPU stocks under the S\&P 500, driven predominantly by significant finan-

\footnotetext{
${ }^{11}$ We use these 19 stocks in addition to 5 others arbitrarily chosen for graphical illustrations in the paper.
} 
cial losses reported by insurance giant AIG in 2008. This larger dispersion, coupled with larger third and fourth central moments for FIPU firms, further justifies the need to split the sample into two sub-samples, as done here.

Abnormal earnings is less than earnings per share on average for firms traded under each index by construction, underlying the critical assumption of the residual income valuation model that earnings should account for the true cost of capital. That is, it should not only capture the cost of debt (i.e. interest expenses readily included in net income calculation), but also the cost of equity (i.e. cash distributions to shareholders not readily included in net income calculation).

The first two central moments of analysts' earnings forecast for non-FIPU stocks traded under both the DJIA and S\&P 500 are comparable to those of actual earnings per share. This sheds some light on the forecasting abilities of Wall Street analysts for firms outside of the financial, insurance, and utilities sectors. We also find similarities in the first two central moments for FIPU firms traded under the DJIA, but not so much for those traded under the S\&P 500. This, of course, is not surprising considering that most financial and insurance firms, including AIG, reported record losses during the 20072009 financial crisis.

The average $\mathrm{P} / \mathrm{E}$ ratio for non-FIPU (FIPU) stocks traded under the $\mathrm{S} \& \mathrm{P} 500$ is roughly $\$ 0.08$ (\$1) above that of blue chip stocks. ${ }^{12}$ This may signify confidence in future performance investors have in stocks traded in the broader index, and thus the premium they are willing to pay in shares per dollar generated in earnings. The mean RI/E ratio for non-FIPU stocks shows that a dollar in earnings generates approximately 16-19 cents in abnormal profits. Yet, the mean RI/E ratio for FIPU stocks shows a 4-17 cent residual income loss per dollar generated in earnings. It is worth remembering that residual income

\footnotetext{
${ }^{12}$ We use 'blue chip stocks' interchangeably with 'stocks traded under the DJIA index' or any similar variation.
} 
is excess earnings, after accounting for the equity capital cost. Therefore, this result is suggestive of the average historical performances for non-FIPU and FIPU firms. The historical performance of the former may signal to investors interested in diversification its relative lucrativeness when compared to the latter.

A stylized fact documented in the empirical finance literature is the non-Gaussian feature of financial data. All variables in each table exhibit strong leptokurtosis, highlevels of skewness ranging from -13.736 to 21.048 (-44.428 to 21.507) in Table 3.1 (Table 3.2), with normality unequivocally rejected at a $1 \%$ significance level. Figures 3.1 and 3.2 and 3.3 and 3.4 plot the raw data of the financial variables for the 19 DJIA and 286 S\&P 500 stocks, respectively.

\subsubsection{Model Estimates}

We begin our empirical analysis by analyzing how well abnormal earnings are described by the information dynamics characterized by Eqs. (3.7a) and (3.7b). Joint estimation of Eqs. (3.7) is accomplished by the SS method proposed in this study. Benchmark two-step estimation, as in DHS, is also undertaken for comparison. We report estimates of the persistence parameters $\omega$ and $\gamma$, as well as those of the variances of shocks to the abnormal earnings process and 'other information' dynamics $\sigma_{\epsilon}^{2}$ and $\sigma_{\eta}^{2}$, respectively.

Table 3.3 presents maximum likelihood (ML) parameter estimates for the SS method described in section 3.2.4. Pooled time-series and cross-sectional regression estimates for the DHS method described in section 3.2.3 are also reported. Panels A and B provide parameter estimates for non-FIPU and FIPU firms, respectively. 


\section{Estimates of Abnormal Earnings for Non-FIPU Stocks}

For the SS method in Panel A, the persistence parameter for $x_{t}^{a}$, $\omega$ is 0.0139 and 0.003 with a standard error of 0.1177 and 0.0019 for DJIA and S\&P 500, respectively. The persistence parameter for $\nu_{t}, \gamma$ is 0.8967 and 0.4727 with a standard error of 0.0242 and 0.0102 for DJIA and S\&P 500, respectively. The hypothesis that $\omega=1$ for stocks in both indices is strongly rejected, and there is not sufficient evidence to reject $\omega=0$. On the other hand, the hypotheses that $\gamma=0$ or that $\gamma=1$ are both firmly rejected for non-FIPU stocks in both indices.

To add, estimates of the standard deviation of the measurement equation noise term for abnormal earnings $\sigma_{\varepsilon}$ equal 0.2238 and 0.0251 for the two indices, respectively. These are statistically insignificantly different from zero. Estimates of the standard deviation of the state equation signal shock $\sigma_{\eta}$ equal 0.9818 and 1.9740 . These are strongly different from zero for non-FIPU stocks traded under the DJIA and S\&P 500, respectively.

These results affirm that abnormal earnings, with 'other information' embedded, are mean-reverting. Their persistence and dispersion of the disturbance terms are primarily reflected in $\gamma$ and $\sigma_{\eta}$, respectively. Collectively, these results in essence provide evidence of the usefulness of our technique to extract hidden yet relevant information about abnormal earnings. To add, $\nu_{t}$ for non-FIPU stocks traded under the S\&P 500 mean-reverts at almost twice the rate of $\nu_{t}$ for DJIA stocks since its estimate of $\gamma$ is half that of the other. Therefore, short- to medium-term trading strategies may be more suitable for non-FIPU stocks traded under the S\&P 500. To clarify, assuming that the stock market prices expectations about firm profitability, investors who employ mean reversion trading strategies may find them to be lucrative by setting long (short) positions for stocks, whose abnormal earnings deviate below (above) historical averages for short investment horizons, before rebounding to normal levels. 


\section{Estimates of Abnormal Earnings for FIPU Stocks: SS Method}

For the SS method in Panel B, the persistence parameter for $x_{t}^{a}$, $\omega$ is 0.0025 and 0.0333 with a standard error of 0.0093 and 0.0042 for DJIA and S\&P 500, respectively. In this case, the hypothesis that $\omega=1$ for stocks in both indices is strongly rejected. Furthermore, while there is no sufficient evidence to reject that $\omega=0$ for FIPU blue chip stocks, we do however find that it is statistically significantly different from zero for FIPU stocks traded under the broader index. This indicates a low level of persistence in abnormal earnings for S\&P 500 FIPU stocks.

The persistence parameter for $\nu_{t}, \gamma$ is 0.8302 and 0.9649 with a standard error of 0.0898 and 0.0041 for DJIA and S\&P 500, respectively. The hypotheses that $\gamma=0$ and $\gamma=1$ respectively are both rejected for FIPU stocks traded under both indices at a $10 \%$ significance level or better. To add, there is strong dispersion of both noise and signal shocks for FIPU stocks as quantified by their standard deviation parameters, $\sigma_{\varepsilon}$ and $\sigma_{\eta}$. The variation ranges from 1.25 to 24.84 of which the latter stems from FIPU stocks traded under the S\&P 500. These results are similar to those of Panel A discussed in the previous subsection in that abnormal earnings are again mean-reverting, with their persistence and variation in the disturbance terms largely reflected in $\gamma$ and $\sigma_{\eta}$, respectively. The persistence parameter $\gamma$ values for both FIPU and non-FIPU stocks traded under DJIA are quite comparable to one another; however, $\nu_{t}$ for FIPU stocks traded under the S\&P 500 mean-revert in twice the time of non-FIPU stocks. This suggests that a signal shock to 'other information' today will have long-dated influence on future abnormal earnings for S\&P 500 FIPU firms. Overall, these results again provide evidence of the usefulness of our technique to extract latent information about abnormal earnings, particularly for pricing and forecasting exercises.

Given the contrasting results, it would be of interest to analyze the forecasting ability of abnormal earnings and stock prices using the two estimation techniques, namely, the 
SS method proposed in this paper and the benchmark DHS two-step regression method. We undertake this task in section 3.3.3.

\subsubsection{Forecasts of Stock Prices and Abnormal Earnings}

In this section, inspired by the empirical analyses in Bakshi and Ju (2002) and Bakshi and Chen (2005), we evaluate the in- and out-of-sample pricing and next period $t+1$ abnormal earnings forecasting performance of our state space approach and the benchmark DHS approach on a set of performance metrics.

\section{Metrics Used for Evaluating Forecast Performance}

The procedure for forecasting stock prices with our SS method is as follows. Let the current period be indexed by time $t$. Our objective is to forecast stock prices for period $t$, using all available information to date. This is accomplished by applying Eq. (3.8), for which we need estimates of coefficients $\alpha_{1}$ and $\alpha_{2}$. These are functions of $\omega, \gamma$, and $r$.

The value of the constant discount rate $r$ is chosen as described in Section 3.3.1. In our SS method, estimates of the persistence parameters $\omega$ and $\gamma$ are obtained by ML.

In the SS approach, we need estimates of the latent 'other information' variable $\nu_{t}$ as well, in order to apply Eq. (3.8) to forecast stock prices. Estimates of $\nu_{t}$ are obtained from the Kalman filter recursions. These recursions yield the filter mean of $\nu_{t-1}$, after observing abnormal earnings for period $t x_{t}^{a}$. This is then updated, using Eq. (3.7b), to obtain a predictive mean for $\nu_{t}$. This is used in the pricing Eq. (3.8) to obtain modelimplied stock price for period $t P_{t}$.

In the DHS method, estimates of $\omega_{1}$ and $\gamma_{1}$, obtained from pooled regressions in Eq. (3.12), are used as proxies for $\omega$ and $\gamma$ appearing in Eq. (7). These are used to estimate the coefficients $\alpha_{1}$ and $\alpha_{2}$ in the pricing Eq. (3.8). 
To construct the percentage pricing error and dollar forecast error for abnormal earnings, let $P_{t}$ indicate the actual price at time $t, \hat{P}_{t}$ indicate the model-implied price at time $t$ given by the valuation function in Eq. (3.8), $x_{t+1}^{a}$ indicate the actual next period $t+1$ abnormal earnings, and $E_{t}\left\{x_{t+1}^{a}\right\}$ indicate the conditional expectation of next period $t+1$ abnormal earnings as of time $t$ in Eq. (3.9). Then, the percentage pricing error is $e_{P_{t}}=100 \times\left(P_{t}-\hat{P}_{t}\right) / P_{t}$ and the dollar forecast error for next period $t+1$ abnormal earnings is $e_{x_{t+1}^{a}}=x_{t+1}^{a}-E_{t}\left\{x_{t+1}^{a}\right\}$.

As such, the five standard error criteria used for pricing and abnormal earnings errors include:

$$
\begin{gathered}
M P E / M D E=\frac{\sum_{t=1}^{T} e_{t}}{T} \\
S T D=\sqrt{\frac{1}{T-1} \sum_{t=1}^{T}\left(e_{t}-\bar{e}\right)^{2}} \\
M P S E / M S E=\frac{\sum_{t=1}^{T} e_{t}^{2}}{T} \\
M A P E / M A D E=\frac{\sum_{t=1}^{T}\left|e_{t}\right|}{T} \\
R M P S E / R M S E=\sqrt{\frac{\sum_{t=1}^{T} e_{t}^{2}}{T}}
\end{gathered}
$$

MPE and MDE denote, respectively, the mean percentage pricing error and mean dollar error, and they measure the prediction bias. STD is the standard deviation of the pricing and dollar error time-series, and it measures the variation of the errors over time. The remaining criteria measure prediction accuracy: MAPE and MADE denote the mean absolute percentage error and mean absolute dollar error, MSPE and MSE are the mean square percentage error and mean square error, and RMSPE and RMSE are the root mean square percentage error and root mean square error, respectively. 
Under the assumption that the market prices equities fairly, Bakshi and Chen (2005) suggest that a 'good' forecasting or valuation model, strictly speaking, should have an error $e_{t}$ with a zero mean and low standard deviation over time. The authors further imply that a forecasting or valuation model may still be 'empirically acceptable', even if the mean error is nonzero, as long as $e_{t}$ has negligible variation over time. On one polar end, if the mean and standard deviation of $e_{t}$ are both zero, then the model forecasts perfectly. On the other polar end, if $e_{t}$ is large in magnitude, then the model performance is poor, and it may well be misspecified.

\section{6-2017 In-sample Forecasts}

Since $\nu_{t}$ is unobserved, we need $E_{t}\left(\nu_{t}\right)$ in Eq. (3.8). This is obtained from the SS method, applied to Eqs. (3.7a) and (3.7b), by using the predictive mean for $\nu_{t}$, obtained after observing $x_{t}^{a}$.

Tables 3.4 and 3.6 each report the five in-sample mean pricing and forecasting error measurements as provided above ${ }^{13}$ for our SS method and the benchmark DHS method. Specifically, Table 3.4 reports in-sample results for non-FIPU stocks. Table 3.6 reports in-sample results for FIPU stocks. They also provide the percentage differences for each error measurement of our SS estimation method with respect to that of the benchmark DHS estimation method. We use a 1976-2017 sub-sample to calculate the error measure-

\footnotetext{
${ }^{13}$ We find it important to mention that such standard mean error measurements as calculated in our analysis are slightly troublesome because they do not account for differences in available data points between the two competing estimation methods. For example, after accounting for the two degrees of freedom lost in the data for each stock in the aggregate sample, our SS estimation approach uses a total of 815 and 8,521 data points for the DJIA and S\&P 500 stocks, respectively. Yet, the DHS approach, which is constrained by the availability of analyst forecasts for each stock in the I/B/E/S database, uses only 664 and 7,719 in kind. In our view, such lapse in the number of available data points may over- or under-value the mean error measurements. Specifically, less data available may potentially favor the current mean error calculations for the DHS method relative to our estimation technique. Although we do not adopt one, and, if one exists, a modified mean error calculation that accounts for or penalizes differences in data points would be more appropriate within this context.
} 
ments for both abnormal earnings and stock prices. We do this in order to be consistent with the time-period of data availability for consensus analysts' earnings forecasts. Recall that consensus analysts' next period $t+1$ earnings forecasts are used in order to approximate expected $t+1$ abnormal earnings in the DHS estimation method.

\section{6-2017 In-sample Non-FIPU Model-Implied Prices}

Figure 3.8 provides a graphical demonstration of the model's ability to price stocks under each estimation method relative to actual prices for the 14 firms. The implied residual income valuations utilizing both methods do moderately well for Chevron and DowDuPont but largely undervalue the remaining stocks. Such results do not come as a surprise, though, given the stylized fact in the literature that traditional asset pricing models assuming a constant discount factor do not capture variation in ex-post stock prices very well.

The relative ability of the competing estimation methods to explain contemporaneous stock prices is analyzed in Panel B of Table 3.4. Panel B reports percentage pricing errors for our SS method in the top-half of the panel and the DHS method in the bottomhalf. The MPE ranges from $50.67 \%$ to $54.84 \%$ for both estimation methods indicating that the implied residual income valuation model using either method largely undervalues stocks relative to the aggregate stock market. Although close, the DHS estimation generally demonstrates better valuation ability for non-FIPU stocks relative to our SS method. Specifically, the DHS method values non-FIPU stocks traded under the DJIA and S\&P 500 indices by approximately 2.3 and 4.3 percentage points more accurately than our competing SS approach, respectively. We note here that, although, our SS method exhibits better forecasting performance for abnormal earnings for non-FIPU stocks traded under the S\&P 500 in Panel A (discussed in the next subsection), the corresponding valuation result in Panel B does not. As such, it refutes the notion that improved forecasts 
for abnormal earnings. This result is not necessarily surprising, since explicit forecasts of abnormal earnings are not used in the implied valuation model in Eq. (3.8). The combination of 'other information', persistence parameters, and discount rate determines stock price accuracy. As noted earlier, it is a drawback of our SS approach that we learn about 'other information' $\nu_{t}$ only through the history of abnormal earnings. But Ohlson's model suggests that $\nu_{t}$ is other information that is useful in predicting next period $t+1$ abnormal earnings not contained in current abnormal earnings. Nonetheless, we still yield to the possibility that the valuation model may be misspecified for the broader set of non-FIPU stocks on average under the assumption that the stock market values stocks correctly.

\section{6-2017 In-sample Non-FIPU Forecasts of Next Period Abnormal Earnings}

Figures 3.5 and 3.6 illustrate the in-sample forecasting ability of the DHS and SS estimation methods for abnormal earnings in Eq. (7a) for 14 non-FIPU stocks. Figure 3.7 superimposes the graphical results for each estimation method for complete comparison under one graph. There is ample heterogeneity regarding optimal fit for abnormal earnings between the competing estimation methods across the 14 stocks, with our SS estimation method as the leading candidate for half of those stocks. For example, the in-sample predictions of our SS estimation method are clearly superior in fit for Verizon, DowDuPont, Merck \& Co, United Technologies, Walmart, Microsoft, and Cisco Systems compared to those of the benchmark DHS method. Both methods, at least visually, are fairly comparable to one another for the remaining 7 stocks. We quantify the performance evaluation for the full array of 216 non-FIPU stocks in the sample.

Panel A of Table 3.4 provides dollar forecast errors for abnormal earnings for our SS method in the top-half of the panel and the DHS method in the bottom-half. For the SS estimation method, the mean dollar error for abnormal earnings is 0.1184 and 0.1345 for 
DJIA and S\&P 500 stocks, respectively. This result suggests that, on average between 1976-2017, forecasts on next period $t+1$ abnormal earnings using our SS estimation method are roughly 12-13 cents per share below realized $t+1$ abnormal earnings for both blue chip and broad index stocks. In contrast, for the DHS estimation method, the mean error for abnormal earnings is -0.0973 and -0.2273 for DJIA and S\&P 500 stocks, respectively. This result reflects the wide-ranging over-optimism in analysts' forecasts in earnings across firms.

The SS and DHS methods both clearly indicate prediction bias for abnormal earnings. The former has a $28 \%$ smaller standard deviation in the bias for the S\&P 500 stocks at $27.31 \%$ compared to the latter at almost $38 \%$. The latter has a $5.5 \%$ smaller standard deviation in the bias for the DJIA stocks at $30.2 \%$ compared to the former at almost $32 \%$. The measures of forecast accuracy indicate that the SS estimation method has superior predictive ability for abnormal earnings for non-FIPU stocks traded under the S\&P 500, while the competing DHS method does a better job for non-FIPU blue chip stocks. This result provides further support for using a state-space approach to predict next period $t+1$ abnormal earnings for a broader set of stocks. Yet, it also reiterates the important role of 'other information' embedded in analysts' forecasts in predicting next period $t+1$ abnormal earnings, at least for blue chip stocks.

\section{6-2017 In-sample FIPU Model-Implied Prices}

Figure 3.10 provides a set of graphs illustrating the model's ability to price stocks, under each estimation method, relative to actual prices, for the 10 firms. The implied residual income valuations, utilizing both methods, again largely undervalue stocks, with valuations for J.P. Morgan Chase and Bank of America being the most promising. The relative ability of the competing estimation methods to explain contemporaneous stock prices in the sample is evaluated in Panel B of Table 3.6. Panel B reports the percent- 
age pricing errors for our SS method in the top-half of the panel and the DHS method in the bottom-half. Similar to non-FIPU stocks, the MPE for both estimation methods indicates sizable undervaluation of FIPU stocks relative to the aggregate stock market. One can readily see that the DHS estimation demonstrates superior valuation precision for FIPU stocks relative to our SS method by 7 and 17.6 percentage points for the DJIA and S\&P 500, respectively. In addition to the in-sample pricing results for the 1976-2017 sub-sample period in Panel B of Table 3.4, this provides evidence for the superior ability of the benchmark two-step regression employed in DHS to value firms within the financial, insurance, and public utility sectors relative to our SS approach. The valuation precision for the DHS method relative to our SS method is better by 2 percentage points on average for blue chip stocks. Yet, it is definitively better by 30 percentage points on average for S\&P 500 stocks.

\section{In-sample FIPU Forecasts of Next Period Abnormal Earnings}

Figure 3.9 depicts the in-sample forecasting ability of both SS and DHS estimation methods for abnormal earnings for 10 FIPU stocks. In this case, it is readily clear that the SS method is the optimal predictor of in-sample abnormal earnings for virtually all of them. As done for the non-FIPU stocks, we quantify the performance analysis to the full set of 70 FIPU stocks in the sample.

Table 3.6 reports the five performance yardsticks for both estimation approaches as in Table 3.4. Panel A of Table 3.6 provides dollar errors for abnormal earnings for our SS method in the top-half of the panel and the DHS method in the bottom-half. For the SS estimation method, the MDE for abnormal earnings is 0.1234 and -0.0257 for DJIA and S\&P 500 stocks, respectively. The under-prediction (about 12 cents) of our SS approach for FIPU stocks traded under the DJIA is in line with that of non-FIPU stocks. However, we find a 2.5 cent MDE for FIPU stocks traded under the S\&P 500, indicating 
that our SS method is slightly over-optimistic on average. On the other hand, similar to non-FIPU stocks, the DHS estimation method continues to forecast substantially above realized $t+1$ abnormal earnings where the MDE is -0.2098 and -0.7307 for DJIA and S\&P 500 stocks, respectively. This result reaffirms the significant over-optimism in analysts' earnings forecasts, noticeably for FIPU stocks.

The three measures of forecast accuracy of abnormal earnings for FIPU blue chip stocks collectively indicate that the SS estimation method is more accurate than the DHS estimation method on average by about 3\%. So far, within the context of forecasting next period $t+1$ abnormal earnings for the 1976-2017 sub-sample period, our SS method seems to outperform the DHS method for FIPU and non-FIPU stocks traded under the DJIA and S\&P 500, respectively.

It is a drawback of our SS approach adopted here that we learn about $\nu_{t}$ in Eq. (7) only through the observed history of $x_{t}^{a}$. But, Ohlson's (1995) model suggests that $\nu_{t}$ is 'other information' useful in predicting next period $x_{t+1}^{a}$ that is not contained in current period $x_{t}^{a}$.

Therefore, it would be worth learning about $\nu_{t}$ from an expanded set of variables, such has capital markets-specific, firm-specific, and macroeconomic data. This is likely to improve the performance of the SS approach further. We leave this task for future research.

\section{0-2017 In-sample Forecasts}

It is important to recall that one of the empirical deficiencies of the DHS estimation method is the dependence on analysts' forecasts needed to estimate $\nu_{t}$ in order to value contemporaneous stock prices. Such analyst forecast data in the I/B/E/S database were not widely available across stocks until 1976. As such the first 26 years of the primary 1950-2017 sample period is unaccounted for in the pricing and forecasting error analysis. 
By way of contrast, our SS estimation method does not require analyst forecast data in order to generate either ex ante or ex post 'other information' $\nu_{t}$.

Tables 3.5 and 3.7 each display the same five performance metrics for our SS method and the benchmark DHS method only now for the 1950-2017 full sample period. Specifically, Table 3.5 reports 1950-2017 in-sample results for non-FIPU stocks. Table 3.7 reports 1950-2017 in-sample results for FIPU stocks. Panels A and B for each table report the dollar and percentage pricing error estimates for abnormal earnings and stock prices, respectively. Note the error estimates remain the same as in Tables 3.4 and 3.6 for the DHS method.

\section{0-2017 In-sample Non-FIPU Model-Implied Prices}

For the SS estimation method in Panel B of Table 3.5, the MPE is $51.05 \%$ and $47.67 \%$ for non-FIPU stocks traded under the DJIA and S\&P 500 indices, respectively. These estimates are substantial improvements in the implied valuation model based on our SS method for the full 1950-2017 sample period compared to those in Panel B of Table 3.4. The valuation model using the SS method reduces the undervaluation problem by approximately 5 percentage points in the full sample. Valuation for non-FIPU stocks traded under the S\&P 500 shows the largest valuation improvement by 7 percentage points albeit with a larger STD. To support, in contrast to Panel B of Table 3.4, the measures of pricing accuracy now show that the implied valuation model based on our SS estimation approach is the favorable one.

\section{0-2017 In-sample Non-FIPU Forecasts of Next Period Abnormal Earnings}

For the SS estimation method in Panel A of Table 3.5, the MDE (STD) for abnormal earnings is 0.0786 and 0.0869 (0.259 and 0.2262$)$ for DJIA and S\&P 500 stocks, respectively. Compared to the MDE for the SS method in Panel A of Table 3.4, this result demonstrates 
a forecast improvement of roughly 4-5 cents from 12-13 cents to 8-9 cents below realized abnormal earnings per share on average across stocks. The STD also reduces in value by nearly 5-6 cents demonstrating lower volatility of the errors.

The remaining measures of forecast accuracy further illustrate the forecast improvement of our SS method using the full sample period of abnormal earnings for non-FIPU stocks. In fact, our SS method now has about $20 \%$ more accurate forecasts than those of the benchmark DHS method for non-FIPU stocks traded under the DJIA, compared to the results in the previous table.

\section{0-2017 In-sample FIPU Model-Implied Prices}

For the SS estimation method in Panel B of Table 3.7, it reduces the undervaluation problem by 3-4 percentage points for FIPU stocks and consequently demonstrates a smaller MPE or undervaluation relative to the DHS method, along with a smaller STD

for S\&P 500 FIPU stocks. Using the measures of forecast accuracy within this context, the valuation model based on our SS method forecasts best for FIPU blue chip stocks, while one based on the competing DHS method predicts best for FIPU stocks traded under the broader S\&P 500 index.

\section{0-2017 In-sample FIPU Forecasts of Next Period Abnormal Earnings}

In Panel A of Table 3.7, we find that the prediction bias as measured by the MDE of abnormal earnings for FIPU stocks is incrementally reduced for our SS method by 13 and 20\% under the DJIA and S\&P 500 indices, respectively. The DHS method is still the leading method for prediction precision of next period $t+1$ abnormal earnings for FIPU stocks traded under the S\&P 500 as in Table 3.6, while our SS method is best for FIPU blue chip stocks. 


\section{Further Analysis of In-sample Model-Implied Price Performance}

We additionally examine relative valuation performance of each estimation method for the full 1950-2017 sample from a simple regression specification:

$$
P_{t}=\beta_{0}+\beta_{1} \hat{P}_{t}+\tilde{\eta}_{t}
$$

where $P_{t}$ is the market price in year $t$ and $\hat{P}_{t}$ is the corresponding model price determined by the estimation method under consideration. If the valuation model under a given estimation method immaculately fits market stock price variation over time, then $\beta_{1}=1$ and the regression $R^{2}=100 \%$.

Panel A of Table 3.8 reports the regression results for each estimation method for non-FIPU stocks. Panel B does the same for FIPU stocks.

For the valuation performance of non-FIPU blue chip stocks, the $R^{2}$ for both estimation methods are practically equal to each other, with the $\beta_{1}$ coefficient for our SS method closer to 1 . This result is in line with the performance metrics in Panel B of Table 3.5, yielding a more accurate valuation under the SS estimation method.

In contrast, the $R^{2}$ and $\beta_{1}$ coefficient for the DHS benchmark method is uniformly better than our SS method for non-FIPU stocks traded under the S\&P 500. This contrasts with the results in Panel B of Table 3.5 for S\&P 500 non-FIPU stocks. It is worth mentioning that, relative to non-FIPU blue chips, the lower $R^{2}$ for non-FIPU stocks traded under S\&P 500 for both estimation methods potentially implies that systematic factors unaccounted for in the residual income valuation model may be critical in explaining stock market prices.

For FIPU stocks, in this case, the DHS method unreservedly produces a better fitting valuation model, especially for FIPU stocks traded under the S\&P 500. For the DHS method, the $R^{2}$ is about 66 percentage points higher than the SS method for S\&P 500 
FIPU stocks. In addition, the $\beta_{1}$ coefficient for our SS method is not statistically different from zero which implies that the valuation model under this estimation method is severely misspecified for a broader set of FIPU stocks. These results are not surprising and are comparable to those in Panel B of Table 3.7.

Given that the in-sample performance yardsticks and regression results in Tables 3.4-3.8 show mixed results contingent upon whether we use the full sample or restricted sample, we next conduct out-of-sample stock price valuation under both sample types.

\section{Out-of-sample Forecasts}

For out-of-sample pricing under each estimation method, we take as input the parameter values estimated from the 3 years prior to and including year $t$ and apply them to the valuation formula in Eq. (3.8) for year $t$ to determine the model-implied price for each stock. Next, we proceed to year $t+1$ to get a new trailing 3-year sub-sample and apply the re-estimated parameters to year $t+1$. For example, to determine a model price for IBM in year 2000, one would use IBM data from 1998-2000 to calculate parameter estimates to apply to the valuation formula for IBM in year 2000. Then, roll over by one-period to re-estimate parameters using 1999-2001 data to compute a model price for IBM in year 2001. This rolling-estimation procedure continues until 2017. In our analysis, because we lose two degrees of freedom in the time-series for each stock (see footnotes 3 and 4), we start the valuation exercise no earlier than the year 1952. The initial 3 years of the 1952-2017 data sample are required to determine the first set of out-of-sample pricing errors. Thus, the out-of-sample pricing results are based on the 1954-2017 valuation periods. Consequently, there are at most 64 observations of pricing errors for any stock.

Additionally, we conduct a 5-year rolling-sample estimation procedure for robustness. We find it important to mention that given the time-series of the data is short and 
the data frequency is in annual intervals, we face a hurdle of bias and large variance for the rolling-sample parameter estimates under both competing estimation methods. A longer time-series and/or shorter intervals (e.g. quarterly or monthly data) would alleviate the problem statistically.

Considering that the rolling-estimation procedure is computationally-intensive, we administer the out-of-sample pricing evaluation to an arbitrarily chosen set of 24 stocks (14 non-FIPU stocks and 10 FIPU stocks): American Express, Aon, Apple, Bank of America, Chevron, Cisco Systems, DowDuPont, Duke Energy, ExxonMobil, Goldman Sachs, Home Depot, IBM, Intel, JPM Chase, Merck \& Co, Microsoft, Nike, Nisource, Pinnacle, United Tech., UnitedHealth, Verizon, Visa, and Walmart.

As noted in Bakshi and Chen (2005), since the out-of-sample valuation for each stock is based on the parameters estimated from each stock's recent 3-year or 5-year history, the model valuation implies what the stock price should be today if the market priced the stock under the residual income framework as in the past 3 or 5 years. In other words, the model price reflects the market's recent valuation standard for the stock.

\section{Out-of-sample Model-Implied Price Performance}

Tables 3.9 and 3.10 report the out-of-sample pricing performance metrics of the 3year and 5-year rolling sub-samples for the 1976-2017 valuation period respectively, as done for the in-sample counterpart. ${ }^{14}$ Panel A in each table displays the consolidated results for the 14 non-FIPU stocks and Panel B for the 10 FIPU stocks. There are three main features of the rolling-window performance results. First, the valuation model under our SS estimation approach generally performs better across the 24 stocks than the competing DHS estimation method. Second, the out-of-sample results for the SS approach are

\footnotetext{
${ }^{14}$ We exclude results of the 1954-2017 full valuation period, given similarities to those of the 1976-2017 sub-sample valuation period.
} 
comparable between the 3- and 5-year rolling sub-sample performance metrics. Third, both 3- and 5-year rolling-estimation results for the SS approach in terms of valuation bias (MPE) and valuation accuracy (MAPE, MSPE, and RMSPE) are substantially better than their in-sample equivalents, but typically with larger variation (STD). For example, the valuation bias for the out-of-sample results ranges from 16-30\%, compared to that of its in-sample counterpart of $31-55 \%$. Similarly, the valuation accuracy metrics for the out-of-sample results range from 18-59\%, compared to those of the in-sample results of $20-74 \%$. However, the STD for the out-of-sample results for non-FIPU (FIPU) stocks range from $31-47 \%$ (41-56\%), compared to those of the in-sample results of 16.5$17 \%(32.5-65 \%)$. Such larger variation in the out-of-sample errors both over time and across stocks motivates an in-depth analysis of their properties discussed later in this section.

Result three above is surprising. At current period time $t$, having observed abnormal earnings $x_{t}^{a}$, SS method forms a prediction for the 'other information' variable $\nu_{t}$. This prediction is used in the price valuation formula given in Eq. (3.8). Price formula is a function of the estimated parameters, $\omega$ and $\gamma$, as well as $\nu_{t}$. Result three suggests that using only the most recent data presumably leads to more accurate estimates of $\omega$ and $\gamma$, as well as of $\nu_{t}$.

For the benchmark DHS method, on the other hand, its rolling-estimation out-ofsample results are generally worse than their respective in-sample results. Also, surprisingly, the 5-year rolling sub-sample results for FIPU stocks are decidedly worse than those in the 3-year rolling sub-sample. In fact, the valuation accuracy of the 3-year results for the DHS estimation method are on par with those of our SS method, with a lower MPE equal to 5.7\% (albeit a larger STD equal to 62.5\%). Yet, they deviate substantially for the 5-year rolling sub-sample, where MPE is $-55.33 \%$, STD is over $300 \%$, and the smallest (largest) valuation accuracy criterion MAPE (MSPE) is nearly $100 \%(1000 \%)$. 
Figures 3.11-3.14 illustrate the valuation horse-race between the SS and DHS estimation methods for the 3- and 5-year rolling sub-samples of the 24 stocks. The rollingwindow valuation under our SS estimation approach track stock prices moderately better than the 5-year. The opposite is true for the DHS approach, especially for FIPU stocks, like Aon and Bank of America.

\section{Out-of-sample Forecast Errors}

In light of the large STD of the model mispricing previously discussed, we now analyze their patterns and their properties. Since the relative out-of-sample performance for our SS estimation approach typically does a better job pricing stocks than the competing DHS benchmark as mentioned above, we concentrate on mispricing under our SS approach only.

Figures 3.15 and 3.16 add the percentage pricing-error path along with the actual and model price paths for each stock for both the 3- and 5-year rolling sub-samples. One can readily see that, for most stocks, especially FIPU stocks, pricing errors have trend and are highly volatile. The large volatility of the percentage errors is expected given the short time-series and low frequency of the data. However, some stocks are particularly mispriced with a trending pattern, such as Verizon, ExxonMobil, Microsoft, Walmart, and all of the FIPU stocks (Visa has too small of a sample much less a rolling sub-sample to make a reasonable inference). Except for a few outliers, the percentage pricing errors for Chevron, Intel, and United Technologies are relatively small and stable over time, especially for the 5-year rolling window. Nonetheless, the fact that the model pricing errors would systematically go through periods of high and low levels (especially for FIPU stocks) suggests that other firm-specific or macroeconomic factors may be important for the market's valuation. 


\section{Further Analysis of Out-of-sample Forecast Errors}

To further understand the mispricing of the model under our estimation approach, we look at both the autocorrelation and cross-stock correlations of the out-of-sample pricing errors. Tables 3.11 and 3.12 show the autocorrelations at lags up to 5 years for the 3- and 5-year rolling sub-samples, respectively. Panel A in each table provides autocorrelations for non-FIPU stocks and Panel B for FIPU stocks. In Table 3.11, at the 1-year lag, the autocorrelations for non-FIPU stocks (FIPU stocks) range from -0.04 to $0.61(-0.15$ to 0.53$)$. At the 2-year lag, they drop for each stock. Some stocks see further reduction in autocorrelations, while most (particularly FIPU stocks) see increases at the 3-year lag. But, more firms in the sample start to have negative autocorrelations by the 5-year lag.

Since we have performed both 3- and 5-year rolling sub-sample valuation exercises, we use the Ljung-Box Q-test to test for the absence of autocorrelation at the 3- and 5-year lags for each rolling sub-sample. For the 3-year rolling-window in Table 3.11, the $\mathrm{Q}(3)$ and $\mathrm{Q}(5)$-statistics with their respective p-values for ExxonMobil, Microsoft, JPM Chase, Aon, and Duke Energy suggest that the null hypothesis for the absence of autocorrelation is clearly rejected.

For the 5-year rolling sub-sample in Table 3.12, the autocorrelations at the 1-year lag for non-FIPU stocks (FIPU stocks) are higher than their counterparts in Table 3.11, ranging from 0.03 to 0.71 (-0.85 to 0.70$)$. The autocorrelations also decrease at the 2-year lag similar to Table 3.11. As the lag increases to 3 years and 5 years, more firms see continued decrease in autocorrelations.

For the 5-year rolling-window in Table 3.12, the $\mathrm{Q}(3)$ and $\mathrm{Q}(5)$-statistics with their respective p-values for Chevron, ExxonMobil, Home Depot, IBM, Merck \& Co, Nike, Walmart Pinnacle, American Express, JPM Chase, Aon, Bank of America, UnitedHealth, and Visa emphatically reject the null hypothesis of no autocorrelation. The pricing er- 
rors for ExxonMobil, JPM Chase, and Aon prove to be highly persistent regardless of the rolling sub-sample. Altogether, the percentage pricing errors are persistent within 3-5 years, suggesting that firm-specific and/or macroeconomic factors are relevant in pricing these firms.

Tables 3.13 and 3.14 provide the degree of covariation in the percentage pricing errors across stocks under the following simple time-series regression:

$$
\varepsilon_{i, t}=a_{i}+b_{i} \varepsilon_{i, t}^{*}+\tilde{\omega}_{i, t}, \quad i=1, \ldots, I
$$

where $\varepsilon_{i, t}$ is the percentage pricing error of stock $i$ in year $t$ and $\varepsilon_{i, t}^{*}$ is the percentage pricing error of IBM and UnitedHealth (also arbitrarily chosen for comparison) for nonFIPU and FIPU stocks, respectively. Each table reports the b coefficient and its standard error as a correlation indicator between $i$ stock and its respective benchmark stock, IBM or UnitedHealth, along with its associated t-statistic and adjusted $R^{2}$. For most of the stocks, the b estimates are insignificant, with negative adjusted $R^{2}$ values indicating that cross-correlations of the percentage pricing errors overall are non-existent. For the 3year rolling sub-sample, Cisco Systems has a statistically insignificant b coefficient but with the highest adjusted $R^{2}$ of $12.50 \%$ for non-FIPU stocks. In the 5-year counterpart, both Intel and Duke Energy also have statistically insignificant b coefficients but both with the highest adjusted $R^{2}$ values of $14.18 \%$ and $27.64 \%$, respectively. Such high $R^{2}$ values make sense for both Cisco Systems and Intel relative to IBM, as they all operate in the information technology sector. Duke Energy relative to UnitedHealth is an unexpected result. Visa severely suffers from small sample bias (only a maximum of 6 time-observations for the valuation exercise), therefore its adjusted $R^{2}$ is superficially exacerbated. 
These results on pricing-error persistence and correlations potentially imply that there are most-likely time-varying factors either unique to the firm or common in the macroeconomy that may be relevant to the model.

\section{Summary of Empirical Results}

The parameter estimates under our SS estimation approach for abnormal earnings reported in Table 3.3 and discussed in section 3.2 above indicate that abnormal earnings, with 'other information' embedded, are mean-reverting and their strongly significant persistence and variation in the disturbance terms are primarily reflected in the $\gamma$ and $\sigma_{\eta}$ parameters respectively. In this context, abnormal earnings for FIPU stocks mean revert twice as fast as non-FIPU stocks traded under the broader S\&P 500 index. Under the benchmark DHS method, the parameter estimates also indicate that abnormal earnings mean revert. Strong persistence and dispersion of disturbance terms are primarily reflected in $\omega$ and $\sigma_{\varepsilon}$ for non-FIPU stocks and $\gamma$ and $\sigma_{\eta}$ for FIPU stocks.

We conduct in- and out-of-sample forecast performance analysis to determine a performance leader. Figures 3.5-3.10 graphically illustrate the heterogeneity in the insample pricing and forecasting performances of both estimation methods for 24 stocks. However, Figures 3.11-3.14 depict that our SS estimation approach generally does a better job pricing the 24 stocks in both 3- and 5-year rolling-windows. We quantify the figures by reporting performance yardsticks in Tables 3.4-3.10. The in-sample exercises produce mixed results, contingent upon using either the restricted 1976-2017 sub-sample or the 1950-2017 full sample. Yet, we find that our SS approach is generally favorable in valuing both FIPU and non-FIPU stocks out-of-sample. Finally, we evaluate the crosscorrelation and time-series properties of the percentage pricing errors in Tables 3.11-3.14 and Figures 3.15 and 3.16. The pricing errors in the figures exhibit trend and high volatility, especially for FIPU stocks, indicating both persistence over time and large mispricing 
for specific years, respectively. There is high persistence in the pricing errors for some firms, including ExxonMobil, JPM Chase, and Aon for 3-5 years. In contrast, we find little evidence of cross-correlation between stocks. Overall, we infer that there is at least time-varying firm-relevant or market-wide factors omitted from the valuation model.

\subsection{Conclusions}

We assess the empirical implications of the residual income valuation model for equity prices developed in Ohlson (1995) by accounting for residual income information dynamics. A key assumption of the Ohlson (1995) residual income model stipulates that next period $t+1$ residual income is a linear function of current period $t$ residual income and a latent variable referred to as 'other information'. This 'other information' contains information on next period $t+1$ abnormal earnings not reflected in current period $t$ abnormal earnings. Previous work in Dechow et al. (1999) is the first to explicitly account for this variable in empirical application of the residual income model. They proxy this 'other information' variable with consensus analysts' forecasts of earnings. In this paper, we propose to estimate this latent 'other information' variable using a state space framework instead. Our method obviates the need for analysts' earnings forecasts.

Persistence and dispersion of the disturbance terms for abnormal earnings are primarily reflected in the parameters of the 'other information' variable $\nu_{t}$ using our SS estimation approach. This signifies the importance of the latent variable in predicting next period $t+1$ abnormal earnings. It also demonstrates the usefulness of our approach to extract hidden yet relevant information about abnormal earnings. Cognizant of the flawed two-step regression benchmark approach in DHS, persistence and dispersion of the disturbance terms for abnormal earnings are reflected in the parameters of both current abnormal earnings and 'other information'. 
Empirical results indicate that our SS estimation approach is promising in pricing stocks and predicting next period $t+1$ abnormal earnings, relative to the two-step regression benchmark approach in DHS. The SS estimation approach leads the DHS counterpart for both 3- and 5-year rolling-window out-of-sample stock pricing.

Examination of the time-series properties of out-of-sample pricing errors across stocks demonstrate high persistence and large mispricing for some years, prominently for FIPU firms. We find no direct evidence of cross-correlation in pricing errors. Given the time trend and large volatility of pricing errors, we deduce that time-varying firm-specific and/or market-wide factors are omitted variables in the residual income valuation model.

The empirical results suggest at least a few research directions. First, one can introduce stochastic discount rates in our state space application that would lead to a richer valuation model. Second, it may be empirically desirable to examine jump-diffusion processes for abnormal earnings, especially for firms in the FIPU sectors, given the nonGaussian nature of the data. Lastly, one can consider extracting the 'other information' variable using a rich set of data on accounting, capital market, and macroeconomic variables. We leave this last task for future work. 
Table 3.1: Summary Statistics: Non-FIPU Firms

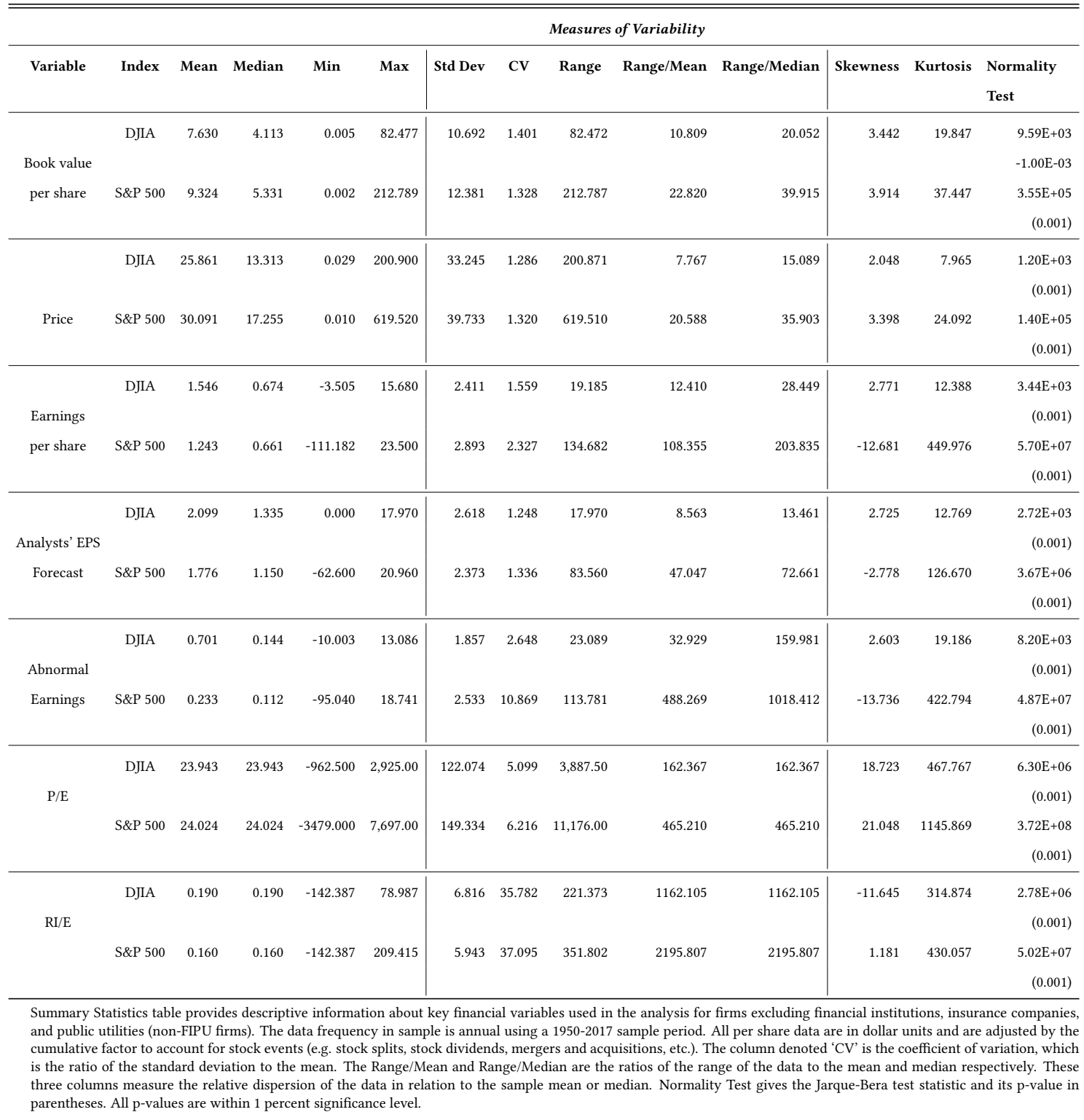


Table 3.2: Summary Statistics: FIPU Firms

\begin{tabular}{|c|c|c|c|c|c|c|c|c|c|c|c|c|c|}
\hline \multirow{3}{*}{ Variable } & \multirow{3}{*}{ Index } & \multicolumn{12}{|c|}{ Measures of Variability } \\
\hline & & Mean & Median & Min & Max & Std Dev & $\mathrm{CV}$ & Range & Range/Mean & Range/Median & Skewness & Kurtosis & Normality \\
\hline & & & & & & & & & & & & & Test \\
\hline \multirow{5}{*}{$\begin{array}{l}\text { Book value } \\
\text { per share }\end{array}$} & DJIA & 24.413 & 11.767 & 0.011 & 182.474 & 36.414 & 1.492 & 182.463 & 7.474 & 15.507 & 2.771 & 10.621 & $5.85 \mathrm{E}+02$ \\
\hline & & & & & & & & & & & & & $-1.00 \mathrm{E}-03$ \\
\hline & S\&P 500 & 23.451 & 12.800 & 0.007 & 781.772 & 44.349 & 1.891 & 781.765 & 33.336 & 61.075 & 9.095 & 123.608 & $1.41 \mathrm{E}+06$ \\
\hline & & & & & & & & & & & & & $(0.001)$ \\
\hline & DJIA & 44.670 & 29.420 & 0.113 & 254.760 & 52.892 & 1.184 & 254.647 & 5.701 & 8.656 & 1.921 & 6.647 & $1.85 \mathrm{E}+02$ \\
\hline \multirow{3}{*}{ Price } & & & & & & & & & & & & & $(0.001)$ \\
\hline & S\&P 500 & 42.732 & 23.525 & 0.023 & 1971.250 & 104.774 & 2.452 & 1971.227 & 46.130 & 83.793 & 10.811 & 147.651 & $2.02 \mathrm{E}+06$ \\
\hline & & & & & & & & & & & & & $(0.001)$ \\
\hline \multirow{4}{*}{$\begin{array}{l}\text { Earnings } \\
\text { per share }\end{array}$} & DJIA & 3.162 & 1.858 & -5.560 & 26.340 & 4.479 & 1.416 & 31.900 & 10.088 & 17.171 & 2.686 & 11.709 & $6.89 \mathrm{E}+02$ \\
\hline & & & & & & & & & & & & & $(0.001)$ \\
\hline & S\&P 500 & 2.191 & 1.443 & -756.800 & 107.600 & 16.928 & 7.726 & 864.400 & 394.533 & 599.237 & -39.467 & 1783.534 & $3.00 \mathrm{E}+08$ \\
\hline & & & & & & & & & & & & & $(0.001)$ \\
\hline \multirow[b]{2}{*}{ Analysts' EPS } & DJIA & 3.641 & 2.350 & 0.010 & 22.630 & 4.365 & 1.199 & 22.620 & 6.213 & 9.626 & 2.341 & 8.417 & $3.05 \mathrm{E}+02$ \\
\hline & & & & & & & & & & & & & $(0.001)$ \\
\hline \multirow[t]{3}{*}{ Forecast } & S\&P 500 & 3.329 & 2.030 & -3.300 & 132.080 & 7.325 & 2.200 & 135.380 & 40.666 & 66.690 & 10.769 & 152.184 & $1.89 \mathrm{E}+06$ \\
\hline & & & & & & & & & & & & & $(0.001)$ \\
\hline & DJIA & 0.454 & 0.221 & -12.777 & 17.626 & 3.007 & 6.629 & 30.402 & 67.014 & 137.609 & 1.098 & 16.199 & $1.14 \mathrm{E}+03$ \\
\hline Abnormal & & & & & & & & & & & & & $(0.001)$ \\
\hline \multirow[t]{2}{*}{ Earnings } & S\&P 500 & -0.479 & 0.086 & -847.693 & 27.820 & 18.406 & 38.425 & 875.513 & 1827.748 & 10184.832 & -44.428 & 2042.545 & $3.82 \mathrm{E}+08$ \\
\hline & & & & & & & & & & & & & $(0.001)$ \\
\hline \multirow{4}{*}{$\mathrm{P} / \mathrm{E}$} & DJIA & 17.126 & 17.126 & -38.449 & 193.182 & 17.977 & 1.050 & 231.630 & 13.525 & 13.525 & 6.023 & 60.162 & $2.25 \mathrm{E}+04$ \\
\hline & & & & & & & & & & & & & $(0.001)$ \\
\hline & S\&P 500 & 18.129 & 18.129 & -467.188 & 2355.000 & 83.547 & 4.608 & 2822.188 & 155.673 & 155.673 & 21.507 & 569.261 & $3.05 \mathrm{E}+07$ \\
\hline & & & & & & & & & & & & & $(0.001)$ \\
\hline \multirow{4}{*}{$\mathrm{RI} / \mathrm{E}$} & DJIA & -0.040 & -0.040 & -34.842 & 3.542 & 2.919 & 73.862 & 38.384 & 971.246 & 971.246 & -11.194 & 133.857 & $1.12 \mathrm{E}+05$ \\
\hline & & & & & & & & & & & & & $(0.001)$ \\
\hline & S\&P 500 & -0.172 & -0.172 & -250.879 & 64.208 & 8.147 & 47.357 & 315.086 & 1831.601 & 1831.601 & -23.638 & 694.209 & $4.40 \mathrm{E}+07$ \\
\hline & & & & & & & & & & & & & $(0.001)$ \\
\hline
\end{tabular}


Table 3.3: Parameter Estimates

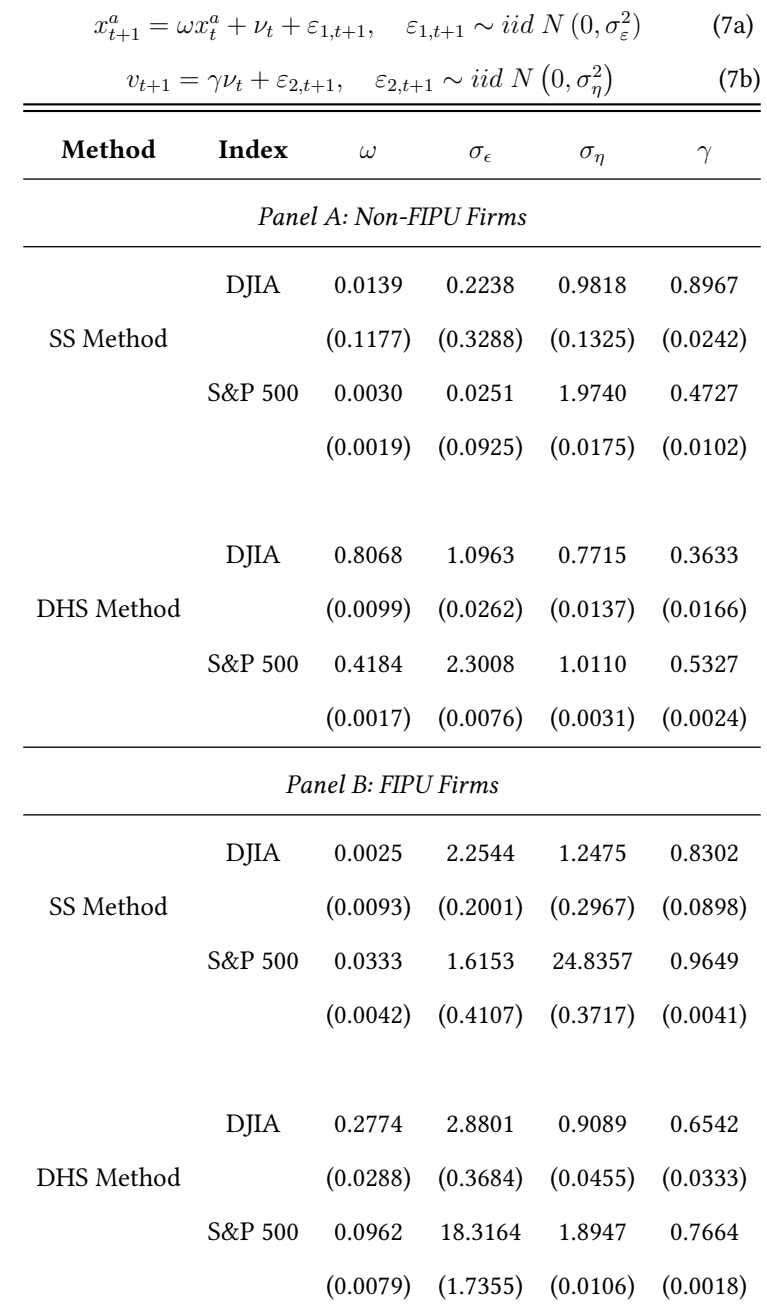

Maximum likelihood parameter estimates for Ohlson's dynamic linear information model for abnormal earnings in Eqs. (7a) and (7b) are reported in Panel A for firms excluding financial institutions, insurance companies, and public utilities (non-FIPU firms). The same parameter estimates are reported in Panel B for financial institutions, insurance companies, and public utilities (FIPU firms). The SS Method calculates parameter estimates based on our state space application via the Kalman filtering recursive procedure. The DHS Method calculates parameter estimates based on two unconditional pooled regressions of Eqs. (7a) and (7b). Numbers in parentheses for parameter estimates are their standard errors. 
Table 3.4: 1976-2017 In-sample Pricing and Forecast Errors: Non-FIPU Firms

\begin{tabular}{|c|c|c|c|c|c|c|c|c|c|c|c|c|}
\hline \multirow[b]{2}{*}{ Method } & \multirow[b]{2}{*}{ Variable } & \multirow[b]{2}{*}{ Index } & \multicolumn{6}{|c|}{$\begin{array}{c}\text { 1976-2017 Sub-sample } \\
\text { Non-FIPU Firms }\end{array}$} & \multirow[b]{2}{*}{ MSE } & \multirow[b]{2}{*}{$\%$ diff } & \multirow[b]{2}{*}{ RMSE } & \multirow[b]{2}{*}{$\%$ diff } \\
\hline & & & MDE & $\%$ diff & STD & $\%$ diff & MADE & $\%$ diff & & & & \\
\hline \multicolumn{13}{|c|}{ Panel A: Abnormal Earnings } \\
\hline \multirow{3}{*}{ SS Method } & & DJIA & 0.1184 & $-221.74 \%$ & 0.3185 & $5.48 \%$ & 0.2557 & $18.36 \%$ & 0.1131 & $14.82 \%$ & 0.3363 & $7.15 \%$ \\
\hline & $x_{t}^{a}$ & & & & & & & & & & & \\
\hline & & S\&P 500 & 0.1345 & $-159.15 \%$ & 0.2731 & $-27.72 \%$ & 0.2090 & $-16.27 \%$ & 0.0909 & $-52.42 \%$ & 0.3015 & $-31.02 \%$ \\
\hline \multirow{3}{*}{ DHS Method } & & DJIA & -0.0973 & - & 0.3020 & - & 0.2161 & - & 0.0985 & - & 0.3138 & - \\
\hline & $x_{t}^{a}$ & & & & & & & & & & & \\
\hline & & S\&P 500 & -0.2273 & - & 0.3779 & - & 0.2496 & - & 0.1911 & - & 0.4371 & - \\
\hline Method & Variable & Index & MPE & $\%$ pt diff & STD & $\%$ pt diff & MAPE & $\%$ pt diff & MSPE & $\%$ pt diff & RMSPE & $\%$ pt diff \\
\hline \multicolumn{13}{|c|}{ Panel B: Stock Price } \\
\hline \multirow{3}{*}{ SS Method } & & DJIA & $54.09 \%$ & $1.56 \%$ & $17.18 \%$ & $3.58 \%$ & $54.09 \%$ & $1.56 \%$ & $32.14 \%$ & $2.74 \%$ & $56.69 \%$ & $2.47 \%$ \\
\hline & $P_{t}$ & & & & & & & & & & & \\
\hline & & S\&P 500 & $54.84 \%$ & $4.17 \%$ & $16.52 \%$ & $0.38 \%$ & $54.84 \%$ & $4.17 \%$ & $32.74 \%$ & $4.53 \%$ & $57.22 \%$ & $4.10 \%$ \\
\hline \multirow{3}{*}{ DHS Method } & & DJIA & $52.53 \%$ & - & $13.60 \%$ & - & $52.53 \%$ & - & $29.40 \%$ & - & $54.22 \%$ & - \\
\hline & $P_{t}$ & & & & & & & & & & & \\
\hline & & S\&P 500 & $50.67 \%$ & - & $16.14 \%$ & - & $50.67 \%$ & - & $28.22 \%$ & - & $53.12 \%$ & - \\
\hline
\end{tabular}

In-sample pricing and forecast errors table provides five error measurements for firms excluding financial institutions, insurance companies, and public utilities (non-FIPU firms) for the 1976-2017 sub-sample period: Mean Dollar Error (MDE) or Mean Percentage Pricing Error (MPE), Standard Deviation (STD), Mean Absolute Dollar Error (MADE) or Mean Absolute Percentage Error (MAPE), Mean Squared Error (MSE) or Mean Squared Percentage Error (MSPE), and Root Mean Squared Error (RMSE) or Root Mean Squared Percentage Error (RMSPE). Panel A reports each dollar error calculation based on both SS and DHS methods for abnormal earnings and Panel B reports the percentage price errors for stock prices. The table also provides percentage (percentage point) differences of each dollar (percentage pricing) error measurement based on the SS method with respect to the benchmark DHS method. 
Table 3.5: 1950-2017 In-sample Pricing and Forecast Errors: Non-FIPU Firms

\begin{tabular}{|c|c|c|c|c|c|c|c|c|c|c|c|c|}
\hline \multirow[b]{2}{*}{ Method } & \multirow[b]{2}{*}{ Variable } & \multirow[b]{2}{*}{ Index } & \multicolumn{6}{|c|}{$\begin{array}{c}\text { 1950-2017 Full Sample } \\
\text { Non-FIPU Firms }\end{array}$} & \multirow[b]{2}{*}{ MSE } & \multirow[b]{2}{*}{$\%$ diff } & \multirow[b]{2}{*}{ RMSE } & \multirow[b]{2}{*}{$\%$ diff } \\
\hline & & & MDE & $\%$ diff & STD & $\%$ diff & MADE & $\%$ diff & & & & \\
\hline \multicolumn{13}{|c|}{ Panel A: Abnormal Earnings } \\
\hline \multirow{3}{*}{ SS Method } & & DJIA & 0.0786 & $-180.82 \%$ & 0.2590 & $-14.22 \%$ & 0.1695 & $-21.57 \%$ & 0.0723 & $-26.62 \%$ & 0.2688 & $-14.34 \%$ \\
\hline & $x_{t}^{a}$ & & & & & & & & & & & \\
\hline & & S\&P 500 & 0.0869 & $-138.23 \%$ & 0.2262 & $-40.15 \%$ & 0.1373 & $-44.96 \%$ & 0.0579 & $-69.68 \%$ & 0.2407 & $-44.94 \%$ \\
\hline \multirow{2}{*}{ DHS Method } & & DJIA & -0.0973 & - & 0.3020 & - & 0.2161 & - & 0.0985 & - & 0.3138 & - \\
\hline & & S\&P 500 & -0.2273 & - & 0.3779 & - & 0.2496 & - & 0.1911 & - & 0.4371 & - \\
\hline Method & Variable & Index & MPE & $\%$ pt diff & STD & $\%$ pt diff & MAPE & $\%$ pt diff & MSPE & $\%$ pt diff & RMSPE & $\%$ pt diff \\
\hline \multicolumn{13}{|c|}{ Panel B: Stock Price } \\
\hline \multirow{3}{*}{ SS Method } & & DJIA & $51.05 \%$ & $-1.48 \%$ & $16.33 \%$ & $2.73 \%$ & $51.05 \%$ & $-1.48 \%$ & $28.69 \%$ & $-0.71 \%$ & $53.56 \%$ & $-0.66 \%$ \\
\hline & $P_{t}$ & & & & & & & & & & & \\
\hline & & S\&P 500 & $47.67 \%$ & $-3.00 \%$ & $20.72 \%$ & $4.58 \%$ & $48.78 \%$ & $-1.88 \%$ & $26.95 \%$ & $-1.27 \%$ & $51.91 \%$ & $-1.21 \%$ \\
\hline \multirow{3}{*}{ DHS Method } & & DJIA & $52.53 \%$ & - & $13.60 \%$ & - & $52.53 \%$ & - & $29.40 \%$ & - & $54.22 \%$ & - \\
\hline & $P_{t}$ & & & & & & & & & & & \\
\hline & & S\&P 500 & $50.67 \%$ & - & $16.14 \%$ & - & $50.67 \%$ & - & $28.22 \%$ & - & $53.12 \%$ & - \\
\hline
\end{tabular}

In-sample pricing and forecast errors table provides five error measurements for firms excluding financial institutions, insurance companies, and public utilities (non-FIPU firms) for the 1950-2017 full-sample period: Mean Dollar Error (MDE) or Mean Percentage Pricing Error (MPE), Standard Deviation (STD), Mean Absolute Dollar Error (MADE) or Mean Absolute Percentage Error (MAPE), Mean Squared Error (MSE) or Mean Squared Percentage Error (MSPE), and Root Mean Squared Error (RMSE) or Root Mean Squared Percentage Error (RMSPE). Panel A reports each dollar error calculation based on both SS and DHS methods for abnormal earnings and Panel B reports the percentage price errors for stock prices. The table also provides percentage (percentage point) differences of each dollar (percentage pricing) error measurement based on the SS method with respect to the benchmark DHS method. 
Table 3.6: 1976-2017 In-sample Pricing and Forecast Errors: FIPU Firms

\begin{tabular}{|c|c|c|c|c|c|c|c|c|c|c|c|c|}
\hline \multirow[b]{3}{*}{ Method } & \multirow[b]{3}{*}{ Variable } & \multirow[b]{3}{*}{ Index } & \multirow{2}{*}{\multicolumn{4}{|c|}{$\begin{array}{c}\text { 1976-2017 Sub-sample } \\
\text { FIPU Firms }\end{array}$}} & \multirow[b]{3}{*}{ MADE } & \multirow[b]{3}{*}{$\%$ diff } & \multirow[b]{3}{*}{ MSE } & \multirow[b]{3}{*}{$\%$ diff } & \multirow[b]{3}{*}{ RMSE } & \multirow[b]{3}{*}{$\%$ diff } \\
\hline & & & & & & & & & & & & \\
\hline & & & MDE & $\%$ diff & STD & $\%$ diff & & & & & & \\
\hline \multicolumn{13}{|c|}{ Panel A: Abnormal Earnings } \\
\hline & & DJIA & 0.1234 & $-158.83 \%$ & 1.3812 & $-3.76 \%$ & 0.8473 & $4.00 \%$ & 1.8776 & $-8.63 \%$ & 1.3703 & $-4.41 \%$ \\
\hline \multirow[t]{3}{*}{ SS Method } & $x_{t}^{a}$ & & & & & & & & & & & \\
\hline & & S\&P 500 & -0.0257 & $-96.48 \%$ & 3.4883 & $21.74 \%$ & 1.0465 & $34.44 \%$ & 11.8791 & $38.96 \%$ & 3.4466 & $17.88 \%$ \\
\hline & & DJIA & -0.2098 & - & 1.4352 & - & 0.8147 & - & 2.0549 & - & 1.4335 & - \\
\hline \multirow[t]{2}{*}{ DHS Method } & $x_{t}^{a}$ & & & & & & & & & & & \\
\hline & & S\&P 500 & -0.7307 & - & 2.8653 & - & 0.7784 & - & 8.5484 & - & 2.9238 & - \\
\hline Method & Variable & Index & MPE & $\%$ pt diff & STD & $\%$ pt diff & MAPE & $\%$ pt diff & MSPE & $\%$ pt diff & RMSPE & $\%$ pt diff \\
\hline \multicolumn{13}{|c|}{ Panel B: Stock Price } \\
\hline \multirow{3}{*}{ SS Method } & & DJIA & $31.51 \%$ & $7.04 \%$ & $32.54 \%$ & $-3.37 \%$ & $40.10 \%$ & $2.69 \%$ & $20.27 \%$ & $1.69 \%$ & $45.02 \%$ & $1.92 \%$ \\
\hline & $P_{t}$ & & & & & & & & & & & \\
\hline & & S\&P 500 & $35.40 \%$ & $17.64 \%$ & $65.34 \%$ & $31.11 \%$ & $45.77 \%$ & $12.02 \%$ & $54.21 \%$ & $39.62 \%$ & $73.63 \%$ & $35.42 \%$ \\
\hline \multirow{3}{*}{ DHS Method } & & DJIA & $24.47 \%$ & - & $35.91 \%$ & - & $37.41 \%$ & - & $18.57 \%$ & - & $43.10 \%$ & - \\
\hline & $P_{t}$ & & & & & & & & & & & \\
\hline & & S\&P 500 & $17.76 \%$ & - & $34.23 \%$ & - & $33.75 \%$ & - & $14.60 \%$ & - & $38.20 \%$ & - \\
\hline
\end{tabular}

In-sample pricing and forecast errors table provides five error measurements for firms for financial institutions, insurance companies, and public utilities (non-FIPU firms) for the 1976-2017 sub-sample period: Mean Dollar Error (MDE) or Mean Percentage Pricing Error (MPE), Standard Deviation (STD), Mean Absolute Dollar Error (MADE) or Mean Absolute Percentage Error (MAPE), Mean Squared Error (MSE) or Mean Squared Percentage Error (MSPE), and Root Mean Squared Error (RMSE) or Root Mean Squared Percentage Error (RMSPE). Panel A reports each dollar error calculation based on both SS and DHS methods for abnormal earnings and Panel B reports the percentage price errors for stock prices. The table also provides percentage (percentage point) differences of each dollar (percentage pricing) error measurement based on the SS method with respect to the benchmark DHS method. 
Table 3.7: 1950-2017 In-sample Pricing and Forecast Errors: FIPU Firms

\begin{tabular}{|c|c|c|c|c|c|c|c|c|c|c|c|c|}
\hline \multicolumn{13}{|c|}{ 1950-2017 Full Sample } \\
\hline \multirow[b]{2}{*}{ Method } & \multirow[b]{2}{*}{ Variable } & \multirow[b]{2}{*}{ Index } & \multirow[b]{2}{*}{ MDE } & \multicolumn{3}{|c|}{ FIPU Firms } & \multirow[b]{2}{*}{ MADE } & \multirow[b]{2}{*}{$\%$ diff } & \multirow[b]{2}{*}{ MSE } & \multirow[b]{2}{*}{$\%$ diff } & \multirow[b]{2}{*}{ RMSE } & \multirow[b]{2}{*}{$\%$ diff } \\
\hline & & & & $\%$ diff & STD & $\%$ diff & & & & & & \\
\hline \multicolumn{13}{|c|}{ Panel A: Abnormal Earnings } \\
\hline \multirow{3}{*}{ SS Method } & & DJIA & 0.1076 & $-151.32 \%$ & 1.3050 & $-9.07 \%$ & 0.7646 & $-6.15 \%$ & 1.6785 & $-18.32 \%$ & 1.2956 & $-9.62 \%$ \\
\hline & $x_{t}^{a}$ & & & & & & & & & & & \\
\hline & & S\&P 500 & -0.0205 & $-97.19 \%$ & 3.0681 & $7.08 \%$ & 0.8195 & $5.27 \%$ & 9.2395 & $8.08 \%$ & 3.0397 & $3.96 \%$ \\
\hline \multirow{3}{*}{ DHS Method } & & DJIA & -0.2098 & - & 1.4352 & - & 0.8147 & - & 2.0549 & - & 1.4335 & - \\
\hline & $x_{t}^{a}$ & & & & & & & & & & & \\
\hline & & S\&P 500 & -0.7307 & - & 2.8653 & - & 0.7784 & - & 8.5484 & - & 2.9238 & - \\
\hline Method & Variable & Index & MPE & $\%$ pt diff & STD & $\%$ pt diff & MAPE & $\%$ pt diff & MSPE & $\%$ pt diff & RMSPE & $\%$ pt diff \\
\hline \multicolumn{13}{|c|}{ Panel B: Stock Price } \\
\hline \multirow{3}{*}{ SS Method } & & DJIA & $27.66 \%$ & $3.19 \%$ & $33.02 \%$ & $-2.89 \%$ & $37.29 \%$ & $-0.12 \%$ & $18.32 \%$ & $-0.25 \%$ & $42.81 \%$ & $-0.29 \%$ \\
\hline & $P_{t}$ & & & & & & & & & & & \\
\hline & & S\&P 500 & $32.53 \%$ & $14.76 \%$ & $59.89 \%$ & $25.66 \%$ & $43.95 \%$ & $10.20 \%$ & $45.78 \%$ & $31.19 \%$ & $67.66 \%$ & $29.46 \%$ \\
\hline \multirow{3}{*}{ DHS Method } & & DJIA & $24.47 \%$ & - & $35.91 \%$ & - & $37.41 \%$ & - & $18.57 \%$ & - & $43.10 \%$ & - \\
\hline & $P_{t}$ & & & & & & & & & & & \\
\hline & & S\&P 500 & $17.76 \%$ & - & $34.23 \%$ & - & $33.75 \%$ & - & $14.60 \%$ & - & $38.20 \%$ & - \\
\hline
\end{tabular}

In-sample pricing and forecast errors table provides five mean error measurements for financial institutions, insurance companies, and public utilities (FIPU firms) for the 1950-2017 full-sample period: mean error (ME), mean absolute error (MAE), mean squared error (MSE), and root mean squared error (RMSE). Panel A reports each mean error calculation based on both SS and DHS methods for abnormal earnings and Panel $B$ reports the same for stock prices. The table also provides percentage differences of each mean error measurement based on the SS method with respect to the benchmark DHS method. 
Table 3.8: Quantitative Assessment of Model Fit: SS Method and DSH Method

$$
P_{t}=\beta_{0}+\beta_{1} \hat{P}_{t}+\tilde{\eta}_{t},
$$

\begin{tabular}{|c|c|c|c|c|c|}
\hline Method & Index & $\beta_{0}$ & $\beta_{1}$ & t-stat & Adj. $R^{2}$ \\
\hline \multicolumn{6}{|c|}{ Panel A: Non-FIPU Firms } \\
\hline \multirow{4}{*}{ SS Method } & DJIA & 6.46 & 1.92 & 6.49 & $72.09 \%$ \\
\hline & & $(2.36)$ & $(0.30)$ & & \\
\hline & S\&P 500 & 7.93 & 2.38 & 13.74 & $53.77 \%$ \\
\hline & & $(1.40)$ & $(0.17)$ & & \\
\hline \multirow{4}{*}{ DHS Method } & DJIA & 7.01 & 1.97 & 8.18 & $72.23 \%$ \\
\hline & & $(2.54)$ & $(0.24)$ & & \\
\hline & S\&P 500 & 6.74 & 2.31 & 13.53 & $59.90 \%$ \\
\hline & & $(1.69)$ & $(0.17)$ & & \\
\hline \multicolumn{6}{|c|}{ Panel B: FIPU Firms } \\
\hline \multirow{4}{*}{ SS Method } & DJIA & 14.07 & 1.20 & 9.67 & $71.67 \%$ \\
\hline & & $(3.95)$ & $(0.12)$ & & \\
\hline & S\&P 500 & 38.12 & 0.30 & 1.17 & $12.68 \%$ \\
\hline & & $(6.33)$ & $(0.26)$ & & \\
\hline \multirow{4}{*}{ DHS Method } & DJIA & 13.22 & 1.24 & 9.75 & $73.06 \%$ \\
\hline & & $(4.11)$ & $(0.13)$ & & \\
\hline & S\&P 500 & -5.03 & 1.92 & 11.85 & $78.84 \%$ \\
\hline & & $(3.23)$ & $(0.16)$ & & \\
\hline
\end{tabular}

Simple regression analysis provide additional evidence of the in-sample pricing performance relative to market prices under both SS and DHS estimation methods using the full sample. $\beta$ coefficients, $\mathrm{t}$-statistic for the null hypothesis of $B_{1}=0$, and the adjusted $R^{2}$ are reported in Panel A for firms excluding financial institutions, insurance companies, and public utilities (non-FIPU firms). The same are reported in Panel B for financial institutions, insurance companies, and public utilities (FIPU firms). The SS Method represents the in-sample implied prices using the state space application via the Kalman filtering recursive procedure. The DHS Method represents the insample implied prices based on two unconditional pooled regressions of Eqs. (7a) and (7b). Numbers in parentheses for $\beta$ coefficients are their standard errors computed using the Newey-West estimator to control for heteroscedasticity. 
Table 3.9: 3-year Rolling-Window Out-of-Sample Performance Metrics

\begin{tabular}{|c|c|c|c|c|c|c|c|c|c|c|c|}
\hline & & & & 1976-20 & 17 Sub-san & ple & & & & & \\
\hline Method & Variable & MPE & $\%$ pt diff & STD & $\%$ pt diff & MAPE & $\%$ pt diff & MSPE & $\%$ pt diff & RMSPE & $\%$ pt diff \\
\hline \multicolumn{12}{|c|}{ Panel A: Stock Price for Non-FIPU Firms } \\
\hline SS Method & $P_{t}$ & $24.36 \%$ & $-32.55 \%$ & $47.12 \%$ & $32.84 \%$ & $41.98 \%$ & $-14.93 \%$ & $27.61 \%$ & $-6.77 \%$ & $52.54 \%$ & $-6.09 \%$ \\
\hline DHS Method & $P_{t}$ & $56.91 \%$ & - & $14.28 \%$ & - & $56.91 \%$ & - & $34.38 \%$ & - & $58.63 \%$ & - \\
\hline \multicolumn{12}{|c|}{ Panel B: Stock Price for FIPU Firms } \\
\hline SS Method & $P_{t}$ & $20.68 \%$ & $14.98 \%$ & $56.20 \%$ & $-6.25 \%$ & $46.37 \%$ & $4.03 \%$ & $35.11 \%$ & $-3.25 \%$ & $59.25 \%$ & $-2.68 \%$ \\
\hline DHS Method & $P_{t}$ & $5.69 \%$ & - & $62.46 \%$ & - & $42.33 \%$ & - & $38.36 \%$ & - & $61.93 \%$ & - \\
\hline
\end{tabular}

3-year rolling-window out-of-sample pricing table provides five error measurements for 24 firms during the 1976-2017 sub-sample period: Mean Percentage Pricing Error (MPE), Standard Deviation (STD), Mean Absolute Percentage Error (MAPE), Mean Squared Percentage Error (MSPE), and Root Mean Squared Percentage Error (RMSPE). Panel A reports each percentage pricing error calculation based on both SS and DHS methods for 14 non-FIPU firms and Panel B reports the percentage pricing errors for 10 FIPU firms. The table also provides percentage (percentage point) differences of each percentage pricing error measurement based on the SS method with respect to the benchmark DHS method. 
Table 3.10: 5-Year Rolling-Window Out-of-Sample Valuation Performance Metrics

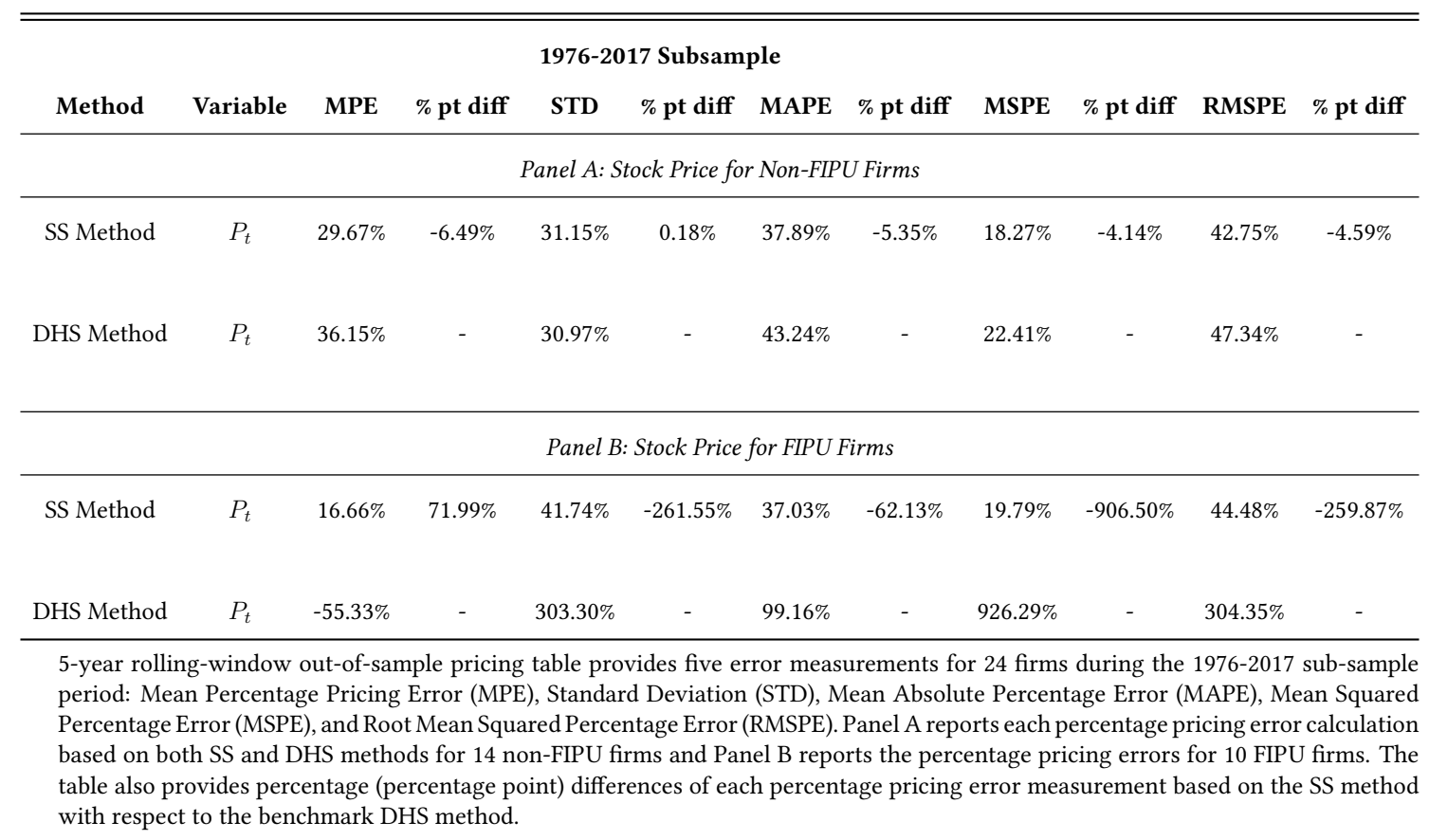


Table 3.11: Pricing-error Autocorrelations for 24 Stocks: 3-year Rolling Sub-sample

\begin{tabular}{|c|c|c|c|c|c|c|c|c|c|}
\hline \multicolumn{10}{|c|}{ Autocorrelations } \\
\hline No. & Stock & Lag 1 & Lag 2 & Lag 3 & Lag 5 & $Q(3)$ & $\mathrm{p}$-value & $Q(5)$ & p-value \\
\hline \multicolumn{10}{|c|}{ Panel A: Non-FIPU Firms } \\
\hline 1 & Apple & 0.29 & 0.04 & -0.09 & 0.02 & 3.48 & 0.323 & 3.60 & 0.608 \\
\hline 2 & Verizon & 0.42 & -0.14 & -0.13 & -0.01 & 7.09 & 0.069 & 7.16 & 0.209 \\
\hline 3 & Chevron & 0.13 & 0.05 & 0.11 & -0.03 & 2.12 & 0.548 & 2.31 & 0.804 \\
\hline 4 & Dow DuPont & 0.10 & -0.04 & -0.15 & -0.10 & 2.03 & 0.567 & 3.76 & 0.585 \\
\hline 5 & ExxonMobil & 0.45 & 0.06 & 0.04 & 0.17 & 13.93 & 0.003 & 17.99 & 0.003 \\
\hline 6 & Home Depot & 0.39 & 0.07 & 0.11 & 0.13 & 6.15 & 0.104 & 7.87 & 0.164 \\
\hline 7 & Intel & -0.04 & -0.06 & 0.12 & -0.11 & 0.98 & 0.806 & 1.64 & 0.896 \\
\hline 8 & IBM & 0.29 & -0.01 & -0.06 & -0.03 & 5.93 & 0.115 & 12.19 & 0.032 \\
\hline 9 & Merck \& Co & 0.09 & -0.04 & -0.07 & -0.04 & 0.99 & 0.804 & 1.87 & 0.867 \\
\hline 10 & Nike & 0.24 & -0.12 & 0.04 & -0.15 & 2.74 & 0.433 & 3.74 & 0.587 \\
\hline 11 & United Tech. & -0.04 & -0.05 & -0.01 & -0.22 & 0.24 & 0.971 & 4.04 & 0.544 \\
\hline 12 & Walmart & 0.26 & 0.09 & 0.06 & 0.01 & 3.72 & 0.293 & 4.05 & 0.543 \\
\hline 13 & Microsoft & 0.61 & 0.11 & 0.02 & -0.08 & 12.09 & 0.007 & 12.55 & 0.028 \\
\hline 14 & Cisco Systems & 0.16 & 0.01 & 0.31 & -0.03 & 3.50 & 0.320 & 3.73 & 0.588 \\
\hline \multicolumn{10}{|c|}{ Panel B: FIPU Firms } \\
\hline 1 & Pinnacle & 0.19 & 0.04 & 0.07 & 0.14 & 2.31 & 0.510 & 4.31 & 0.506 \\
\hline 2 & American Express & 0.06 & -0.10 & 0.04 & -0.10 & 0.72 & 0.868 & 1.64 & 0.897 \\
\hline 3 & JPM Chase & 0.53 & 0.38 & 0.49 & 0.31 & 32.92 & 0.000 & 47.67 & 0.000 \\
\hline 4 & Aon & 0.33 & 0.19 & 0.23 & 0.42 & 9.12 & 0.028 & 22.38 & 0.000 \\
\hline 5 & Duke Energy & 0.45 & 0.28 & 0.28 & 0.28 & 19.94 & 0.000 & 28.70 & 0.000 \\
\hline 6 & BofA & -0.05 & -0.11 & 0.06 & -0.01 & 0.85 & 0.836 & 0.94 & 0.967 \\
\hline 7 & Nisource & 0.23 & -0.07 & -0.07 & -0.02 & 3.58 & 0.310 & 3.62 & 0.606 \\
\hline 8 & UnitedHealth & 0.40 & 0.15 & -0.19 & -0.03 & 7.24 & 0.065 & 7.51 & 0.185 \\
\hline 9 & Goldman Sachs & 0.41 & 0.00 & 0.04 & -0.29 & 3.15 & 0.369 & 8.06 & 0.153 \\
\hline 10 & Visa & -0.15 & -0.13 & -0.34 & -1.32 & 2.23 & 0.527 & 87.65 & 0.000 \\
\hline
\end{tabular}

We report the autocorrelations of out-of-sample percentage pricing errors based on a 3year rolling-window for the SS estimation approach. We also report the Ljung-Box Qstatistic at 3- and 5-year lags and the corresponding p-values for the $\chi^{2}$ test. The test is based on the null hypothesis of the absence of autocorrelation in the pricing errors. $\mathrm{Q}(3)$ and $Q(5)$ are each $\chi^{2}$-distributed with 3 and 5 degrees of freedom, respectively. 
Table 3.12: Pricing-error Autocorrelations for 24 Stocks: 5-year Rolling Sub-sample

\begin{tabular}{|c|c|c|c|c|c|c|c|c|c|}
\hline \multicolumn{10}{|c|}{ Autocorrelations } \\
\hline No. & Stock & Lag 1 & Lag 2 & Lag 3 & Lag 5 & $Q(3)$ & $\mathrm{p}$-value & $Q(5)$ & p-value \\
\hline \multicolumn{10}{|c|}{ Panel A: Non-FIPU Firms } \\
\hline 1 & Apple & 0.36 & 0.16 & 0.07 & -0.13 & 5.61 & 0.132 & 6.82 & 0.234 \\
\hline 2 & Verizon & 0.41 & 0.09 & 0.03 & 0.05 & 5.57 & 0.135 & 6.69 & 0.245 \\
\hline 3 & Chevron & 0.64 & 0.32 & 0.29 & 0.24 & 38.77 & 0.000 & 47.30 & 0.000 \\
\hline 4 & Dow DuPont & 0.24 & 0.14 & 0.00 & 0.06 & 4.11 & 0.250 & 4.49 & 0.481 \\
\hline 5 & ExxonMobil & 0.60 & 0.35 & 0.27 & 0.23 & 36.21 & 0.000 & 43.03 & 0.000 \\
\hline 6 & Home Depot & 0.71 & 0.44 & 0.06 & -0.02 & 24.16 & 0.000 & 24.32 & 0.000 \\
\hline 7 & Intel & 0.03 & -0.07 & 0.00 & -0.07 & 0.24 & 0.971 & 0.48 & 0.993 \\
\hline 8 & IBM & 0.44 & 0.02 & 0.06 & 0.02 & 12.87 & 0.005 & 13.13 & 0.022 \\
\hline 9 & Merck \& Co & 0.38 & 0.01 & -0.12 & -0.16 & 10.28 & 0.016 & 13.40 & 0.020 \\
\hline 10 & Nike & 0.68 & 0.37 & 0.13 & -0.22 & 21.93 & 0.000 & 25.50 & 0.000 \\
\hline 11 & United Tech. & 0.01 & -0.03 & -0.03 & -0.13 & 0.12 & 0.989 & 1.37 & 0.928 \\
\hline 12 & Walmart & 0.63 & 0.25 & 0.19 & 0.55 & 21.39 & 0.000 & 45.58 & 0.000 \\
\hline 13 & Microsoft & 0.15 & -0.02 & -0.05 & -0.15 & 0.75 & 0.861 & 2.50 & 0.777 \\
\hline 14 & Cisco Systems & 0.27 & 0.09 & 0.17 & -0.01 & 2.93 & 0.403 & 3.06 & 0.691 \\
\hline \multicolumn{10}{|c|}{ Panel B: FIPU Firms } \\
\hline 1 & Pinnacle & 0.37 & 0.10 & 0.21 & 0.33 & 10.41 & 0.015 & 18.54 & 0.002 \\
\hline 2 & American Express & 0.61 & 0.37 & 0.24 & -0.02 & 24.33 & 0.000 & 24.75 & 0.000 \\
\hline 3 & JPM Chase & 0.54 & 0.44 & 0.48 & 0.33 & 33.72 & 0.000 & 46.03 & 0.000 \\
\hline 4 & Aon & 0.70 & 0.42 & 0.27 & 0.22 & 32.01 & 0.000 & 36.69 & 0.000 \\
\hline 5 & Duke Energy & 0.04 & -0.03 & 0.04 & 0.01 & 0.20 & 0.978 & 0.23 & 0.999 \\
\hline 6 & BofA & 0.28 & 0.01 & 0.38 & 0.07 & 10.78 & 0.013 & 16.16 & 0.006 \\
\hline 7 & Nisource & 0.29 & 0.14 & 0.06 & 0.33 & 5.76 & 0.124 & 18.58 & 0.002 \\
\hline 8 & UnitedHealth & 0.54 & 0.11 & 0.04 & -0.08 & 9.62 & 0.022 & 9.86 & 0.079 \\
\hline 9 & Goldman Sachs & 0.38 & -0.34 & -0.12 & -0.10 & 4.71 & 0.194 & 5.03 & 0.412 \\
\hline 10 & Visa & -0.85 & 0.48 & -0.40 & NA & 12.29 & 0.006 & NA & NA \\
\hline
\end{tabular}

We report the autocorrelations of out-of-sample percentage pricing errors based on a 5year rolling-window for the SS estimation approach. We also report the Ljung-Box Qstatistic at 3- and 5-year lags and the corresponding p-values for the $\chi^{2}$ test. The test is based on the null hypothesis of the absence of autocorrelation in the pricing errors. $\mathrm{Q}(3)$ and $Q(5)$ are each $\chi^{2}$-distributed with 3 and 5 degrees of freedom, respectively. 
Table 3.13: Cross-correlations in Mispricing of 24 stocks: 3year Rolling Sub-sample

\begin{tabular}{|c|c|c|c|c|c|}
\hline \multicolumn{6}{|c|}{ With IBM } \\
\hline No. & Stock & $b$ & se & t-stat & Adj. R2 \\
\hline \multicolumn{6}{|c|}{ Panel A: Non-FIPU Firms } \\
\hline 1 & Apple & -0.07 & 0.08 & -0.81 & $-2.89 \%$ \\
\hline 2 & Verizon & -0.02 & 0.05 & -0.47 & $-3.47 \%$ \\
\hline 3 & Chevron & 0.14 & 0.12 & 1.24 & $-0.79 \%$ \\
\hline 4 & Dow DuPont & 0.12 & 0.07 & 1.84 & $-1.24 \%$ \\
\hline 5 & ExxonMobil & 0.23 & 0.15 & 1.58 & $0.80 \%$ \\
\hline 6 & Home Depot & 0.04 & 0.03 & 1.51 & $-1.18 \%$ \\
\hline 7 & Intel & -0.16 & 0.11 & -1.40 & $1.81 \%$ \\
\hline 8 & IBM & NA & NA & NA & NA \\
\hline 9 & Merck \& Co & 0.08 & 0.12 & 0.64 & $-1.28 \%$ \\
\hline 10 & Nike & 0.04 & 0.05 & 0.84 & $-2.59 \%$ \\
\hline 11 & United Tech. & 0.21 & 0.12 & 1.71 & $-0.81 \%$ \\
\hline 12 & Walmart & 0.02 & 0.02 & 1.20 & $-2.29 \%$ \\
\hline 13 & Microsoft & 0.00 & 0.04 & -0.03 & $-3.85 \%$ \\
\hline 14 & Cisco Systems & -0.48 & 0.17 & -2.78 & $12.46 \%$ \\
\hline \multicolumn{6}{|c|}{ With UnitedHealth } \\
\hline No. & Stock & $b$ & se & t-stat & Adj. R2 \\
\hline \multicolumn{6}{|c|}{ Panel B: FIPU Firms } \\
\hline 1 & Pinnacle & -0.20 & 0.12 & -1.61 & $-0.90 \%$ \\
\hline 2 & American Express & 0.05 & 0.08 & 0.71 & $-2.50 \%$ \\
\hline 3 & JPM Chase & -0.06 & 0.08 & -0.74 & $-0.72 \%$ \\
\hline 4 & Aon & -0.03 & 0.03 & -0.89 & $-2.67 \%$ \\
\hline 5 & Duke Energy & -0.12 & 0.14 & -0.89 & $3.33 \%$ \\
\hline 6 & BofA & 0.00 & 0.10 & -0.02 & $-3.57 \%$ \\
\hline 7 & Nisource & -0.15 & 0.15 & -1.03 & $1.99 \%$ \\
\hline 8 & UnitedHealth & NA & NA & NA & NA \\
\hline 9 & Goldman Sachs & -0.31 & 0.50 & -0.63 & $-6.10 \%$ \\
\hline 10 & Visa & 0.69 & 0.24 & 2.89 & $58.67 \%$ \\
\hline
\end{tabular}

We examine the link between model pricing errors of stocks under the SS estimation method with a simple time-series regression using the 3-year rolling subsample. We use the pricing error for IBM and UnitedHealth (both arbitrarily chosen) as regressors for each non-FIPU and FIPU stock, respectively. We report the slope coefficient $b$ and its standard error using the Newey-West estimator, the t-statistic under the null hypothesis of $b=0$, and the adjusted $R^{2}$ for non-FIPU and FIPU stocks in Panels A and B, respectively. 
Table 3.14: Cross-correlations in Mispricing of 24 stocks: 5year Rolling Sub-sample

\begin{tabular}{|c|c|c|c|c|c|}
\hline \multicolumn{6}{|c|}{ With IBM } \\
\hline No. & Stock & $b$ & se & t-stat & Adj. R2 \\
\hline \multicolumn{6}{|c|}{ Panel A: Non-FIPU Firms } \\
\hline 1 & Apple & -0.07 & 0.08 & -0.81 & $-2.89 \%$ \\
\hline 2 & Verizon & -0.02 & 0.05 & -0.47 & $-3.47 \%$ \\
\hline 3 & Chevron & 0.14 & 0.12 & 1.24 & $-0.79 \%$ \\
\hline 4 & Dow DuPont & 0.12 & 0.07 & 1.84 & $-1.24 \%$ \\
\hline 5 & ExxonMobil & 0.23 & 0.15 & 1.58 & $0.80 \%$ \\
\hline 6 & Home Depot & 0.04 & 0.03 & 1.51 & $-1.18 \%$ \\
\hline 7 & Intel & -0.16 & 0.11 & -1.40 & $1.81 \%$ \\
\hline 8 & IBM & NA & NA & NA & NA \\
\hline 9 & Merck \& Co & 0.08 & 0.12 & 0.64 & $-1.28 \%$ \\
\hline 10 & Nike & 0.04 & 0.05 & 0.84 & $-2.59 \%$ \\
\hline 11 & United Tech. & 0.21 & 0.12 & 1.71 & $-0.81 \%$ \\
\hline 12 & Walmart & 0.02 & 0.02 & 1.20 & $-2.29 \%$ \\
\hline 13 & Microsoft & 0.00 & 0.04 & -0.03 & $-3.85 \%$ \\
\hline 14 & Cisco Systems & -0.48 & 0.17 & -2.78 & $12.46 \%$ \\
\hline \multicolumn{6}{|c|}{ With UnitedHealth } \\
\hline No. & Stock & $b$ & se & t-stat & Adj. R2 \\
\hline \multicolumn{6}{|c|}{ Panel B: FIPU Firms } \\
\hline 1 & Pinnacle & -0.20 & 0.12 & -1.61 & $-0.90 \%$ \\
\hline 2 & American Express & 0.05 & 0.08 & 0.71 & $-2.50 \%$ \\
\hline 3 & JPM Chase & -0.06 & 0.08 & -0.74 & $-0.72 \%$ \\
\hline 4 & Aon & -0.03 & 0.03 & -0.89 & $-2.67 \%$ \\
\hline 5 & Duke Energy & -0.12 & 0.14 & -0.89 & $3.33 \%$ \\
\hline 6 & BofA & 0.00 & 0.10 & -0.02 & $-3.57 \%$ \\
\hline 7 & Nisource & -0.15 & 0.15 & -1.03 & $1.99 \%$ \\
\hline 8 & UnitedHealth & NA & NA & NA & NA \\
\hline 9 & Goldman Sachs & -0.31 & 0.50 & -0.63 & $-6.10 \%$ \\
\hline 10 & Visa & 0.69 & 0.24 & 2.89 & $58.67 \%$ \\
\hline
\end{tabular}

We examine the link between model pricing errors of stocks under the SS estimation method with a simple time-series regression using the 5-year rolling subsample. We use the pricing error for IBM and UnitedHealth (both arbitrarily chosen) as regressors for each non-FIPU and FIPU stock, respectively. We report the slope coefficient $b$ and its standard error using the Newey-West estimator, the t-statistic under the null hypothesis of $b=0$, and the adjusted $R^{2}$ for non-FIPU and FIPU stocks in Panels A and B, respectively. 


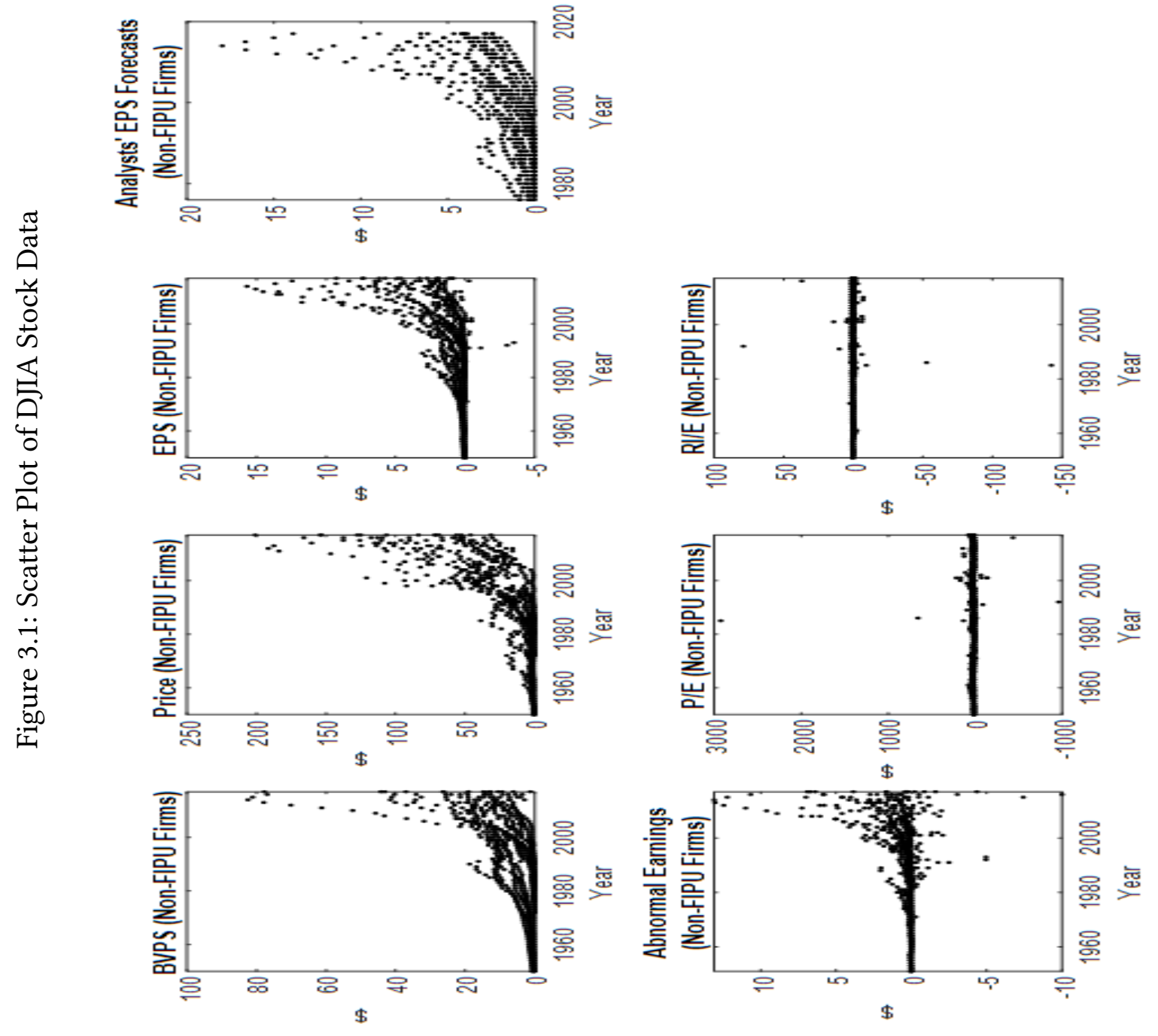




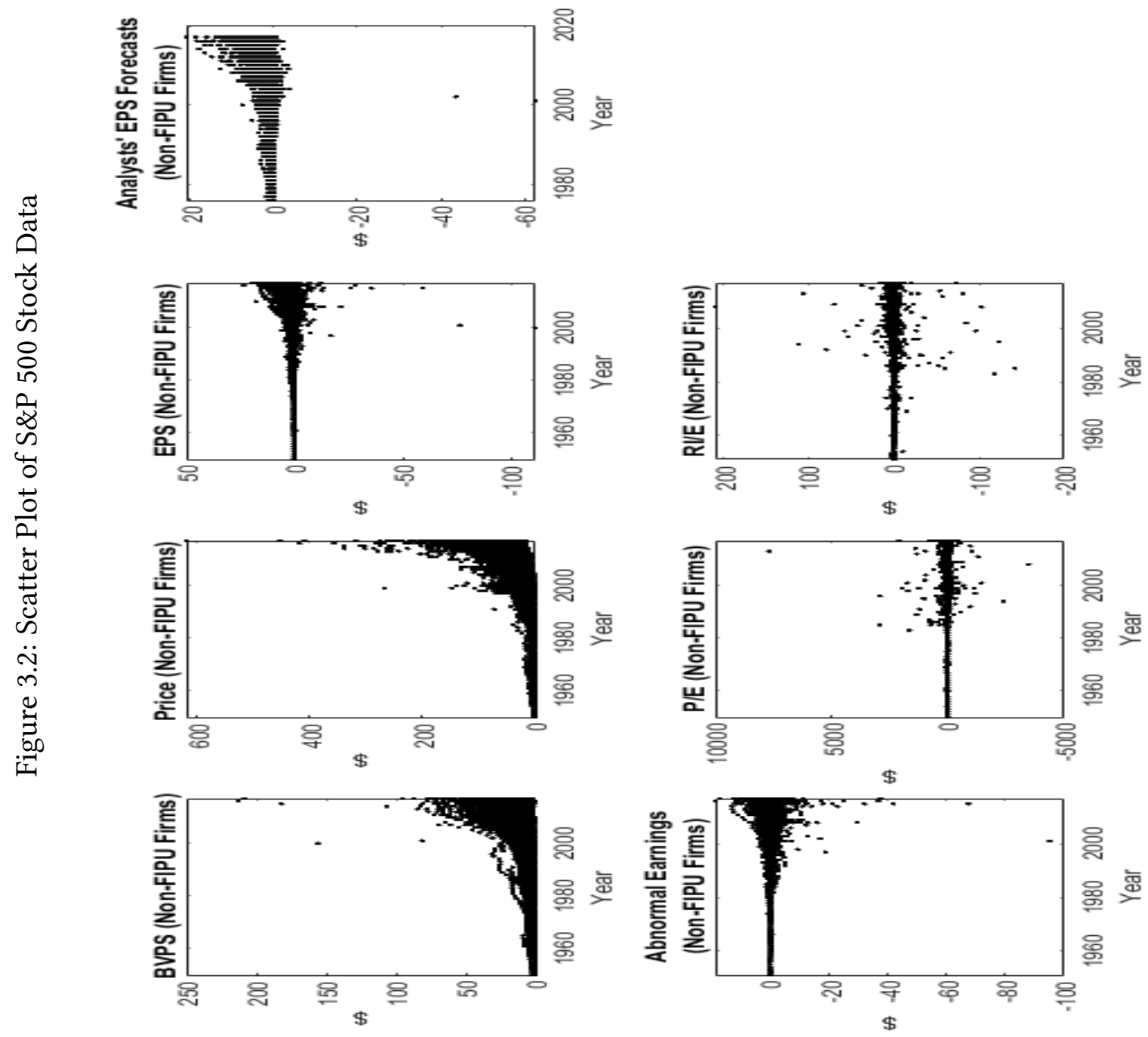




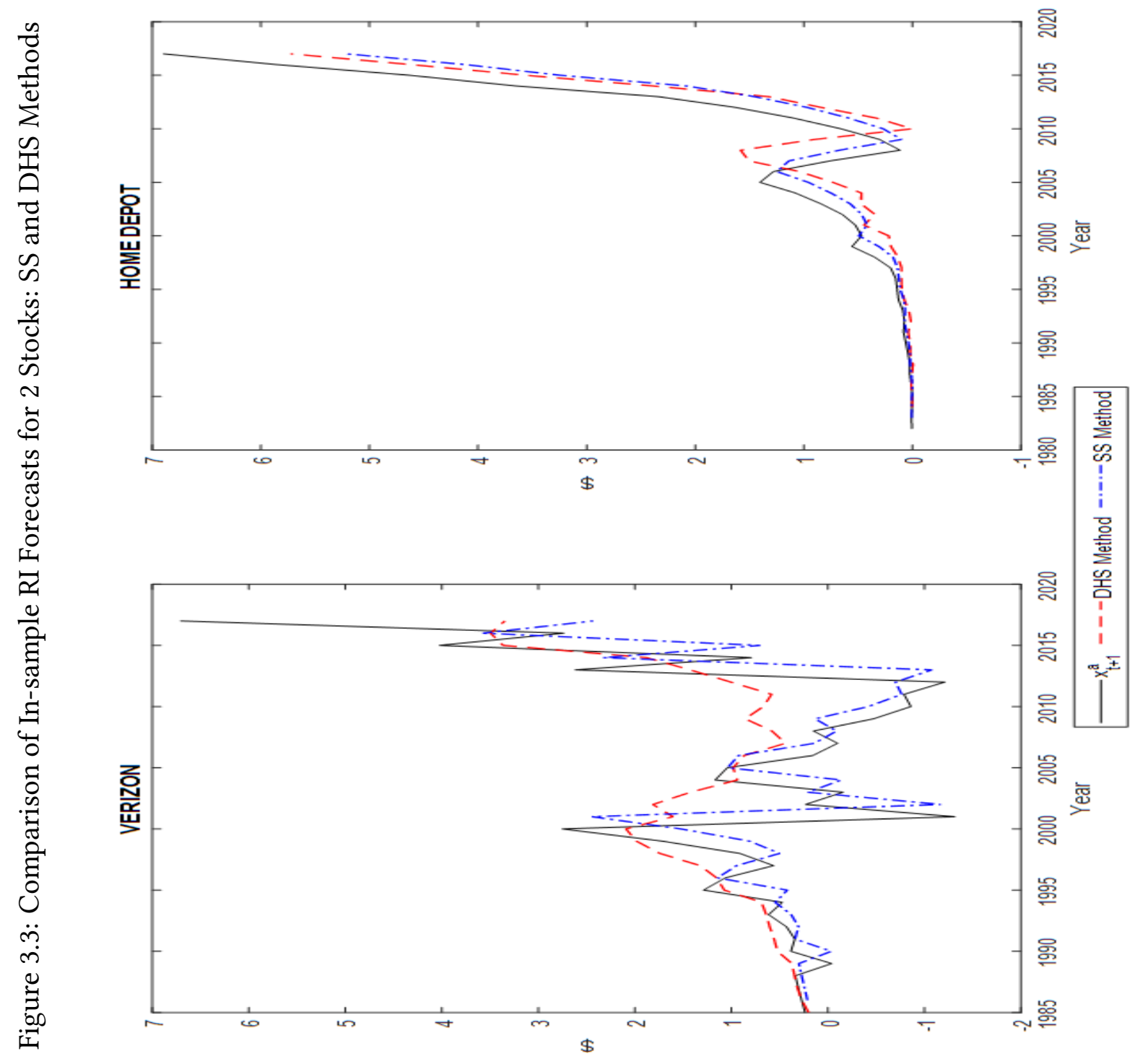




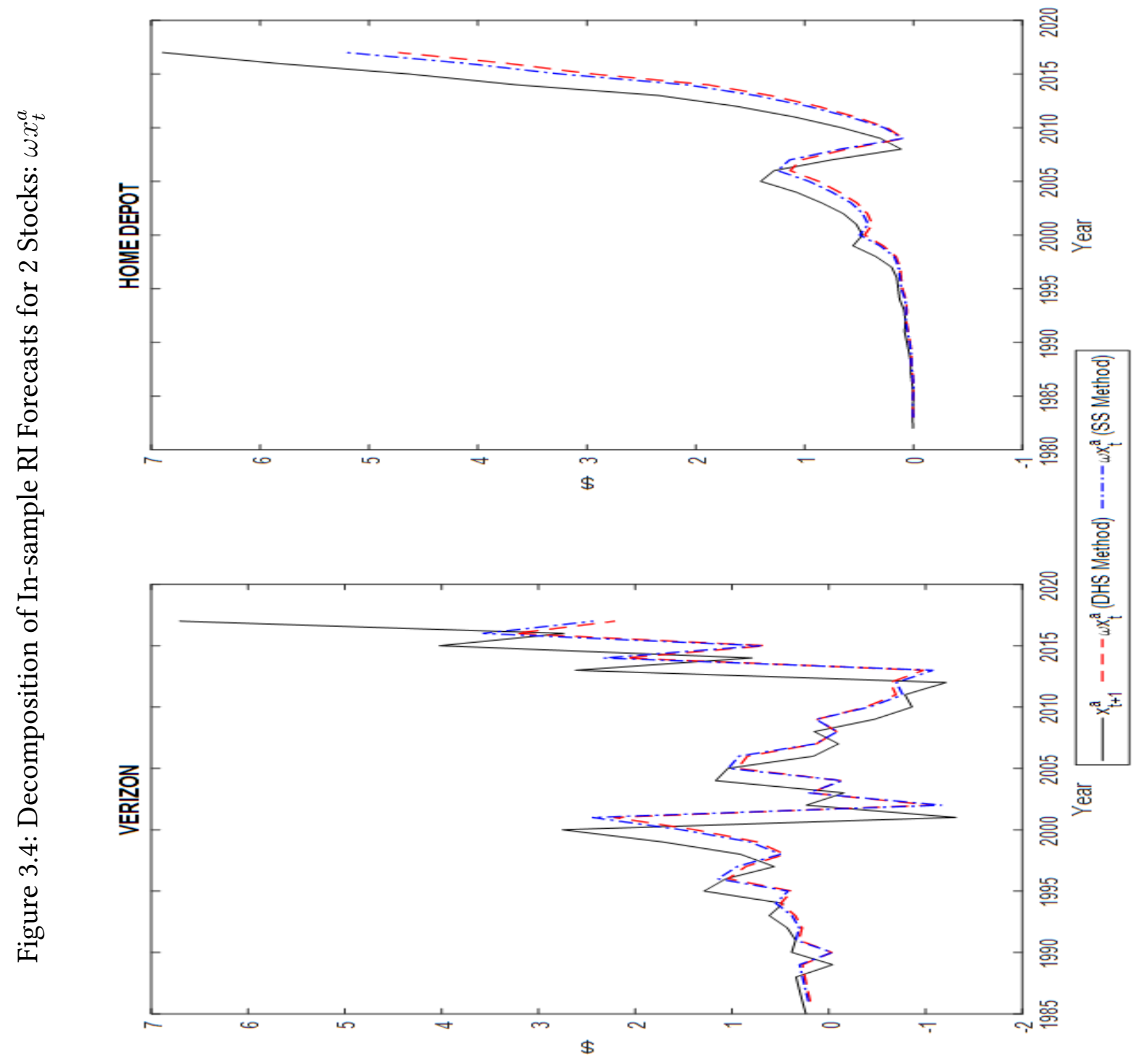




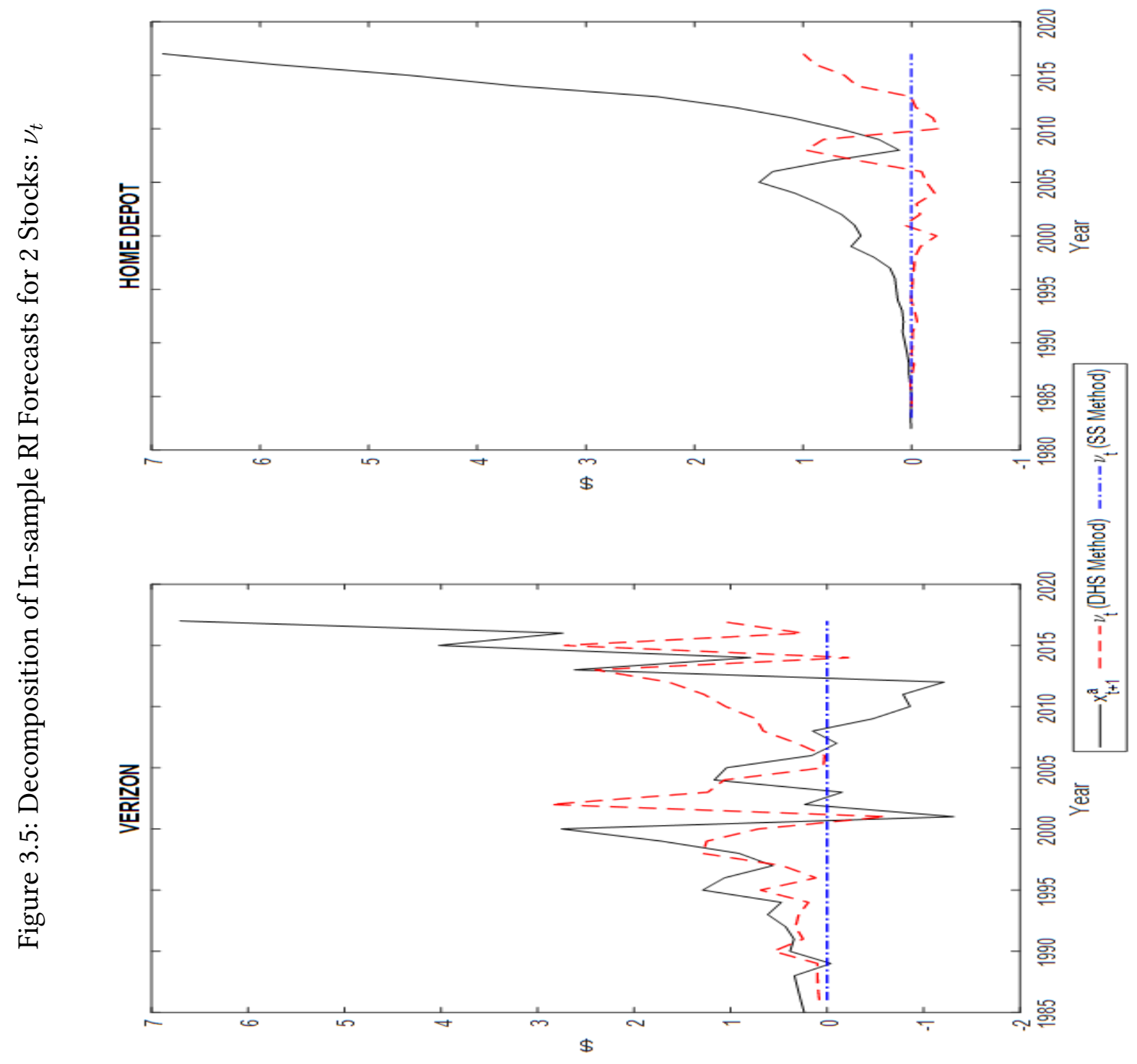




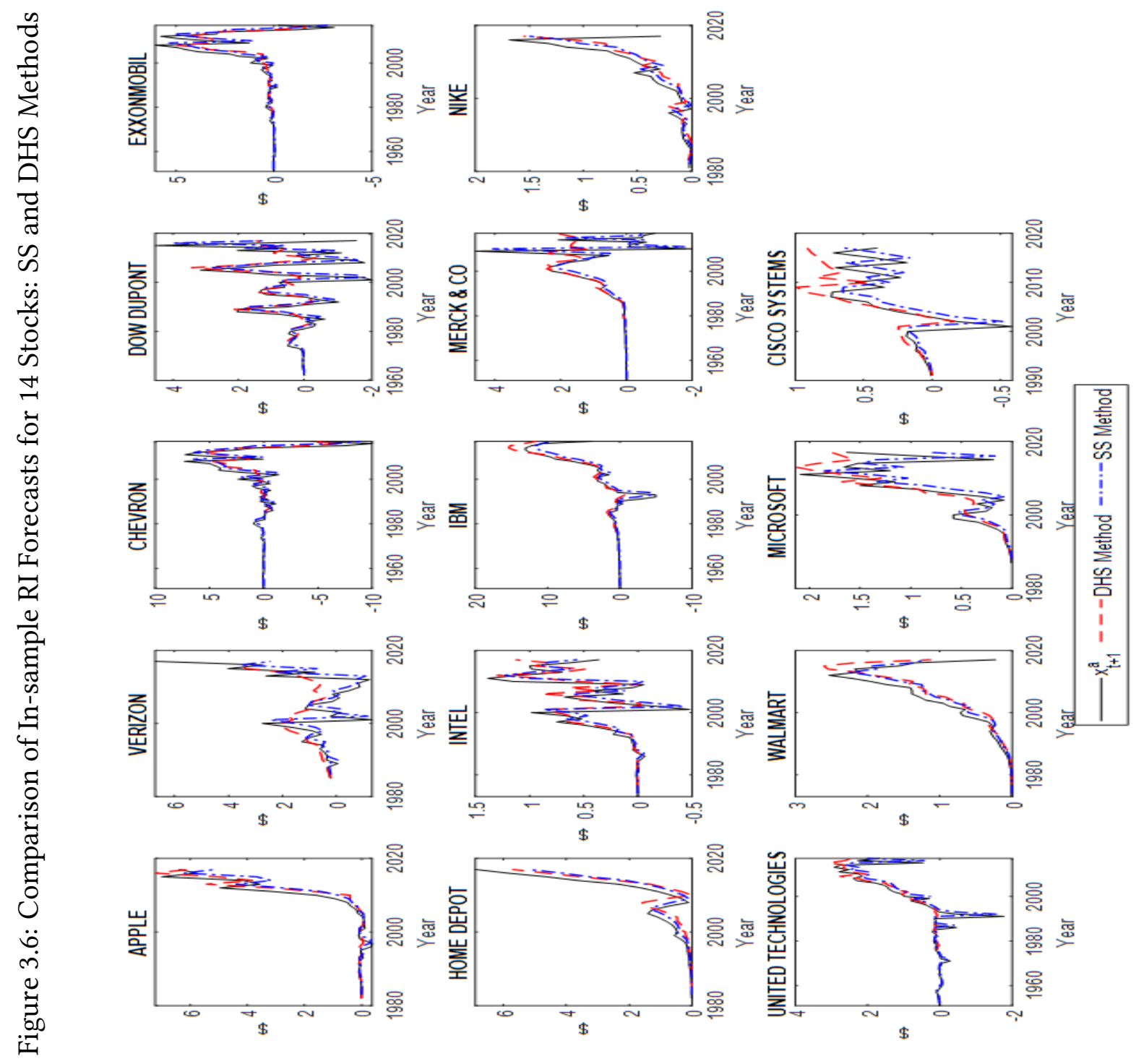




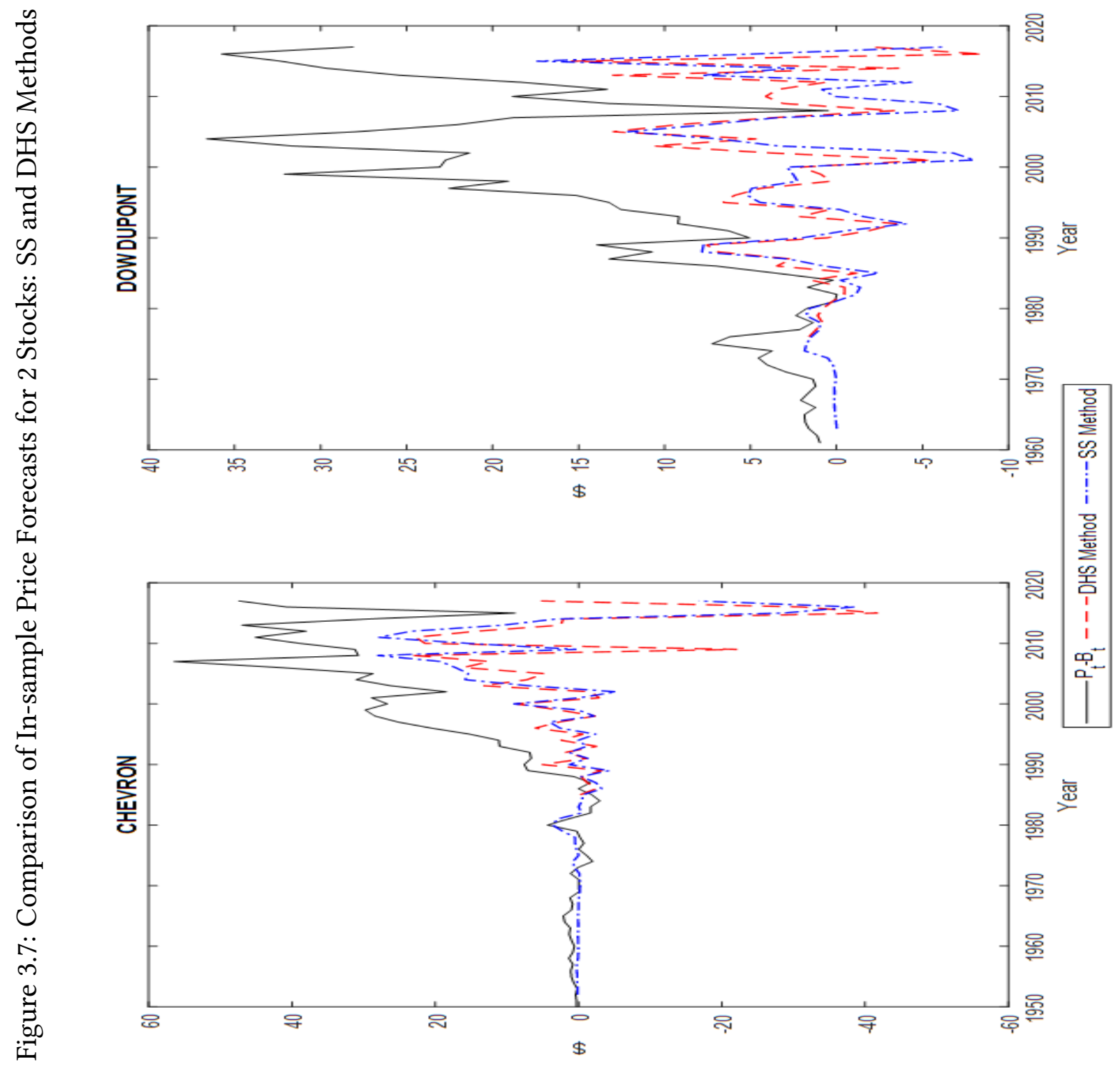




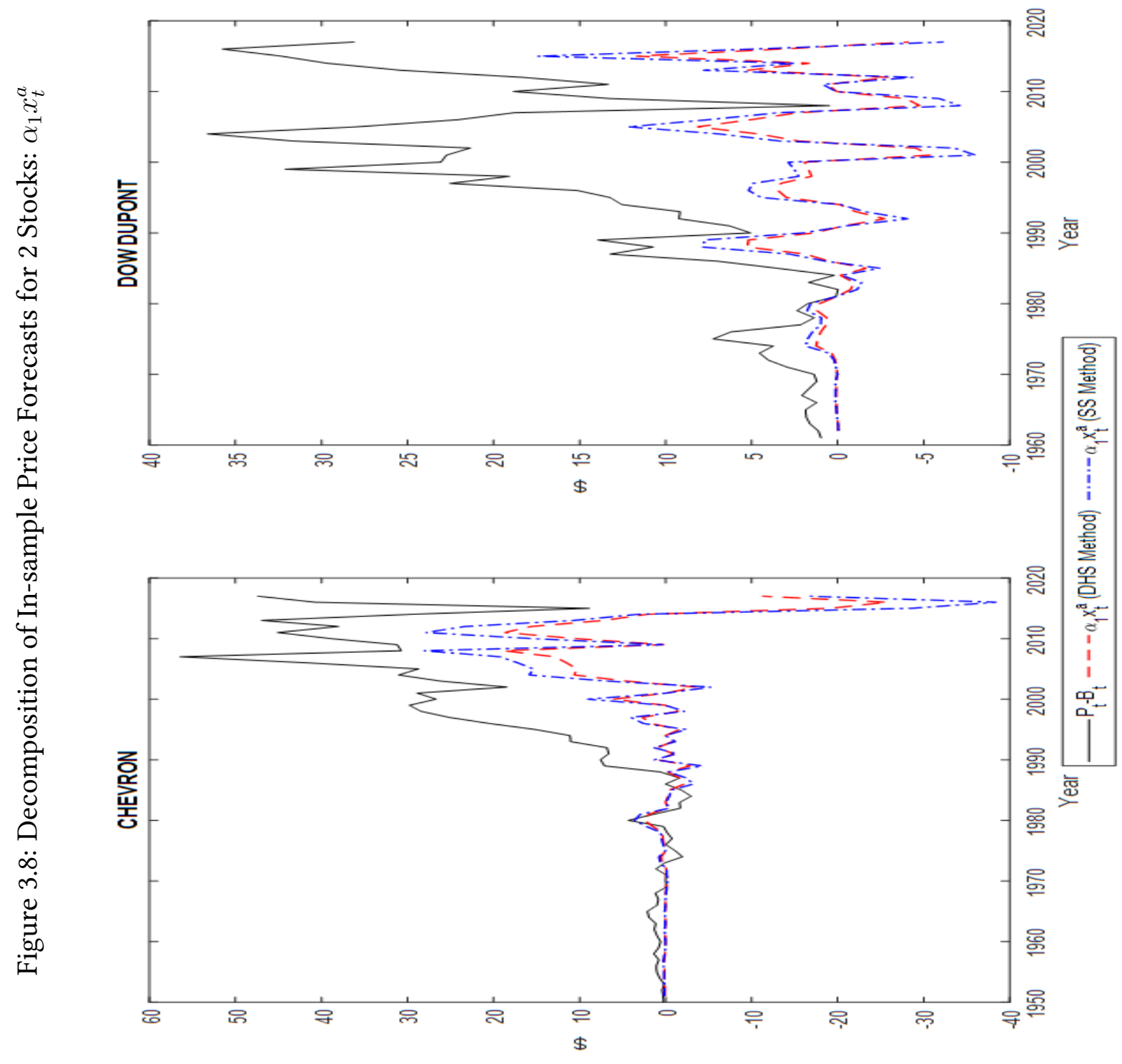




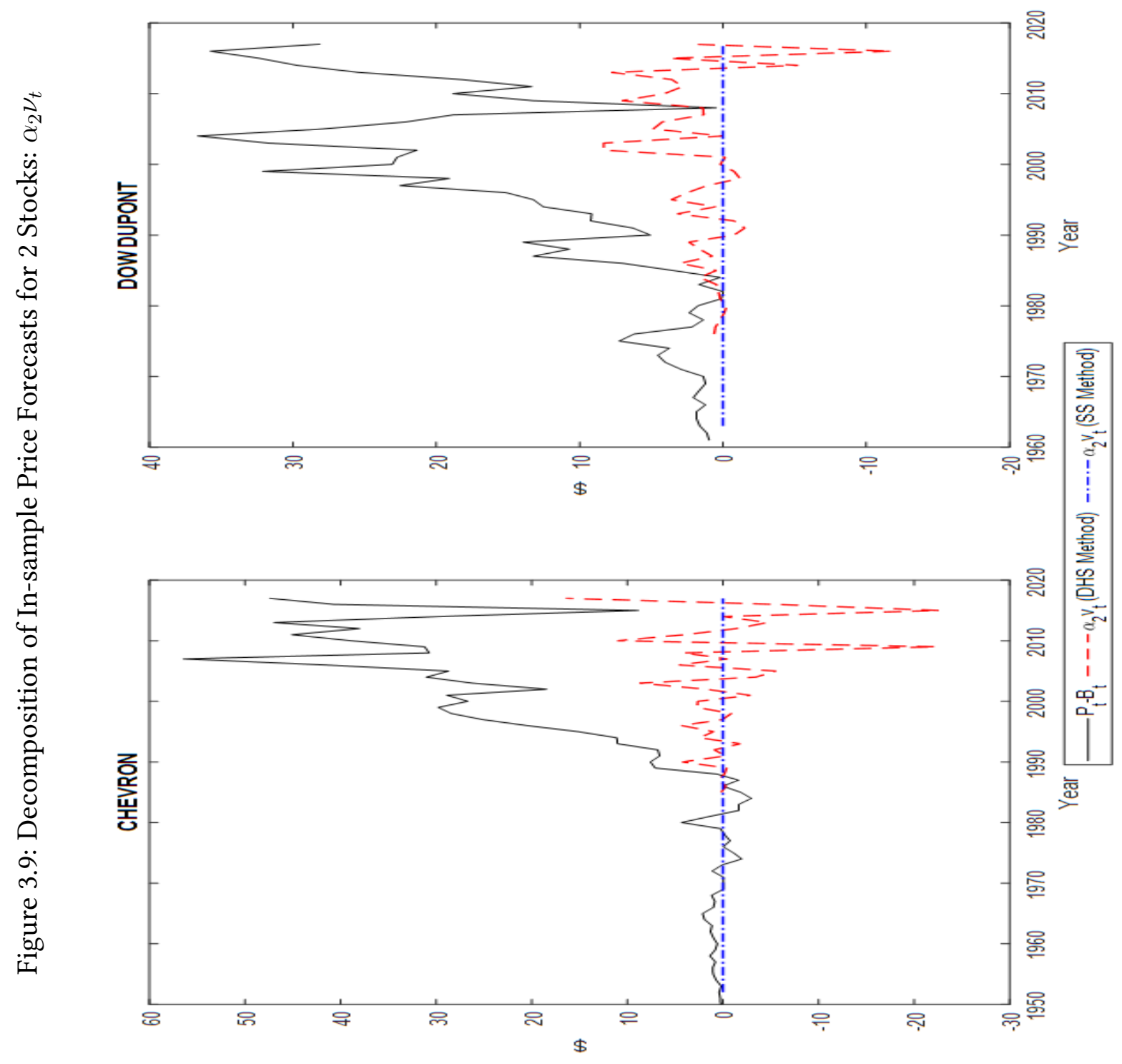




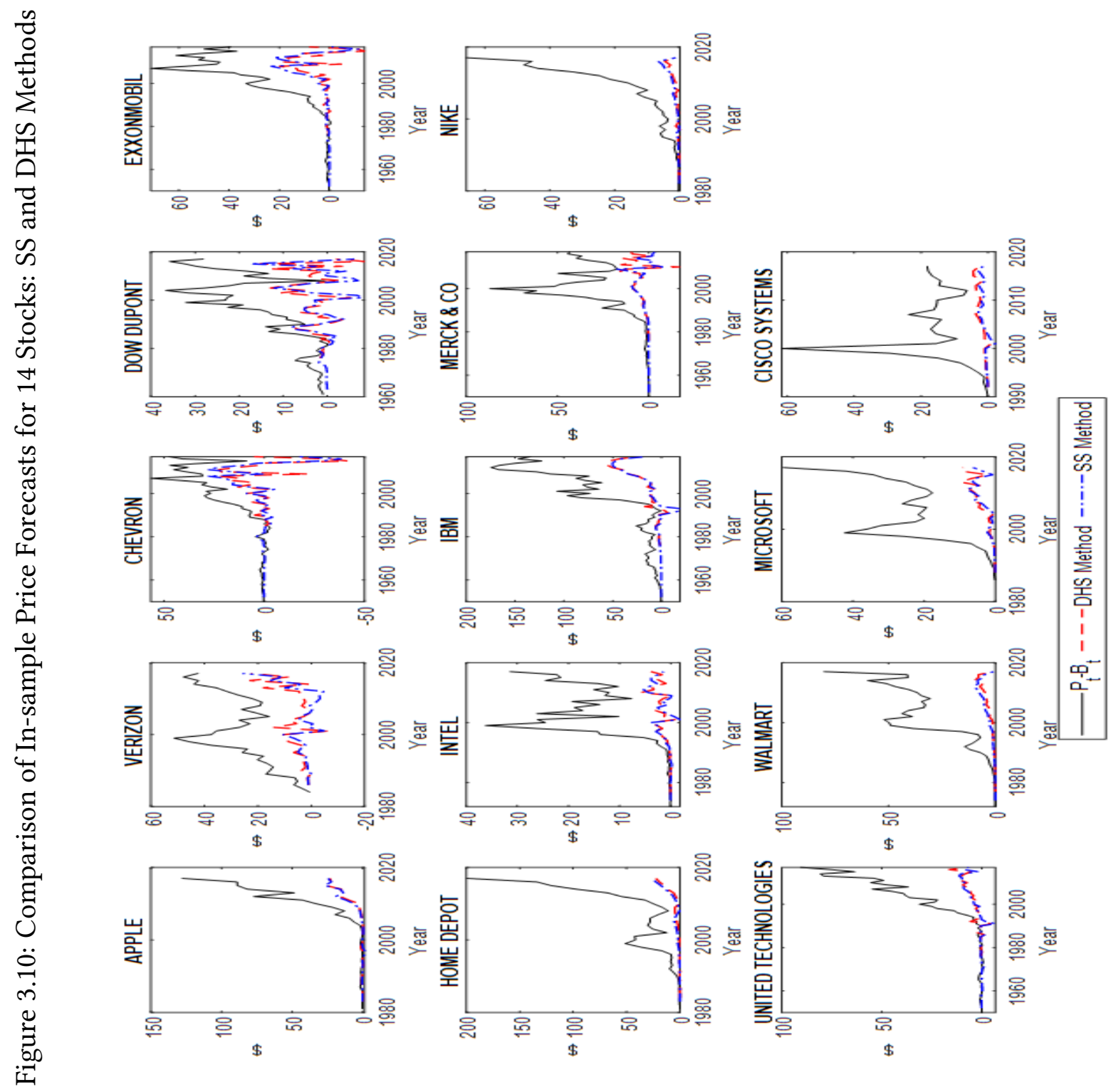




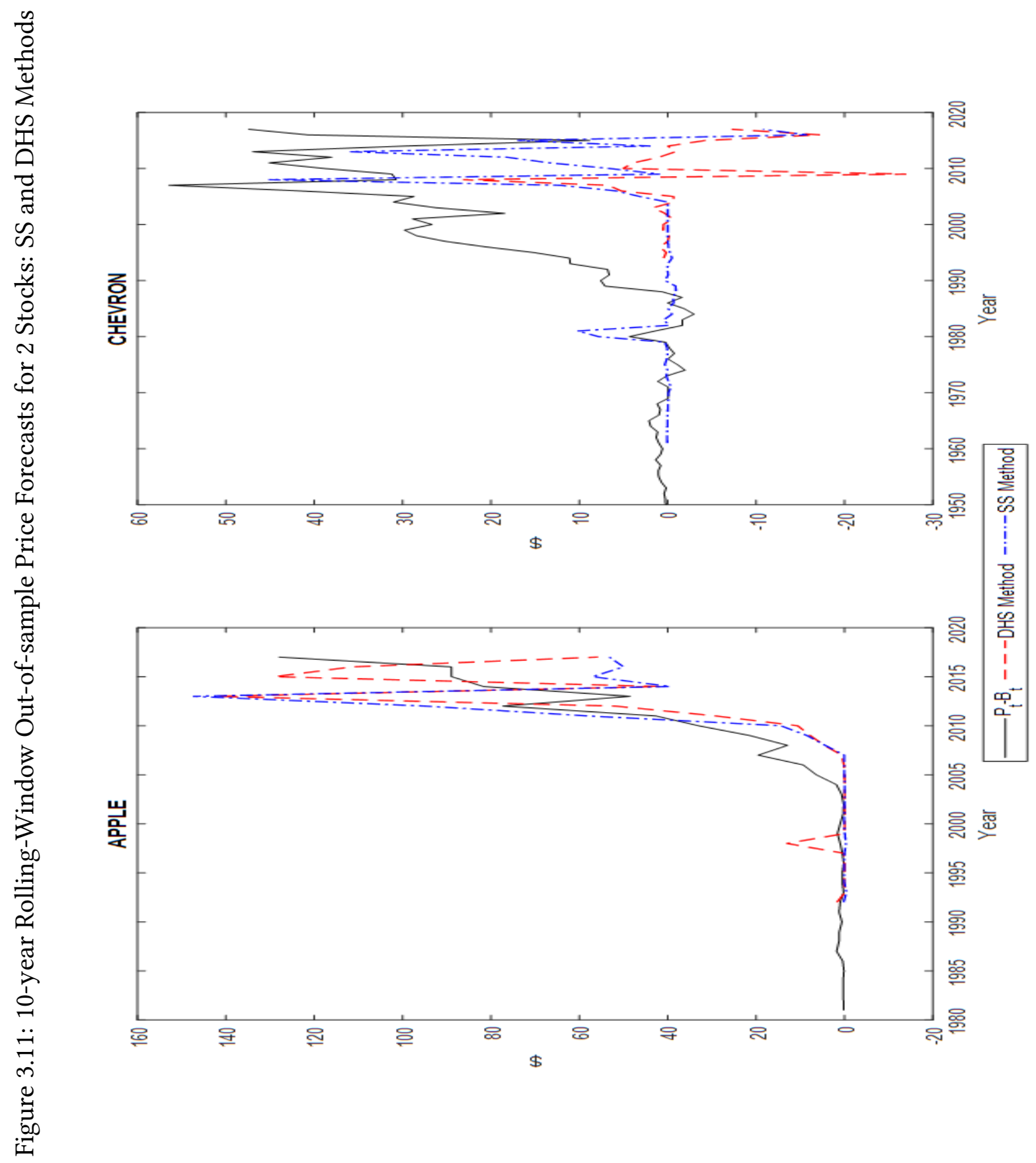




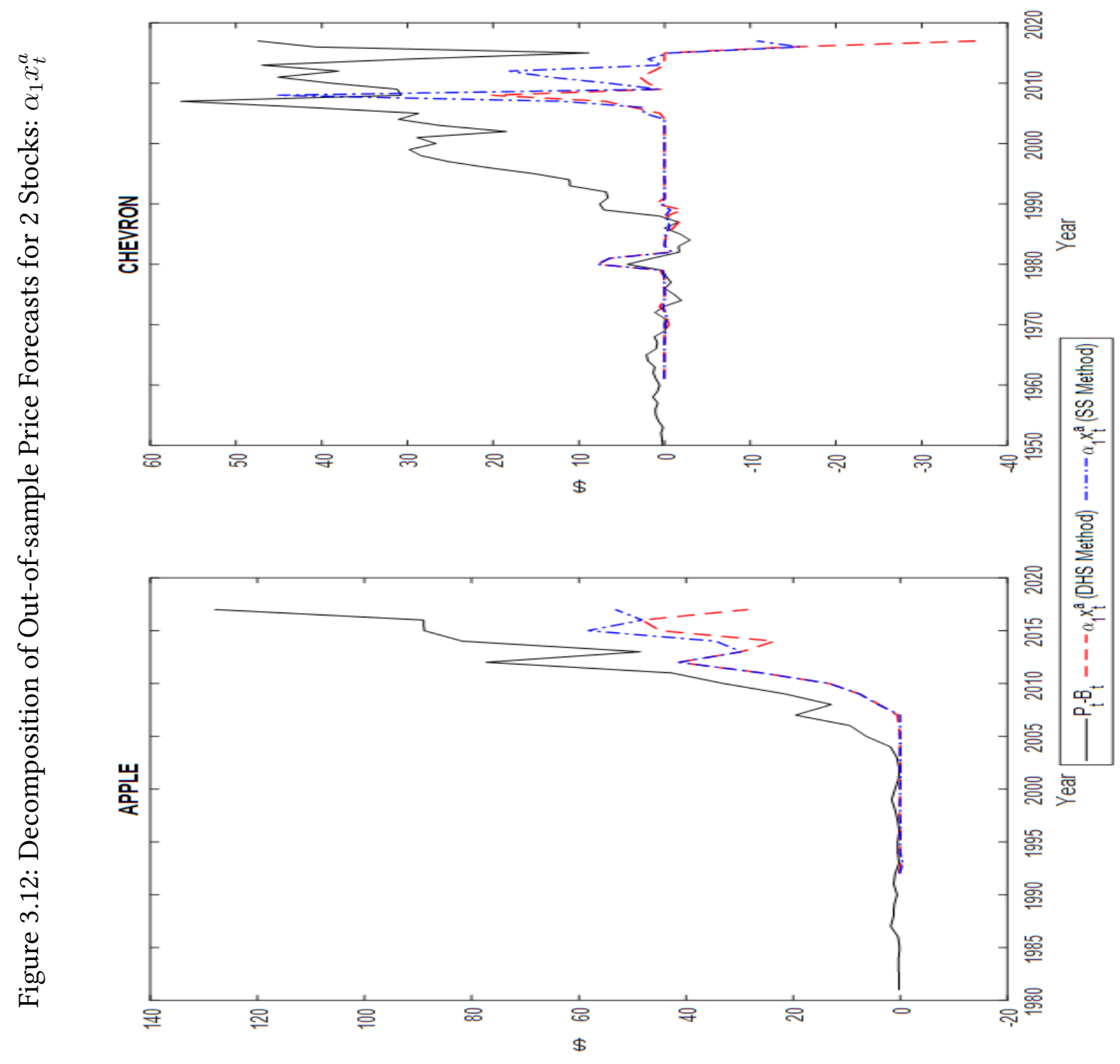




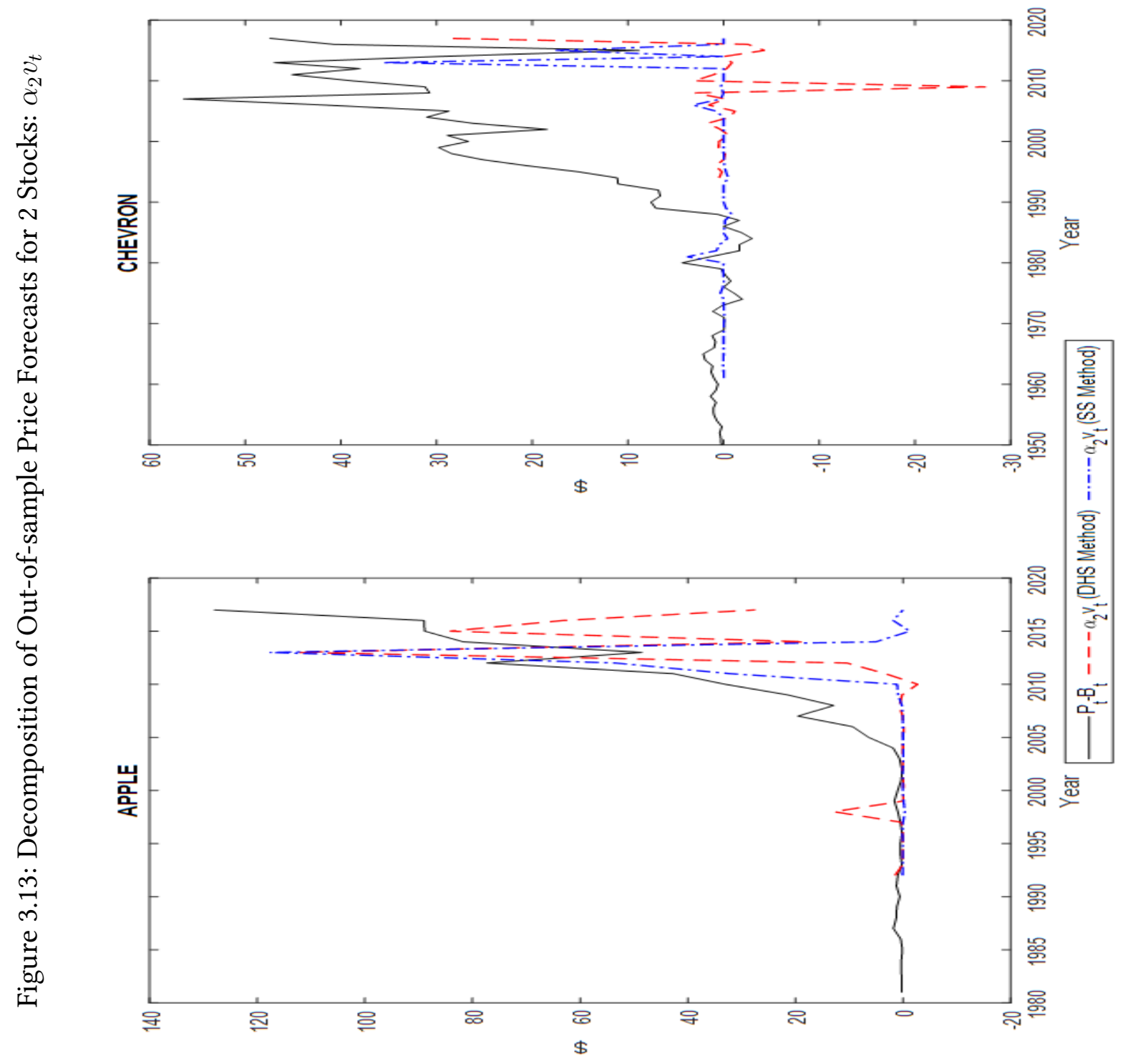




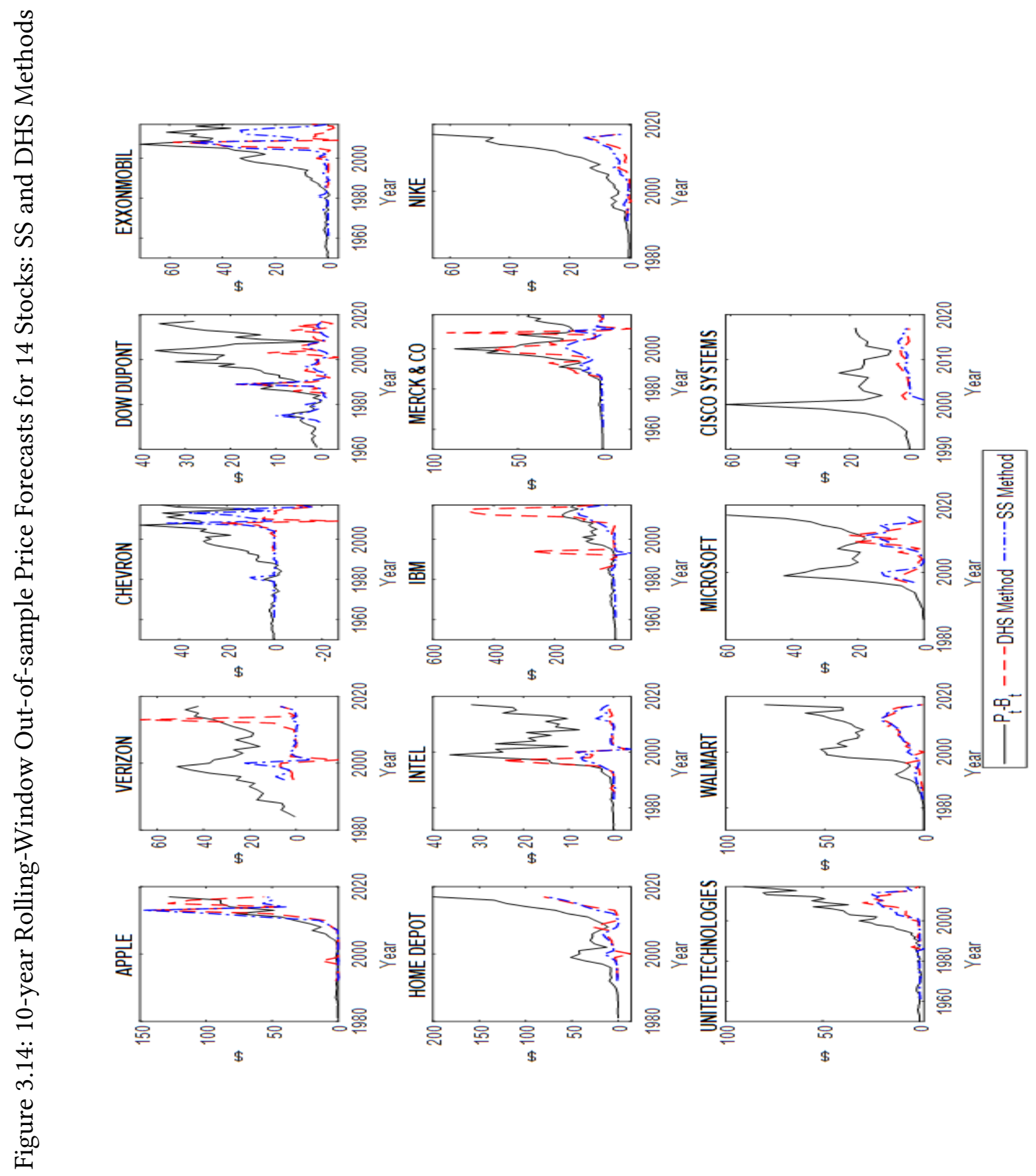




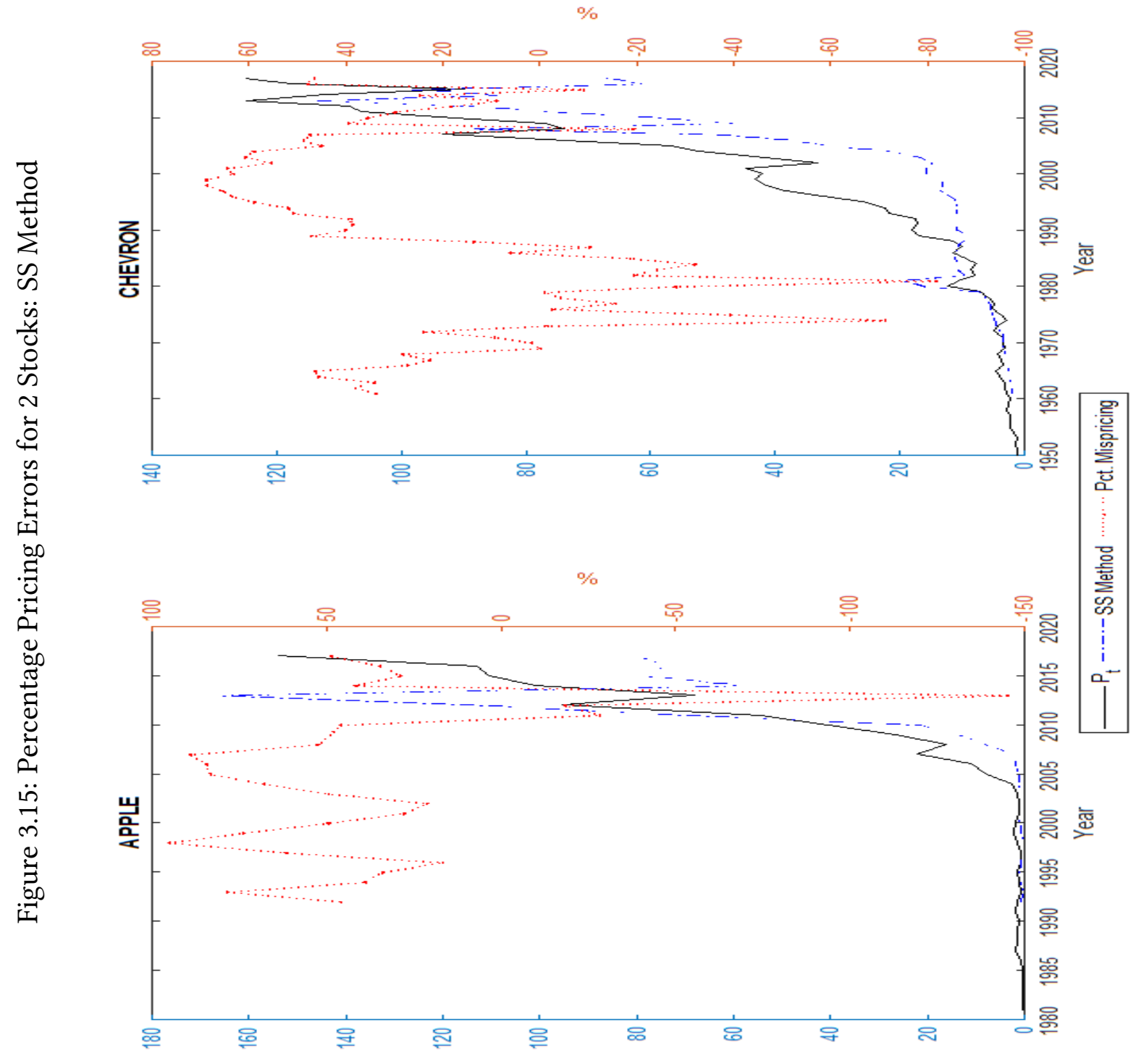




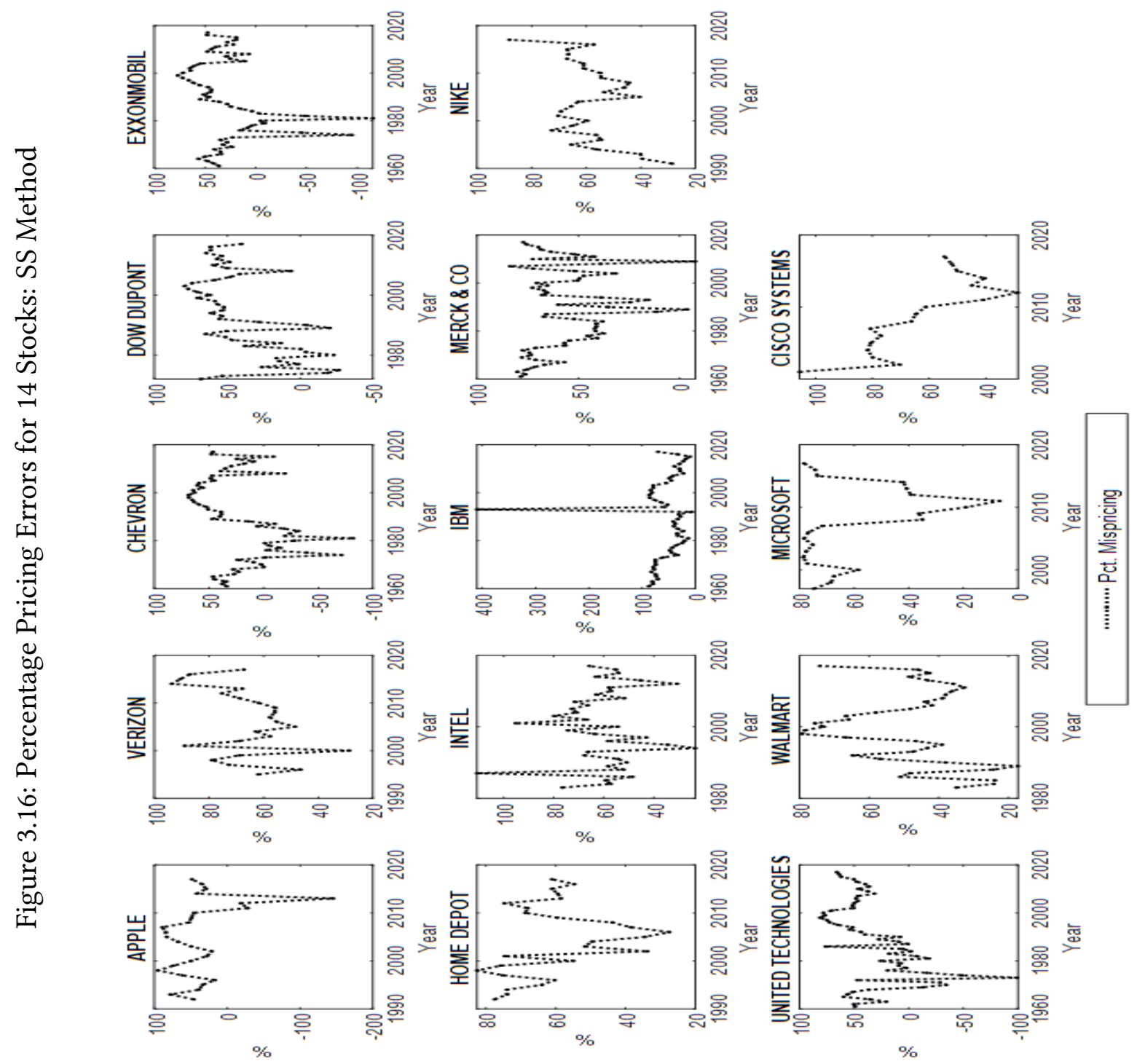




\subsection{Appendix}

\subsubsection{Alternative Representation of the State Space Model}

The dynamic evolution of abnormal earnings is specified in Equations (3.7a) and (3.7b) in the main text. Eqs. (3.7a) and (3.7b) can be represented in state space form in which the measurement (observation) equation relating $y_{t}$ to latent unobserved variable $\alpha_{t}$ is formulated as:

$$
\underset{n \times 1}{y_{t}}=\underset{n \times 1}{\omega y_{t-1}}+\underset{(n \times n)(n \times 1)}{z_{t}} \underset{n \times 1}{\alpha_{t}}+\underset{n+1}{\varepsilon_{t}}, \quad \varepsilon_{t} \sim i i d N\left(0, H_{t}\right)
$$

and the transition (state) equation describing the evolution of the latent variable can be formulated as:

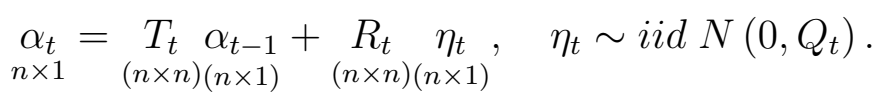

Here, $y_{t}$ is the column vector of observed abnormal earnings at time $t . \omega$ is the unknown persistence parameter of abnormal earnings appearing in Eq. (3.7a). $z_{t}=R_{t}=I_{n}$ is a $n$-dimensional identity matrix, $\alpha_{t}=v_{t}$, the 'other information variable' in the RIM, is the $n \times 1$ state vector, $H_{t}=\sigma_{\varepsilon}^{2} I_{n}$ is the covariance matrix for the measurement noise $\varepsilon_{t}, T_{t}=\gamma I_{n}$ is a matrix of parameters governing the state transition, and $Q_{t}=\sigma_{\eta}^{2} I_{n}$ is the covariance matrix for the signal shock $\eta_{t}$. $H_{t}$ and $Q_{t}$ are serially and mutually independent.

Given the linear Gaussian state space specification above, we estimate the unknown parameters contained in the vector, $\phi=\left\{\omega, \gamma, \sigma_{\varepsilon}^{2}, \sigma_{\eta}^{2}\right\}$, as well as the latent state variable $\alpha_{t}$, using the Kalman filter. 
Initialization. We initialize the Kalman filter with the initial state, $a_{t \mid t}$ and $p_{t \mid t}$ at $t=0$. Let $a_{t \mid t}=E\left[\alpha_{t} \mid Y_{t}\right]$, where $Y_{t}=\left\{y_{t}, y_{t-1}, y_{t-2}, \ldots y_{1}\right\}$, be the filter mean of unobserved state $a_{t}$, given observations up to and including $y_{t}$, and $p_{t \mid t}$ be the $n \times n$ covariance matrix $p_{t \mid t}=\operatorname{var}\left(\alpha_{t} \mid Y_{t}\right)$.

Prediction. Given $a_{t \mid t}$ and $p_{t \mid t}$, the one-step ahead predictor $a_{t+1 \mid t}$ is provided by:

$$
\begin{aligned}
a_{t+1 \mid t}= & E\left(\alpha_{t+1} \mid Y_{t}\right) \\
& =E\left(T_{t+1} \alpha_{t}+R_{t+1} \eta_{t+1} \mid Y_{t}\right) \\
& =T_{t+1} \cdot a_{t \mid t} \\
p_{t+1 \mid t}= & \operatorname{var}\left(\alpha_{t+1} \mid Y_{t}\right) \\
= & \operatorname{var}\left(T_{t+1} \alpha_{t}+R_{t+1} n_{t+1} \mid Y_{t}\right) \\
= & T_{t+1} p_{t \mid t} T_{t+1}^{\prime}+R_{t+1} Q_{t+1} R_{t+1}^{\prime}
\end{aligned}
$$

Filtering. Once a new observation $y_{t+1}$ arrives, the one-step ahead predictions are updated using the filtering equations:

$$
\begin{aligned}
& a_{t+1 \mid t+1}=a_{t+1}+\left(p_{t+1} z_{t+1}^{\prime} F_{t+1}^{-1}\right) \varphi_{t+1} \\
& p_{t+1 \mid t+1}=p_{t+1}-p_{t+1} z_{t+1} F_{t+1}^{-1} z_{t+1}^{\prime} p_{t+1}
\end{aligned}
$$

where $\varphi_{t+1}=y_{t+1}-z_{t+1} a_{t+1}-\omega y_{t}=y_{t+1}-E\left(y_{t+1} \mid Y_{t}\right)$ is the one-step ahead prediction error and $F_{t+1}=\operatorname{var}\left(\varphi_{t+1}\right)=z_{t+1} p_{t+1} z_{t+1}^{\prime}+H_{t+1}$. Eqs. (3.20) and (3.21) together represent the Kalman filter, whose application generates the filter and predictive means and variances of the state variables $\alpha_{t}$, conditional on $Y_{t}$ and $\phi=\left\{\omega, \gamma, \sigma_{\varepsilon}^{2}, \sigma_{\eta}^{2}\right\}$. 
Log-likelihood construction. As a by-product of the above recursive predictive and filtering equations, we can recursively compute $f\left(y_{t} \mid Y_{t-1}, \phi\right)$ for $t=1,2, \ldots, N$ from the normal probability density function. We can use these densities to construct the log-likelihood as follows:

$$
\log L_{j}=-\frac{N}{2} \log (2 \pi)-\frac{1}{2} \sum_{t=1}^{N} \log \left|F_{t}\right|-\frac{1}{2} \sum_{i=1}^{N} \varphi_{t}^{\prime} F_{t}^{-1} \varphi_{t}
$$

where $\left|F_{t}\right|$ is the determinant of $F_{t}$. We use numerical methods to minimize the negative of the log-likelihood function in Eq. (3.22), with respect to the unknown vector of parameters $\phi$. 
Table 3.15: Variable Description List

\begin{tabular}{|c|c|c|}
\hline $\begin{array}{l}\text { Variable } \\
\text { Name }\end{array}$ & Data Source & Variable Description \\
\hline $\begin{array}{l}\text { AJEX- } \\
\text { Adjustment } \\
\text { Factor (Com- } \\
\text { pany) } \\
\text { Cumulative } \\
\text { by Ex-Date }\end{array}$ & $\begin{array}{l}\text { CRSP/COMPUSTAT } \\
\text { Merged }\end{array}$ & $\begin{array}{l}\text { This item represents a ratio that enables } \\
\text { you to adjust per-share data (i.e., price, } \\
\text { earnings per share, and dividends per } \\
\text { share), as well as share data (i.e., shares } \\
\text { outstanding and shares traded) for all stock } \\
\text { splits and stock dividends that occur sub- } \\
\text { sequent to the end of a given period. The } \\
\text { cumulative adjustment factors for all pe- } \\
\text { riods are changed whenever a stock split } \\
\text { or stock dividend occurs. The factors are } \\
\text { carried to six decimal places to minimize } \\
\text { rounding errors. If for example, a two-for- } \\
\text { one split occurred in fiscal } 1994 \text {, the adjust- } \\
\text { ment factor for } 1993 \text { would be indicated as } \\
2.000000 \text {. If, in addition, a } 2 \text { percent stock } \\
\text { dividend occurred in } 1993 \text {, the adjustment } \\
\text { factor for } 1992 \text { would be indicated as cu- } \\
\text { mulative adjustment, } 2.040000 \text { ( } 2.000000 \text { X } \\
\text { 1.020000). The adjustment factors are indi- } \\
\text { cated as } 1.000000 \text { when no changes in cap- } \\
\text { determine the adjustment of per-share data. }\end{array}$ \\
\hline
\end{tabular}




\begin{tabular}{|c|c|c|}
\hline $\begin{array}{l}\text { BKVLPS- } \\
\text { Book Value } \\
\text { Per Share }\end{array}$ & $\begin{array}{l}\text { CRSP/COMPUSTAT } \\
\text { Merged }\end{array}$ & $\begin{array}{l}\text { For companies, Book Value Per Share is } \\
\text { based on fiscal year-end data and repre- } \\
\text { sents Common Equity Liquidation Value } \\
\text { (CEQL) divided by Common Shares Out- } \\
\text { standing (CSHO). For indexes, Book Value } \\
\text { Per Share is an annual figure calculated } \\
\text { from the calendar year-end index data. }\end{array}$ \\
\hline $\begin{array}{l}\text { CONM- } \\
\text { Company } \\
\text { Name }\end{array}$ & $\begin{array}{l}\text { CRSP/COMPUSTAT } \\
\text { Merged }\end{array}$ & $\begin{array}{l}\text { This is the official company name as re- } \\
\text { ported on its EDGAR SEC filings. }\end{array}$ \\
\hline CUSIP & $\begin{array}{l}\text { CRSP/COMPUSTAT } \\
\text { Merged }\end{array}$ & $\begin{array}{l}\text { CUSIP is the nine-character alphanumeric } \\
\text { code assigned by the CUSIP Service Bureau } \\
\text { to identify various securities. }\end{array}$ \\
\hline $\begin{array}{l}\text { I/B/E/S CUSIP } \\
\text { (8-digit) }\end{array}$ & $\mathrm{I} / \mathrm{B} / \mathrm{E} / \mathrm{S}$ & $\begin{array}{l}\text { The I/B/E/S CUSIP is an I/B/E/S conven- } \\
\text { tion that helps differentiate CUSIPs for U.S. } \\
\text { companies from SEDOLs for international } \\
\text { companies. The I/B/E/S CUSIP for U.S. } \\
\text { is the company's 8-character CUSIP. The } \\
\text { I/B/E/S CUSIP for international companies } \\
\text { is the 6-character SEDOL, preceded by a } \\
\text { two-digit country code. I/B/E/S CUSIPs do } \\
\text { not include the check sum digit. }\end{array}$ \\
\hline
\end{tabular}




\begin{tabular}{|c|c|c|}
\hline $\begin{array}{l}\text { EPSPX- } \\
\text { Earnings Per } \\
\text { Share (Basic) } \\
\text { Excluding } \\
\text { Extraordinary } \\
\text { Items }\end{array}$ & $\begin{array}{l}\text { CRSP/COMPUSTAT } \\
\text { Merged }\end{array}$ & $\begin{array}{l}\text { This item represents basic earnings per } \\
\text { share before extraordinary items and dis- } \\
\text { continued operations. }\end{array}$ \\
\hline $\begin{array}{l}\text { FPEDATS- } \\
\text { Forecast } \\
\text { Period End } \\
\text { Date }\end{array}$ & $\mathrm{I} / \mathrm{B} / \mathrm{E} / \mathrm{S}$ & $\begin{array}{l}\text { Forecast Period End Date (FPEDATS) is the } \\
\text { date to which the estimate applies. For } 70 \% \\
\text { of the companies, an estimate for a particu- } \\
\text { lar fiscal year will have an FPEDATS of De- } \\
\text { cember 31st of that year. }\end{array}$ \\
\hline $\begin{array}{l}\text { FYEAR-Data } \\
\text { Year - Fiscal }\end{array}$ & $\begin{array}{l}\text { CRSP/COMPUSTAT } \\
\text { Merged }\end{array}$ & $\begin{array}{l}\text { This item represents the fiscal year of the } \\
\text { current fiscal year-end month. If the cur- } \\
\text { rent fiscal year-end month falls in January } \\
\text { through May, this item is the current calen- } \\
\text { dar year minus } 1 \text { year. If the current fis- } \\
\text { cal year-end month falls in June through } \\
\text { December, this item is the current calendar } \\
\text { year. }\end{array}$ \\
\hline $\begin{array}{l}\text { LPERMCO- } \\
\text { Historical } \\
\text { CRSP } \\
\text { PERMCO } \\
\text { Link to COM- } \\
\text { PUSTAT } \\
\text { Record }\end{array}$ & $\begin{array}{l}\text { CRSP/COMPUSTAT } \\
\text { Merged }\end{array}$ & $\begin{array}{l}\text { CRSP PERMCO (company identifier) link } \\
\text { during link period. It is set to zero if there } \\
\text { is no CRSP link during the range. }\end{array}$ \\
\hline
\end{tabular}




\begin{tabular}{|c|c|c|}
\hline $\begin{array}{l}\text { LPERMNO- } \\
\text { Historical } \\
\text { CRSP } \\
\text { PERMNO } \\
\text { Link to COM- } \\
\text { PUSTAT } \\
\text { Record }\end{array}$ & $\begin{array}{l}\text { CRSP/COMPUSTAT } \\
\text { Merged }\end{array}$ & $\begin{array}{l}\text { CRSP PERMNO link (company identifier) } \\
\text { during link period. It is set to zero if there } \\
\text { is no CRSP link during the range. }\end{array}$ \\
\hline $\begin{array}{l}\text { MEANEST- } \\
\text { Mean Esti- } \\
\text { mate }\end{array}$ & $\mathrm{I} / \mathrm{B} / \mathrm{E} / \mathrm{S}$ & $\begin{array}{l}\text { The average of all estimates from all ana- } \\
\text { lysts for a given issue and time period. }\end{array}$ \\
\hline $\begin{array}{l}\text { NAICS-North } \\
\text { American } \\
\text { Industry } \\
\text { Classification } \\
\text { Code }\end{array}$ & $\begin{array}{l}\text { CRSP/COMPUSTAT } \\
\text { Merged }\end{array}$ & $\begin{array}{l}\text { NAICS is a hierarchical structure and can } \\
\text { consist of up to six digits/levels. The first } \\
\text { two digits of the structure designate the } \\
\text { NAICS sectors that represent general cat- } \\
\text { egories of economic activity. The third } \\
\text { digit designates the subsector, the fourth } \\
\text { digit designates the industry group, the } \\
\text { fifth digit designates the NAICS industry, } \\
\text { and the sixth digit designates the national } \\
\text { industry. }\end{array}$ \\
\hline $\begin{array}{l}\text { NCUSIP } \\
\text { (8-digit) }\end{array}$ & CRSP & $\begin{array}{l}\text { NCUSIP is the } 8 \text {-digit historical CUSIP in } \\
\text { CRSP data. NCUSIP is analogous to I/B/E/S' } \\
\text { CUSIP. }\end{array}$ \\
\hline $\begin{array}{l}\text { PRCC_C- } \\
\text { Price Close } \\
\text { - Annual - } \\
\text { Calendar }\end{array}$ & $\begin{array}{l}\text { CRSP/COMPUSTAT } \\
\text { Merged }\end{array}$ & $\begin{array}{l}\text { This item is the closing market price at the } \\
\text { calendar year end. }\end{array}$ \\
\hline
\end{tabular}




\begin{tabular}{|c|c|c|}
\hline $\begin{array}{l}\text { PRCC_F- } \\
\text { Price Close } \\
\text { - Annual - } \\
\text { Fiscal }\end{array}$ & $\begin{array}{l}\text { CRSP/COMPUSTAT } \\
\text { Merged }\end{array}$ & $\begin{array}{l}\text { This item is the closing market price at the } \\
\text { fiscal year-end. }\end{array}$ \\
\hline $\begin{array}{l}\text { STATPERS- } \\
\text { IBES Statisti- } \\
\text { cal Period }\end{array}$ & $\mathrm{I} / \mathrm{B} / \mathrm{E} / \mathrm{S}$ & $\begin{array}{l}\mathrm{I} / \mathrm{B} / \mathrm{E} / \mathrm{S} \text { statistical period is the date when } \\
\text { the set of summary statistics was calcu- } \\
\text { lated. }\end{array}$ \\
\hline $\begin{array}{l}\text { TIC-Ticker } \\
\text { Symbol }\end{array}$ & $\begin{array}{l}\text { CRSP/COMPUSTAT } \\
\text { Merged }\end{array}$ & $\begin{array}{l}\text { This item identifies the symbol associated } \\
\text { with trading of a particular issue on an ex- } \\
\text { change. }\end{array}$ \\
\hline
\end{tabular}


Table 3.16: Index Constituents List

\begin{tabular}{|c|c|c|c|c|}
\hline Company Name & $\begin{array}{l}\text { Ticker Sym- } \\
\text { bol }\end{array}$ & $\begin{array}{l}\text { DJIA } \\
\text { Con- } \\
\text { stituent }\end{array}$ & $\begin{array}{l}\text { S\&P } 500 \\
\text { Con- } \\
\text { stituent }\end{array}$ & $\begin{array}{l}\text { Used in } \\
\text { Sample }\end{array}$ \\
\hline $\begin{array}{l}\text { AGILENT TECHNOLOGIES } \\
\text { INC }\end{array}$ & A & No & Yes & Yes \\
\hline $\begin{array}{l}\text { AMERICAN AIRLINES } \\
\text { GROUP INC }\end{array}$ & AAL & No & Yes & No \\
\hline $\begin{array}{l}\text { ADVANCE AUTO PARTS } \\
\text { INC }\end{array}$ & AAP & No & Yes & Yes \\
\hline APPLE INC & AAPL & Yes & Yes & Yes \\
\hline ABBVIE INC & ABBV & No & Yes & Yes \\
\hline $\begin{array}{l}\text { AMERISOURCEBERGEN } \\
\text { CORP }\end{array}$ & $\mathrm{ABC}$ & No & Yes & No \\
\hline ABIOMED INC & $\mathrm{ABMD}$ & No & Yes & No \\
\hline ABBOTT LABORATORIES & $\mathrm{ABT}$ & No & Yes & Yes \\
\hline ACCENTURE PLC & $\mathrm{ACN}$ & No & Yes & No \\
\hline ADOBE INC & $\mathrm{ADBE}$ & No & Yes & Yes \\
\hline ANALOG DEVICES & $\mathrm{ADI}$ & No & Yes & No \\
\hline $\begin{array}{l}\text { ARCHER-DANIELS- } \\
\text { MIDLAND CO }\end{array}$ & $\mathrm{ADM}$ & No & Yes & Yes \\
\hline $\begin{array}{l}\text { AUTOMATIC DATA PRO- } \\
\text { CESSING }\end{array}$ & $\mathrm{ADP}$ & No & Yes & Yes \\
\hline
\end{tabular}




\begin{tabular}{|c|c|c|c|c|}
\hline $\begin{array}{l}\text { ALLIANCE DATA SYSTEMS } \\
\text { CORP }\end{array}$ & ADS & No & Yes & Yes \\
\hline AUTODESK INC & ADSK & No & Yes & No \\
\hline AMEREN CORP & AEE & No & Yes & No \\
\hline $\begin{array}{l}\text { AMERICAN ELECTRIC } \\
\text { POWER CO }\end{array}$ & $\mathrm{AEP}$ & No & Yes & No \\
\hline AES CORP & AES & No & Yes & No \\
\hline AFLAC INC & AFL & No & Yes & No \\
\hline ALLERGAN PLC & AGN & No & Yes & Yes \\
\hline $\begin{array}{l}\text { AMERICAN INTERNA- } \\
\text { TIONAL GROUP }\end{array}$ & AIG & No & Yes & Yes \\
\hline $\begin{array}{l}\text { APARTMENT INVST \& } \\
\text { MGMT CO }\end{array}$ & AIV & No & Yes & Yes \\
\hline ASSURANT INC & AIZ & No & Yes & Yes \\
\hline $\begin{array}{l}\text { ARTHUR J GALLAGHER \& } \\
\text { CO }\end{array}$ & AJG & No & Yes & Yes \\
\hline $\begin{array}{l}\text { AKAMAI TECHNOLOGIES } \\
\text { INC }\end{array}$ & AKAM & No & Yes & No \\
\hline ALBEMARLE CORP & ALB & No & Yes & Yes \\
\hline ALIGN TECHNOLOGY INC & ALGN & No & Yes & Yes \\
\hline ALASKA AIR GROUP INC & ALK & No & Yes & No \\
\hline ALLSTATE CORP & ALL & No & Yes & Yes \\
\hline ALLEGION PLC & ALLE & No & Yes & No \\
\hline
\end{tabular}




\begin{tabular}{|c|c|c|c|c|}
\hline $\begin{array}{l}\text { ALEXION PHARMACEUTI- } \\
\text { CALS INC }\end{array}$ & ALXN & No & Yes & Yes \\
\hline APPLIED MATERIALS INC & AMAT & No & Yes & Yes \\
\hline $\begin{array}{l}\text { ADVANCED MICRO DE- } \\
\text { VICES }\end{array}$ & AMD & No & Yes & No \\
\hline AMETEK INC & AME & No & Yes & Yes \\
\hline $\begin{array}{l}\text { AFFILIATED MANAGERS } \\
\text { GRP INC }\end{array}$ & AMG & No & Yes & Yes \\
\hline AMGEN INC & AMGN & No & Yes & Yes \\
\hline $\begin{array}{l}\text { AMERIPRISE FINANCIAL } \\
\text { INC }\end{array}$ & AMP & No & Yes & Yes \\
\hline AMERICAN TOWER CORP & AMT & No & Yes & Yes \\
\hline AMAZON.COM INC & AMZN & No & Yes & No \\
\hline ARISTA NETWORKS INC & ANET & No & Yes & Yes \\
\hline ANSYS INC & ANSS & No & Yes & Yes \\
\hline ANTHEM INC & ANTM & No & Yes & Yes \\
\hline AON PLC & $\mathrm{AON}$ & No & Yes & Yes \\
\hline SMITH (A.O.) & AOS & No & Yes & No \\
\hline APACHE CORP & APA & No & Yes & Yes \\
\hline $\begin{array}{l}\text { ANADARKO PETROLEUM } \\
\text { CORP }\end{array}$ & APC & No & Yes & Yes \\
\hline $\begin{array}{l}\text { AIR PRODUCTS \& CHEMI- } \\
\text { CALS INC }\end{array}$ & APD & No & Yes & Yes \\
\hline
\end{tabular}




\begin{tabular}{|c|c|c|c|c|}
\hline AMPHENOL CORP & $\mathrm{APH}$ & No & Yes & No \\
\hline APTIV PLC & APTV & No & Yes & No \\
\hline $\begin{array}{l}\text { ALEXANDRIA R E EQUI- } \\
\text { TIES INC }\end{array}$ & ARE & No & Yes & No \\
\hline ARCONIC INC & ARNC & No & Yes & No \\
\hline ATMOS ENERGY CORP & ATO & No & Yes & No \\
\hline $\begin{array}{l}\text { ACTIVISION BLIZZARD } \\
\text { INC }\end{array}$ & ATVI & No & Yes & Yes \\
\hline $\begin{array}{l}\text { AVALONBAY COMMUNI- } \\
\text { TIES INC }\end{array}$ & AVB & No & Yes & No \\
\hline BROADCOM INC & AVGO & No & Yes & Yes \\
\hline AVERY DENNISON CORP & AVY & No & Yes & Yes \\
\hline $\begin{array}{l}\text { AMERICAN WATER } \\
\text { WORKS CO INC }\end{array}$ & AWK & No & Yes & Yes \\
\hline AMERICAN EXPRESS CO & AXP & Yes & Yes & Yes \\
\hline AUTOZONE INC & $\mathrm{AZO}$ & No & Yes & No \\
\hline BOEING CO & $\mathrm{BA}$ & Yes & Yes & No \\
\hline BANK OF AMERICA CORP & $\mathrm{BAC}$ & No & Yes & Yes \\
\hline $\begin{array}{l}\text { BAXTER INTERNATIONAL } \\
\text { INC }\end{array}$ & BAX & No & Yes & Yes \\
\hline BB\&T CORP & $\mathrm{BBT}$ & No & Yes & Yes \\
\hline BEST BUY CO INC & BBY & No & Yes & Yes \\
\hline BECTON DICKINSON \& CO & BDX & No & Yes & No \\
\hline
\end{tabular}




\begin{tabular}{|c|c|c|c|c|}
\hline $\begin{array}{l}\text { FRANKLIN RESOURCES } \\
\text { INC }\end{array}$ & BEN & No & Yes & Yes \\
\hline BROWN FORMAN CORP & BF.B & No & Yes & No \\
\hline $\begin{array}{l}\text { BRIGHTHOUSE FINANL } \\
\text { INC }\end{array}$ & $\mathrm{BHF}$ & No & Yes & No \\
\hline BAKER HUGHES A GE CO & BHGE & No & Yes & No \\
\hline BIOGEN INC & BIIB & No & Yes & Yes \\
\hline $\begin{array}{l}\text { BANK OF NEW YORK MEL- } \\
\text { LON CORP }\end{array}$ & $\mathrm{BK}$ & No & Yes & Yes \\
\hline BOOKING HOLDINGS INC & $\mathrm{BKNG}$ & No & Yes & No \\
\hline BLACKROCK INC & BLK & No & Yes & Yes \\
\hline BALL CORP & BLL & No & Yes & No \\
\hline $\begin{array}{l}\text { BRISTOL-MYERS SQUIBB } \\
\text { CO }\end{array}$ & BMY & No & Yes & Yes \\
\hline $\begin{array}{l}\text { BROADRIDGE FINANCIAL } \\
\text { SOLUTNS }\end{array}$ & $\mathrm{BR}$ & No & Yes & No \\
\hline BERKSHIRE HATHAWAY & BRK.B & No & Yes & No \\
\hline BOSTON SCIENTIFIC CORP & BSX & No & Yes & Yes \\
\hline BORGWARNER INC & BWA & No & Yes & Yes \\
\hline BOSTON PROPERTIES INC & $\mathrm{BXP}$ & No & Yes & Yes \\
\hline CITIGROUP INC & $\mathrm{C}$ & No & Yes & Yes \\
\hline CONAGRA BRANDS INC & CAG & No & Yes & Yes \\
\hline CARDINAL HEALTH INC & $\mathrm{CAH}$ & No & Yes & No \\
\hline
\end{tabular}




\begin{tabular}{|c|c|c|c|c|}
\hline CATERPILLAR INC & CAT & Yes & Yes & No \\
\hline CHUBB LTD & $\mathrm{CB}$ & No & Yes & Yes \\
\hline $\begin{array}{l}\text { CBOE GLOBAL MARKETS } \\
\text { INC }\end{array}$ & CBOE & No & Yes & Yes \\
\hline CBRE GROUP INC & CBRE & No & Yes & Yes \\
\hline CBS CORP & CBS & No & Yes & No \\
\hline $\begin{array}{l}\text { CROWN CASTLE INTL } \\
\text { CORP }\end{array}$ & $\mathrm{CCI}$ & No & Yes & No \\
\hline $\begin{array}{l}\text { CARNIVAL CORP/PLC } \\
\text { (USA) }\end{array}$ & $\mathrm{CCL}$ & No & Yes & Yes \\
\hline $\begin{array}{l}\text { CADENCE DESIGN SYS- } \\
\text { TEMS INC }\end{array}$ & CDNS & No & Yes & Yes \\
\hline CELANESE CORP & $\mathrm{CE}$ & No & Yes & Yes \\
\hline CELGENE CORP & CELG & No & Yes & No \\
\hline CERNER CORP & CERN & No & Yes & Yes \\
\hline $\begin{array}{l}\text { CF INDUSTRIES HOLDINGS } \\
\text { INC }\end{array}$ & $\mathrm{CF}$ & No & Yes & Yes \\
\hline $\begin{array}{l}\text { CITIZENS FINANCIAL } \\
\text { GROUP INC }\end{array}$ & CFG & No & Yes & Yes \\
\hline CHURCH \& DWIGHT INC & $\mathrm{CHD}$ & No & Yes & Yes \\
\hline $\begin{array}{l}\text { C H ROBINSON WORLD- } \\
\text { WIDE INC }\end{array}$ & CHRW & No & Yes & Yes \\
\hline $\begin{array}{l}\text { CHARTER COMMUNICA- } \\
\text { TIONS INC }\end{array}$ & CHTR & No & Yes & No \\
\hline
\end{tabular}




\begin{tabular}{|c|c|c|c|c|}
\hline CIGNA CORP & $\mathrm{CI}$ & No & Yes & Yes \\
\hline $\begin{array}{l}\text { CINCINNATI FINANCIAL } \\
\text { CORP }\end{array}$ & CINF & No & Yes & Yes \\
\hline COLGATE-PALMOLIVE CO & $\mathrm{CL}$ & No & Yes & No \\
\hline CLOROX CO/DE & CLX & No & Yes & No \\
\hline COMERICA INC & CMA & No & Yes & Yes \\
\hline COMCAST CORP & CMCSA & No & Yes & No \\
\hline CME GROUP INC & CME & No & Yes & Yes \\
\hline $\begin{array}{l}\text { CHIPOTLE MEXICAN } \\
\text { GRILL INC }\end{array}$ & CMG & No & Yes & No \\
\hline CUMMINS INC & CMI & No & Yes & No \\
\hline CMS ENERGY CORP & CMS & No & Yes & No \\
\hline CENTENE CORP & $\mathrm{CNC}$ & No & Yes & Yes \\
\hline $\begin{array}{l}\text { CENTERPOINT ENERGY } \\
\text { INC }\end{array}$ & $\mathrm{CNP}$ & No & Yes & No \\
\hline $\begin{array}{l}\text { CAPITAL ONE FINANCIAL } \\
\text { CORP }\end{array}$ & $\mathrm{COF}$ & No & Yes & Yes \\
\hline CABOT OIL \& GAS CORP & COG & No & Yes & Yes \\
\hline COOPER COMPANIES INC & $\mathrm{COO}$ & No & Yes & No \\
\hline CONOCOPHILLIPS & $\mathrm{COP}$ & No & Yes & No \\
\hline $\begin{array}{l}\text { COSTCO WHOLESALE } \\
\text { CORP }\end{array}$ & COsT & No & Yes & Yes \\
\hline COTY INC & COTY & No & Yes & Yes \\
\hline
\end{tabular}




\begin{tabular}{|c|c|c|c|c|}
\hline CAMPBELL SOUP CO & $\mathrm{CPB}$ & No & Yes & No \\
\hline CAPRI HOLDINGS LTD & CPRI & No & Yes & Yes \\
\hline COPART INC & CPRT & No & Yes & Yes \\
\hline SALESFORCE.COM INC & CRM & No & Yes & Yes \\
\hline CISCO SYSTEMS INC & $\mathrm{CSCO}$ & Yes & Yes & Yes \\
\hline CSX CORP & $\operatorname{cs} \mathrm{X}$ & No & Yes & No \\
\hline CINTAS CORP & CTAS & No & Yes & Yes \\
\hline CENTURYLINK INC & CTL & No & Yes & Yes \\
\hline $\begin{array}{l}\text { COGNIZANT TECH SOLU- } \\
\text { TIONS }\end{array}$ & CTSH & No & Yes & Yes \\
\hline CITRIX SYSTEMS INC & CTXS & No & Yes & Yes \\
\hline CVS HEALTH CORP & CVS & No & Yes & No \\
\hline CHEVRON CORP & CVX & Yes & Yes & Yes \\
\hline CONCHO RESOURCES INC & $\mathrm{CXO}$ & No & Yes & Yes \\
\hline DOMINION ENERGY INC & $\mathrm{D}$ & No & Yes & Yes \\
\hline DELTA AIR LINES INC & DAL & No & Yes & No \\
\hline DEERE \& CO & $\mathrm{DE}$ & No & Yes & No \\
\hline $\begin{array}{l}\text { DISCOVER FINANCIAL } \\
\text { SVCS }\end{array}$ & DFS & No & Yes & Yes \\
\hline DOLLAR GENERAL CORP & DG & No & Yes & No \\
\hline QUEST DIAGNOSTICS INC & DGX & No & Yes & Yes \\
\hline D R HORTON INC & DHI & No & Yes & Yes \\
\hline
\end{tabular}




\begin{tabular}{|c|c|c|c|c|}
\hline DANAHER CORP & DHR & No & Yes & No \\
\hline DISNEY (WALT) CO & DIS & Yes & Yes & No \\
\hline DISCOVERY INC & DISCA & No & Yes & No \\
\hline DISH NETWORK CORP & DISH & No & Yes & No \\
\hline $\begin{array}{l}\text { DIGITAL REALTY TRUST } \\
\text { INC }\end{array}$ & DLR & No & Yes & No \\
\hline DOLLAR TREE INC & DLTR & No & Yes & Yes \\
\hline DOVER CORP & DOV & No & Yes & No \\
\hline DUKE REALTY CORP & DRE & No & Yes & No \\
\hline $\begin{array}{l}\text { DARDEN RESTAURANTS } \\
\text { INC }\end{array}$ & DRI & No & Yes & No \\
\hline DTE ENERGY CO & DTE & No & Yes & Yes \\
\hline DUKE ENERGY CORP & DUK & No & Yes & Yes \\
\hline DAVITA INC & DVA & No & Yes & Yes \\
\hline DEVON ENERGY CORP & DVN & No & Yes & Yes \\
\hline DOWDUPONT INC & DWDP & Yes & Yes & Yes \\
\hline $\begin{array}{l}\text { DXC TECHNOLOGY COM- } \\
\text { PANY }\end{array}$ & DXC & No & Yes & No \\
\hline ELECTRONIC ARTS INC & EA & No & Yes & Yes \\
\hline EBAY INC & EBAY & No & Yes & Yes \\
\hline ECOLAB INC & ECL & No & Yes & Yes \\
\hline $\begin{array}{l}\text { CONSOLIDATED EDISON } \\
\text { INC }\end{array}$ & $\mathrm{ED}$ & No & Yes & No \\
\hline
\end{tabular}




\begin{tabular}{|c|c|c|c|c|}
\hline EQUIFAX INC & EFX & No & Yes & Yes \\
\hline EDISON INTERNATIONAL & EIX & No & Yes & No \\
\hline $\begin{array}{l}\text { LAUDER (ESTEE) COS INC - } \\
\text { CL A }\end{array}$ & $\mathrm{EL}$ & No & Yes & Yes \\
\hline EASTMAN CHEMICAL CO & EMN & No & Yes & Yes \\
\hline EMERSON ELECTRIC CO & EMR & No & Yes & Yes \\
\hline EOG RESOURCES INC & EOG & No & Yes & Yes \\
\hline EQUINIX INC & EQIX & No & Yes & Yes \\
\hline EQUITY RESIDENTIAL & EQR & No & Yes & No \\
\hline EVERSOURCE ENERGY & ES & No & Yes & Yes \\
\hline ESSEX PROPERTY TRUST & ESS & No & Yes & No \\
\hline $\begin{array}{l}\text { E TRADE FINANCIAL } \\
\text { CORP }\end{array}$ & ETFC & No & Yes & Yes \\
\hline EATON CORP PLC & ETN & No & Yes & No \\
\hline ENTERGY CORP & ETR & No & Yes & No \\
\hline EVERGY INC & EVRG & No & Yes & No \\
\hline $\begin{array}{l}\text { EDWARDS LIFESCIENCES } \\
\text { CORP }\end{array}$ & EW & No & Yes & No \\
\hline EXELON CORP & EXC & No & Yes & No \\
\hline $\begin{array}{l}\text { EXPEDITORS INTL WASH } \\
\text { INC }\end{array}$ & EXPD & No & Yes & Yes \\
\hline EXPEDIA GROUP INC & EXPE & No & Yes & No \\
\hline
\end{tabular}




\begin{tabular}{|c|c|c|c|c|}
\hline $\begin{array}{l}\text { EXTRA SPACE STORAGE } \\
\text { INC }\end{array}$ & EXR & No & Yes & Yes \\
\hline FORD MOTOR CO & $\mathrm{F}$ & No & Yes & No \\
\hline $\begin{array}{l}\text { DIAMONDBACK ENERGY } \\
\text { INC }\end{array}$ & FANG & No & Yes & Yes \\
\hline FASTENAL CO & FAST & No & Yes & Yes \\
\hline FACEBOOK INC & FB & No & Yes & Yes \\
\hline $\begin{array}{l}\text { FORTUNE BRANDS HOME } \\
\& \text { SECUR }\end{array}$ & FBHS & No & Yes & Yes \\
\hline $\begin{array}{l}\text { FREEPORT-MCMORAN } \\
\text { INC }\end{array}$ & FCX & No & Yes & No \\
\hline FEDEX CORP & FDX & No & Yes & Yes \\
\hline FIRSTENERGY CORP & $\mathrm{FE}$ & No & Yes & No \\
\hline F5 NETWORKS INC & FFIV & No & Yes & Yes \\
\hline $\begin{array}{l}\text { FIDELITY NATIONAL INFO } \\
\text { SVCS }\end{array}$ & FIS & No & Yes & Yes \\
\hline FISERV INC & FISV & No & Yes & Yes \\
\hline FIFTH THIRD BANCORP & FITB & No & Yes & Yes \\
\hline FOOT LOCKER INC & FL & No & Yes & No \\
\hline FLIR SYSTEMS INC & FLIR & No & Yes & Yes \\
\hline FLUOR CORP & FLR & No & Yes & No \\
\hline FLOWSERVE CORP & FLS & No & Yes & Yes \\
\hline
\end{tabular}




\begin{tabular}{|c|c|c|c|c|}
\hline $\begin{array}{l}\text { FLEETCOR TECHNOLO- } \\
\text { GIES INC }\end{array}$ & FLT & No & Yes & Yes \\
\hline FMC CORP & FMC & No & Yes & No \\
\hline FOX CORP & FOXA & No & Yes & No \\
\hline FIRST REPUBLIC BANK & FRC & No & Yes & No \\
\hline $\begin{array}{l}\text { FEDERAL REALTY INVEST- } \\
\text { MENT TR }\end{array}$ & FRT & No & Yes & No \\
\hline TECHNIPFMC PLC & FTI & No & Yes & No \\
\hline FORTINET INC & FTNT & No & Yes & No \\
\hline FORTIVE CORP & FTV & No & Yes & No \\
\hline $\begin{array}{l}\text { GENERAL DYNAMICS } \\
\text { CORP }\end{array}$ & GD & No & Yes & Yes \\
\hline GENERAL ELECTRIC CO & GE & No & Yes & Yes \\
\hline GILEAD SCIENCES INC & GILD & No & Yes & Yes \\
\hline GENERAL MILLS INC & GIS & No & Yes & No \\
\hline CORNING INC & GLW & No & Yes & Yes \\
\hline GENERAL MOTORS CO & GM & No & Yes & No \\
\hline ALPHABET INC & GOOGL & No & Yes & No \\
\hline GENUINE PARTS CO & GPC & No & Yes & Yes \\
\hline GLOBAL PAYMENTS INC & GPN & No & Yes & No \\
\hline GAP INC & GPS & No & Yes & Yes \\
\hline GARMIN LTD & GRMN & No & Yes & Yes \\
\hline
\end{tabular}




\begin{tabular}{|c|c|c|c|c|}
\hline $\begin{array}{l}\text { GOLDMAN SACHS GROUP } \\
\text { INC }\end{array}$ & GS & Yes & Yes & Yes \\
\hline GRAINGER (W W) INC & GWW & No & Yes & Yes \\
\hline HALLIBURTON CO & HAL & No & Yes & No \\
\hline HASBRO INC & HAS & No & Yes & Yes \\
\hline $\begin{array}{l}\text { HUNTINGTON BANC- } \\
\text { SHARES }\end{array}$ & HBAN & No & Yes & No \\
\hline HANESBRANDS INC & HBI & No & Yes & Yes \\
\hline HCA HEALTHCARE INC & HCA & No & Yes & No \\
\hline HCP INC & $\mathrm{HCP}$ & No & Yes & No \\
\hline HOME DEPOT INC & HD & Yes & Yes & Yes \\
\hline HESS CORP & HES & No & Yes & No \\
\hline HOLLYFRONTIER CORP & HFC & No & Yes & No \\
\hline $\begin{array}{l}\text { HARTFORD FINANCIAL } \\
\text { SERVICES }\end{array}$ & HIG & No & Yes & Yes \\
\hline $\begin{array}{l}\text { HUNTINGTON INGALLS } \\
\text { IND INC }\end{array}$ & HII & No & Yes & Yes \\
\hline $\begin{array}{ll}\text { HILTON } & \text { WORLDWIDE } \\
\text { HOLDINGS } & \end{array}$ & HLT & No & Yes & No \\
\hline HARLEY-DAVIDSON INC & HOG & No & Yes & Yes \\
\hline HOLOGIC INC & HOLX & No & Yes & Yes \\
\hline $\begin{array}{l}\text { HONEYWELL INTERNA- } \\
\text { TIONAL INC }\end{array}$ & $\mathrm{HON}$ & No & Yes & Yes \\
\hline
\end{tabular}




\begin{tabular}{|c|c|c|c|c|}
\hline HELMERICH \& PAYNE & $\mathrm{HP}$ & No & Yes & No \\
\hline $\begin{array}{l}\text { HEWLETT PACKARD EN- } \\
\text { TERPRISE }\end{array}$ & HPE & No & Yes & No \\
\hline HP INC & HPQ & No & Yes & No \\
\hline BLOCK H \& R INC & HRB & No & Yes & No \\
\hline HORMEL FOODS CORP & HRL & No & Yes & No \\
\hline HARRIS CORP & HRS & No & Yes & No \\
\hline HENRY SCHEIN INC & HSIC & No & Yes & Yes \\
\hline $\begin{array}{l}\text { HOST HOTELS \& RESORTS } \\
\text { INC }\end{array}$ & HST & No & Yes & No \\
\hline HERSHEY CO & HSY & No & Yes & No \\
\hline HUMANA INC & HUM & No & Yes & Yes \\
\hline $\begin{array}{l}\text { INTL BUSINESS MACHINES } \\
\text { CORP }\end{array}$ & IBM & Yes & Yes & Yes \\
\hline $\begin{array}{l}\text { INTERCONTINENTAL EX- } \\
\text { CHANGE }\end{array}$ & ICE & No & Yes & Yes \\
\hline IDEXX LABS INC & IDXX & No & Yes & No \\
\hline $\begin{array}{l}\text { INTL FLAVORS \& FRA- } \\
\text { GRANCES }\end{array}$ & IFF & No & Yes & No \\
\hline ILLUMINA INC & ILMN & No & Yes & Yes \\
\hline INCYTE CORP & INCY & No & Yes & No \\
\hline IHS MARKIT LTD & INFO & No & Yes & Yes \\
\hline INTEL CORP & INTC & Yes & Yes & Yes \\
\hline
\end{tabular}




\begin{tabular}{|c|c|c|c|c|}
\hline INTUIT INC & INTU & No & Yes & Yes \\
\hline INTL PAPER CO & IP & No & Yes & Yes \\
\hline $\begin{array}{l}\text { INTERPUBLIC GROUP OF } \\
\text { COS }\end{array}$ & IPG & No & Yes & Yes \\
\hline IPG PHOTONICS CORP & IPGP & No & Yes & Yes \\
\hline IQVIA HOLDINGS INC & IQV & No & Yes & No \\
\hline INGERSOLL-RAND PLC & IR & No & Yes & No \\
\hline IRON MOUNTAIN INC & IRM & No & Yes & No \\
\hline INTUITIVE SURGICAL INC & ISRG & No & Yes & No \\
\hline GARTNER INC & IT & No & Yes & No \\
\hline ILLINOIS TOOL WORKS & ITW & No & Yes & Yes \\
\hline INVESCO LTD & IVZ & No & Yes & No \\
\hline $\begin{array}{l}\text { HUNT (JB) TRANSPRT } \\
\text { SVCS INC }\end{array}$ & JBHT & No & Yes & Yes \\
\hline $\begin{array}{l}\text { JOHNSON CONTROLS } \\
\text { INTL PLC }\end{array}$ & JCI & No & Yes & No \\
\hline $\begin{array}{l}\text { JACOBS ENGINEERING } \\
\text { GROUP INC }\end{array}$ & JEC & No & Yes & No \\
\hline $\begin{array}{l}\text { JEFFERIES FINANCIAL GRP } \\
\text { INC }\end{array}$ & JEF & No & Yes & No \\
\hline $\begin{array}{l}\text { HENRY (JACK) \& ASSO- } \\
\text { CIATES }\end{array}$ & JKHY & No & Yes & No \\
\hline JOHNSON \& JOHNSON & JNJ & Yes & Yes & No \\
\hline
\end{tabular}




\begin{tabular}{|c|c|c|c|c|}
\hline JUNIPER NETWORKS INC & JNPR & No & Yes & Yes \\
\hline JPMORGAN CHASE \& CO & JPM & Yes & Yes & Yes \\
\hline NORDSTROM INC & JWN & No & Yes & Yes \\
\hline KELLOGG CO & $\mathrm{K}$ & No & Yes & Yes \\
\hline KEYCORP & KEY & No & Yes & Yes \\
\hline $\begin{array}{l}\text { KEYSIGHT TECHNOLO- } \\
\text { GIES INC }\end{array}$ & KEYS & No & Yes & Yes \\
\hline KRAFT HEINZ CO & $\mathrm{KHC}$ & No & Yes & No \\
\hline KIMCO REALTY CORP & KIM & No & Yes & No \\
\hline KLA-TENCOR CORP & KLAC & No & Yes & Yes \\
\hline KIMBERLY-CLARK CORP & $\mathrm{KMB}$ & No & Yes & No \\
\hline KINDER MORGAN INC & KMI & No & Yes & No \\
\hline CARMAX INC & KMX & No & Yes & No \\
\hline COCA-COLA CO & $\mathrm{KO}$ & Yes & Yes & No \\
\hline KROGER CO & $\mathrm{KR}$ & No & Yes & No \\
\hline KOHL'S CORP & KSS & No & Yes & Yes \\
\hline KANSAS CITY SOUTHERN & KSU & No & Yes & Yes \\
\hline LOEWS CORP & $\mathrm{L}$ & No & Yes & Yes \\
\hline L BRANDS INC & LB & No & Yes & No \\
\hline LEGGETT \& PLATT INC & LEG & No & Yes & Yes \\
\hline LENNAR CORP & LEN & No & Yes & No \\
\hline
\end{tabular}




\begin{tabular}{|c|c|c|c|c|}
\hline $\begin{array}{l}\text { LABORATORY CP OF } \\
\text { AMER HLDGS }\end{array}$ & $\mathrm{LH}$ & No & Yes & Yes \\
\hline LINDE PLC & LIN & No & Yes & Yes \\
\hline LKQ CORP & LKQ & No & Yes & Yes \\
\hline L3 TECHNOLOGIES INC & LLL & No & Yes & Yes \\
\hline LILLY (ELI) \& CO & LLY & No & Yes & Yes \\
\hline LOCKHEED MARTIN CORP & LMT & No & Yes & No \\
\hline $\begin{array}{l}\text { LINCOLN NATIONAL } \\
\text { CORP }\end{array}$ & LNC & No & Yes & Yes \\
\hline ALLIANT ENERGY CORP & LNT & No & Yes & Yes \\
\hline LOWE'S COMPANIES INC & LOW & No & Yes & Yes \\
\hline LAM RESEARCH CORP & LRCX & No & Yes & Yes \\
\hline SOUTHWEST AIRLINES & LUV & No & Yes & Yes \\
\hline $\begin{array}{l}\text { LAMB WESTON HOLD- } \\
\text { INGS INC }\end{array}$ & LW & No & Yes & No \\
\hline $\begin{array}{l}\text { LYONDELLBASELL INDUS- } \\
\text { TRIES NV }\end{array}$ & LYB & No & Yes & Yes \\
\hline MACY'S INC & M & No & Yes & No \\
\hline MASTERCARD INC & MA & No & Yes & Yes \\
\hline $\begin{array}{l}\text { MID-AMERICA APT CMN- } \\
\text { TYS INC }\end{array}$ & MAA & No & Yes & No \\
\hline MACERICH CO & MAC & No & Yes & No \\
\hline MARRIOTT INTL INC & MAR & No & Yes & No \\
\hline
\end{tabular}




\begin{tabular}{|c|c|c|c|c|}
\hline MASCO CORP & MAS & No & Yes & No \\
\hline MATTEL INC & MAT & No & Yes & No \\
\hline MCDONALD'S CORP & MCD & Yes & Yes & No \\
\hline $\begin{array}{l}\text { MICROCHIP TECHNOL- } \\
\text { OGY INC }\end{array}$ & MCHP & No & Yes & Yes \\
\hline MCKESSON CORP & MCK & No & Yes & Yes \\
\hline MOODY'S CORP & $\mathrm{MCO}$ & No & Yes & No \\
\hline $\begin{array}{l}\text { MONDELEZ INTERNA- } \\
\text { TIONAL INC }\end{array}$ & MDLZ & No & Yes & Yes \\
\hline MEDTRONIC PLC & MDT & No & Yes & Yes \\
\hline METLIFE INC & MET & No & Yes & Yes \\
\hline $\begin{array}{l}\text { MGM RESORTS INTERNA- } \\
\text { TIONAL }\end{array}$ & MGM & No & Yes & Yes \\
\hline $\begin{array}{l}\text { MOHAWK INDUSTRIES } \\
\text { INC }\end{array}$ & MHK & No & Yes & Yes \\
\hline MCCORMICK \& CO INC & MKC & No & Yes & No \\
\hline $\begin{array}{l}\text { MARTIN MARIETTA MA- } \\
\text { TERIALS }\end{array}$ & MLM & No & Yes & Yes \\
\hline $\begin{array}{l}\text { MARSH \& MCLENNAN } \\
\text { COS }\end{array}$ & MMC & No & Yes & Yes \\
\hline $3 \mathrm{M} \mathrm{CO}$ & MMM & Yes & Yes & No \\
\hline $\begin{array}{l}\text { MONSTER BEVERAGE } \\
\text { CORP }\end{array}$ & MNST & No & Yes & No \\
\hline
\end{tabular}




\begin{tabular}{|c|c|c|c|c|}
\hline ALTRIA GROUP INC & $\mathrm{MO}$ & No & Yes & Yes \\
\hline MOSAIC CO & MOS & No & Yes & Yes \\
\hline $\begin{array}{l}\text { MARATHON PETROLEUM } \\
\text { CORP }\end{array}$ & MPC & No & Yes & Yes \\
\hline MERCK \& CO & MRK & Yes & Yes & Yes \\
\hline MARATHON OIL CORP & $\mathrm{MRO}$ & No & Yes & No \\
\hline MORGAN STANLEY & MS & No & Yes & Yes \\
\hline MSCI INC & MSCI & No & Yes & Yes \\
\hline MICROSOFT CORP & MSFT & Yes & Yes & Yes \\
\hline $\begin{array}{l}\text { MOTOROLA SOLUTIONS } \\
\text { INC }\end{array}$ & MSI & No & Yes & No \\
\hline M \& T BANK CORP & MTB & No & Yes & Yes \\
\hline $\begin{array}{l}\text { METTLER-TOLEDO INTL } \\
\text { INC }\end{array}$ & MTD & No & Yes & Yes \\
\hline $\begin{array}{l}\text { MICRON TECHNOLOGY } \\
\text { INC }\end{array}$ & MU & No & Yes & Yes \\
\hline $\begin{array}{ll}\text { MAXIM INTEGRATED } \\
\text { PRODUCTS }\end{array}$ & MXIM & No & Yes & No \\
\hline MYLAN NV & MYL & No & Yes & No \\
\hline NOBLE ENERGY INC & NBL & No & Yes & Yes \\
\hline $\begin{array}{l}\text { NORWEGIAN CRUISE LINE } \\
\text { HLDGS }\end{array}$ & NCLH & No & Yes & Yes \\
\hline NASDAQ INC & NDAQ & No & Yes & Yes \\
\hline
\end{tabular}




\begin{tabular}{|c|c|c|c|c|}
\hline NEXTERA ENERGY INC & NEE & No & Yes & No \\
\hline $\begin{array}{l}\text { NEWMONT GOLDCORP } \\
\text { CORP }\end{array}$ & NEM & No & Yes & No \\
\hline NETFLIX INC & NFLX & No & Yes & Yes \\
\hline NISOURCE INC & $\mathrm{NI}$ & No & Yes & Yes \\
\hline NIKE INC -CL B & NKE & Yes & Yes & Yes \\
\hline NEKTAR THERAPEUTICS & NKTR & No & Yes & No \\
\hline NIELSEN HOLDINGS PLC & NLSN & No & Yes & Yes \\
\hline $\begin{array}{l}\text { NORTHROP GRUMMAN } \\
\text { CORP }\end{array}$ & NOC & No & Yes & No \\
\hline $\begin{array}{l}\text { NATIONAL OILWELL } \\
\text { VARCO INC }\end{array}$ & NOV & No & Yes & Yes \\
\hline NRG ENERGY INC & NRG & No & Yes & No \\
\hline $\begin{array}{l}\text { NORFOLK SOUTHERN } \\
\text { CORP }\end{array}$ & NSC & No & Yes & No \\
\hline NETAPP INC & NTAP & No & Yes & Yes \\
\hline NORTHERN TRUST CORP & NTRS & No & Yes & Yes \\
\hline NUCOR CORP & NUE & No & Yes & Yes \\
\hline NVIDIA CORP & NVDA & No & Yes & Yes \\
\hline NEWELL BRANDS INC & NWL & No & Yes & No \\
\hline NEWS CORP & NWSA & No & Yes & No \\
\hline REALTY INCOME CORP & $\mathrm{O}$ & No & Yes & No \\
\hline ONEOK INC & OKE & No & Yes & No \\
\hline
\end{tabular}




\begin{tabular}{|c|c|c|c|c|}
\hline OMNICOM GROUP & $\mathrm{OMC}$ & No & Yes & Yes \\
\hline ORACLE CORP & ORCL & No & Yes & Yes \\
\hline $\begin{array}{l}\text { O'REILLY AUTOMOTIVE } \\
\text { INC }\end{array}$ & ORLY & No & Yes & Yes \\
\hline $\begin{array}{l}\text { OCCIDENTAL } \\
\text { PETROLEUM CORP }\end{array}$ & OXY & No & Yes & No \\
\hline PAYCHEX INC & PAYX & No & Yes & Yes \\
\hline $\begin{array}{l}\text { PEOPLE'S UNITED FINL } \\
\text { INC }\end{array}$ & $\mathrm{PBCT}$ & No & Yes & No \\
\hline PACCAR INC & PCAR & No & Yes & Yes \\
\hline $\begin{array}{l}\text { PUBLIC SERVICE ENTRP } \\
\text { GRP INC }\end{array}$ & PEG & No & Yes & No \\
\hline PEPSICO INC & PEP & No & Yes & No \\
\hline PFIZER INC & PFE & Yes & Yes & No \\
\hline $\begin{array}{l}\text { PRINCIPAL FINANCIAL } \\
\text { GRP INC }\end{array}$ & PFG & No & Yes & Yes \\
\hline PROCTER \& GAMBLE CO & PG & Yes & Yes & No \\
\hline PROGRESSIVE CORP-OHIO & PGR & No & Yes & Yes \\
\hline PARKER-HANNIFIN CORP & $\mathrm{PH}$ & No & Yes & No \\
\hline PULTEGROUP INC & PHM & No & Yes & Yes \\
\hline $\begin{array}{l}\text { PACKAGING CORP OF } \\
\text { AMERICA }\end{array}$ & PKG & No & Yes & Yes \\
\hline PERKINELMER INC & PKI & No & Yes & No \\
\hline
\end{tabular}




\begin{tabular}{|c|c|c|c|c|}
\hline PROLOGIS INC & PLD & No & Yes & No \\
\hline $\begin{array}{l}\text { PHILIP MORRIS INTERNA- } \\
\text { TIONAL }\end{array}$ & PM & No & Yes & No \\
\hline $\begin{array}{l}\text { PNC FINANCIAL SVCS } \\
\text { GROUP INC }\end{array}$ & PNC & No & Yes & Yes \\
\hline PENTAIR PLC & PNR & No & Yes & Yes \\
\hline $\begin{array}{l}\text { PINNACLE WEST CAPITAL } \\
\text { CORP }\end{array}$ & PNW & No & Yes & Yes \\
\hline PPG INDUSTRIES INC & PPG & No & Yes & No \\
\hline PPL CORP & PPL & No & Yes & Yes \\
\hline PERRIGO CO PLC & PRGO & No & Yes & Yes \\
\hline $\begin{array}{l}\text { PRUDENTIAL FINANCIAL } \\
\text { INC }\end{array}$ & PRU & No & Yes & No \\
\hline PUBLIC STORAGE & PSA & No & Yes & No \\
\hline PHILLIPS 66 & PSX & No & Yes & Yes \\
\hline PVH CORP & $\mathrm{PVH}$ & No & Yes & Yes \\
\hline QUANTA SERVICES INC & PWR & No & Yes & No \\
\hline $\begin{array}{l}\text { PIONEER NATURAL RE- } \\
\text { SOURCES CO }\end{array}$ & PXD & No & Yes & Yes \\
\hline PAYPAL HOLDINGS INC & PYPL & No & Yes & Yes \\
\hline QUALCOMM INC & QCOM & No & Yes & Yes \\
\hline QORVO INC & QRVO & No & Yes & Yes \\
\hline
\end{tabular}




\begin{tabular}{|c|c|c|c|c|}
\hline $\begin{array}{l}\text { ROYAL CARIBBEAN } \\
\text { CRUISES LTD }\end{array}$ & $\mathrm{RCL}$ & No & Yes & Yes \\
\hline EVEREST RE GROUP LTD & $\mathrm{RE}$ & No & Yes & Yes \\
\hline REGENCY CENTERS CORP & REG & No & Yes & No \\
\hline $\begin{array}{l}\text { REGENERON PHARMA- } \\
\text { CEUTICALS }\end{array}$ & REGN & No & Yes & No \\
\hline $\begin{array}{l}\text { REGIONS FINANCIAL } \\
\text { CORP }\end{array}$ & $\mathrm{RF}$ & No & Yes & Yes \\
\hline ROBERT HALF INTL INC & RHI & No & Yes & No \\
\hline RED HAT INC & RHT & No & Yes & Yes \\
\hline $\begin{array}{l}\text { RAYMOND JAMES FINAN- } \\
\text { CIAL CORP }\end{array}$ & RJF & No & Yes & No \\
\hline RALPH LAUREN CORP & $\mathrm{RL}$ & No & Yes & Yes \\
\hline RESMED INC & RMD & No & Yes & Yes \\
\hline $\begin{array}{l}\text { ROCKWELL AUTOMA- } \\
\text { TION }\end{array}$ & $\mathrm{ROK}$ & No & Yes & No \\
\hline ROLLINS INC & ROL & No & Yes & No \\
\hline $\begin{array}{l}\text { ROPER TECHNOLOGIES } \\
\text { INC }\end{array}$ & $\mathrm{ROP}$ & No & Yes & Yes \\
\hline ROSS STORES INC & ROST & No & Yes & Yes \\
\hline REPUBLIC SERVICES INC & RSG & No & Yes & Yes \\
\hline RAYTHEON CO & RTN & No & Yes & No \\
\hline
\end{tabular}




\begin{tabular}{|c|c|c|c|c|}
\hline $\begin{array}{l}\text { SBA COMMUNICATIONS } \\
\text { CORP }\end{array}$ & SBAC & No & Yes & No \\
\hline STARBUCKS CORP & SBUX & No & Yes & Yes \\
\hline SCHWAB (CHARLES) CORP & SCHW & No & Yes & Yes \\
\hline SEALED AIR CORP & SEE & No & Yes & No \\
\hline SHERWIN-WILLIAMS CO & SHW & No & Yes & Yes \\
\hline SVB FINANCIAL GROUP & SIVB & No & Yes & No \\
\hline SMUCKER (JM) CO & SJM & No & Yes & No \\
\hline SCHLUMBERGER LTD & SLB & No & Yes & No \\
\hline SL GREEN REALTY CORP & SLG & No & Yes & Yes \\
\hline SNAP-ON INC & SNA & No & Yes & Yes \\
\hline SYNOPSYS INC & SNPS & No & Yes & Yes \\
\hline SOUTHERN CO & $\mathrm{SO}$ & No & Yes & No \\
\hline $\begin{array}{l}\text { SIMON PROPERTY GROUP } \\
\text { INC }\end{array}$ & SPG & No & Yes & No \\
\hline S\&P GLOBAL INC & SPGI & No & Yes & Yes \\
\hline SEMPRA ENERGY & SRE & No & Yes & No \\
\hline SUNTRUST BANKS INC & STI & No & Yes & Yes \\
\hline STATE STREET CORP & STT & No & Yes & Yes \\
\hline $\begin{array}{l}\text { SEAGATE TECHNOLOGY } \\
\text { PLC }\end{array}$ & STX & No & Yes & Yes \\
\hline CONSTELLATION BRANDS & STZ & No & Yes & No \\
\hline
\end{tabular}




\begin{tabular}{|c|c|c|c|c|}
\hline $\begin{array}{lll}\text { STANLEY BLACK } & \& \\
\text { DECKER INC } & \end{array}$ & SWK & No & Yes & Yes \\
\hline $\begin{array}{l}\text { SKYWORKS SOLUTIONS } \\
\text { INC }\end{array}$ & SWKS & No & Yes & No \\
\hline SYNCHRONY FINANCIAL & SYF & No & Yes & Yes \\
\hline STRYKER CORP & SYK & No & Yes & Yes \\
\hline SYMANTEC CORP & SYMC & No & Yes & Yes \\
\hline SYSCO CORP & SYY & No & Yes & Yes \\
\hline AT\&T INC & $\mathrm{T}$ & No & Yes & Yes \\
\hline $\begin{array}{l}\text { MOLSON COORS BREW- } \\
\text { ING CO }\end{array}$ & TAP & No & Yes & No \\
\hline TRANSDIGM GROUP INC & TDG & No & Yes & No \\
\hline TE CONNECTIVITY LTD & TEL & No & Yes & Yes \\
\hline $\begin{array}{l}\text { TWENTY-FIRST CENTURY } \\
\text { FOX INC }\end{array}$ & TFCFA & No & Yes & No \\
\hline TELEFLEX INC & TFX & No & Yes & Yes \\
\hline TARGET CORP & TGT & No & Yes & No \\
\hline TIFFANY \& CO & TIF & No & Yes & Yes \\
\hline TJX COMPANIES INC & TJX & No & Yes & No \\
\hline TORCHMARK CORP & TMK & No & Yes & Yes \\
\hline $\begin{array}{l}\text { THERMO FISHER SCIEN- } \\
\text { TIFIC INC }\end{array}$ & TMO & No & Yes & Yes \\
\hline TAPESTRY INC & TPR & No & Yes & Yes \\
\hline
\end{tabular}




\begin{tabular}{|c|c|c|c|c|}
\hline TRIPADVISOR INC & TRIP & No & Yes & Yes \\
\hline PRICE (T. ROWE) GROUP & TROW & No & Yes & Yes \\
\hline TRAVELERS COS INC & TRV & Yes & Yes & No \\
\hline TRACTOR SUPPLY CO & TSCO & No & Yes & Yes \\
\hline TYSON FOODS INC -CL A & TSN & No & Yes & Yes \\
\hline $\begin{array}{l}\text { TOTAL SYSTEM SERVICES } \\
\text { INC }\end{array}$ & TSS & No & Yes & Yes \\
\hline $\begin{array}{l}\text { TAKE-TWO INTERACTIVE } \\
\text { SFTWR }\end{array}$ & TTWO & No & Yes & Yes \\
\hline TWITTER INC & TWTR & No & Yes & Yes \\
\hline TEXAS INSTRUMENTS INC & $\mathrm{TXN}$ & No & Yes & No \\
\hline TEXTRON INC & TXT & No & Yes & Yes \\
\hline UNDER ARMOUR INC & UAA & No & Yes & No \\
\hline $\begin{array}{l}\text { UNITED CONTINENTAL } \\
\text { HLDGS INC }\end{array}$ & UAL & No & Yes & No \\
\hline UDR INC & UDR & No & Yes & No \\
\hline $\begin{array}{l}\text { UNIVERSAL HEALTH SVCS } \\
\text { INC }\end{array}$ & UHS & No & Yes & Yes \\
\hline ULTA BEAUTY INC & ULTA & No & Yes & Yes \\
\hline $\begin{array}{l}\text { UNITEDHEALTH GROUP } \\
\text { INC }\end{array}$ & $\mathrm{UNH}$ & Yes & Yes & Yes \\
\hline UNUM GROUP & UNM & No & Yes & No \\
\hline UNION PACIFIC CORP & UNP & No & Yes & Yes \\
\hline
\end{tabular}




\begin{tabular}{|c|c|c|c|c|}
\hline $\begin{array}{l}\text { UNITED PARCEL SERVICE } \\
\text { INC }\end{array}$ & UPS & No & Yes & Yes \\
\hline UNITED RENTALS INC & URI & No & Yes & No \\
\hline U S BANCORP & USB & No & Yes & Yes \\
\hline $\begin{array}{l}\text { UNITED TECHNOLOGIES } \\
\text { CORP }\end{array}$ & UTX & Yes & Yes & Yes \\
\hline VISA INC & $\mathrm{V}$ & Yes & Yes & Yes \\
\hline $\begin{array}{l}\text { VARIAN MEDICAL SYS- } \\
\text { TEMS INC }\end{array}$ & VAR & No & Yes & No \\
\hline VF CORP & VFC & No & Yes & Yes \\
\hline VIACOM INC & VIAB & No & Yes & No \\
\hline VALERO ENERGY CORP & VLO & No & Yes & Yes \\
\hline VULCAN MATERIALS CO & VMC & No & Yes & Yes \\
\hline VORNADO REALTY TRUST & VNO & No & Yes & No \\
\hline VERISK ANALYTICS INC & VRSK & No & Yes & No \\
\hline VERISIGN INC & VRSN & No & Yes & No \\
\hline $\begin{array}{l}\text { VERTEX PHARMACEUTI- } \\
\text { CALS INC }\end{array}$ & VRTX & No & Yes & Yes \\
\hline VENTAS INC & VTR & No & Yes & No \\
\hline $\begin{array}{l}\text { VERIZON COMMUNICA- } \\
\text { TIONS INC }\end{array}$ & $\mathrm{VZ}$ & Yes & Yes & Yes \\
\hline WABTEC CORP & WAB & No & Yes & No \\
\hline WATERS CORP & WAT & No & Yes & Yes \\
\hline
\end{tabular}




\begin{tabular}{|c|c|c|c|c|}
\hline $\begin{array}{l}\text { WALGREENS BOOTS AL- } \\
\text { LIANCE INC }\end{array}$ & WBA & Yes & Yes & No \\
\hline $\begin{array}{l}\text { WELLCARE HEALTH } \\
\text { PLANS INC }\end{array}$ & WCG & No & Yes & Yes \\
\hline WESTERN DIGITAL CORP & WDC & No & Yes & No \\
\hline WEC ENERGY GROUP INC & WEC & No & Yes & No \\
\hline WELLTOWER INC & WELL & No & Yes & No \\
\hline WELLS FARGO \& CO & WFC & No & Yes & No \\
\hline WHIRLPOOL CORP & WHR & No & Yes & No \\
\hline $\begin{array}{l}\text { WILLIS TOWERS WATSON } \\
\text { PLC }\end{array}$ & WLTW & No & Yes & Yes \\
\hline $\begin{array}{l}\text { WASTE MANAGEMENT } \\
\text { INC }\end{array}$ & WM & No & Yes & Yes \\
\hline WILLIAMS COS INC & WMB & No & Yes & No \\
\hline WALMART INC & WMT & Yes & Yes & Yes \\
\hline WESTROCK CO & WRK & No & Yes & Yes \\
\hline WESTERN UNION CO & WU & No & Yes & No \\
\hline WEYERHAEUSER CO & WY & No & Yes & No \\
\hline WYNN RESORTS LTD & WYNN & No & Yes & No \\
\hline CIMAREX ENERGY CO & XEC & No & Yes & Yes \\
\hline XCEL ENERGY INC & XEL & No & Yes & Yes \\
\hline XILINX INC & XLNX & No & Yes & Yes \\
\hline EXXON MOBIL CORP & $\mathrm{XOM}$ & Yes & Yes & Yes \\
\hline
\end{tabular}




\begin{tabular}{|l|l|l|l|l|}
\hline DENTSPLY SIRONA INC & XRAY & No & Yes & Yes \\
\hline XEROX CORP & XRX & No & Yes & No \\
\hline XYLEM INC & XYL & No & Yes & Yes \\
\hline YUM BRANDS INC & YUM & No & Yes & No \\
\hline ZIMMER BIOMET HOLD- & ZBH & No & Yes & Yes \\
\hline INGS INC & & & & \\
\hline ZIONS BANCORPORATION & ZION & No & Yes & Yes \\
\hline NA & ZTS & No & Yes & Yes \\
\hline ZOETIS INC & ZIO & & \\
\hline
\end{tabular}


CHAPTER 4

\section{THE ROLE OF 'OTHER INFORMATION’ $\nu_{T}$ AS A VALUE-RELEVANT MEASURE IN TRACKING AND PREDICTING STOCK RETURNS: A FAVAR APPROACH}

\subsection{Introduction}

Ohlson (1995) has become seminal work within the accounting and finance literature. The paper comprises of two main parts. The residual income valuation model (RIM) and the residual income information dynamics. Ohlson's modelling of the latter is the primary contribution in the paper. The key feature of the information dynamics is that expected next period $t+1$ abnormal earnings ${ }^{1}$ contains information known at time $t$ that is not present in current abnormal earnings. Ota (2002) states that the linear information dynamics attempt to characterize the mechanism of abnormal earnings and links current information to future abnormal earnings. This enables the formulation of a valuation model of a stock.

Ohlson (1995) models this information about future abnormal earnings not reflected in current abnormal earnings as a latent variable denoted as 'other information' $\nu_{t}$. Specifically, $\nu_{t}$ summarizes unobserved value-relevant information about events and their effect on future profitability that is captured in a company's current stock price, but not yet reflected in a company's current financial statements.

Dechow et al. (1999) (henceforth DHS) are the first to include the residual income information dynamics explicitly in their study. They use Wall Street consensus analysts' earnings forecasts to proxy 'other information' $\nu_{t}$. They argue that Wall Street analysts have better sets of information to predict future abnormal earnings and, in turn, price stocks effectively, relative to retail investors. However, there are many issues with the use of analyst forecasts of earnings as a proxy for 'other information' $\nu_{t}$ (see Awwal and Bidarkota (2019) for related discussion).

Several papers have attempted to model or estimate 'other information' $\nu_{t}$ for valuation using various accounting variables (e.g. see Hand and Landsman (1998, 2005), Barth et al. (1999), Myers

\footnotetext{
${ }^{1}$ We use 'abnormal earnings' and 'residual income' interchangeably throughout the paper.
} 
(1999), and, most recently, Yamaguchi (2018) for details). Though, none of which has achieved as much empirical success as the use of Wall Street analysts' earnings forecasts.

Another strand of research involves attempts to find value-relevant measures for predicting future stock returns with varying degrees of success. For example, Lettau and Ludvigson (2001) use the aggregate consumption-wealth ratio for predicting returns. Lee et. al (1999) use intrinsic value measures based on the residual income model to track current prices and predict future market returns. However, Goyal and Welch (2007) argue that many popular predictor variables used in the extant literature have poor in- and out-of-sample predictive ability and consistently underperform against the historical mean model.

The aim of this paper is to estimate Ohlson's 'other information' $\nu_{t}$ and to learn about its time-series properties and its ability to track and predict stock returns. We do so by utilizing the theoretical framework of the RIM in a factor augmented vector autoregressive (FAVAR) system developed by Bernanke et al. (2005) (henceforth BBE). The FAVAR allows us to examine the linkages of stock market value, residual income, and low-dimensional unobserved factors that are broadly summarized by a rich set of data. These together are common factors to the system. In the context of VAR models, the FAVAR's ability to estimate a low dimension of unobserved factors that reflect informational content of big data makes it a suitable model to accomplish our efforts. ${ }^{2}$ In total, we consider 78 informational variables across financial market, accounting, investor and consumer sentiment, and macroeconomic data to extract the unobserved factors. We use a two-step principal components approach, as in BBE, to extract the latent factors.

The FAVAR model is widely used in various economic applications (see, for example, Ludvigson and Ng (2009), Koop and Korobilis (2010), Bai et al. (2016), Stock and Watson (2016), Paccagnini (2018), and Fiorelli and Meliciani (2019), amongst many others). However, to the best of our knowledge, no existing papers have employed FAVAR or any similar application of factor analysis to explore 'other information' within the RIM framework.

\footnotetext{
${ }^{2} \mathrm{~A}$ standard problem in multi-variate VAR models is the depletion of degrees of freedom, as more variables are added. The low dimension of the unobserved factors mitigates this problem.
} 
In this paper, we estimate 'other information' $\nu_{t}$ using a two-step principal components approach under the FAVAR framework to assess to evaluate its success as a value-relevant measure. A viable measure would satisfy tracking ability of current stock returns and forecasting ability of future returns. Given that the FAVAR system makes use of the unobserved factors, we also analyze the sources of information that drive such unobserved factors. This allows us to pinpoint which type of information is value-relevant.

We organize the paper as follows. In section 2, we provide a framework of the RIM by describing the information dynamics and the implied valuation function. We also discuss the estimation of the 'other information' $\nu_{t}$, proxied by analysts' forecasts on earnings, as in DHS. In section 3, we set a formal structure for the FAVAR model in the context of Ohlson's RIM framework. We discuss how we use estimation of the FAVAR to measure $\nu_{t}$. We then detail the two-step principal components estimation approach to estimate the FAVAR itself and an identification strategy rationalizing the analysis. In section 4, we discuss the data and report empirical results. We summarize our findings in the concluding section.

\subsection{Residual Income Model (RIM)}

In this section, we lay the linear information dynamics framework and implied pricing function in Ohlson (1995)'s RIM. We then describe the estimation of the 'other information' $\nu_{t}$ proxied by consensus analysts' earnings forecasts.

\subsubsection{Residual Income Information Dynamics and Implied Valu- ation Function}

Ohlson (1995) defines abnormal earnings as the difference between earnings at time $t, x_{t}$, and the equity capital charge, $r \cdot b_{t-1} . r$ is the discount rate and $b_{t-1}$ is the book value of equity at time $t-1$ for a firm. 
He assumes that abnormal earnings satisfy the following modified autoregressive process:

$$
\begin{gathered}
x_{t}^{a}=\omega x_{t-1}^{a}+v_{t-1}+\varepsilon_{t}, \quad \varepsilon_{t} \sim i i d N\left(0, \sigma_{\varepsilon}^{2}\right) \\
v_{t}=\gamma v_{t-1}+\eta_{t}, \quad \eta_{t} \sim \text { iid } N\left(0, \sigma_{\eta}^{2}\right),
\end{gathered}
$$

where $\nu_{t}$ is 'other information' about next period $t+1$ abnormal earnings $x_{t+1}^{a}$ not conveyed by current abnormal earnings $x_{t}^{a}, \varepsilon_{t}$ and $\eta_{t}$ are unpredictable, mean zero disturbance terms, and $\omega$ and $\gamma$ are fixed persistence parameters, assumed non-negative and less than one.

Ohlson (1995) also derives the following implied valuation function:

$$
p_{t}-b_{t}=\alpha_{1} x_{t}^{a}+\alpha_{2} v_{t}
$$

where $\alpha_{1}=\omega /(1+r-\omega)$ and $\alpha_{2}=(1+r) /[(1+r-\omega)(1+r-\gamma)]$. Eq. (2) implies that the difference between the market value of equity $p_{t}$ and book value of equity $b_{t}$ at time $t$ for a firm reflects the discounted value of current abnormal earnings and 'other information' about future profitability. The right hand side of Eq. (2) is denoted as 'goodwill'.

\subsection{2 'Other information' $\nu_{t}$}

The main theme of this paper surrounds this latent 'other information' $\nu_{t}$ in Ohlson (1995). He postulates that a firm's stock price reflects information about its next period $t+1$ profitability that is not readily reflected in current profits. Furthermore, he formulates the 'other information' variable, $\nu_{t}$, as the difference between the conditional expectation of abnormal earnings for next period $t+1$ based on all available information at time $t$ and that based on current period $t$ abnormal earnings alone:

$$
v_{t}=\mathrm{E}_{t}\left[x_{t+1}^{\mathrm{a}}\right]-\omega x_{t}^{\mathrm{a}}
$$

${ }^{3}$ We use 'goodwill' to represent stock market value throughout the paper. Additionally, to adhere to Equation (2) in the RIM, we reference $p_{t}-b_{t}$ as 'goodwill' throughout the paper as well. 
The conditional expectation of next period $t+1$ abnormal earnings, denoted as $f_{t}^{a}$ below, is equal to the conditional expectation of next period $t+1$ earnings at time $t$ less the product of book value at time $t$ and the discount rate. DHS measure the conditional expectation of next period $t+1$ earnings at time $t$ using the consensus analyst forecast of next period $t+1$ earnings, denoted as $f_{t}$, in which case

$$
\mathrm{E}_{t}\left[x_{t+1}^{\mathrm{a}}\right]=f_{t}^{\mathrm{a}}=f_{t}-r \cdot b_{t} .
$$

Therefore, from Eqs. (3) and (4), 'other information', $\nu_{t}$ can be retrieved as

$$
v_{t}=f_{t}^{a}-\omega x_{t}^{\mathrm{a}}
$$

The values for the three parameters, namely $\omega, \gamma$, and $r$ in Eqs. (1) and (2) are pinned down as follows. The authors use the average historical return on equities to measure the discount rate, $r$, which they approximated to be $12 \%$. We employ time-varying discount rates, proxied by a 90 -day Treasury Bill when constructing abnormal earnings used in the empirical analysis later in the paper. We do this to account for time-varying risk. They estimate the persistence parameters, $\omega$ and $\gamma$, which they postulate as common across firms, by using unconditional pooled regressions (see DHS for details).

\subsection{FAVAR Application}

In this section, we first set a formal structure for the FAVAR model within Ohlson's RIM framework. We later provide the two-step principal components estimation approach of the FAVAR and impose restrictions on the system and data sources.

\subsubsection{Framework}

Let $Y_{t}$ be a $M \times 1$ vector of observable variables relevant to Ohlson's RI model and assumed to drive the dynamics of each other. $Y_{t}$ could contain return on equity (ROE) and $\mathrm{B} / \mathrm{P}$ ratio. 
The standard approach involves estimating a VAR using data for $Y_{t}$ alone.

Though, additional information, not fully captured by $Y_{t}$, may be relevant to modelling the dynamics of these series. Let $F_{t}$ be a $K \times 1$ vector of unobserved factors encompassing this additional information, where $\mathrm{K}$ is "small." These unobserved factors may capture fluctuations from an array of variables that are value-relevant. Such informational variables could include accounting, macroeconomic, consumer confidence, and financial market variables that may be intrinsically related to the observed variables in $Y_{t}$.

Assume that the joint dynamics of $\left(F_{t}^{\prime}, Y_{t}^{\prime}\right)$ are given by the following state equation:

$$
\left[\begin{array}{l}
F_{t} \\
Y_{t}
\end{array}\right]=\Phi(L)\left[\begin{array}{c}
F_{t-1} \\
Y_{t-1}
\end{array}\right]+v_{t}
$$

where $\Phi(L)$ is a conformable lag polynomial of finite order $d$, which may contain a priori restrictions as in the structural VAR literature. The error term $v_{t}$ has a zero mean and covariance matrix $Q$. Equation (6) is a VAR in $\left(F_{t}^{\prime}, Y_{t}^{\prime}\right)$. We refer to Equation (6) as FAVAR. This system can reduce to a standard VAR in $Y_{t}$ when the terms of $\Phi(L)$ that relate $Y_{t}$ to $F_{t-1}$ are all zero.

Equation (6) cannot be estimated directly because the factors $F_{t}$ are unobserved. However, as we interpret the factors as encapsulating informational content from a large set of economic variables, we may use data on a variety of economic time series to extrapolate the factors $F_{t}$. As such, let $X_{t}$ be a $N \times 1$ vector of informational time series. $N$ is "large" and may be greater than the number of time periods $T$. We assume $N$ to be strictly greater than the number of factors and observed variables in the FAVAR system such that $K+M<<N$. We also assume that the informational time series $X_{t}$ are related to the unobserved factors $F_{t}$ and the observed variables $Y_{t}$ by the following measurement equation:

$$
X_{t}=\Lambda^{f} F_{t}+\Lambda^{y} Y_{t}+e_{t},
$$


where $\Lambda^{\mathrm{f}}$ is a $N \times K$ matrix of factor loadings, $\Lambda^{\mathrm{y}}$ is $N \times M$, and the $N \times 1$ vector of error terms $e_{t}$ is mean zero, normally distributed, and has small cross-correlation. ${ }^{4}$ This representation nests models in which $X_{t}$ depends on lagged values of the factors $F_{t}$.

Equation (7) captures the idea that both $Y_{t}$ and $F_{t}$, which generally can be correlated, represent common forces that drive the dynamics of $X_{t}$. Conditional on $Y_{t}, X_{t}$ is therefore a vector of noisy measures of the underlying unobserved factors $F_{t}$. Without observed factor $Y_{t}$, Equation (7) reduces to a dynamic factor model (see Stock and Watson $(1998,2002)$ for further details).

\subsubsection{Estimation}

To estimate $\nu_{t}$ in Equation (5), we have to estimate the FAVAR specification to obtain fitted values of the ROE. The fitted values of ROE represent the conditional expectation of next-period $t+1 \mathrm{ROE}$ at time $t .^{5}$ For the estimation of the FAVAR model, Equations (6) and (7), we follow the two-step principal components approach as in BBE. As they note, “...provides a non-parametric way of uncovering the common space spanned by the factors of $X_{t} \ldots$. They denote this common space as $C\left(F_{t}, Y_{t}\right)$.

In the first step, the space spanned by the factors is estimated from the measurement equation in Eq. (7) using the first $K+M$ principal components of $X_{t}$, even in the presence of slight changes in the loading matrix $\Lambda$ (Paccagnini (2018)). They denote this estimated common space as $\hat{C}\left(F_{t}, Y_{t}\right)$.

Estimation of the first step does not make use of the fact that $Y_{t}$ is observed. Stock and Watson (2002) showed that the principal components consistently recover the space spanned by both $F_{t}$ and $Y_{t}$, when $N$ is large and the number of principal components $K+M$ used is at least as large as the true number of factors.

\footnotetext{
${ }^{4}$ The estimator can allow for some cross-correlation in $e_{t}$ that must vanish as $N$ goes to infinity.

${ }^{5}$ One can use simple algebraic manipulation to convert ROE to residual income.
} 
Bai and Ng (2002) and Lopes and West (2004) provide selection criteria to determine the optimal number of factors present in the $X_{t}$ data set. ${ }^{6}$

Since $\hat{C}\left(F_{t}, Y_{t}\right)$ corresponds to an arbitrary linear combination of its arguments, obtaining $\hat{F}_{t}$ involves determining the part of $\hat{C}\left(F_{t}, Y_{t}\right)$ that is not spanned by $Y_{t}$ (see BBE for details).

In the second step, the FAVAR in Equation (6) is estimated by regression methods, with $F_{t}$

replaced by $\hat{F}_{t} . Y_{t}$ is removed from the space covered by the principal components in the first step. Boivin et al. (2009) impose the constraint that $Y_{t}$ is one of the common components in the first step, guaranteeing that the estimated latent factors $\hat{F}_{t}$ recover the common dynamics, which are not captured by $Y_{t}$.

We use this two-step procedure for computational simplicity and execution, with few distributional assumptions and it allows for some cross-correlation in the idiosyncratic error term $e_{t}$ (see Stock and Watson (2002) for discussion).

\subsubsection{Identification Strategy}

At current form, Equations (6) and (7) of the FAVAR system are econometrically unidentified and therefore cannot be estimated directly. As such, we need to impose a set of restrictions on the system as in BBE and restrictions on the data sources. The first is a minimum set of normalization (orthogonality) restrictions on the measurement equation in Equation (7) that are needed to be able to estimate the model. The second is a restriction on the variables of the data to be on an aggregate or macro level.

First, we impose the orthogonality restriction in the principal components. In other words, we take $C^{\prime} C / T=I$, where $C^{\prime}=\left[C\left(F_{1}, Y_{1}\right), \ldots, C\left(F_{T}, Y_{T}\right)\right]$. This implies that $\hat{C}=\sqrt{T} \hat{Z}$, where $\hat{Z}$ are the eigenvectors corresponding to the $K$ largest eigenvalues of $X X^{\prime}$, sorted in descending order.

Second, though the Ohlson (1995) model is built in the context of an individual firm, we restrict the accounting and financial market data (i.e. equity, bond, and futures) used in the analysis

${ }^{6} \mathrm{We}$ do not make use of the selection criteria from either work, as they are beyond the scope of this paper. 
to an aggregate index level, namely, the S\&P 500. We presume that news or shocks affecting an entire stock index, such as the S\&P 500, rather than an individual stock, could plausibly have an impact on the overall economy and financial system.

\subsection{Empirical Assessment of the Model}

\subsubsection{Data Aggregation}

In this application, the aggregate data set $X_{t}$ composes of a balanced panel of 78 quarterly time series across financial market (FM), accounting, macroeconomic, and investor and consumer sentiment (ICS) data. We source FM data from CRSP, Robert Shiller's website, ${ }^{7}$ and Ken French's website. ${ }^{8}$. Accounting data for the S\&P 500 are sourced from COMPUSTAT. Macroeconomic data are sourced from the Federal Reserve Economic Data (FRED) - Federal Reserve Bank of St. Louis, ${ }^{9}$. Investor and consumer confidence data are sourced from the International Center for Finance at Yale School of Management. ${ }^{10}$

In $Y_{t}$, return on equity $(\mathrm{ROE})$ and $\mathrm{B} / \mathrm{P}$ ratio, constructed using the relevant accounting and financial market variables for the S\&P 500 are retrieved from CRSP and COMPUSTAT. S\&P 500 index return data are retrieved from CRSP. To construct 'other information' $\nu_{t}$ as described in Equation (5), we estimate two things. First, we calculate expected $t+1$ abnormal earnings as described in Equation (4). To do so, in addition to using the accounting data, we extract $t+1$ analyst earnings forecast data for the S\&P 500 from the I/B/E/S data file. Second, we estimate the persistence parameter, $\omega$, obtained from a first-order autoregression of the abnormal earnings time series, which equals 0.96 . For both abnormal earnings and expected $t+1$ abnormal earnings,

\footnotetext{
${ }^{7}$ Source: http://www.econ.yale.edu/ shiller/data.htm

${ }^{8}$ Source: http://mba.tuck.dartmouth.edu/pages/faculty/ken.french/data_library.html

${ }^{9}$ Source: https://fred.stlouisfed.org

${ }^{10}$ Source: https://som.yale.edu/faculty-research-centers/centers-initiatives/internationalcenter-for-finance/data/stock-market-confidence-indices/united-states-stock-marketconfidence-indices
} 
we use the 90-day Treasury Bill to proxy for the time-varying discount rate. ${ }^{11}$. Treasury Bill data are retrieved from CRSP as well.

The macroeconomic and FM data, including the S\&P 500 return data, retrieved span the period from 1993Q1 through 2018Q4. The accounting and forecast data retrieved span the same period, but in annual frequency. We use a piece-wise cubic spline interpolation in order to convert the accounting and forecast data into quarterly frequency. To ensure a balanced panel, we conduct the analysis beginning in the period of 1994Q1 to 2018Q4. ${ }^{12}$

All $X_{t}$ and $Y_{t}$ series are transformed appropriately to achieve stationarity. The description of the series in the aggregate data set and their corresponding transformation are provided in the appendix.

\subsubsection{Empirical Results}

In this section, we provide a set of empirical results to assess the viability, forecasting performance, and determinants of the estimated 'other information' $\nu_{t}$ under the FAVAR framework. The FAVAR model includes return on equity (ROE) and $\mathrm{B} / \mathrm{P}$ ratio, which are the only observed factors in $Y_{t}$, and two unobserved factors in $F_{t}$, which is estimated by the two-step principal components method. We have this specification for two reasons. First, we select ROE and B/P as $Y_{t}$ variables in order to adhere to Ohlson (1995), as the paper explicitly assumes that profitability and the accounting value of a firm's equity are relevant to contemporaneous market value and returns. Second, we include two unobserved factors to ensure we model all value-relevant information from big data. ${ }^{13}$

First, we evaluate the tracking ability and predictive power of 'other information' $\nu_{t}$ estimated under the FAVAR and compare it with two alternative measures to estimate $\nu_{t}$. The first

\footnotetext{
${ }^{11}$ We use several constant discount rates, ranging from $9 \%$ to $12 \%$, in the empirical analysis for robustness. We find no substantial differences in the results.

${ }^{12} \mathrm{We}$ lose degrees of freedom once we incorporate lags in the FAVAR system.

${ }^{13}$ We find that modelling either one, two, or three unobserved factors do not alter the results substantially.
} 
alternative measure to estimate $\nu_{t}$ is under a standard bi-variate VAR. This specification only has a $Y_{t}$ vector and includes return on equity and $\mathrm{B} / \mathrm{P}$ ratio, as in the FAVAR. The second alternative measure proxies $\nu_{t}$ with Wall Street analysts' earnings forecasts, as done in DHS. Accounting and finance literature together emphasize the significance of value-relevant measures that track current returns and predict future returns. A favorable estimate of 'other information' $\nu_{t}$ scaled by market value or book value results into a value-relevant ratio, $\frac{\nu_{t}}{p_{t}}$ or $\frac{\nu_{t}}{b_{t}}$, that has similar sample moments, a faster mean-reversion rate, and predicts future returns better. This suggests that the estimated value-relevant ratio has a lower degree of tracking error and mean-reverts quickly when it deviates away from the mean error.

Second, we implement several sets of univariate and multivariate forecasting regressions of quarterly S\&P 500 returns. We first run univariate regressions of one-quarter ahead returns on each of our FAVAR-derived value-relevant ratio estimates (henceforth, baseline predictor variables), as well as each traditional valuation ratio, including the $\mathrm{B} / \mathrm{P}$ ratio, $\mathrm{E} / \mathrm{P}$ ratio, $\mathrm{D} / \mathrm{P}$ ratio, and ROE (henceforth, benchmark predictor variables). Following, we run multivariate regressions of one-quarter ahead returns on each of our baseline predictor variables, first paired with each benchmark predictor variable, followed by pairing with all benchmark predictor variables simultaneously. Finally, we assess the in-and out-of-sample forecasting regression of both quarterly real returns and excess returns on each baseline and benchmark predictor variable.

Third, we assess the determinants or sources of information of the unobserved factors modelled in the FAVAR framework. Since the unobserved factors in $F_{t}$ are the additive elements in the estimation of 'other information' $\nu_{t}$, which is the primary focus of the paper, it will be useful to quantify how much each source of information contributes to the variation of $F_{t}$ Using the same estimation procedure, we estimate four subsets of $F_{t}$ extracted from the big data in $X_{t}$. These subsets include FM information only, accounting information only, investor and consumer sentiment information only, and macroeconomic information only. This enables us to conduct a simple variance decomposition analysis of the unobserved factors. 


\section{Tracking and Predictive Abilities of $\nu_{t}$}

We begin our empirical work by analyzing how well the baseline predictor variables track contemporaneous stock returns and predict future stock returns, relative to competing measures of $\nu_{t}$. Table 4.1 presents a comparison of alternative measures of 'other information' $\nu_{t}$ based on their ability to track variations of current S\&P 500 returns and predict one-quarter ahead S\&P 500 returns.

The composite tracking ability is measured by the a simple average of the absolute differences of both mean and standard deviation and AR(1) parameter. Predictor variables with lower scores indicate closer matching to the time-series properties of current returns. The composite predictive ability is also measured by a simple average of the correlation coefficient between the $\nu_{t}$ estimates and one-quarter ahead S\&P 500 returns and the Newey-West adjusted T-statistic for quarterly forecast regressions. Predictor variables with higher absolute averages demonstrate superior predictive power of future returns.

The baseline predictor variables emphatically demonstrate superior tracking, compared to the alternate value-relevant measures. The absolute differences of the mean and standard deviation show that they are more in line with the sample moments of current returns. The persistence parameter of a first-order autoregression suggests a faster mean-reversion for the baseline predictors by at least two-fold, relative to the alternate measures. Figure 4.1 displays a graphical illustration of the superior tracking of the baseline predictor variables, relative to the other measures. Figure 4.2 provides a closer look for the baseline predictors and current returns.

The baseline predictor variable scaled by market price only indicates a better predictive ability of future returns. The higher magnitude of the correlation coefficient and the Newey-West T-statistic yield a higher absolute composite average. It is important to note that the baseline predictor variables and the alternative measure, based on consensus analysts' earnings forecasts (DHS), are both negatively correlated with future returns. This possibly implies that positive (negative), value-relevant information about future profits of the S\&P 500 induces an appreciation (a depreciation) in market valuation. This subsequently makes equities appear less (more) risky thereby allowing the market to lower (raise) expected returns. As such, the positive corre- 
lation between future returns and the alternative VAR-derived $\nu_{t}$ is slightly puzzling. It seems to suggest that it is solely capturing the common linkages between the $\mathrm{B} / \mathrm{P}$ ratio and $\mathrm{ROE}$, which are both historically positively correlated with stock returns.

Overall, we find that the baseline predictors, particularly $\nu_{t} / p_{t}^{F A V A R}$, are able to track S\&P 500 returns and showcase predictive power for one-quarter ahead returns better than their counterparts. This provides evidence that the FAVAR-derived $\nu_{t}$ estimates are value-relevant to the behavior and predictability of stock returns. In the next subsection, we evaluate the forecasting performance of the baseline predictors and compare them to benchmark predictors.

\section{Forecasting Regressions}

Here, we assess the forecasting performance of the baseline predictors for stock returns and compare them to the performance of the competing benchmark predictors. Table 4.2 reports one-quarter ahead forecasts of the return on the S\&P 500. Each column represents results of an individual regression of a predictor variable, with the $\overline{R^{2}}$ measuring the forecasting ability. The coefficient estimates can be interpreted as the increase or decrease in basis points of the next quarter's returns given a 1 percentage point (100 basis point) change in a predictor variable.

Focusing on the benchmark predictors, only the $\mathrm{D} / \mathrm{P}$ ratio and ROE demonstrate statistically significant predictive power on future returns. The $\mathrm{D} / \mathrm{P}$ ratio (ROE) can predict nearly up to $5(2.5)$ percent of the variation in next quarter's stock returns. The p-values indicate that the coefficient estimates of those predictors are nonzero with at least a 90 percent probability. The $\mathrm{B} / \mathrm{P}$ and $\mathrm{E} / \mathrm{P}$ ratios explain a trivial percentage of future stock returns and have coefficient estimates that are not statistically different from zero.

The baseline predictors have marginally more predictive power for next quarter's stock returns than the benchmark predictors. The first baseline predictor $\nu_{t} / p_{t}^{F A V A R}$ appears to have the most forecasting ability of future returns at 5.65 percent. It explains from nearly 1 percentage point to 5.5 percentage points more than the leading and worst benchmark predictors, $\mathrm{D} / \mathrm{P}$ and $\mathrm{E} / \mathrm{P}$ ratios, respectively. It also has a statistically significant coefficient estimate of -0.305 with 95 percent confidence. This is in line with the correlation estimate for the predictor variable in Table 
4.1. The second baseline predictor, $\nu_{t} / b_{t}^{F A V A R}$, also performs better than the $\mathrm{B} / \mathrm{P}$ ratio, $\mathrm{E} / \mathrm{P}$ ratio, and ROE. It explains nearly 3 percent of the variation in quarterly returns. It also has a statistically significant coefficient estimate at -0.126 percent with 90 percent confidence. The marginal predictive power over the $\mathrm{B} / \mathrm{P}$ ratio, $\mathrm{E} / \mathrm{P}$ ratio, and $\mathrm{ROE}$ ranges from 44 basis points to nearly 300 basis points. By contrast, they do not perform better against the first baseline predictor and $\mathrm{D} / \mathrm{P}$ ratio.

Table 4.3 adds robustness to the univariate forecasting regression results in Table 4.2. Table 4.3 reports estimates from multivariate forecasting regressions of returns on our baseline predictors that include benchmark predictors as controls. Columns 1-8 are forecasting regressions of returns on a baseline predictor paired with each benchmark predictor. The remaining columns are forecasting regressions of returns on a baseline predictor paired with all benchmark predictors.

The results of the baseline predictors paired with the $\mathrm{B} / \mathrm{P}$ ratio, $\mathrm{E} / \mathrm{P}$ ratio, or $\mathrm{D} / \mathrm{P}$ ratio in columns 1-6 mirror the results in Table 4.2 with similar coefficient estimates and significance levels. The $\overline{R^{2}}$ are artificially inflated given an additional variable, but are in line with the sum of the $\overline{R^{2}}$ each baseline and benchmark predictor pairing in Table 4.2. This indicates that each pairing does not add predictive power.

The results of the baseline predictors paired with ROE in columns 7 and 8 are considerably different. With ROE as a control, both the $\overline{R^{2}}$ and the coefficient estimates of the baseline predictors increase in magnitude substantially. The first baseline predictor $\nu_{t} / p_{t}^{F A V A R}$ increases in magnitude by approximately 19 basis points to -0.491 from -0.3 . The $\overline{R^{2}}$ is 14.6 percent. The second baseline predictor $\nu_{t} / b_{t}^{F A V A R}$ increases in magnitude by approximately 14 basis points to -0.263 from -0.12 . The $\overline{R^{2}}$ is 12.6 percent. This is indicative of underestimated predictive power of the baseline predictors in the univariate case in Table 4.2.

Given the results in columns 7 and 8, we determine if the predictive power of the baseline predictors persist after controlling for all benchmark predictors considered in this analysis. Columns 9 and 10 display these aggregate results. The coefficient estimate of the first baseline predictor $\nu_{t} / p_{t}^{F A V A R}$ lowers in magnitude by 8 basis points to -0.419 . The coefficient estimate of 
the second baseline predictor $\nu_{t} / b_{t}^{F A V A R}$ lowers in magnitude by 6 basis points to -0.207 . However, they are still larger than the univariate case in Table 4.2 with 99 percent confidence. This provides further evidence that the predictive power of the baseline predictors are underestimated in the univariate case.

Tables 4.2 and 4.3 provide only in-sample results for real returns of the S\&P 500. Table 4.4 shows the in- and out-of-sample univariate forecasting performance of the predictor variables on quarterly real returns in Panel A and excess returns over the 90-day Treasury Bill in Panel B. Inspired by Goyal and Welch (2007), we conduct out-of-sample forecasts to minimize the lookahead bias of in-sample results and to compare our conditional forecasts with unconditional forecasts of the historical mean of returns. The out-of-sample (OoS) forecasts use only the data available up to the time at which the forecast is made. We let $e_{N}$ denote the vector of recursive OoS errors from the historical mean model and $e_{A}$ denote the vector of recursive OoS errors from the baseline predictor model. The OoS statistics are computed as

$$
\begin{gathered}
R^{2}=1-\frac{\mathrm{MSE}_{A}}{\mathrm{MSE}_{N}}, \\
\bar{R}^{2}=1-\left(1-R^{2}\right) \times\left(\frac{T-k}{T-1}\right),
\end{gathered}
$$

where $\mathrm{k}$ is the number of parameters in the baseline predictor model.

$$
\Delta \mathrm{RMSE}=\sqrt{\mathrm{MSE}_{N}}-\sqrt{\mathrm{MSE}_{A}}
$$

In Table 4.4, the in-sample results of the univariate regressions in Panel A are the same as in 4.1. The in-sample results of the univariate regressions in Panel B are similar to those of Panel A, with a few exceptions. The baseline predictors still show larger predictive power over many of the benchmark predictors, except the $\mathrm{D} / \mathrm{P}$ ratio. The $\mathrm{D} / \mathrm{P}$ ratio is the leading in-sample predictor of next quarter's excess returns of the S\&P 500 over the 90-day Treasury Bill with a $\overline{R^{2}}$ over 6 
percent. It also has a coefficient estimate of 0.024 with 99 percent confidence. Additionally, the $\mathrm{B} / \mathrm{P}$ ratio also has predictive power with a $\overline{R^{2}}$ of 2.39 percent. A significant improvement of its predictive power of real returns. It has a coefficient estimate of 0.015 with 90 percent confidence.

The OoS results in both Panel A and Panel B of Table 4.4 unequivocally showcase the inability of predictor variables to forecast quarterly real returns and excess returns. This is consistent with the arguments of Goyal and Welch (2007) who argue that the historical mean model forecasts future stock returns better than regressions of returns on predictor variables. In spite of this, in relative terms, the results demonstrate that the predictive power of our baseline predictors is larger than competing benchmark predictors. The $\overline{R^{2}}$ for the baseline predictors range from -2.47 percent to -0.82 percent. In contrast, the $\overline{R^{2}}$ for the benchmark predictors range from 5.45 percent to -14.54 percent. This provides evidence that the baseline predictors have closer potential to outperform the historical mean model.

In summary, the FAVAR-derived baseline predictors, which represent value-relevant measures, contain useful information extracted from big data to predict quarterly returns. This result is robust to additional predictor variables as controls.

\section{Variance Decomposition of the Unobserved Factors}

Given the forecasting performance of the baseline predictors in the previous section, within a FAVAR system, we conduct a forecast error variance decomposition of the estimated unobserved factors $F_{t}$ using big data in $X_{t}$. We do this to quantify the contribution of each source of information to the variation of $F_{t}$. The sources of information come from financial market data, accounting data, investor and consumer sentiment data, and macroeconomic data. Table 4.5 displays the variance decomposition results for $F_{t}$. We find that a one standard deviation shock to factors summarized by financial market data contribute to nearly 60 percent of the variation in $F_{t}$ after one quarter. For the same horizon, a shock to accounting and macroeconomic factors make up almost 25 and 15 percent of the variation of $F_{t}$, respectively. A shock to investor and consumer sentiment factors are negligible. The relative contribution of each factor, except accounting, begins to decay marginally by the second quarter. The contribution of an account- 
ing shock becomes more pronounced after one quarter. It peaks after the fourth quarter before decaying marginally in longer horizons.

Overall, this result showcases that the value-relevancy the FAVAR-derived estimates of $\nu_{t}$ predominantly stems from financial market and accounting information at over 80 percent consistently and persists over time. This is not surprising considering that such data would contain more forward-looking metrics that are useful for stock return tracking and predictability.

\subsection{Conclusions}

This paper applies a FAVAR model, developed in Bernanke et al. (2005), to estimate 'other information' $\nu_{t}$, as postulated in Ohlson (1995). Ohlson (1995) defines 'other information' $\nu_{t}$ as a summary of value-relevant information about events and their effect on future profitability. This implies a potential to track contemporaneous stock returns and predict future returns. Past work in the residual income literature proxies $\nu_{t}$ with Wall Street analysts' earnings to capture unobserved value-relevant information. Additionally, previous literature of stock return predictability has found that traditional valuation ratios have weak predictive power for future returns.

We contribute to both branches of literature with a FAVAR approach because it enables us to use a rich set of data to create a reliable value-relevant measure of 'other information' $\nu_{t}$, which is important to stock valuation according to Ohlson's theory. We estimate the FAVAR with use of a two-step principal components estimation approach to extract the unobserved factors from 78 informational variables from financial market, accounting, investor and consumer sentiment, and macroeconomic data. A summary of such rich data captured by few unobserved factors could provide useful information for stock return predictability.

We find that our measure of $\nu_{t}$ derived in the FAVAR framework is a favorable measure as it has closely matching sample moments to current stock returns, a faster rate of mean-reversion, and predicts future returns better, relative to alternative measures employed in the analysis. We also find that our estimate of $\nu_{t}$ has more predictive power for next period real returns and excess returns, both in- and out-of-sample, compared to traditional valuation ratios. Additionally, 
given the promising performance of our $\nu_{t}$ measure, we conduct a variance decomposition of the unobserved factors to learn about the determinants or sources of information. We show that the majority of information stems from financial market and accounting data and persists over time. 
Table 4.1: Tracking and Predictive Ability of Competing Measures of $\nu_{t}$

\begin{tabular}{|c|c|c|c|c|c|c|c|c|c|c|c|}
\hline \multirow{4}{*}{ Variable } & \multicolumn{8}{|c|}{ Tracking Ability } & \multicolumn{3}{|c|}{ Predictive Ability } \\
\hline & Mean & $\overline{r_{t}}$ & |Diff $\mid$ & Std. Dev. & $S D^{r_{t}}$ & |Diff $\mid$ & $\operatorname{AR}(1)$ & Composite & Corr $\mathrm{w} / r_{t+1}$ & Newey-West & Composite \\
\hline & & & & & & & Parameter & Tracking Ability & & T-statistic & Predictive \\
\hline & & & & & & & & & & & Ability \\
\hline$\nu_{t} / p_{t}^{F A V A R}$ & 0.006 & 0.041 & 0.035 & 0.069 & 0.081 & 0.012 & 0.418 & 0.155 & -0.258 & -2.312 & -1.285 \\
\hline$\nu_{t} / p_{t}^{V A R}$ & -0.193 & 0.041 & 0.234 & 0.442 & 0.081 & 0.361 & 0.935 & 0.510 & 0.248 & 1.834 & 1.041 \\
\hline$\nu_{t} / p_{t}^{D H S}$ & -0.154 & 0.041 & 0.195 & 0.430 & 0.081 & 0.349 & 0.933 & 0.492 & -0.190 & -1.181 & -0.685 \\
\hline$\nu_{t} / b_{t}^{F A V A R}$ & 0.011 & 0.041 & 0.030 & 0.128 & 0.081 & 0.048 & 0.309 & 0.129 & -0.200 & -1.998 & -1.099 \\
\hline$\nu_{t} / b_{t}^{V A R}$ & -0.226 & 0.041 & 0.267 & 0.746 & 0.081 & 0.666 & 0.949 & 0.627 & 0.244 & 2.021 & 1.133 \\
\hline$\nu_{t} / b_{t}^{D H S}$ & -0.221 & 0.041 & 0.262 & 0.569 & 0.081 & 0.488 & 0.932 & 0.561 & -0.149 & -1.002 & -0.575 \\
\hline
\end{tabular}

This table provides a comparison of competing measures of $\nu_{t}$ estimates based on their tracking ability of contemporaneous $\mathrm{S} \& \mathrm{P} 500$ return variations over time and their predictive ability of next quarter's S\&P 500 return. The composite tracking ability is the average measure of the absolute differences of the mean and standard deviations between a predictor variable and index returns and the AR(1) persistence parameter. Variables with lower scores closely match with the sample moments of the index return. The composite predictive ability is the average measure of the correlation coefficient between a predictor variable and one-quarter ahead return and the Newey-West adjusted T-statistic for one-quarter ahead stock return. Variables with higher scores in terms of magnitude have predictive power. 
Table 4.2: Forecasting Quarterly S\&P 500 Returns: Univariate Regressions

\begin{tabular}{|c|c|c|c|c|c|c|}
\hline \multicolumn{7}{|c|}{ S\&P 500 Returns: $r_{t+1}$} \\
\hline & (1) & (2) & (3) & (4) & (5) & (6) \\
\hline \multirow[t]{2}{*}{ Intercept } & $0.030^{* * *}$ & $0.039^{* * *}$ & $0.002^{* * *}$ & $0.039^{* * *}$ & $0.040^{* * *}$ & $0.056^{* * *}$ \\
\hline & $(0.000)$ & $(0.000)$ & $(0.000)$ & $(0.000)$ & $(0.000)$ & $(0.000)$ \\
\hline \multirow[t]{2}{*}{$\mathrm{B} / \mathrm{P}$} & 0.010 & & & & & \\
\hline & $(0.214)$ & & & & & \\
\hline \multirow[t]{2}{*}{$\mathrm{E} / \mathrm{P}$} & & 0.009 & & & & \\
\hline & & $(0.275)$ & & & & \\
\hline \multirow[t]{2}{*}{$\mathrm{D} / \mathrm{P}$} & & & $0.021^{* *}$ & & & \\
\hline & & & $(0.020)$ & & & \\
\hline \multirow[t]{2}{*}{ ROE } & & & & $0.015^{*}$ & & \\
\hline & & & & $(0.068)$ & & \\
\hline \multirow[t]{2}{*}{$\nu_{t} / p_{t}^{F A V A R}$} & & & & & $-0.305^{* *}$ & \\
\hline & & & & & $(0.012)$ & \\
\hline \multirow[t]{2}{*}{$\nu_{t} / b_{t}^{F A V A R}$} & & & & & & $-0.126^{*}$ \\
\hline & & & & & & $(0.053)$ \\
\hline$\overline{R^{2}}$ & $0.60 \%$ & $0.22 \%$ & $4.74 \%$ & $2.52 \%$ & $5.65 \%$ & $2.96 \%$ \\
\hline \multicolumn{7}{|c|}{$\begin{array}{l}\text { This table provides estimates of simple one-quarter ahead univariate fore- } \\
\text { cast regressions of } \mathrm{S} \& \mathrm{P} 500 \text { market returns. The } \mathrm{B} / \mathrm{P} \text { ratio, } \mathrm{E} / \mathrm{P} \text {, ratio, } \mathrm{D} / \mathrm{P} \\
\text { ratio, and ROE compose of benchmark predictor variables. } \nu_{t} / p_{t}^{F} A V A R \text {, and } \\
\nu_{t} / b_{t}^{F A V A R} \text { make up the baseline predictor variables. The coefficient esti- } \\
\text { mates can be interpreted as the increase or decrease in basis points of the } \\
\text { next quarter's returns given a } 1 \text { percentage point (100 basis point) change in } \\
\text { a predictor variable. Numbers in parentheses are } \mathrm{p} \text {-values. }{ }^{*} \mathrm{p}<0.1 ;{ }^{* *} \mathrm{p}<0.05 \text {; }\end{array}$} \\
\hline
\end{tabular}


Table 4.3: Forecasting Quarterly S\&P 500 Returns: Multivariate Regressions

\begin{tabular}{|c|c|c|c|c|c|c|c|c|c|c|}
\hline \multicolumn{11}{|c|}{ S\&P 500 Returns: $r_{t+1}$} \\
\hline & (1) & (2) & (3) & (4) & (5) & (6) & (7) & (8) & (9) & (10) \\
\hline \multirow[t]{2}{*}{ Intercept } & $0.040^{* * *}$ & $0.040^{* * *}$ & $0.041^{* * *}$ & $0.040^{* * *}$ & $0.042^{* * *}$ & $0.041^{* * *}$ & $0.041^{* * *}$ & $0.041^{* * *}$ & $0.043^{* * *}$ & $0.042^{* * *}$ \\
\hline & $(0.000)$ & $(0.000)$ & $(0.000)$ & $(0.000)$ & $(0.000)$ & $(0.000)$ & $(0.000)$ & $(0.000)$ & $(0.000)$ & $(0.000)$ \\
\hline \multirow[t]{2}{*}{$\mathrm{B} / \mathrm{P}$} & 0.010 & 0.009 & & & & & & & 0.007 & 0.009 \\
\hline & $(0.216)$ & $(0.276)$ & & & & & & & $(0.559)$ & $(0.495)$ \\
\hline \multirow[t]{2}{*}{$\mathrm{E} / \mathrm{P}$} & & & 0.009 & 0.009 & & & & & -0.018 & -0.018 \\
\hline & & & $(0.275)$ & $(0.304)$ & & & & & $(0.133)$ & $(0.125)$ \\
\hline \multirow[t]{2}{*}{$\mathrm{D} / \mathrm{P}$} & & & & & $0.020^{* *}$ & $0.019^{* *}$ & & & $0.028^{* *}$ & $0.026^{* *}$ \\
\hline & & & & & $(0.028)$ & $(0.037)$ & & & $(0.016)$ & $(0.030)$ \\
\hline \multirow[t]{2}{*}{ ROE } & & & & & & & 0.010 & 0.012 & $0.032^{* * *}$ & $0.034^{* * *}$ \\
\hline & & & & & & & $(0.179)$ & $(0.142)$ & $(0.006)$ & $(0.005)$ \\
\hline \multirow[t]{2}{*}{$\nu_{t} / p_{t}^{F A V A R}$} & $-0.302^{* *}$ & & $-0.303^{* *}$ & & $-0.285^{* *}$ & & $-0.491^{* * *}$ & & $-0.419^{* * *}$ & \\
\hline & $(0.012)$ & & $(0.012)$ & & $(0.017)$ & & $(0.000)$ & & $(0.001)$ & \\
\hline \multirow[t]{2}{*}{$\nu_{t} / b_{t}^{F A V A R}$} & & $-0.120^{*}$ & & $-0.124^{*}$ & & -0.106 & & $-0.263^{* * *}$ & & $-0.207^{* * *}$ \\
\hline & & $(0.067)$ & & $(0.059)$ & & $(0.102)$ & & $(0.001)$ & & $(0.009)$ \\
\hline$\overline{R^{2}}$ & $6.21 \%$ & $3.17 \%$ & $5.86 \%$ & $3.03 \%$ & $9.59 \%$ & $6.49 \%$ & $14.60 \%$ & $12.60 \%$ & $21.80 \%$ & $18.80 \%$ \\
\hline
\end{tabular}

This table provides estimates of one-quarter ahead multivariate forecast regressions of S\&P 500 market returns. The B/P ratio, E/P, ratio, D/P ratio, and ROE compose of benchmark predictor variables. $\nu_{t} / p_{t}^{F A V A R}$, and $\nu_{t} / b_{t}^{F A V A R}$ make up the baseline predictor variables. Columns 1-8 are estimates for one-quarter ahead S\&P 500 return regressions on each baseline predictor variable paired with each benchmark predictor as a control. Columns 9 and 10 are estimates for one-quarter ahead S\&P 500 return regressions on each baseline predictor variable paired with all benchmark predictors as controls. The coefficient estimates can be interpreted as the increase or decrease in basis points of the next quarter's returns given a 1 percentage point (100 basis point) change in a predictor variable. Numbers in parentheses are $\mathrm{p}$-values. ${ }^{*} \mathrm{p}<0.1 ;{ }^{* *} \mathrm{p}<0.05$; ${ }^{* * *} \mathrm{p}<0.01$ 
Table 4.4: Forecasting Quarterly S\&P 500 Returns: In-sample and Outof-Sample

\begin{tabular}{|c|c|c|c|c|}
\hline \multirow[b]{3}{*}{ Variable } & & & \multicolumn{2}{|c|}{ Forecasts begin 24 quarters after sample } \\
\hline & \multicolumn{2}{|c|}{ IS } & \multicolumn{2}{|c|}{ OoS } \\
\hline & $\hat{\beta}$ & $\overline{R^{2}}$ & $\overline{R^{2}}$ & $\Delta$ RMSE \\
\hline \multicolumn{5}{|c|}{ Panel A: Real Returns: 1994 Q1-2018 Q4 } \\
\hline$\nu_{t} / p_{t}^{F A V A R}$ & $\begin{array}{c}-0.305^{* *} \\
(0.012)\end{array}$ & $5.65 \%$ & $-1.21 \%$ & $-0.05 \%$ \\
\hline$\nu_{t} / b_{t}^{F A V A R}$ & $\begin{array}{l}-0.126^{*} \\
(0.053)\end{array}$ & $2.96 \%$ & $-0.82 \%$ & $0.01 \%$ \\
\hline $\mathrm{B} / \mathrm{P}$ & $\begin{array}{l}0.010 \\
(0.214)\end{array}$ & $0.60 \%$ & $-14.54 \%$ & $-0.54 \%$ \\
\hline $\mathrm{E} / \mathrm{P}$ & $\begin{array}{l}0.009 \\
(0.275)\end{array}$ & $0.22 \%$ & $-6.63 \%$ & $-0.23 \%$ \\
\hline $\mathrm{D} / \mathrm{P}$ & $\begin{array}{l}0.021^{* *} \\
(0.020)\end{array}$ & $4.74 \%$ & $-5.45 \%$ & $-0.18 \%$ \\
\hline \multirow[t]{2}{*}{ ROE } & $\begin{array}{l}0.015^{*} \\
(0.068)\end{array}$ & 0.025 & $-6.83 \%$ & $-0.23 \%$ \\
\hline & Panel B & Excess & Returns: 1 & $8 \mathrm{Q} 4$ \\
\hline$\nu_{t} / p_{t}^{F A V A R}$ & $\begin{array}{c}-0.311^{* *} \\
(0.011)\end{array}$ & $5.86 \%$ & $-2.47 \%$ & $-0.05 \%$ \\
\hline$\nu_{t} / b_{t}^{F A V A R}$ & $\begin{array}{c}-0.132^{* *} \\
(0.044)\end{array}$ & $3.32 \%$ & $-1.81 \%$ & $-0.03 \%$ \\
\hline $\mathrm{B} / \mathrm{P}$ & $\begin{array}{l}0.015^{*} \\
(0.074)\end{array}$ & $2.39 \%$ & $-14.40 \%$ & $-0.54 \%$ \\
\hline $\mathrm{E} / \mathrm{P}$ & $\begin{array}{c}0.010 \\
(0.263)\end{array}$ & $0.29 \%$ & $-7.78 \%$ & $-0.27 \%$ \\
\hline $\mathrm{D} / \mathrm{P}$ & $\begin{array}{c}0.024^{* * *} \\
(0.008)\end{array}$ & $6.29 \%$ & $-6.22 \%$ & $-0.21 \%$ \\
\hline ROE & $\begin{array}{l}0.012 \\
(0.148)\end{array}$ & $1.20 \%$ & $-10.26 \%$ & $-0.37 \%$ \\
\hline \multicolumn{5}{|c|}{$\begin{array}{l}\text { This table provides estimates of one-quarter ahead univariate in-sample } \\
\text { and out-of-sample forecast regressions of } \mathrm{S} \& \mathrm{P} 500 \text { real market returns in } \\
\text { Panel A and excess market returns in Panel B. The B/P ratio, E/P, ratio, D/P } \\
\text { ratio, and ROE compose of benchmark predictor variables. } \nu_{t} / p_{t}^{F A V A R} \text {, } \\
\text { and } \nu_{t} / b_{t}^{F A V A R} \text { make up the baseline predictor variables. } \triangle \mathrm{RMSE} \text { is the } \\
\text { RMSE (root mean square error) difference between the historical mean } \\
\text { model and the conditional forecast regressions for the same forecast pe- } \\
\text { riod. Positive numbers signify superior out-of-sample conditional fore- } \\
\text { casts. The OoS } \overline{R^{2}} \text { is defined in Equations ( } 8 \text { ) and (9). The coefficient esti- } \\
\text { mates can be interpreted as the increase or decrease in basis points of the } \\
\text { next quarter's returns given a } 1 \text { percentage point (100 basis point) change } \\
\text { in a predictor variable. Numbers in parentheses are p-values. }{ }^{*} \mathrm{p}<0.1 \text {; } \\
{ }^{* *} \mathrm{p}<0.05 ;{ }^{* * *} \mathrm{p}<0.01 \text {. }\end{array}$} \\
\hline
\end{tabular}


Table 4.5: Variance Decomposition of $F_{t}$

\begin{tabular}{ccccc}
\hline \hline & \multicolumn{5}{c}{ Variance Decomposition } \\
\hline Forecast Horizon & $F_{t}^{F M}$ & $F_{t}^{\text {Acct }}$ & $F_{t}^{I C S}$ & $F_{t}^{\text {Macro }}$ \\
\hline 1 & 0.589 & 0.244 & 0.017 & 0.137 \\
2 & 0.494 & 0.358 & 0.014 & 0.095 \\
3 & 0.402 & 0.457 & 0.012 & 0.072 \\
4 & 0.368 & 0.482 & 0.010 & 0.067 \\
12 & 0.288 & 0.439 & 0.009 & 0.077 \\
20 & 0.283 & 0.437 & 0.009 & 0.079 \\
\hline
\end{tabular}

This table provides decomposition of the variation of the unobserved factor $F_{t}$, as estimated by principal components, into subsets of unobserved components by information category. $F_{t}^{F M}$ is the unobserved factor for financial market; $F_{t}^{A c c t}$ is the unobserved factor for accounting; $F_{t}^{I C S}$ is the unobserved factor for investor and consumer sentiment; and $F_{t}^{\text {Macro }}$ is the unobserved factor for macroeconomic variables. Each shock represents a 1 standard deviation above the mean for each factor. 


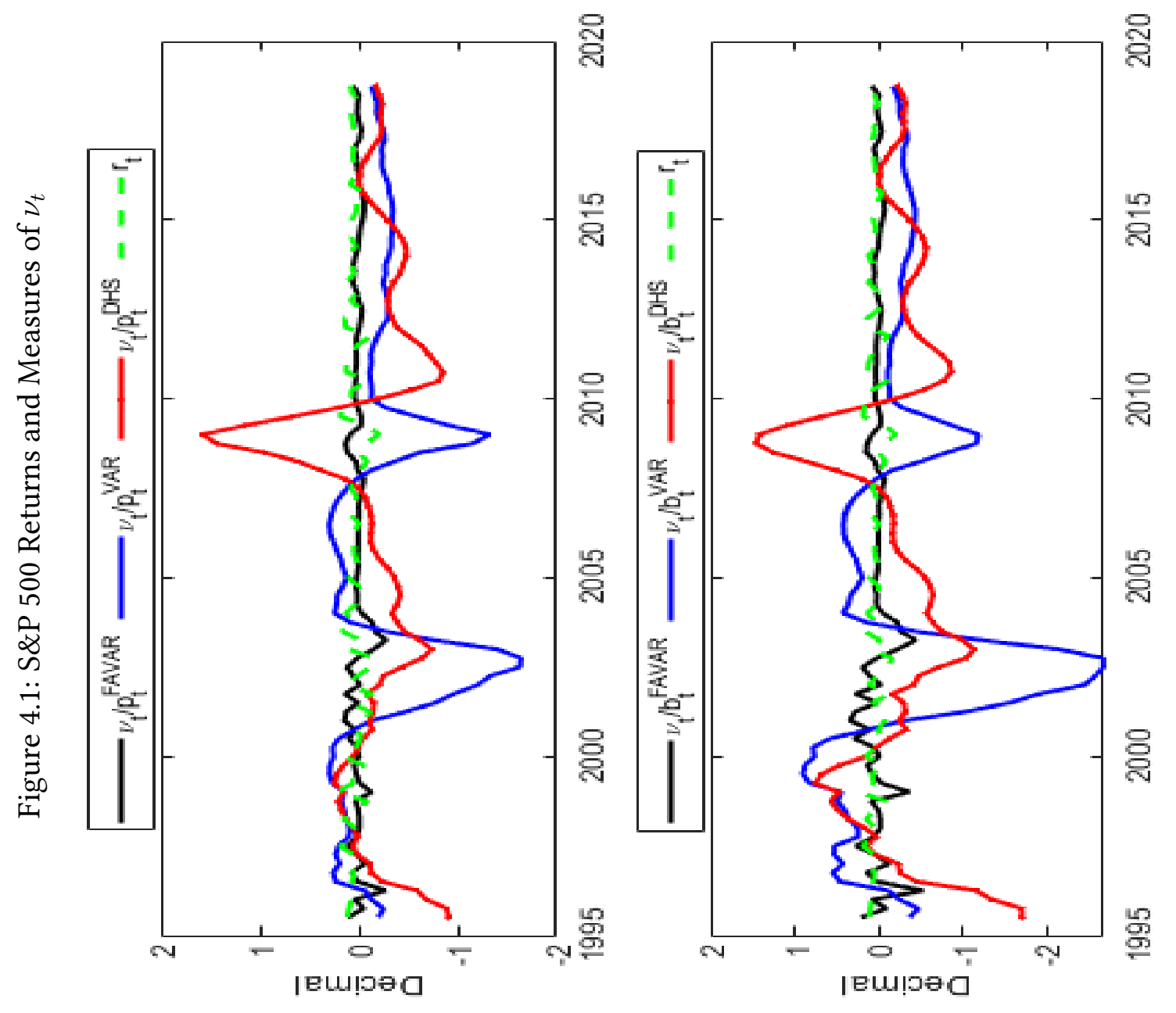




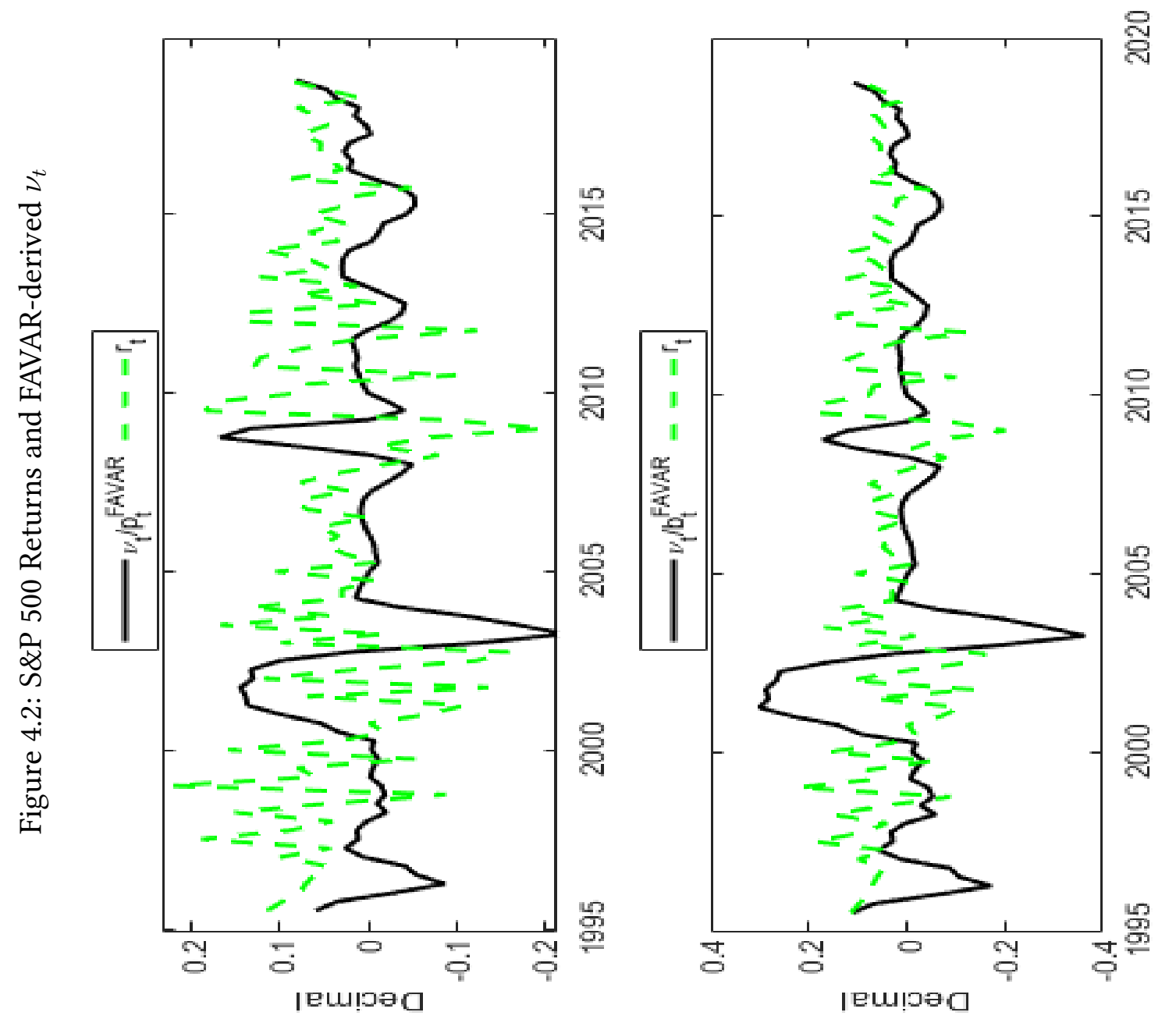




\subsection{Appendix}

Following BBE, the transformation codes are the following: 1 (no transformation); 2 (first difference); 4 (logarithm); 5 (first difference of logarithm.) An asterisk ${ }^{*}$, next to the mnemonic, denotes a variable assumed to be slow-moving in the estimation.

Table 4.6: Data Description

\begin{tabular}{|c|c|c|c|c|}
\hline \# & Name & Category & Sub-category & Tcode \\
\hline 1 & Payout Ratio & FM & Valuation Metrics & 2 \\
\hline 2 & Dividend Yield & FM & Valuation Metrics & 2 \\
\hline 3 & $\mathrm{D} / \mathrm{P}$ & $\mathrm{FM}$ & Valuation Metrics & 2 \\
\hline 4 & $\mathrm{E} / \mathrm{P}$ & FM & Valuation Metrics & 2 \\
\hline 5 & Sales/P & $\mathrm{FM}$ & Valuation Metrics & 2 \\
\hline 6 & EPS Growth & $\mathrm{FM}$ & Valuation Metrics & 2 \\
\hline 7 & EV/Oper Inc Before Depr & FM & Valuation Metrics & 2 \\
\hline 8 & Sales/EV & FM & Valuation Metrics & 2 \\
\hline 9 & $\mathrm{~B} / \mathrm{EV}$ & FM & Valuation Metrics & 2 \\
\hline 10 & Sharpe Ratio & FM & Valuation Metrics & 2 \\
\hline 11 & Small Minus Big (SMB) Risk Premium & FM & FF5 & 2 \\
\hline 12 & High Minus Low (HML) Risk Premium & FM & FF5 & 2 \\
\hline 13 & Robust Minus Weak (RMW) Risk Premium & FM & FF5 & 2 \\
\hline 14 & Conservative Minus Aggressive (CMA) Risk Premium & FM & FF5 & 2 \\
\hline 15 & Market Risk Premium (Mkt-RF) & FM & FF5 & 2 \\
\hline 16 & Small and Low E/P (SMALL LoEP) Risk Premium & $\mathrm{FM}$ & Size and $\mathrm{E} / \mathrm{P}$ & 2 \\
\hline 17 & Medium and 30th Pctl EP (ME1 EP2) Risk Premium & $\mathrm{FM}$ & Size and E/P & 2 \\
\hline 18 & Small and High E/P (SMALL HiEP) Risk Premium & FM & Size and $\mathrm{E} / \mathrm{P}$ & 2 \\
\hline 19 & Big and Low E/P (BIG LoEP) Risk Premium & FM & Size and $\mathrm{E} / \mathrm{P}$ & 2 \\
\hline 20 & Medium and 70th Pctl E/P (ME2 EP2) Risk Premium & FM & Size and $\mathrm{E} / \mathrm{P}$ & 2 \\
\hline 21 & Big and High E/P (BIG HiEP) Risk Premium & FM & Size and $\mathrm{E} / \mathrm{P}$ & 2 \\
\hline 22 & Small and Low CF/P (SMALL LoCFP) Risk Premium & $\mathrm{FM}$ & Size and $\mathrm{CF} / \mathrm{P}$ & 2 \\
\hline 23 & Medium and 30th Pctl CF/P (ME1 CFP2) Risk Premium & $\mathrm{FM}$ & Size and $\mathrm{CF} / \mathrm{P}$ & 2 \\
\hline 24 & Small and High $\mathrm{CF} / \mathrm{P}$ (SMALL HiCFP) Risk Premium & FM & Size and $\mathrm{CF} / \mathrm{P}$ & 2 \\
\hline
\end{tabular}




\begin{tabular}{|c|c|c|c|c|}
\hline 25 & Big and Low CF/P (BIG LoCFP) Risk Premium & FM & Size and $\mathrm{CF} / \mathrm{P}$ & 2 \\
\hline 26 & Medium and 70th Pctl CF/P (ME2 CFP2) Risk Premium & FM & Size and $\mathrm{CF} / \mathrm{P}$ & 2 \\
\hline 27 & Big and High CF/P (BIG HiCFP) Risk Premium & FM & Size and CF/P & 2 \\
\hline 28 & Small and Low D/P (SMALL LoDP) Risk Premium & FM & Size and $\mathrm{D} / \mathrm{P}$ & 2 \\
\hline 29 & Medium and 30th Pctl D/P (ME1 DP2) Risk Premium & FM & Size and $\mathrm{D} / \mathrm{P}$ & 2 \\
\hline 30 & Small and High D/P (SMALL HiDP) Risk Premium & FM & Size and $\mathrm{D} / \mathrm{P}$ & 2 \\
\hline 31 & Big and Low D/P (BIG LoDP) Risk Premium & FM & Size and $\mathrm{D} / \mathrm{P}$ & 2 \\
\hline 32 & Medium and 70th Pctl D/P (ME2 DP2) Risk Premium & FM & Size and $\mathrm{D} / \mathrm{P}$ & 2 \\
\hline 33 & Big and High D/P (BIG HiDP) Risk Premium & FM & Size and $\mathrm{D} / \mathrm{P}$ & 2 \\
\hline 34 & Momentum Risk Premium & FM & Momentum Factor & 2 \\
\hline 35 & Short-term Reversal Risk Premium & FM & Short-Term Reversal Factor & 2 \\
\hline 36 & Gross Profit Ratio & Accounting Ratios & Profitability & 2 \\
\hline 37 & Operating Profit Ratio & Accounting Ratios & Profitability & 2 \\
\hline 38 & Net Profit Ratio & Accounting Ratios & Profitability & 2 \\
\hline 39 & ROA & Accounting Ratios & Profitability & 2 \\
\hline 40 & D/E Ratio & Accounting Ratios & Solvency & 2 \\
\hline 41 & Debt Ratio & Accounting Ratios & Solvency & 2 \\
\hline 42 & Proprietary Ratio & Accounting Ratios & Solvency & 2 \\
\hline 43 & Int. Coverage Ratio & Accounting Ratios & Solvency & 2 \\
\hline 44 & LT Debt to Equity & Accounting Ratios & Solvency & 2 \\
\hline 45 & Current Ratio & Accounting Ratios & Liquidity & 2 \\
\hline 46 & Quick Ratio & Accounting Ratios & Liquidity & 2 \\
\hline 47 & Cash Ratio & Accounting Ratios & Liquidity & 2 \\
\hline 48 & Operating CF Ratio & Accounting Ratios & Liquidity & 2 \\
\hline 49 & Working Capital Ratio & Accounting Ratios & Efficiency & 2 \\
\hline 50 & Inventory Turnover Ratio & Accounting Ratios & Efficiency & 2 \\
\hline 51 & Asset Turnover Ratio & Accounting Ratios & Efficiency & 2 \\
\hline 52 & DSO Ratio & Accounting Ratios & Efficiency & 2 \\
\hline 53 & ROIC & Accounting Ratios & Efficiency & 2 \\
\hline 54 & CF ROIC & Accounting Ratios & Efficiency & 2 \\
\hline 55 & Mkt Cap to GDP & Macro & Economic Activity & 2 \\
\hline
\end{tabular}




\begin{tabular}{|c|c|c|c|c|}
\hline 56 & GDP Growth \%* & Macro & Economic Activity & 2 \\
\hline 57 & GDPPC Growth \%* & Macro & Economic Activity & 2 \\
\hline 58 & Cons. Growth \%* & Macro & Economic Activity & 2 \\
\hline 59 & Production Growth \%* & Macro & Economic Activity & 2 \\
\hline 60 & Capacity Utilization $\% *$ & Macro & Economic Activity & 2 \\
\hline 61 & Unemployment $\% *$ & Macro & Employment \& Wages & 2 \\
\hline 62 & Nat. Rate of Unemployment $\% *$ & Macro & Employment \& Wages & 2 \\
\hline 63 & Wage Growth $\% *$ & Macro & Employment \& Wages & 2 \\
\hline 64 & Inflation $\% *$ & Macro & Inflation \& Expectations & 2 \\
\hline 65 & Inflation Expectation \% & Macro & Inflation \& Expectations & 2 \\
\hline 66 & 10Y3M Spread \% & Macro & Interest Rates & 2 \\
\hline 67 & 10Y2M Spread \% & Macro & Interest Rates & 2 \\
\hline 68 & Momentum Indicator & Macro & Labor Market Conditions & 2 \\
\hline 69 & Level of Activity Indicator & Macro & Labor Market Conditions & 2 \\
\hline 70 & All-Trans House Price Growth & Macro & Housing Market Conditions & 2 \\
\hline 71 & Homer Ownership \% & Macro & Housing Market Conditions & 2 \\
\hline 72 & House Supply Growth & Macro & Housing Market Conditions & 2 \\
\hline 73 & VIX Growth & Macro & Market Fear Gauge & 2 \\
\hline 74 & Consumer Sentiment Growth & Investor \& Consumer Sentiment & Consumer Sentiment & 2 \\
\hline 75 & 1Y Confidence Growth & Investor \& Consumer Sentiment & Investor Sentiment & 2 \\
\hline 76 & Buys On Dips Confidence Growth & Investor \& Consumer Sentiment & Investor Sentiment & 2 \\
\hline 77 & Crash Confidence Growth & Investor \& Consumer Sentiment & Investor Sentiment & 2 \\
\hline 78 & Valuation Confidence Growth & Investor \& Consumer Sentiment & Investor Sentiment & 2 \\
\hline
\end{tabular}




\section{BIBLIOGRAPHY}

[AB19] Faisal M Awwal and Prasad V Bidarkota. A state space framework for the residual income valuation model of stock prices. Available at SSRN 3466181, 2019.

[AL01] Andrew Ang and Jun Liu. A general affine earnings valuation model. Review of Accounting Studies, 6(4):397-425, 2001.

[AS93] Lucy F Ackert and Brian F Smith. Stock price volatility, ordinary dividends, and other cash flows to shareholders. The fournal of Finance, 48(4):1147-1160, 1993.

[BBE05] Ben S Bernanke, Jean Boivin, and Piotr Eliasz. Measuring the effects of monetary policy: a factor-augmented vector autoregressive (favar) approach. The Quarterly fournal of Economics, 120(1):387-422, 2005.

[BBHL99] Mary E Barth, William H Beaver, John RM Hand, and Wayne R Landsman. Accruals, cash flows, and equity values. Review of Accounting Studies, 4(34):205-229, 1999.

[BC05] Gurdip Bakshi and Zhiwu Chen. Stock valuation in dynamic economies. fournal of Financial Markets, 8(2):111-151, 2005.

[BD07] Prasad V Bidarkota and Brice V Dupoyet. Intrinsic bubbles and fat tails in stock prices: A note. Macroeconomic Dynamics, 11(3):405-422, 2007.

[BDM09] Prasad V Bidarkota, Brice V Dupoyet, and J Huston McCulloch. Asset pricing with incomplete information and fat tails. Journal of Economic Dynamics and Control, 33(6):1314-1331, 2009.

[BGM09] Jean Boivin, Marc P Giannoni, and Ilian Mihov. Sticky prices and monetary policy: Evidence from disaggregated us data. American Economic Review, 99(1):350-84, 2009.

[BGS18] Claude Bergeron, Jean-Pierre Gueyie, and Komlan Sedzro. Consumption, residual income valuation, and long-run risk. Journal of Theoretical Accounting Research, 13(2):1-32, 2018.

[BJ02] Gurdip Bakshi and Nengjiu Ju. Book values, earnings, and market valuations. Unpublished working paper, University of Maryland, 2002. 
[BK13] Jörg Breitung and Robinson Kruse. When bubbles burst: econometric tests based on structural breaks. Statistical Papers, 54(4):911-930, 2013.

[Bla79] Olivier Jean Blanchard. Speculative bubbles, crashes and rational expectations. Economics Letters, 3(4):387-389, 1979.

[BLL16] Jushan Bai, Kunpeng Li, and Lina Lu. Estimation and inference of favar models. Journal of Business \& Economic Statistics, 34(4):620-641, 2016.

[BM12] Sudhakar Balachandran and Partha Mohanram. Using residual income to refine the relationship between earnings growth and stock returns. Review of Accounting Studies, 17(1):134-165, 2012.

[BN02] Jushan Bai and Serena Ng. Determining the number of factors in approximate factor models. Econometrica, 70(1):191-221, 2002.

[BT19] Ruby Brownen-Trinh. Effects of winsorization: The cases of forecasting nongaap and gaap earnings. Journal of Business Finance \& Accounting, 46(12):105-135, 2019.

[Bur98] Craig Burnside. Solving asset pricing models with gaussian shocks. fournal of Economic Dynamics and Control, 22(3):329-340, 1998.

[BW82] Olivier J Blanchard and Mark W Watson. Bubbles, rational expectations and financial markets. Technical report, National Bureau of Economic Research, 1982.

[BY04] Ravi Bansal and Amir Yaron. Risks for the long run: A potential resolution of asset pricing puzzles. The fournal of Finance, 59(4):1481-1509, 2004.

[CC16] Tsangyao Chang and Yifei Cai. Do bubbles exist in chinese share markets? International Review of Accounting, Banking \& Finance, 8, 2016.

[CF15] Eng-Tuck Cheah and John Fry. Speculative bubbles in bitcoin markets? an empirical investigation into the fundamental value of bitcoin. Economics Letters, 130:32-36, 2015.

[CLY18] Shaen Corbet, Brian Lucey, and Larisa Yarovaya. Datestamping the bitcoin and ethereum bubbles. Finance Research Letters, 26:81-88, 2018. 
[CS87] John Y Campbell and Robert J Shiller. Cointegration and tests of present value models. Journal of Political Economy, 95(5):1062-1088, 1987.

[CZ07] Peter Chen and Guochang Zhang. How do accounting variables explain stock price movements? theory and evidence. Fournal of Accounting and Economics, 43(2-3):219-244, 2007.

[DG83] Behzad T Diba and Herschel I Grossman. Rational asset price bubbles. Technical report, National Bureau of Economic Research, 1983.

[DG88a] Behzad T Diba and Herschel I Grossman. Explosive rational bubbles in stock prices? The American Economic Review, 78(3):520-530, 1988.

[DG88b] Behzad T Diba and Herschel I Grossman. The theory of rational bubbles in stock prices. The Economic Journal, 98(392):746-754, 1988.

[DHS99] Patricia M Dechow, Amy P Hutton, and Richard G Sloan. An empirical assessment of the residual income valuation model. Fournal of Accounting and Economics, 26(1-3):1-34, 1999.

[FF93] Eugene F Fama and Kenneth R French. Common risk factors in the returns on stocks and bonds. Fournal of Financial Economics, 33(1):3-56, 1993.

[FF95] Eugene F Fama and Kenneth R French. Size and book-to-market factors in earnings and returns. The fournal of Finance, 50(1):131-155, 1995.

[FF96] Eugene F Fama and Kenneth R French. Multifactor explanations of asset pricing anomalies. The fournal of Finance, 51(1):55-84, 1996.

[FF98] Eugene F Fama and Kenneth R French. Value versus growth: The international evidence. The fournal of Finance, 53(6):1975-1999, 1998.

[FF01] Eugene F Fama and Kenneth R French. Disappearing dividends: changing firm characteristics or lower propensity to pay? Fournal of Financial Economics, 60(1):3-43, 2001.

[Fis07] Jill E Fisch. Does analyst independence sell investors short. UCLA L. Rev., 55:39, 2007. 
[FM19] Cristiana Fiorelli and Valentina Meliciani. Economic growth in the era of unconventional monetary instruments: A favar approach. Fournal of Macroeconomics, 2019.

[FO] Kenneth A Froot and Maurice Obstfeld. Intrinsic bubbles: The case of stock prices. Technical report.

[FO99] Gerald A Feltham and James A Ohlson. Residual earnings valuation with risk and stochastic interest rates. The Accounting Review, 74(2):165-183, 1999.

[GM02] Gustavo Grullon and Roni Michaely. Dividends, share repurchases, and the substitution hypothesis. The fournal of Finance, 57(4):1649-1684, 2002.

[GO04] Dan Gode and James Ohlson. Accounting-based valuation with changing interest rates. Review of Accounting Studies, 9(4):419-441, 2004.

[Goy12] Amit Goyal. Empirical cross-sectional asset pricing: a survey. Financial Markets and Portfolio Management, 26(1):3-38, 2012.

[Ham86] James D Hamilton. On testing for self-fulfilling speculative price bubbles. International Economic Review, pages 545-552, 1986.

[Han14] Lars Peter Hansen. Nobel lecture: Uncertainty outside and inside economic models. Fournal of Political Economy, 122(5):945-987, 2014.

[Hau01] Jerry Hausman. Mismeasured variables in econometric analysis: problems from the right and problems from the left. Journal of Economic Perspectives, 15(4):57-67, 2001.

[HB12] Ulrich Homm and Jörg Breitung. Testing for speculative bubbles in stock markets: a comparison of alternative methods. Fournal of Financial Econometrics, 10(1):198-231, 2012.

[HL98] John RM Hand and Wayne R Landsman. Testing the ohlson model: v or not v, that is the question. Available at SSRN 126308, 1998.

[HL05] John RM Hand and Wayne R Landsman. The pricing of dividends in equity valuation. Fournal of Business Finance \& Accounting, 32(3-4):435-469, 2005. 
[HO18] Yang Hu and Les Oxley. Bubbles in us regional house prices: evidence from house price-income ratios at the state level. Applied Economics, 50(29):31963229, 2018.

[JL05] Xiaoquan Jiang and Bon-Soo Lee. An empirical test of the accounting-based residual income model and the traditional dividend discount model. The fournal of Business, 78(4):1465-1504, 2005.

$\left[\mathrm{KK}^{+} 10\right]$ Gary Koop, Dimitris Korobilis, et al. Bayesian multivariate time series methods for empirical macroeconomics. Foundations and Trends ${ }^{\circledR}$ in Econometrics, 3(4):267-358, 2010.

[Lan10] Kevin J Lansing. Rational and near-rational bubbles without drift. The Economic fournal, 120(549):1149-1174, 2010.

[LCE13] Matthew R Lyle, Jeffrey L Callen, and Robert J Elliott. Dynamic risk, accounting-based valuation and firm fundamentals. Review of Accounting Studies, 18(4):899-929, 2013.

[Lin56] John Lintner. Distribution of incomes of corporations among dividends, retained earnings, and taxes. The American Economic Review, 46(2):97-113, 1956.

[LJ78] Robert E Lucas Jr. Asset prices in an exchange economy. Econometrica: Fournal of the Econometric Society, pages 1429-1445, 1978.

[LL01] Martin Lettau and Sydney Ludvigson. Consumption, aggregate wealth, and expected stock returns. the fournal of Finance, 56(3):815-849, 2001.

[LMS99] Charles MC Lee, James Myers, and Bhaskaran Swaminathan. What is the intrinsic value of the dow? The fournal of Finance, 54(5):1693-1741, 1999.

[LN09] Sydney C Ludvigson and Serena Ng. Macro factors in bond risk premia. The Review of Financial Studies, 22(12):5027-5067, 2009.

[LP81] Stephen F LeRoy and Richard D Porter. The present-value relation: Tests based on implied variance bounds. Econometrica: fournal of the Econometric Society, pages 555-574, 1981.

[LW04] Hedibert Freitas Lopes and Mike West. Bayesian model assessment in factor analysis. Statistica Sinica, 14(1):41-68, 2004. 
[MM61] Merton Miller and Franco Modigliani. Dividend policy, growth, and the valuation of shares. Journal of Business, pages 411-433, 1961.

[Mye99] James N Myers. Implementing residual income valuation with linear information dynamics. The Accounting Review, 74(1):1-28, 1999.

[NBW13] Ogonna Nneji, Chris Brooks, and Charles Ward. Intrinsic and rational speculative bubbles in the us housing market: 1960-2011. Fournal of Real Estate Research, 35(2):121-151, 2013.

[Ohl91] James A Ohlson. The theory of value and earnings, and an introduction to the ball-brown analysis. Contemporary Accounting Research, 8(1):1-19, 1991.

[Ohl95] James A Ohlson. Earnings, book values, and dividends in equity valuation. Contemporary accounting research, 11(2):661-687, 1995.

[OM93] Les Oxley and Michael McAleer. Econometric issues in macroeconomic models with generated regressors. Journal of Economic Surveys, 7(1):1-40, 1993.

[Ota02] Koji Ota. A test of the ohlson (1995) model: Empirical evidence from japan. The International fournal of Accounting, 37(2):157-182, 2002.

$\left[\mathrm{P}^{+} 17\right] \quad$ Alessia Paccagnini et al. Forecasting with FAVAR: macroeconomic versus financial factors. Economic Institute, 2017.

[Pag84] Adrian Pagan. Econometric issues in the analysis of regressions with generated regressors. International Economic Review, pages 221-247, 1984.

[Pen96] Stephen H Penman. The articulation of price-earnings ratios and market-tobook ratios and the evaluation of growth. Journal of Accounting Research, 34(2):235-259, 1996.

[PSY15] Peter CB Phillips, Shuping Shi, and Jun Yu. Testing for multiple bubbles: Historical episodes of exuberance and collapse in the s\&p 500. International Economic Review, 56(4):1043-1078, 2015.

[PWY11] Peter CB Phillips, Yangru Wu, and Jun Yu. Explosive behavior in the 1990s nasdaq: When did exuberance escalate asset values? International Economic Review, 52(1):201-226, 2011. 
[PY99] Daniel Peña and Victor Yohai. A fast procedure for outlier diagnostics in large regression problems. Fournal of the American Statistical Association, 94(446):434-445, 1999.

[Sen68] Pranab Kumar Sen. Estimates of the regression coefficient based on kendall's tau. Journal of the American Statistical Association, 63(324):1379-1389, 1968.

[Shi] Robert J Shiller. Do stock prices move too much to be justified by subsequent changes in dividends? Technical report.

[Shi81] Robert J. Shiller. Do stock prices move too much to be justified by subsequent changes in dividends? The American Economic Review, 71(3):421-436, 1981.

[SW98] James H Stock and Mark W Watson. Diffusion indexes. Technical report, National bureau of economic research, 1998.

[SW02] James H Stock and Mark W Watson. Forecasting using principal components from a large number of predictors. Journal of the American Statistical Association, 97(460):1167-1179, 2002.

[SW16] James H Stock and Mark W Watson. Dynamic factor models, factoraugmented vector autoregressions, and structural vector autoregressions in macroeconomics. In Handbook of macroeconomics, volume 2, pages 415-525. Elsevier, 2016.

[The50] H Theil. A rank-invariant method of linear and polynominal regression analysis (parts 1-3). In Ned. Akad. Wetensch. Proc. Ser. A, volume 53, pages 13971412, 1950.

[TLW08] Ruey S Tsay, Yi-Mien Lin, and Hsiao-Wen Wang. Residual income, valuerelevant information and equity valuation: a simultaneous equations approach. Review of Quantitative Finance and Accounting, 31(4):331-358, 2008.

[TLW09] Ruey S Tsay, Yi-Mien Lin, and Hsiao-Wen Wang. Residual income, nonearnings information, and information content. fournal of Forecasting, 28(6):487-511, 2009.

[W $\left.{ }^{+} 88\right]$ Kenneth D West et al. Dividend innovations and stock price volatility. Econometrica, 56(1):37-61, 1988. 
[WG08] Ivo Welch and Amit Goyal. A comprehensive look at the empirical performance of equity premium prediction. The Review of Financial Studies, 21(4):1455-1508, 2008.

[Whi19] Emily J Whitehouse. Explosive asset price bubble detection with unknown bubble length and initial condition. Oxford Bulletin of Economics and Statistics, 81(1):20-41, 2019.

[Yam18] Tomohiro Yamaguchi. The ohlson model and intangible assets: The problem of specifying'other information'. Available at SSRN 3142397, 2018.

[YKN15] Ky-Hyang Yuhn, Sang Bong Kim, and Joo Ha Nam. Bubbles and the weibull distribution: was there an explosive bubble in us stock prices before the global economic crisis? Applied Economics, 47(3):255-271, 2015.

[Yu11] Huayi Yu. Size and characteristic of housing bubbles in china's major cities: 1999-2010. China \& World Economy, 19(6):56-75, 2011. 
VITA

FAISAL AWWAL

June 23, 1987

2009

2012

2012-2015

2015-2016

2016-Present

2020-Present
Born, New York, New York

B.S.M, Finance

Tulane University

New Orleans, Louisiana

M.S., Financial Economics

University of New Orleans

New Orleans, Louisiana

Product Control Analyst

Barclays

New York, New York

Business Analyst

Credit Suisse

New York, New York

Adjunct Economics Instructor

Miami Dade College (Kendall Campus)

Miami, Florida

Senior Economist

Southeast Toyota Finance

Deerfield Beach, Florida 